\title{
Algorithmes et architectures pour ordinateurs quantiques supraconducteurs
}

\author{
par
}

\begin{abstract}
Alexandre Blais
Thèse présentée au département de physique en vue de l'obtention du grade de docteur ès sciences (Ph.D.)
\end{abstract}

FACUlté DES SCIENCES

UNIVERSITÉ DE SHERBROOKE

Sherbrooke, Québec, Canada, décembre 2002 


\title{
Le $\frac{6 \text { déémpre } 2002}{\text { date }}$
}

le jury a accepté la thèse de M. Alexandre Blais dans sa version finale.

\section{Composition du jury}

\author{
Membre : $\quad$ M. André-Marie Tremblay \\ Département de physique \\ Membre : $\quad$ M. Serge Lacelle \\ Département de chimie \\ Membre : $\quad$ Mme Karyn Le Hur \\ Département de physique \\ Membre externe : $\quad$ M. Michel Devoret \\ University Yale \\ Membre et \\ président-rapporteur : Patrick Fournier \\ Département de physique
}




\section{Sommaire}

Depuis sa formulation, la théorie de l'information a été basée, implicitement, sur les lois de la physique classique. Une telle formulation est toutefois incomplète puisqu'elle ne tient pas compte de la réalité quantique. Au cours des vingt dernières années, l'expansion de la théorie de l'information englobant les effets purement quantiques a connu un intérêt grandissant. La réalisation d'un système de traitement de l'information quantique, un ordinateur quantique, présente toutefois de nombreux défis. Dans ce document, on s'intéresse à différents aspects concernant ces défis. On commence par présenter des concepts algorithmiques comme l'optimisation de calculs quantiques et le calcul quantique géométrique. Par la suite, on s'intéresse au design et à différents aspects de l'utilisation de qubits basés sur les jonctions Josephson. En particulier, un nouveau design de qubit supraconducteur est suggéré. On présente aussi une approche originale pour l'interaction entre qubits. Cette approche est très générale puisqu'elle peut être appliquée à différents designs de qubits. Finalement, on s'intéresse à la lecture des qubits supraconducteurs de flux. Le détectẹur suggéré ici a l'avantage de pouvoir être découplé du qubit lorsqu'il n'y a pas de mesure en cours. 


\section{Remerciements}

Je tiens d'abord à exprimer toute ma gratitude à mes directeurs de recherche André-Marie Tremblay et Serge Lacelle. Ceux-ci m'ont donné toute liberté pour explorer différentes avenues. Grâce à des discussions intéressantes, certaines de ces avenues ont abouti aux résultats présentés dans ce document. Les 'qubits supraconducteurs' ne font pas partie de leurs intérêts de recherche immédiats et je leur en remercie d'autant plus de m'avoir soutenu durant toutes ces années.

Je souhaite vivement remercier les chercheurs et employés de D-Wave Systems, Inc. et, plus particulièrement, Alexandre Zagoskin pour m'avoir donné l'opportunité de travailler avec eux, pour leur confiance et les nombreux contacts qu'ils ont rendus possibles. Les trois dernières années auraient été beaucoup plus difficiles s'ils n'avaient pas été disponibles pour discuter et échanger des idées. Je les remercie aussi pour le bon temps passé en leur compagnie à Vancouver : le travail comme les soupers avec. un bon vin!

$\mathrm{Au}$ cours de la réalisation des différents projets présentés ici, j'ai grandement bénéficié de discussions avec Martin Beaudry, Daniel Lidar, John Martinis et Alexandre Shnirman. Je les remercie chaleureusement.

J'exprime aussi ma reconnaissance à Michel Devoret, Patrick Fournier et Karyn LeHur d'avoir accepté d'être membre du jury de thèse.

De plus, les étudiants et le personnel du département de physique ont contribué grandement à rendre ces dernières années agréables au quotidien et je leur en suis reconnaissant.

Finalement, sans le soutien constant d'Hélène ce travail n'aurait certainement pas abouti à ce qui est présenté ici. Je la remercie pour sa patience au cours des longues semaines (années?) de travail, pour sa compréhension à chacune de mes nombreuses absences et pour avoir partagé avec moi l'enthousiasme que j'ai pour ce travail.

Je remercie le CRSNG, D-Wave Systems, Inc., le fond FCAR et l'IMSI pour leur support financier. 


\section{Table des matières}

$\begin{array}{ll}\text { Sommaire } & \text { iii }\end{array}$

Remerciements iv

Table des matières $v$

Table des figures $\quad$ vii

$\begin{array}{ll}\text { Introduction } & 1\end{array}$

1 Calcul quantique 4

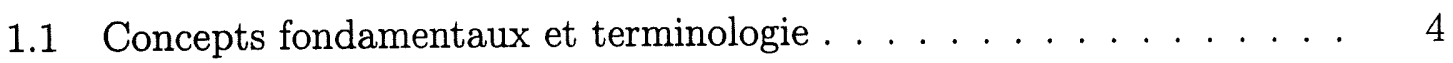

1.2 Le paradigme standard . . . . . . . . . . . . . . . . 7

1.3 Opérations logiques et universalité $\ldots \ldots \ldots \ldots \ldots \ldots$

1.4 Hamiltoniens et opérations logiques . . . . . . . . . . . . . . 11

1.5 Qubits encodés . . . . . . . . . . . . . . . . . . 13

1.5.1 Corrections quantiques d'erreurs . . . . . . . . . . . . 13

1.5.2 Sous-espaces sans décohérence . . . . . . . . . . . . 16

1.5.3 Universalité encodée . . . . . . . . . . . . . . . 17

1.6 Algorithmes quantiques . . . . . . . . . . . . . . . . . . . 21

1.6.1 Transformée de Fourier quantique . . . . . . . . . . . . 22

1.6.2 Algorithme d'estimation de phase . . . . . . . . . . . 23

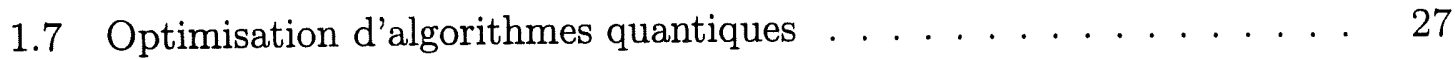

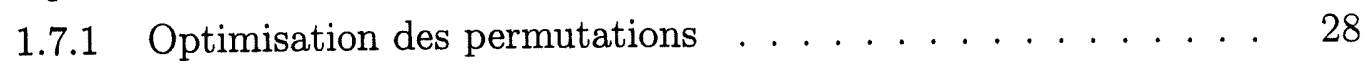

1.7 .2 Circuits optimisés . . . . . . . . . . . . . 30 
1.7.3 Optimisation par recuit simulé . . . . . . . . . . . . 33

1.7.4 Remarques et sommaire . . . . . . . . . . . . . . . 34

2 Calcul quantique géométrique 36

2.1 Portes géométriques adiabatiques vs non-adiabatiques . . . . . . . . . 38

2.1.1 Phase de Berry et calcul géométrique . . . . . . . . . . . . 38

2.1.2 Phase géométrique non-adiabatique . . . . . . . . . . . . 41

2.1.3 Calcul géométrique non-adiabatique . . . . . . . . . . . . 43

2.2 Tolérance au bruit des paramètres de contrôle . . . . . . . . . . . . . 46

2.3 Géométrique $v s$ topologique . . . . . . . . . . . . . . . . . . . . . . . 54

2.4 Sommaire . . . . . . . . . . . . . . . . . . . 55

3 Qubits supraconducteurs $\quad 57$

3.1 Circuits électriques quantiques . . . . . . . . . . . . . . . 58

3.1.1 Exemple additionnel : la boîte de Cooper . . . . . . . . . . 63

3.2 Réduction vers un système à deux niveaux . . . . . . . . . . . 65

3.2.1 Qubit de Charge ................ 65

3.2 .2 Qubit de phase ................ 68

3.3 Relaxation et déphasage . . . . . . . . . . . . . . 70

3.4 Qubits supraconducteurs : designs et réalisations . . . . . . . . . . 75

3.4.1 Qubits basés sur la charge . . . . . . . . . . . . . 75

3.4.2 Qubits basés sur la phase . . . . . . . . . . . . 77

3.4.3 Designs récents . . . . . . . . . . . . . . . 80

3.5 Sommaire . . . . . . . . . . . . . . . . . . . 84

4 Interaction qubit-qubit ajustable $\quad 85$

4.1 Méthodes d'interaction pour qubits supraconducteurs . . . . . . . . 85

4.1 Qubits de charge ................ 85

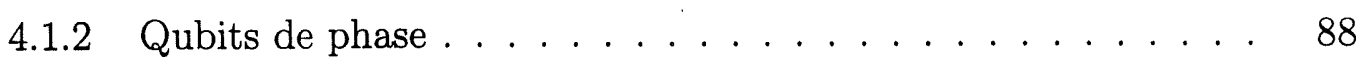

4.1 .3 Designs récents . . . . . . . . . . . . . . . . 90

4.2 Interaction résonante entre CBJJ . . . . . . . . . . . . . . . 90

4.2.1 Déphasage et relaxation du circuit résonant . . . . . . . . . . 97

4.3 Interaction résonante pour qubits supraconducteurs . . . . . . . . . . 101 
4.3.1 Interaction qubit-qubit universelle . . . . . . . . . . 105

4.3.2 Approximation séculaire . . . . . . . . . . . . . 106

4.3.3 Déphasage et relaxation . . . . . . . . . . . . . . 108

4.4 Sommaire . . . . . . . . . . . . . . . . . . . . . . . . 112

5 Lecture d'un qubit de phase supraconducteur $\quad 116$

5.1 Aspects de la mesure en mécanique quantique . . . . . . . . . . . . . 117

5.2 Amplificateurs linéaires . . . . . . . . . . . . . . . . . . . . . 120

5.3 Détecteurs pour qubits supraconducteurs . . . . . . . . . . . . . . . 121

5.3.1 Lecture du qubit de phase par un SQUID-dc . . . . . . . . . . 121

5.3 .2 Autres stratégies . . . . . . . . . . . . . . . . . . . 124

5.4 Lecture du qubit de flux . . . . . . . . . . . . . . . . . 126

5.4.1 Contrôle des fluctuations phase-charge dans un SSET . . . . . 127

5.4.2 Transducteur flux-charge pour qubits de flux . . . . . . . . 128

5.4 .3 Sensibilité et rétroaction . . . . . . . . . . . . . . . 137

5.4 .4 Remarques et sommaire . . . . . . . . . . . . . . . . . . 141

$\begin{array}{ll}\text { Conclusion } & 143\end{array}$

A Phase géométrique sur qubit de charge 146

A.1 Phase géométrique adiabatique . . . . . . . . . . . . . . 146

A.2 Phase géométrique non-adiabatique . . . . . . . . . . . . . 149

B Distance pour opérations dynamiques bruyantes 151

C Encodage et suppression d'erreurs pour qubits de phase supraconducteurs

$\begin{array}{ll}\text { D Qubits à terminaux multiples } & 183\end{array}$

E Taux de déphasage et de relaxation $\quad 189$

$\begin{array}{lc}\text { Bibliographie } & 193\end{array}$ 


\section{Table des figures}

1.1 La sphère de Bloch. . . . . . . . . . . . . . . . . . 5

1.2 Circuits quantiques. . . . . . . . . . . . . . . . 10

1.3 Circuit quantique détectant le renversement d'un bit. . . . . . . . . 14

1.4 Universalité encodée. . . . . . . . . . . . . . . . . . . . . . . . 19

1.5 Circuit quantique pour la QFT. . . . . . . . . . . . . 23

1.6 Circuit quantique pour l'estimation de phase. . . . . . . . . . . . 24

1.7 Opération contrôlée sur des qubits distants. $\ldots \ldots \ldots \ldots$

1.8 Circuit quantique optimisé pour $\mathrm{F}_{5} \ldots \ldots \ldots \ldots \ldots \ldots \ldots \ldots$

1.9 Comparaison du coût en temps pour la QFT. . . . . . . . . . . . 32

1.10 Circuit amélioré par recuit simulé pour la QFT sur cinq qubits. . . . 34

2.1 Sphère de Bloch et espace projectif. . . . . . . . . . . . . . 42

2.2 Évolution du vecteur de Bloch sur la sphère de Bloch sous la séquence

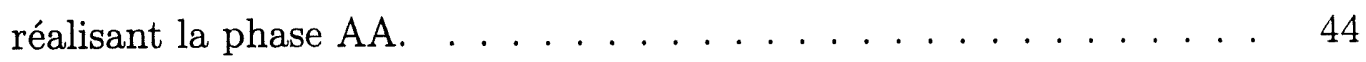

2.3 Distance en fonction de $\theta$ et de l'amplitude maximale du bruit. . . . . 50

2.4 Parcours dans l'espace des paramètres réalisant la porte de phase adia-

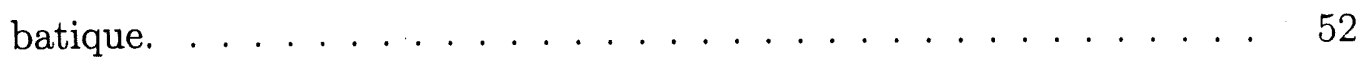

3.1 Convention de signes pour les voltages et courants de branche. . . . 58

3.2 Exemple de circuit électrique : boucle supraconductrice formée de trois jonctions Josephson. . . . . . . . . . . . . . 60

3.3 Boîte de Cooper. . . . . . . . . . . . . . . . . . 63

3.4 Énergie de la boîte de Cooper. . . . . . . . . . . . . . . 66

3.5 Design modifié pour le qubit de phase à trois jonctions. . . . . . . . 68 
3.6 Courbes de niveaux de l'énergie potentielle pour le qubit de phase à

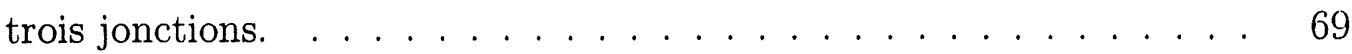

3.7 Modélisation des sources de bruit pour les qubits supraconducteurs. . $\quad 72$

3.8 Modèle de Caldeira-Leggett pour une impédance et une admittance. . 73

3.9 Design utilisé pour l'expérience de Nakamura et al. . . . . . . . . 76

3.10 Qubits basés sur les supraconducteurs de type $d \ldots \ldots \ldots \ldots \ldots$

3.11 Qubit phase-charge de Saclay. . . . . . . . . . . . . . . . . 81

3.12 Qubit basé sur une jonction unique. . . . . . . . . . . . . 83

4.1 Qubits couplés en parallèle à une inductance $L . \ldots \ldots \ldots \ldots$. . . . 87

4.2 Paire de qubits CBJJ couplés par une capacité $C_{c} \ldots \ldots \ldots \ldots 1$

4.3 Potentiel cubique associé à une CBJJ. . . . . . . . . . . . . . . 92

4.4 Représentation des états logiques et des états propres sur la sphère de Bloch. . . . . . . . . . . . . . . . . . . . . . . 98 98

4.5 Interaction entre qubits supraconducteurs. . . . . . . . . . . . . 101

4.6 Séquence d'interactions qubits-jonction pour la réalisation de l'opération $\sqrt{\mathrm{SWAP}} \ldots \ldots \ldots \ldots \ldots \ldots \ldots$

4.7 Temps de relaxation et déphasage en présence de la CBJJ _ . . . . 110

4.8 Design intégré pour l'interaction et la mesure de qubits supraconducteurs.114

5.1 Qubit de phase couplé inductivement à un SQUID-dc. . . . . . . . . . 122

5.2 Courbe IV pour une jonction hystérétique. . . . . . . . . . . . . . 123

5.3 Mesure d'un qubit de charge par un SSET. . . . . . . . . . . . . 125

5.4 Circuit utilisé par Matters et al. pour étudier l'effet des fluctuations de phase dans un système dans le régime de blocage de Coulomb. . . . . 127

5.5 Résultats expérimentaux de Matters et al. . . . . . . . . . . . . 129

5.6 Nouveau design pour la lecture d'un qubit de flux. . . . . . . . . . . 130

5.7 Courant critique dans le système SSET-SQUID-qubit obtenu dans l'approximation à deux charges. . . . . . . . . . . . . . . . . . . 133

5.8 Courant critique dans le système SSET-SQUID-qubit obtenu par diagonalisation exacte. . . . . . . . . . . . . . . . 135

5.9 Courant critique dans le système SSET-SQUID-qubit obtenu par diagonalisation exacte. . . . . . . . . . . . . . . . 136 
A.1 Circuit dans l'espace des paramètres $\left\{\Phi_{x}, n_{g}\right\} \ldots \ldots \ldots \ldots$

A.2 Phase de Berry en fonction $n_{g m}$ et $\Phi_{x m} \ldots \ldots \ldots \ldots \ldots \ldots$

A.3 Séquence de flux externe $\Phi_{x}$ et de charge de grille $n_{g}$ réalisant l'opération $R_{z}^{A A}(\theta) \ldots \ldots \ldots \ldots \ldots \ldots \ldots \ldots \ldots$

A.4 Phase géométrique, champ effectif $B_{n}$ et temps d'opération en fonction des paramètres de contrôle. . . . . . . . . . . . . . . 150 


\section{Introduction}

L'informatique quantique est un domaine en émergence faisant appel à plusieurs

spécialités : physique, génie, chimie, informatique et mathématiques. L'objectif visé par cette intégration de connaissances est la réalisation d'un ordinateur quantique et l'utilisation d'un tel ordinateur pour effectuer certains calculs beaucoup plus rapidement qu'avec un ordinateur fonctionnant de façon standard (ordinateur classique). Cette accélération est rendue possible en tirant profit des phénomènes quantiques tels que les superpositions d'états, l'enchevêtrement et l'interférence [1].

Quel que soit l'angle disciplinaire sous lequel on l'envisage, les défis dans ce domaine sont de taille. Par exemple, très peu d'algorithmes quantiques (algorithmes adaptés pour fonctionner sur un ordinateur quantique) efficaces sont connus. De même, plusieurs aspects concernant l'enchevêtrement ne sont pas encore bien compris. Ces concepts ne pourront toutefois être applicables qu'une fois un ordinateur quantique dûment réalisé. À ce jour, un prototype d'ordinateur quantique à sept bits quantiques (qubits) a pu être réalisé expérimentalement en utilisant les techniques de résonance magnétique nucléaire [2]. Malheureusement, on sait que cette technique ne pourra pas permettre la réalisation d'un ordinateur de taille beaucoup plus importante [3].

De ce point de vue, les qubits basés sur les systèmes de l'état solide et sur l'utilisation des techniques standards de lithographie ont plus de chance de permettre la réalisation d'un ordinateur quantique de taille utile. Pour ces systèmes toutefois, maintenir la cohérence de phase sur des échelles de temps suffisamment longues pour réaliser des calculs utiles est un problème important. En ce sens, les matériaux supraconducteurs semblent être avantageux puisqu'il est possible de tirer profit de la cohérence quantique macroscopique de l'état supraconducteur. De même, l'étude du 
comportement quantique de ces systèmes mésoscopiques présente un intérêt fondamental.

Ce document a pour objet principal l'étude des ordinateurs quantiques basés sur les systèmes supraconducteurs et, plus particulièrement, les jonctions Josephson. Dans le premier chapitre, on présente certains concepts fondamentaux reliés à l'informatique quantique : qubit, enchevêtrement, opérations logiques, corrections d'erreurs et algorithmes quantiques. On décrit aussi une approche originale concernant l'optimisation des algorithmes quantiques.

Le chapitre 2 porte sur l'application des phases géométriques au calcul quantique. La phase géométrique adiabatique (phase de Berry) a récemment reçu beaucoup d'attention puisqu'elle semble permettre de réaliser les opérations logiques en minimisant l'effet des imperfections. On explique ici comment utiliser' la phase géométrique nonadiabatique dans le même but et comment cette phase peut être observée à l'aide de qubits supraconducteurs de charge. Une étude plus approfondie de la sensibilité aux fluctuations montre toutefois que ces phases ne sont pas avantageuses pour le calcul quantique.

C'est au chapitre 3, que les qubits supraconducteurs sont introduits. On présente alors différents designs et certains résultats expérimentaux récents. Une partie de notre contribution au domaine des qubits supraconducteurs a été présentée dans un autre contexte et ne sera pas répétée ici $[4,5]$. De plus, afin de ne pas alourdir ce document, certaines contributions plus récentes ne sont que brièvement abordées dans le texte. Le lecteur intéressé trouvera plus de détails aux annexes $\mathrm{C}$ et $\mathrm{D}$ où les publications décrivant ces résultats ont été reproduites.

Dans le but de mener à terme un calcul quantique, il sera nécessaire de réaliser des opérations logiques faisant intervenir plus d'un qubit. De même, vu l'état des réalisations expérimentales sur les qubits supraconducteurs isolés [6-8], le prochain défi expérimental semble être l'enchevêtrement d'une paire de ces qubits. Motivé par ces questions algorithmiques et expérimentales, on s'intéresse au chapitre 4 à l'interaction entre qubits supraconducteurs. On suggère une approche pouvant, en principe, être utilisée avec différents types de qubits.

Finalement, le chapitre 5 aborde la question de la mesure. Afin d'extraire les résultats du calcul, il sera nécessaire de mesurer l'état de l'ordinateur quantique. Le 
design d'un appareil de mesure n'est pas simple car celui-ci ne doit pas affecter les qubits lors des manipulations cohérentes mais doit être capable de lire rapidement et efficacement le qubit au moment voulu. On présente dans ce chapitre certains résultats sur un design original d'appareil de mesure.

Puisque l'informatique quantique et les qubits supraconducteurs ne sont pas des axes principaux de recherche à l'Université de Sherbrooke, on a ici opté pour un style, on l'espère, pédagogique. De même, afin de situer le lecteur, chacun des chapitres comporte une courte revue de la littérature pertinente. 


\section{Chapitre 1}

\section{Calcul quantique}

So I commend quantum computation to those young philosophers and old physicists who have the time and inclination to think about these issues. It's by far the most interesting game currently in town.

-N. David Mermin

\subsection{Concepts fondamentaux et terminologie}

L'unité fondamentale de l'information quantique est le bit quantique ou, plus simplement qubit (de l'anglais 'quantum bit'). Ce terme a été introduit par B. Shumacher [9]. Comme sa contrepartie classique, un qubit peut prendre deux valeurs dénotées $|0\rangle$ et $|1\rangle$. Un qubit vit donc dans un espace d'Hilbert à deux dimensions et peut être dans une superposition de ces deux états orthogonaux

$$
a|0\rangle+b|1\rangle
$$

La base $\{|0\rangle,|1\rangle\}$ est parfois appelée base de calcul. Il est intéressant de mentionner que, selon le théorème de Holevo [10], seulement un bit classique peut être extrait d'un bit quantique.

Lorsque l'on s'intéresse à un seul qubit, le vecteur de Bloch $\boldsymbol{b}(t)$ sur la sphère de Bloch est une représentation utile. Ce vecteur est défini à partir de l'opérateur de 


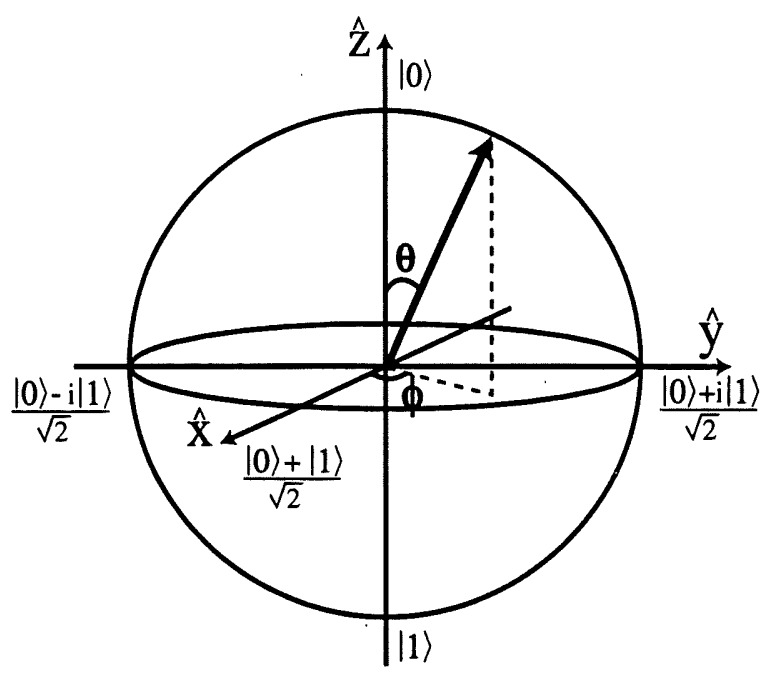

Fig. 1.1: La sphère de Bloch est utile pour représenter l'état d'un système à deux niveaux. Le pôle nord correspond à l'état $|0\rangle$ tandis que le pôle sud correspond à $|1\rangle$.

densité de la façon suivante :

$$
\rho(t)=|\psi(t)\rangle\langle\psi(t)|=\frac{1}{2}(\mathbb{I}+\boldsymbol{b}(t) \cdot \boldsymbol{\sigma}),
$$

où $\sigma$ est le vecteur des matrices de Pauli et $\mathbb{I}$ la matrice identité. Pour les états purs, $\operatorname{Tr}\left(\rho^{2}\right)=1$ et $\boldsymbol{b}(t)$ est un vecteur unité. L'utilité de cette représentation vient du fait que l'état le plus général à un qubit peut être écrit sous la forme

$$
\cos (\theta / 2)|0\rangle+\sin (\theta / 2) e^{i \phi}|1\rangle
$$

où les 'angles' $\theta$ et $\phi$ peuvent être considérés comme les coordonnées sur la sphère de Bloch. La Figure 1.1 présente cette construction.

On nommera un ensemble de $n$ qubits un registre quantique. Afin de simplifier la notation, on utilisera parfois la représentation décimale plutôt que d'écrire explicitement la chaîne binaire correspondant aux $n$ qubits. Dans cette notation, une superposition arbitraire de $n$ qubits s'écrit

$$
\sum_{x=0}^{2^{n}-1} c_{x}|x\rangle
$$

où les amplitudes $c_{x}$ satisfont la règle de normalisation habituelle $\sum_{x=0}^{2^{n}-1}\left|c_{x}\right|^{2}=1$. 


\subsection{CONCEPTS FONDAMENTAUX ET TERMINOLOGIE}

Une propriété importante des qubits est qu'ils peuvent être enchevêtrés (entangled, parfois traduit par intriqués) avec d'autres qubits. Deux systèmes quantiques sont dits enchevêtrés si l'état du système total ne peut s'écrire comme un produit tensoriel de ses parties. Dans le contexte du calcul quantique, les quatre états de Bell sont des états enchevêtrés particulièrement utiles :

$$
\begin{aligned}
& \left|\phi_{ \pm}\right\rangle=\frac{1}{\sqrt{2}}(|00\rangle \pm|11\rangle), \\
& \left|\psi_{ \pm}\right\rangle=\frac{1}{\sqrt{2}}(|01\rangle \pm|10\rangle) .
\end{aligned}
$$

Leur utilité provient du fait que ce sont des états d'enchevêtrement maximal. Dans un espace d'Hilbert de dimensions $2 \times 2$, les états d'enchevêtrement maximal sont tels que la matrice densité réduite, obtenue en faisant la trace sur l'un ou l'autre des sous-systèmes, représente un état complètement mélangé :

$$
\rho_{1(2)}=\operatorname{Tr}_{2(1)} \rho_{12}=\frac{1}{2} \mathbb{I} \text {. }
$$

En d'autres mots, pour les états d'enchevêtrement maximal, toute l'information est contenue dans les corrélations entre les sous-systèmes. Les sous-systèmes, individuellement, ne contiennent donc pas d'information.

Différentes mesures ont été suggérées dans la littérature pour quantifier la 'quantité' d'enchevêtrement d'un état donné. Pour les états purs, l'entropie de Von Newman $S(\rho)=-\operatorname{Tr}(\rho \log \rho)$ constitue une mesure unique [11]. Toutefois, pour un état mixte arbitraire, une telle mesure n'est pas connue. Il est intéressant de mentionner que pour les états mixtes à deux qubits, le critère de la transposée partielle positive peut être utilisé pour déterminer si un état est enchevêtré ou non (ce critère fonctionne aussi pour les systèmes $2 \times 3$, soit un qubit et un qutrit.) [12]. De même, toujours pour les états mixtes à deux qubits, la concurrence [13], où de façon équivalente l'enchevêtrement de formation [14], peut quantifier la quantité d'enchevêtrements. Pour les espaces d'Hilbert de dimensions supérieures, il n'existe pas, à ce jour, de mesure unique.

L'enchevêtrement semble être responsable de la puissance de calcul des ordinateurs quantiques [15]. Cette question fait toutefois l'objet d'un débat, particulièrement dans le contexte du calcul quantique en RMN de l'état liquide $[16,17]$. Le lecteur intéressé pourra consulter la référence [18] pour un tour d'horizon des différents points de vue. 


\subsection{Le paradigme standard}

Le 'paradigme standard' du calcul quantique a été énoncé clairement pour la première fois par D. DiVincenzo et constitue ce que l'on nomme maintenant les 'critères de DiVincenzo' [19]. Ce sont les cinq critères de base qu'un ordinateur quantique doit satisfaire :

1. Qubits : L'espace d'Hilbert doit pouvoir être partitionné en un produit de $n$ systèmes à deux niveaux. En d'autres mots, il doit y avoir des qubits.

2. Initialisation : Il doit être possible d'initialiser le registre quantique dans un état connu, souvent choisi comme $|0\rangle=|000 \cdots 000\rangle$.

3. Calcul : Il doit être possible d'appliquer un ensemble universel de portes logiques sur le registre (plus de détails sur l'universalité plus bas).

4. Cohérence : L'interaction entre les qubits et leur(s) environnement(s) doit être suffisamment faible de sorte qu'il soit possible de maintenir la cohérence tout au long du calcul.

5. Mesure : Chaque qubit doit pouvoir subir une mesure projective et la valeur du bit classique qui en résulte doit être obtenue avec une grande efficacité.

Depuis leur publication, il a été montré que ces critères peuvent être assouplis [20]. Certains aspects de ces développements plus récents seront discutés à la section $\S 1.5$ et, pour le moment, concentrons-nous sur ce paradigme standard.

Il est évident que de satisfaire ces conditions dans un seul système physique est une tâche difficile. Par exemple, le critère \#4 stipule que la cohérence doit être maintenue pendant tout le calcul. Ceci est nécessaire afin de tirer profit de l'accélération quantique des calculs due aux superpositions d'états et à l'enchevêtrement. En pratique, le nombre de qubits nécessaires pour effectuer des calculs utiles sera important. Ces états à $n$ qubits interagiront avec le grand nombre de degrés de liberté de leur environnement, ce qui provoque la perte de cohérence dans le système. Il s'agit du phénomène de décoherence [21].

D'autre part, le critère \#3 stipule qu'il doit être possible de manipuler le système quantique et par conséquent, qu'il est possible d'interagir avec celui-ci à partir du 'monde extérieur'. Cette connexion entre le monde extérieur hostile (puisque classique) et le système quantique fragile est une bonne façon d'ouvrir la porte à la 
décohérence. Ce qui est encore pire est que les conditions \#2 et \#5 impliquent que l'environnement peut respectivement relaxer et déphaser rapidement les qubits. Plus de détails seront donnés sur ces processus aux prochains chapitres. Ainsi, les critères qu'un ordinateur quantique doit satisfaire sont difficiles à réaliser et, jusqu'à un certain point, sont en contradiction les uns avec les autres.

L'initialisation et la mesure seront discutées en plus de détails dans les chapitres subséquents. Concentrons-nous ici sur la manipulation de l'information quantique.

\subsection{Opérations logiques et universalité}

Pour l'information classique, la possibilité de réaliser les portes logiques NOT et NAND sur un ensemble de bits classiques permet de réaliser n'importe quel calcul classique. On dit donc que \{NOT, NAND \} forment un ensemble universel (complet) pour le calcul classique [22].

Pour le calcul quantique, un ensemble universel doit aussi être défini. Avant de présenter un tel ensemble, il est toutefois nécessaire de bien comprendre ce qu'est un calcul quantique. Dans le paradigme standard, ce n'est rien de plus que l'évolution unitaire contrôlée d'un syst ne quantique

$$
|\psi(t)\rangle=\mathrm{U}(t)|\psi(0)\rangle
$$

où $|\psi(0)\rangle$ est l'état initial de l'ordinateur (habituellement choisi comme $|0\rangle=$ $|000 \cdots 000\rangle)$ et $|\psi(t)\rangle$ l'état final qui, une fois mesuré, sera la réponse au calcul. L'opérateur unitaire d'évolution $\mathrm{U}(t)$ représente le programme quantique : la dynamique du système est choisie de façon à correspondre au calcul à effectuer. En termes plus physiques, l'hamiltonien responsable de l'évolution est manipulé de façon à générer le calcul quantique voulu.

Puisqu'une opération logique quantique est un opérateur d'évolution unitaire, alors un ensemble universel de portes logiques quantiques devrait pouvoir réaliser $U\left(2^{n}\right)$ sur $n$ qubits. Ici, $U\left(2^{n}\right)$ est le groupe des matrices unitaires de dimensions $2^{n} \times 2^{n}$. Évidemment, puisqu'une phase globale est sans conséquence physique, nous serons satisfaits avec $S U\left(2^{n}\right)$ qui est le groupe des matrices unitaires spéciales dont les membres ont un déterminant égal à l'unité. 


\subsection{OPÉRATIONS LOGIQUES ET UNIVERSALITÉ}

Comme dans le cas classique, il existe plusieurs ensembles universels. Un ensemble particulièrement utile est formé par une porte non-triviale à deux qubits et toutes les portes à un qubit [23]. Dans le présent contexte, non-triviale signifie que la porte peut créer de l'enchevêtrement. De même, il découle de la discussion précédente que l'ensemble des portes à un qubit correspond à $S U(2)$ dont les générateurs sont les matrices de Pauli (dans la base $\{|0\rangle,|1\rangle\}$ )

$$
\sigma_{x}=\left(\begin{array}{cc}
0 & 1 \\
1 & 0
\end{array}\right) ; \quad \sigma_{y}=\left(\begin{array}{cc}
0 & -i \\
i & 0
\end{array}\right) ; \quad \sigma_{z}=\left(\begin{array}{cc}
1 & 0 \\
0 & -1
\end{array}\right) .
$$

Les opérations correspondantes sont des rotations à un qubit sur la sphère de Bloch

$$
\mathrm{R}_{\alpha}(\theta)=e^{-i \theta \sigma_{\alpha} / 2}
$$

avec $\alpha=x, y, z$. Évidemment, pour couvrir toute la sphère de Bloch, des rotations selon deux axes orthogonaux suffisent. Ainsi, seuls deux des générateurs de $S U(2)$ seront nécessaires en pratique.

Pour l'opération à deux qubits, la seule contrainte est qu'elle permette de créer de l'enchevêtrement. Une opération standard ayant cette capacité est le non-contrôlé (controlled-NOT), noté $\mathrm{C}_{\mathrm{NOT}}$. Cette porte a pour action d'inverser la valeur du second bit (bit cible) si le premier (bit source) est dans l'état $|1\rangle$ :

$$
C_{\text {NOT }}:\left\{\begin{array} { l } 
{ | 0 0 \rangle } \\
{ | 0 1 \rangle } \\
{ | 1 0 \rangle } \\
{ | 1 1 \rangle }
\end{array} \mapsto \left\{\begin{array}{l}
|00\rangle \\
|01\rangle \\
|11\rangle \\
|10\rangle
\end{array}\right.\right.
$$

Dans la base de calcul, cette opération a donc la forme suivante :

$$
C_{\text {NOT }}=\left(\begin{array}{llll}
1 & & & \\
& 1 & & \\
& & 0 & 1 \\
& & 1 & 0
\end{array}\right) .
$$

Toutes opérations à deux qubits équivalentes peuvent évidemment prendre la place $\mathrm{du} \mathrm{C}_{\text {NOT }}$ pour compléter l'ensemble universel. Une porte $W$ est dite équivalente à une 


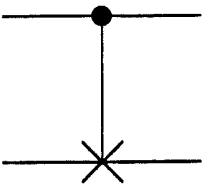

(a)

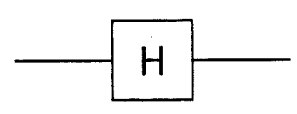

(b)

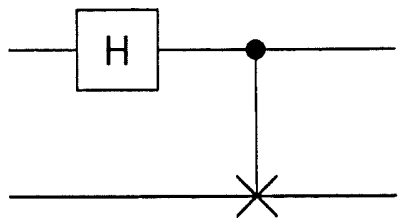

(c)

FIG. 1.2: a) Circuit quantique représentant la porte $C_{\text {NOT }}$. La ligne du haut représente le bit de contrôle et celle du bas, le bit cible : |contrôle, cible $\rangle$. Le temps s'écoule de la gauche vers la droite. b) Porte d'Hadamard. c) Circuit quantique passant de la base de calcul à la base des états de Bell.

porte $\mathrm{V}$ si $\mathrm{W}$ peut être obtenue à partir du produit d'un nombre fini d'opérations à un qubit et de $V$. Une procédure constructive et presque optimale pour obtenir le non-contrôlé à partir d'une porte équivalente existe [24].

La représentation du $C_{\text {NOT }}$ sous la forme de circuit quantique est présentée à la figure $1.2 \mathrm{a}$ ). Dans cette notation, chaque ligne horizontale représente un qubit et le temps s'écoule de la gauche vers la droite.

Une porte à un qubit souvent utilisée est la transformation de Hadamard $H$. Dans la base de calcul, cette opération a l'action et la représentation matricielle suivante :

$$
H:\left\{\begin{array} { l } 
{ | 0 \rangle } \\
{ | 1 \rangle }
\end{array} \mapsto \frac { 1 } { \sqrt { 2 } } \left\{\begin{array}{l}
|0\rangle+|1\rangle \\
|0\rangle-|1\rangle
\end{array} \quad \mathrm{H}=\frac{1}{\sqrt{2}}\left(\begin{array}{cc}
1 & 1 \\
1 & -1
\end{array}\right) .\right.\right.
$$

Le circuit quantique représentant cette porte se trouve à la figure $1.2 \mathrm{~b}$ ).

On combine les opérations logiques pour former des circuits plus complexes. Considérons par exemple le circuit quantique de la figure Figure $1.2 \mathrm{c}$ ). Son action sur les quatre états de la base de calcul est simplement :

$$
\begin{aligned}
& |00\rangle \stackrel{\mathrm{H}}{\longrightarrow} \frac{1}{\sqrt{2}}(|0\rangle+|1\rangle)|0\rangle \stackrel{\mathrm{C}_{\mathrm{NOT}}}{\longrightarrow} \frac{1}{\sqrt{2}}(|00\rangle+|11\rangle)=\left|\phi^{+}\right\rangle ; \\
& |01\rangle \stackrel{\mathrm{H}}{\longrightarrow} \frac{1}{\sqrt{2}}(|0\rangle+|1\rangle)|1\rangle \stackrel{\mathrm{C}_{\mathrm{NOT}}}{\longrightarrow} \frac{1}{\sqrt{2}}(|01\rangle+|10\rangle)=\left|\psi^{+}\right\rangle ; \\
& |10\rangle \stackrel{\mathrm{H}}{\longrightarrow} \frac{1}{\sqrt{2}}(|0\rangle-|1\rangle)|0\rangle \stackrel{\mathrm{C}_{\mathrm{NOT}}}{\longrightarrow} \frac{1}{\sqrt{2}}(|00\rangle-|11\rangle)=\left|\phi^{-}\right\rangle ; \\
& |11\rangle \stackrel{\mathrm{H}}{\longrightarrow} \frac{1}{\sqrt{2}}(|0\rangle-|1\rangle)|1\rangle \stackrel{\mathrm{C}_{\mathrm{NOT}}}{\longrightarrow} \frac{1}{\sqrt{2}}(|01\rangle-|10\rangle)=\left|\psi^{-}\right\rangle .
\end{aligned}
$$


Ce circuit quantique fait donc le passage entre la base de calcul et la base des états de Bell (1.5) en enchevêtrant les états initiaux séparables.

Un autre ensemble complet de portes logiques quantiques utile est $\left\{H, T, C_{\text {NOT }}\right\}$ [25], où

$$
\mathbf{T}=\left(\begin{array}{ll}
1 & \\
& e^{i \pi / 4}
\end{array}\right)=e^{i \pi / 8}\left(\begin{array}{ll}
e^{-i \pi / 8} & \\
& e^{i \pi / 8}
\end{array}\right) .
$$

Cette opération est parfois aussi appelée 'porte $\pi / 8$ '. Contrairement à l'ensemble présenté plus haut, celui-ci n'est formé que de portes discrètes et il ne peut être utilisé pour réaliser exactement un opérateur unitaire arbitraire. Lorsque cet ensemble est utilisé, il est donc seulement possible de réaliser approximativement les opérateurs unitaires. Le théorème de Solovay-Kitaev $[1,26]$ montre toutefois qu'il est possible de simuler, avec une précision $\varepsilon$, un circuit arbitraire composé de $m$ opérations $C_{\text {NOT }}$ et d'opérations (continues) à un bit à l'aide de $O\left(m \log ^{c}(m / \varepsilon)\right)$ opérations appartenant à l'ensemble discret. Le nombre de portes nécessaires à cette simulation croît de façon polylogarithmique avec $1 / \varepsilon$ et est donc efficace, d'un point de vue algorithmique. Notons que cet ensemble est utile tout particulièrement parce qu'il est compatible avec les techniques du calcul quantique tolérant aux imperfections (fault-tolerant quantum computation) [27]. De plus, d'un point de vue pratique, ce résultat nous apprend qu'un design d'ordinateur quantique n'a pas à appliquer des rotations infinitésimales afin d'être universel. Un ensemble de rotations discrètes suffira.

\subsection{Hamiltoniens et opérations logiques}

Au cours de la discussion précédente, l'attention a été portée sur les opérateurs d'évolution. Si l'on veut réaliser ces opérations logiques sur un système physique, il est alors plus naturel de penser aux hamiltoniens générant ces opérations. En effet, c'est l'hamiltonien du système que l'on peut manipuler au laboratoire. Une question naturelle est donc de déterminer quels hamiltoniens seront suffisamment puissants pour générer un ensemble universel de portes logiques quantiques.

Dans le cas des opérations à un qubit, l'hamiltonien le plus général est

$$
H_{1 q}=B_{x} \sigma_{x}+B_{y} \sigma_{y}+B_{z} \sigma_{z}
$$


Avec un contrôle sur les $B_{i}$, l'Hamiltonien (1.15) peut évidemment générer $S U(2)$. Les coefficients $B_{i}$ dépendent du système physique réalisant le qubit. Pour un spin $1 / 2$, ce sont des champs magnétiques orientés selon différentes directions. Pour d'autres réalisations, ils seront traités comme des champs effectifs en utilisant le langage des pseudo-spins de Feynman.

Pour réaliser une opération à deux qubits, le non-contrôlé par exemple, une interaction entre qubits est nécessaire. La forme du terme d'interaction dépend du système physique réalisant l'ordinateur quantique. Une forme commune à plusieurs architectures est

$$
H_{2 q}=J \sigma_{z} \otimes \sigma_{z}
$$

Un tel terme d'interaction intervient en résonance magnétique nucléaire (RMN) de l'état liquide [28] et pour certaines architectures de qubits supraconducteurs [5]. Cet hamiltonien génère l'opération de changement de phase contrôlé (controlled phase shift)

$$
C_{P}(\gamma)=e^{-i \gamma \sigma_{z} \otimes \sigma_{z} / 2}
$$

Puisque

$$
\begin{aligned}
C_{\text {NOT }}= & e^{-i 3 \pi / 4} R_{x 2}(3 \pi / 2) C_{\mathrm{P}}(3 \pi / 2) R_{\mathrm{z} 2}(\pi / 2) \mathrm{R}_{\mathrm{x} 2}(\pi / 2) \\
& \mathrm{R}_{\mathrm{z} 2}(\pi / 2) \mathrm{R}_{\mathbf{z} 1}(\pi / 2) \mathrm{C}_{\mathrm{P}}(3 \pi / 2),
\end{aligned}
$$

cette opération est équivalente au non-contrôlé. La porte $\mathrm{R}_{\alpha \mathrm{k}}(\theta)$ est une rotation du qubit $k$ d'un angle $\theta$ autour de l'axe $\alpha$ sur la sphère de Bloch.

Avec ce terme d'interaction, l'hamiltonien de l'ordinateur quantique est donc de la forme

$$
H_{S}=\sum_{i=1}^{n}\left[B_{x i} \sigma_{x i}+B_{y i} \sigma_{y i}+B_{z i} \sigma_{z i}\right]+\sum_{\langle i, j\rangle}^{n} J_{i j} \sigma_{z i} \otimes \sigma_{z j} .
$$

De façon plus générale, on écrira

$$
H_{S}=\sum_{i=1}^{n} H_{1 q i}+\sum_{\langle i, j\rangle}^{n} H_{2 q i j} .
$$

Dans ces expressions, le terme d'interaction a été limité aux qubits plus proches voisins puisqu'il est en pratique souvent difficile d'avoir des interactions qubit-qubit à plus longues portées. 


\subsection{QUBITS ENCODÉS}

Puisque les qubits seront couplés à leur(s) environnement(s) ceci ne peut pas être l'hamiltonien total du système. Un hamiltonien plus réaliste s'écrit donc

$$
H=H_{S}+H_{B}+H_{S B}
$$

où $H_{B}$ est l'hamiltonien du bain (environnement) et $H_{S B}$ représente l'interaction entre le système et le bain. Ce dernier terme peut s'écrire comme une somme d'opérateurs du système $\left(\sigma_{i, \alpha}\right)$ et du bain $\left(B_{\alpha}\right)$

$$
H_{S B}=\sum_{i=1}^{N} \sum_{k} \sum_{\alpha} g_{i k \alpha} \sigma_{i \alpha} \otimes B_{k \alpha}
$$

L'indice $k$ distingue les différents modes du bain, $\alpha=x, y, z$ caractérise la forme de l'interaction système-bain et $g_{i k \alpha}$ l'amplitude de ce couplage. Du point de vue des qubits seulement, cette interaction conduit à une évolution non-unitaire. Telle que mentionné précédemment, cette évolution non-unitaire conduit à la décohérence du registre et, par conséquent, à des erreurs dans le calcul.

Une interaction entre le système et le bain apparait inévitable. Heureusement, des techniques capables de protéger l'information quantique des griffes de la décohérence ont été développées.

\subsection{Qubits encodés}

\subsubsection{Corrections quantiques d'erreurs}

Jusqu'à présent, nous avons uniquement porté notre attention sur les qubits physiques. Il est toutefois avantageux, dans plusieurs situations, de regrouper $m$ qubits pour en faire un qubit logique encodé. La première situation où les encodages ont été utilisés en calcul quantique est en relation avec la correction d'erreurs quantiques (quantum error correction, QEC) [29,30].

Afin d'illustrer les techniques de QEC par un exemple simple, considérons la situation où il y a une probabilité finie pour qu'un qubit physique renverse spontanément sa valeur ("bit flip") au cours du calcul. Il est possible de protéger l'information quantique contre ce type d'erreur en encodant chaque qubit logique à l'aide de trois qubits 


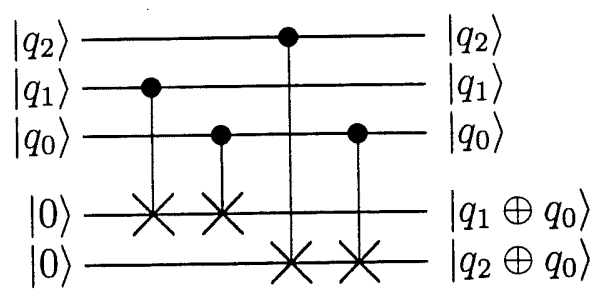

FIG. 1.3: Circuit quantique détectant le renversement d'un bit. Les trois premières lignes sont les qubits du code tandis que les deux dernière sont des qubit auxiliaires. Le 'syndrome' est $\left(q_{1} \oplus q_{0}, q_{2} \oplus q_{0}\right)$, où $\oplus$ est l'addition $\bmod 2$.

physiques [29] :

$$
|0\rangle \mapsto\left|0_{L}\right\rangle=|000\rangle \quad|1\rangle \mapsto\left|1_{L}\right\rangle=|111\rangle .
$$

Les états $\left|0_{L}\right\rangle$ et $\left|1_{L}\right\rangle$ forment la nouvelle base de calcul. Une superposition arbitraire d'états logiques devient dans cette base

$$
a|0\rangle+b|1\rangle \mapsto a|000\rangle+b|111\rangle=a\left|0_{L}\right\rangle+b\left|1_{L}\right\rangle
$$

Une erreur de 'bit flip' sur le premier qubit physique de cette superposition donne

$$
a|000\rangle+b|111\rangle \rightarrow a|100\rangle+b|011\rangle
$$

Sans l'utilisation du code, une telle erreur ruine complètement l'information. Ici, pour corriger l'erreur, il n'est évidemment pas une bonne idée de mesurer directement la superposition d'états, ce que l'on aurait fait avec le code correcteur d'erreurs classique analogue [31]. En effet, (1.25) est un état enchevêtré et la mesure 'effondrerait' complètement la fonction d'onde, ruinant ainsi l'information. Afin d'effectuer la détection d'erreurs, on ajoute, aux trois qubits du code, deux qubits auxiliaires préalablement initialisés dans l'état $|00\rangle$. On applique ensuite le circuit de la figure 1.3 sur ces cinq qubits, puis on mesure les qubits auxiliaires. Le résultat de cette mesure est le syndrome. Il est facile de montrer que celui-ci nous renseigne sur quel qubit le 'bit flip' a eu lieu. Lorsque cette information est connue, il ne reste plus qu'à appliquer l'opération $\mathrm{R}_{\mathbf{x}}(\pi)=\sigma_{x}$ sur le qubit erroné pour le corriger. 


\subsection{QUBITS ENCODÉS}

Pour corriger une erreur arbitraire, il est nécessaire de savoir corriger les erreurs de phase ('phase flip')

$$
a|0\rangle+b|1\rangle \rightarrow a|0\rangle-b|1\rangle .
$$

Ceci peut être fait de façon semblable au cas du bit flip en utilisant à nouveaux trois qubits physiques et un code différent [29]. Avec cette approche, un qubit logique est encodé par neuf qubits physiques ayant pour résultat de le protéger d'une erreur arbitraire sur un de ces qubits physiques. Il existe toutefois des codes plus efficaces. Par exemple, il existe un code à cinq qubits protégeant un qubit encodé d'une erreur arbitraire sur un qubit physique $[14,32]$. Il s'agit de la taille minimale pour un code capable de détecter et corriger une erreur arbitraire sur un qubit $[14,33]$.

Une idée clé concernant les techniques de correction quantique d'erreurs est que les états encodés sont enchevêtrés. Comme en (1.6), aucun qubit physique ne contient alors d'information concernant l'état original. L'information se trouve plutôt dans les corrélations non-locales entre qubits physiques. Ainsi, une erreur sur un qubit physique du code ne produit pas de perte d'information puisque ceux-ci n'ont, individuellement, aucune information. Un second aspect important est que la mesure du syndrome ne nous informe pas sur l'état du qubit encodé; il ne nous informe que sur l'erreur survenue. La mesure des qubits auxiliaires ne perturbe donc pas l'information.

Dans le contexte de ce travail, le message à retenir concernant la correction d'erreurs quantiques est que cette technique nécessite l'habileté de réaliser efficacement des mesures. Puisqu'une erreur devrait être corrigée avant qu'une seconde surgisse, la lecturè des syndromes doit être effectuée très rapidement. Ceci impose des contraintes très sévères sur l'appareil de mesure. En particulier, ceci signifie que ce dernier doit réaliser des mesures 'en un coup' (one-shot measurement) et non pas simplement des mesures faibles (weak measurement). Plus de détails seront donnés sur le processus de la mesure au chapitre 5 .

Remarquons qu'il est connu que toute mesure dans un calcul quantique peut être repoussée à la fin du calcul quantique. Ceci nécessite toutefois de remplacer les opérations contrôlées classiquement par des opérations contrôlées quantiquement (i.e. remplacer des portes à un bit par des portes à deux bits); voir la section $\S 4.4$ de la Réf. [1] pour plus de détails. Cette idée peut être appliquée au QEC pour replacer la mesure des syndromes et les rotations subséquentes par des portes quantiques à deux 


\subsection{QUBITS ENCODÉS}

qubits; voir l'encadré 10.1 de la Réf. [1]. On peut donc assouplir partiellement les contraintes sur l'appareil de mesure. En contrepartie, puisque la correction de chaque erreur demande deux qubits auxiliaires initialisés à $|0\rangle$, il est alors nécessaire d'avoir une réserve de qubits frais à notre disposition. Ceci pose un problème puisqu'une bonne façon d'initialiser un qubit est de le mesurer. On retrouve alors les mêmes contraintes pour l'appareil de mesure. Une autre approche à ce problème de qubits frais est d'initialiser au début du calcul un grand nombre de qubits auxiliaires et de les distribuer au moment et au lieu où ils sont nécessaires au cours du calcul. Des idées similaires ont aussi été abordées dans la référence Réf. [19]. Notons qu'il serait intéressant d'appliquer à ce problème de la distribution de qubits frais les techniques d'optimisation d'algorithmes quantiques décrites à la section $§ 1.7$ de ce document.

On mentionne finalement que la procédure de correction décrite ici suppose qu'il n'y a pas d'erreurs sur les qubits auxiliaires. Une telle situation serait désastreuse puisqu'une erreur sur un qubit auxiliaire en entraîne deux sur les qubits du code. Heureusement, les techniques plus sophistiquées du calcul tolérant aux imperfections peuvent corriger cette lacune importante [27].

\subsubsection{Sous-espaces sans décohérence}

Puisque des actions doivent être effectuées à chaque étape pour détecter et corriger les erreurs, la correction d'erreurs quantiques est une approche active à la réduction d'erreurs. De plus, elle repose sur l'hypothèse que les erreurs affectent les qubits de façon indépendante (certains codes peuvent toutefois protéger un qubit encodé de plus d'une erreur $[34,35])$. Une stratégie complémentaire qui évite passivement les erreurs a aussi été développée $[36,37]$. Cette stratégie est basée sur l'encodage de l'information dans un sous-espace sans décohérence (decoherence free-subspaces, DFS).

Les conditions pour qu'un sous-espace ne subisse pas les effets de la décohérence sont simples à énoncer. On tire celles-ci presque textuellement de la référence [38]. D'abord, supposons que les constantes de couplage dans l'hamiltonien système-bain (1.22) sont indépendantes de l'index du qubit : $g_{i k \alpha} \equiv g_{k \alpha}$. Ceci correspond à la situation où les qubits interagissent tous avec le même environnement. L'hamiltonien 
système-bain peut alors se réécrire sous la forme

$$
H_{S B}=\sum_{\alpha} S_{\alpha} \otimes B_{\alpha}
$$

où $S_{\alpha} \equiv \sum_{i} \sigma_{i \alpha}$ et $B_{\alpha} \equiv \sum_{k} g_{k \alpha} B_{k \alpha}$.

Maintenant, soit $\tilde{\mathcal{H}}_{S}$ un sous-espace de l'espace d'Hilbert du système $\mathcal{H}_{S}$ avec comme états de base $\{|\tilde{\phi}\rangle\}$. Ces états font intervenir plusieurs qubits. L'évolution dans ce sous-espace sera unitaire, et donc $\{|\tilde{\phi}\rangle\}$ un DFS, si et seulement si (i)

$$
S_{\alpha}|\tilde{\phi}\rangle=c_{\alpha}|\tilde{\phi}\rangle \quad \forall \quad|\tilde{\phi}\rangle \text { et } \alpha,
$$

où $c_{\alpha}$ est un nombre complexe. (ii) La partie 'système' de l'hamiltonien (celle qui génère le calcul) ne mélange pas les états de ce sous-espace aux états à l'extérieur de ce sous-espace. (iii) Le système et le bain sont initialement découplés : $\rho(0)=\rho_{S}(0) \otimes \rho_{B}$ (il s'agit d'une supposition standard). Un exemple illustrant simplement ces idées est présenté ultérieurement.

Si ces conditions sont satisfaites, alors encoder les qubits physiques dans la base $\{|\tilde{\phi}\rangle\}$ protégera l'information de l'environnement. Les états $|\tilde{\phi}\rangle$ jouent donc le rôle d'états logiques pour les qubits encodés. Cette approche est passive puisque, une fois l'information encodée dans le DFS, elle ne peut être perturbée par l'environnement. Aucune étape de détection ou de correction n'est donc nécessaire ici.

Il est important d'insister sur le fait que cette approche ne fonctionnera que dans les cas particuliers où $g_{i k \alpha} \equiv g_{k \alpha}$. Il s'agit du modèle de 'décohérence collective' [39]. Cette situation est en opposition au cas de la QEC où les erreurs sont supposées être complètement indépendantes. Ces deux techniques peuvent être utilisées ensemble afin de tirer profit de leurs avantages respectifs [40]. Notons que les techniques d'encodage dans un DFS ont été testées expérimentalement avec succès dans différents systèmes physiques [41-43].

\subsubsection{Universalité encodée}

Le codage peut être encore utile dans un autre contexte. Imaginons que l'hamiltonien d'un registre de $n$ qubits est

$$
H_{S}=\frac{1}{2} \sum_{i} B_{z i} \sigma_{z i}+\sum_{i} J_{z i j} \sigma_{z i} \sigma_{z j}+\sum_{i, j} J_{x y i j}\left(\sigma_{x i} \sigma_{x j}+\sigma_{y i} \sigma_{y j}\right) .
$$




\subsection{QUBITS ENCODÉS}

où nous supposons avoir un contrôle sur $B_{z i}, J_{z i j}$ et $J_{x y i j}$. Ce type d'hamiltonien décrit plusieurs architectures possibles d'ordinateurs quantiques : système basé sur l'effet Hall quantique [44], électrons sur hélium liquide [45], certaines architectures utilisant les points quantiques [46] et les architectures basées sur le spin nucléaire d'impuretés dans le silicium [47]. Dans ces systèmes, il est aussi possible, en principe, de contrôler un terme de la forme $B_{x} \sigma_{x}$ sur chacun des qubits. Toutefois, ceci est en pratique difficile à réaliser et l'opération résultante sera lente dans la plupart des cas. L'hamiltonien (1.29) semble donc plus réaliste pour ces architectures. Malheureusement, (1.29) n'est pas universel puisqu'il ne permet pas de générer $S U(2)$ sur les qubits physiques. C'est ici que les codes peuvent à nouveau être utiles.

Exprimons tout d'abord le terme $\sigma_{x i} \sigma_{x j}+\sigma_{y i} \sigma_{y j}$ dans la base de calcul $\{|00\rangle,|01\rangle$, $|10\rangle,|11\rangle\}$ des qubits $i$ et $j$

$$
\frac{1}{2}\left(\sigma_{x i} \sigma_{x j}+\sigma_{y i} \sigma_{y j}\right)=\left(\begin{array}{cccc}
0 & & & \\
& 0 & 1 & \\
& 1 & 0 & \\
& & & 0
\end{array}\right)=\left(\begin{array}{lll}
0 & & \\
& \bar{\sigma}_{x i j} & \\
& & 0
\end{array}\right)
$$

Ce terme agit comme $\sigma_{x}$ dans le sous-espace $\{|01\rangle,|10\rangle\}$ et laisse le sous-espace $\{|00\rangle,|11\rangle\}$ invariant. De plus, cette opération ne mélange pas ces deux sous-espaces. De ce fait, en encodant les qubits logiques comme

$$
\begin{aligned}
& \left|0_{L}\right\rangle=|01\rangle \\
& \left|1_{L}\right\rangle=|10\rangle,
\end{aligned}
$$

nous savons comment réaliser un $\bar{\sigma}_{x}$ encodé avec l'hamiltonien (1.29), ce que l'on ne pouvait faire avec les qubits physiques. Le " - " au-dessus de $\bar{\sigma}_{x}$ est utilisé pour insister sur le fait qu'il s'agit d'une opération encodée agissant sur un qubit encodé. Pour que cette construction soit utile, il doit être possible d'appliquer toutes les opérations logiques sur ce sous-espace. Il est facile de montrer comment cela est possible. Premièrement, considérons

$$
B_{z i} \sigma_{z i}+B_{z j} \sigma_{z j}=\left(\begin{array}{llll}
B_{z i}+B_{z j} & & & \\
& B_{z i}-B_{z j} & & \\
& & -\left(B_{z i}-B_{z j}\right) & \\
& & & -\left(B_{z i}+B_{z j}\right)
\end{array}\right) \text {. }
$$




\subsection{QUBITS ENCODÉS}

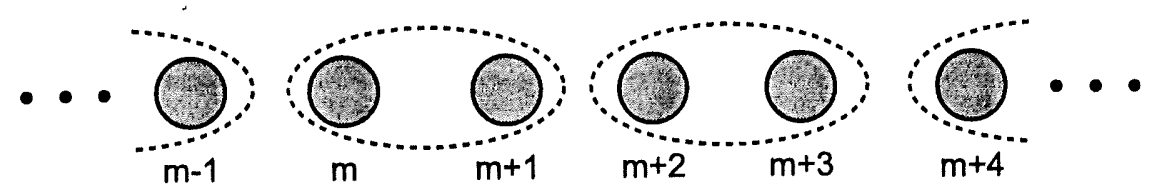

FIG. 1.4: Qubits physiques alignés sur un même axe. Les cercles représentent les qubits physiques et un ovale indique une paire de qubits physiques formant un qubit logique.

Avec $B_{z i}-B_{z j} \neq 0$, ceci agit comme $\bar{\sigma}_{z}$ sur $\left\{\left|0_{L}\right\rangle,\left|1_{L}\right\rangle\right\}$ et de surcroît, ne mélange pas l'espace de calcul avec le reste de l'espace d'Hilbert. Les opérations encodées (1.30) et (1.32) générent $\overline{S U(2)}$ sur le sous-espace encodé.

Le dernier ingrédient manquant est une opération non-triviale sur deux qubits encodés et donc agissant sur quatre qubits physiques. Afin de montrer comment une telle opération est possible, supposons d'abord que les qubits sont fabriqués de façon à être alignés sur un même axe. Tel qu'illustré à la figure 1.4, Les qubits $m$ et $m+1$ sont donc plus proches voisins tandis que $m$ et $m+2$ sont seconds plus proches voisins. Le code est construit de telle sorte que les paires $(m, m+1)$ et $(m+2, m+3)$ forment chacune un qubit encodé. Puisque $\sigma_{z m+1} \sigma_{z m+2}$ agit comme $-\overline{\sigma_{z} \sigma_{z}}$ sur $\left\{\left|0_{L}\right\rangle,\left|1_{L}\right\rangle\right\} \otimes$ $\left\{\left|0_{L}\right\rangle,\left|1_{L}\right\rangle\right\}$, afin de réaliser une opération encodée à deux qubits il est donc suffisant de fixer tout les coefficients sauf $J_{z, m+1, m+2}$ à zéro. Cette interaction nous permet de réaliser une opération de phase contrôlée encodée $C_{P}(1.17)$, ce qui complète la preuve d'universalité. Cette construction a été décrite dans la référence [48] en utilisant un 'mapping' intéressant, mais plus complexe, des qubits vers les 'parafermions'. Il est intéressant de préciser que pour mettre en application toutes les idées présentées cidessus, seul le couplage au plus proche voisin entre qubits physiques est nécessaire. Ceci réduit les exigences techniques reliées à la réalisation d'un ordinateur quantique. Cette simplification est due à l'auteur de ce document.

Afin de pouvoir utiliser ces idées, il est nécessaire de donner une prescription pour l'initialisation d'un registre quantique dans un état encodé standard (par exemple, $\left.\left|0_{L} 0_{L} \cdots 0_{L}\right\rangle\right)$. Ceci est plus compliqué que dans le cas non encodé puisque les états logiques (1.31) sont justement ceux que l'hamiltonien (1.29) ne peut atteindre par des manipulations unitaires. Toutefois, une observation utile est que le fondamental de $\sigma_{x} \sigma_{x}+\sigma_{y} \sigma_{y}$ est $\left(\left|0_{L}\right\rangle-\left|1_{L}\right\rangle\right) / \sqrt{2}$. Ainsi, ajustant tous les coefficients $B_{z}$ et $J_{z}$ à zéro et les $J_{x y}$ non-nuls, le système devrait éventuellement relaxer vers son état fondamental 


\subsection{QUBITS ENCODÉS}

qui est dans le sous-espace encodé [48]. La lecture peut être réalisée en mesurant un des qubits physiques du code dans la base $\{|0\rangle,|1\rangle\}$ usuelle. Une approche alternative mais plus complexe est décrite dans la Réf. [48].

En plus de rendre universel l'hamiltonien (1.29), le code (1.31) a une propriété additionnelle très intéressante : il s'agit d'un DFS pour les erreurs de phase. En effet, supposons que la seule action de l'environnement soit de déphaser les qubits physiques de la façon suivante :

$$
\begin{aligned}
& \left|0_{i}\right\rangle \mapsto\left|0_{i}\right\rangle \\
& \left|1_{i}\right\rangle \mapsto e^{i \phi}\left|1_{i}\right\rangle,
\end{aligned}
$$

où la phase $\phi$ est indépendante de l'index $i$ du qubit. Tandis qu'une superposition arbitraire d'un qubit physique va perdre sa cohérence sous l'action de cet environnent, une superposition de qubits logiques encodés dans le sous espace (1.31) ne sera pas affectée puisque seule une phase globale interviendra

$$
a\left|0_{L}\right\rangle+b\left|1_{L}\right\rangle=a|01\rangle+b|10\rangle \mapsto a e^{i \phi}|01\rangle+b e^{i \phi}|10\rangle=e^{i \phi}\left(a\left|0_{L}\right\rangle+b\left|1_{L}\right\rangle\right) .
$$

Ce code a été utilisé dans l'expérience décrite à la référence [42] pour protéger l'information encodée dans une paire d'ions contre les fluctuation d'un champ magnétique parasite.

Cette idée de prendre un hamiltonien non universel dans l'espace d'Hilbert total et de trouver un sous-espace plus petit dans lequel il est universel est appelée universalité encodée. Un aspect intéressant de cette approche est qu'il est possible de ne considérer que les 'talents naturels' d'un système physique plutôt que d'imposer des termes de contrôle supplémentaires impliquant des difficultés technologiques. L'universalité encodée a été étudiée en relation avec différents hamiltoniens et systèmes physiques [38,49-51]. En particulier, elle a été étudiée en relation avec les qubits supraconducteurs de phase où il a été montré comment certaines opérations difficiles à réaliser ne sont pas nécessaires pour atteindre l'universalité [52]. Dans ce cas, le code utilise le concept de 'bus qubit' fixé dans l'état $|1\rangle$. Cet article est reproduit à l'annexe $\mathrm{C}$ de ce document.

Une idée clé à retenir de cette discussion est qu'un qubit logique n'a pas à être un système physique à deux niveaux. Puisque l'information (quantique) est une ressource fongible, il est possible d'utiliser d'autres systèmes physiques pour réaliser un 
qubit logique. Ce concept est un des développements plus récents allant au-delà du paradigme standard. Pour un survol de certaines autres façons d'aller au-delà de ce paradigme, consulter la référence [20].

\subsection{Algorithmes quantiques}

Maintenant que l'on sait, en principe, stabiliser l'information quantique et faire du calcul quantique universel, comment peut-on tirer profit des superpositions d'états et de l'enchevêtrement afin de réaliser des calculs utiles plus rapidement qu'avec un ordinateur classique? Quels sont les calculs pouvant êtres accélérés par ces effets quantiques?

Il peut être surprenant de savoir que ces questions sont encore ouvertes. Premièrement, ce qui donne à un ordinateur sa puissance de calcul n'est pas encore bien compris [18]. On sait par exemple que certains calculs ne peuvent pas être accélérés quantiquement [53]. De plus, il n'est même pas encore connu si les ordinateurs quantiques sont vraiment plus puissants que leur contrepartie classique, voir $\S 1.4 .5$ de la Réf. [1] pour une discussion d'introduction aux classes de complexité impliquées. Il y a. toutefois certaines évidences en cette faveur. Par exemple, on sait que les ordinateurs quantiques sont bons dans le calcul des transformées de Fourier (quantique) [54], pour chercher dans des bases de données désordonnées [55] et pour simuler des systèmes physiques $[56,57]$.

Quoique ces trois algorithmes puissent être appliqués à une grande variété de problèmes, il est un peu troublant de constater que ce sont les seuls algorithmes quantiques connus. Ce qui est encore plus surprenant est que de cette (courte) liste, l'algorithme le plus récent (la recherche quantique) a été découvert en 1996. La dernière découverte majeure du côté des algorithmes quantiques date donc d'il y a 8 ans (au moment d'écrire ce document). Ceci montre clairement que "d'écrire" un algorithme quantique n'est pas une tâche facile. Cette difficulté est due en partie à notre manque d'intuition pour des choses aussi étranges que superpositions d'états et enchevêtrement. Une autre difficulté avec les algorithmes quantiques est l'extraction de l'information. Un ordinateur quantique peut calculer simultanément un nombre exponentiel de résultats (il s'agit du parallélisme quantique) mais, à la fin, on ne peut 
en mesurer qu'un seul.

Comme exemple spécifique de cette difficulté à extraire l'information, considérons l'algorithme d'estimation de phase. Comme la plupart des algorithmes quantiques, celui-ci utilise la transformée de Fourier quantique (quantum Fourier transform, QFT) et cet exemple servira donc d'introduction à cette transformation quantique importante.

\subsubsection{Transformée de Fourier quantique}

L'algorithme d'estimation de phase a d'abord été introduit par Kitaev [58] et ensuite reformulé par Cleve et al. [59]. Le but de cet algorithme est d'estimer les valeurs propres d'un opérateur unitaire. Puisqu'il utilise la QFT, revoyons d'abord cette transformation. La QFT agit sur un registre de $n$ qubits indexé $\mid n-1, n-$ $2, \cdots, 1,0\rangle$ de la façon suivante

$$
\mathrm{F}_{\mathrm{n}}:|x\rangle \mapsto \frac{1}{2^{n / 2}} \sum_{y=0}^{2^{n}-1} e^{2 \pi i \frac{x y}{2^{n}}}|y\rangle .
$$

Cette transformation peut être réalisée à l'aide de deux opérations logiques de base [60] : la transformation de Hadamard $H$ et l'opération de phase conditionnelle. Suivant la notation de Shor [61], la transformation de Hadamard sera ici notée $A_{j}$, où l'indice $j$ indique que l'opération agit sur le qubit $j$. L'opération de phase conditionnelle $\mathrm{B}_{\mathrm{jk}}$, agissant sur les qubits $j$ et $k$ est

$$
\mathrm{B}_{\mathrm{jk}}=\left(\begin{array}{cccc}
1 & & & \\
& 1 & & \\
& & 1 & \\
& & & e^{i \theta_{j k}}
\end{array}\right),
$$

avec $\theta_{j k}=\pi / 2^{k-j}$. Dans le cas d'un registre de cinq qubits, la séquence suivante réalise la $\mathrm{QFT}$

$$
A_{0} B_{01} A_{1} B_{02} B_{12} A_{2} B_{03} B_{13} B_{23} A_{3} B_{04} B_{14} B_{24} B_{34} A_{4}
$$

Comme toujours, les opérations sont appliquées en commençant par la droite. Le circut quantique correspondant est présenté à la figure 1.5. La transformation $F_{5}$ est 


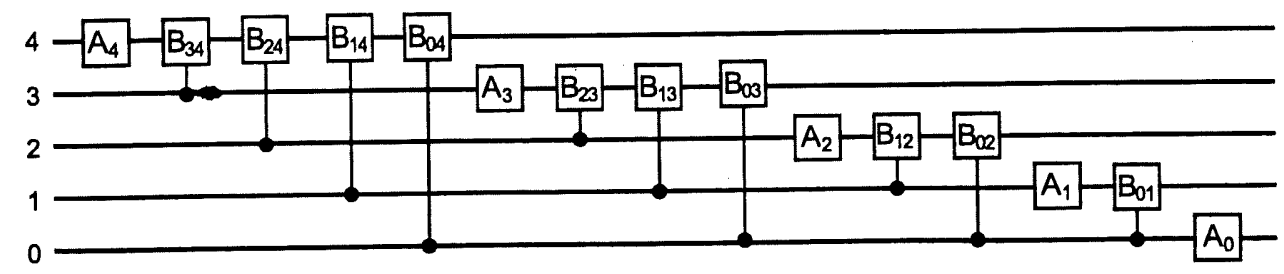

FIG. 1.5: Circuit quantique réalisant $F_{5}$, la transformée de Fourier quantique sur un registre de 5 qubits. Le résultat de ce circuit est l'inverse (bit-reversal) de la transformée de Fourier (1.37).

donc réalisée avec 15 opérations logiques. De façon plus générale, $n$ opérations à un qubit et $n(n-1) / 2$ opérations à deux qubits sont requises pour réaliser $F_{n}$.

\subsubsection{Algorithme d'estimation de phase}

Intéressons-nous maintenant à l'algorithme d'estimation de.phase. Le but est d'estimer les valeurs propres $\left\{\mu_{p}\right\}$ d'un opérateur unitaire $U$. À ces valeurs propres sont associés les vecteurs propres $\left\{\left|\psi_{p}\right\rangle\right\}$. Physiquement, il est souvent plus intéressant de trouver les valeurs propres $\left\{\lambda_{p}\right\}$ d'un hamiltonien $H$. L'algorithme d'estimation de phase nous permet aussi de le faire puisque qu'il est possible de travailler avec $\mathrm{U}=e^{i H}$ qui a les mêmes vecteurs propres que $H$ et les valeurs propres $\left\{e^{2 \pi i \mu_{p}}\right\}$, où $\lambda_{p}=2 \pi \mu_{p}$. Au meilleur de ma connaissance, l'application de l'algorithme d'estimation de phase à des problèmes d'intérêts physiques a été suggérée pour la première fois par Abrams et Lloyd [62] et, indépendamment, par le présent auteur dans un rapport non-publié [63]. Depuis, des idées similaires ont été publiées par différents auteurs et avec plus ou moins de détails supplémentaires $[64,65]$.

L'algorithme utilise un ordinateur quantique à deux registres. Le premier registre est formé de $m$ qubits et le second de $n=\log _{2} N$ qubits. Ici, $m$ est le nombre de bits de précision avec laquelle les valeurs propres seront estimées (et peut donc être ajusté selon les besoins) tandis que $N=2^{n}$ est la taille de la matrice hermitique $H$.

La transformation contrôlée suivante sera nécessaire

$$
\mathrm{C}_{U}^{\mathrm{k}}\left|x_{k}\right\rangle\left|\psi_{p}\right\rangle=e^{2 \pi i x_{k} \mu_{p}}\left|x_{k}\right\rangle\left|\psi_{p}\right\rangle
$$

Dans cette expression, $\left|x_{k}\right\rangle$ est un registre de un qubit de sorte que $x_{k} \in\{0,1\}$. 


\subsection{ALGORITHMES QUANTIQUES}

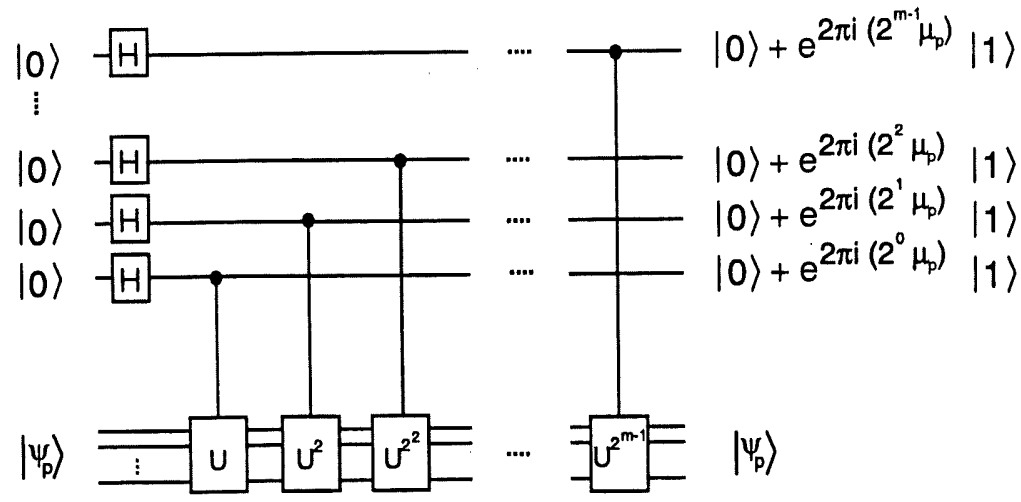

FIG. 1.6: Circuit quantique pour l'estimation de phase (Adaptée de la Réf. [59].)

L'opération $C_{U}^{k}$ applique donc une phase $2 \pi i \mu_{p}$ seulement si le bit de contrôle $k$ est dans l'état $|1\rangle$.

Les étapes de l'algorithme d'estimation de phase sont les suivantes. Premièrement, les $m$ qubits du premier registre sont initialisés dans l'état $|0\rangle$ et le second registre est préparé dans un état propre $\left|\psi_{p}\right\rangle$ de U. Ensuite, la transformée de Fourier du premier registre est effectuée. Puisque tous les qubits de ce registre sont dans l'état $|0\rangle$, ceci se réduit à appliquer une transformation de Hadamard indépendamment sur chaque qubit, comme à la figure 1.6. On applique maintenant $\mathrm{C}_{\mathrm{U}^{\mathrm{2}} \mathrm{r}-\mathrm{r}}^{\mathrm{avec}}$ le $r^{\mathrm{e}}$ qubit du premier registre comme bit de contrôle et on répète cette opération pour chacun des $m$ qubits de ce registre. Le résultat est alors

$$
\begin{aligned}
|0\rangle|0\rangle \cdots|0\rangle\left|\psi_{p}\right\rangle \rightarrow & \frac{1}{\sqrt{2}}\left(|0\rangle+e^{2 \pi i 2^{m-1} \mu_{p}}|1\rangle\right) \frac{1}{\sqrt{2}}\left(|0\rangle+e^{2 \pi i 2^{m-2} \mu_{p}}|1\rangle\right) \\
& \cdots \frac{1}{\sqrt{2}}\left(|0\rangle+e^{2 \pi i \mu_{p}}|1\rangle\right)\left|\psi_{p}\right\rangle \\
= & \frac{1}{2^{m / 2}} \sum_{y=0}^{2^{m}-1} e^{2 \pi i \mu_{p} y}|y\rangle\left|\psi_{p}\right\rangle .
\end{aligned}
$$

Pour arriver à ce résultat, un état propre $\left|\psi_{p}\right\rangle$ de $U$ a été utilisé. Ceci suppose que l'on connaît cet état et que l'on sait comment le préparer. Cela ne sera généralement pas le cas. Heureusement, il est suffisant de préparer le second registre dans un état arbitraire $|a\rangle$ de la base de calcul, $a \in\{0,1\}^{\otimes n}$. Cet état peut évidemment être 
développé dans la base $\left\{\left|\psi_{p}\right\rangle\right\}$

$$
|a\rangle=\sum_{p}\left|\psi_{p}\right\rangle\left\langle\psi_{p} \mid a\right\rangle \equiv \sum_{p} \alpha_{p}\left|\psi_{p}\right\rangle
$$

Utilisant l'état (1.40) comme état initial pour le second registre, on obtient alors

$$
|0\rangle|0\rangle \cdots|0\rangle|a\rangle \rightarrow \frac{1}{2^{m / 2}} \sum_{p} \alpha_{p} \sum_{y=0}^{2^{m}-1} e^{2 \pi i \mu_{p} y}|y\rangle\left|\psi_{p}\right\rangle .
$$

Afin d'extraire une valeur propre, on applique ensuite une QFT inverse au premier registre (cette étape n'est pas présentée à la figure 1.6). Cette opération agit comme la QFT (1.35) mais dans la direction opposée. Son action est donc de 'transférer' la phase $x$ de l'exponentiel $e^{2 \pi i \frac{x y}{2^{n}}}$ au vecteur d'état $|x\rangle$, ce qui est exactement ce dont on a besoin ici. En effet, après avoir réalisé cette opération, le résultat est

$$
\frac{1}{2^{m}} \sum_{p} \sum_{x=0}^{2^{m}-1} \sum_{y=0}^{2^{m}-1} \alpha_{p} e^{-2 \pi i \frac{x_{y}}{2^{m}}} e^{2 \pi i \mu_{p} y}|x\rangle\left|\psi_{p}\right\rangle
$$

La mesure du premier registre, $|x\rangle$, donnera alors le meilleur estimé sur $m$ bits de $\mu_{p}$ et ce avec une probabilité d'au moins $\left|\alpha_{p}\right|^{2} \frac{4}{\pi^{2}}$. Afin de vérifier que cela est bien le cas, supposons que la fraction binaire $b_{p} / 2^{m}=b_{1} / 2+b_{2} / 4+\ldots+b_{m} / 2^{m} \equiv 0 . b_{1} b_{2} \ldots b_{m}$ est le meilleur estimé sur $m$ bits de $\mu_{p}$. On a donc que, $\mu_{p}=b_{p} / 2^{m}+\delta$, avec $0<\delta \leq 1 / 2^{m+1}$. Insérant ce résultat dans (1.42), on obtient

$$
\frac{1}{2^{m}} \sum_{p} \sum_{x=0}^{2^{m}-1} \sum_{y=0}^{2^{m}-1} \alpha_{p} e^{2 \pi i\left(b_{p}-x\right) y / 2^{m}} e^{2 \pi i \delta y}|x\rangle\left|\psi_{p}\right\rangle .
$$

Si $x$ est le meilleur estimé de $\mu_{p}$ sur $m$ bits, alors $x=b_{p}$ et (1.43) peut s'écrire

$$
\frac{1}{2^{m}} \sum_{p} \sum_{y=0}^{2^{m}-1} \alpha_{p} e^{2 \pi i \delta y}\left|b_{p}\right\rangle\left|\psi_{p}\right\rangle
$$

En sommant la série géométrique, on obtient finalement

$$
\frac{1}{2^{m}}\left(\frac{1-e^{2 \pi i \delta 2^{m}}}{1-e^{2 \pi i \delta}}\right) \sum_{p} \alpha_{p}\left|b_{p}\right\rangle\left|\psi_{p}\right\rangle
$$


Puisque

$$
\left|\frac{1}{2^{m}}\left(\frac{1-e^{2 \pi i \delta 2^{m}}}{1-e^{2 \pi i \delta}}\right)\right|^{2} \geq \frac{4}{\pi^{2}}
$$

(voir l'équation (5.4) de [59] pour les détails), suite à une mesure du premier registre, la probabilité qu'on obtienne le meilleur estimé de $\mu_{p}$ sur $m$ bits est au moins $4\left|\alpha_{p}\right|^{2} / \pi^{2}$.

La procédure décrite ci-haut peut être utilisée pour obtenir les valeurs propres de matrices unitaires arbitraires. De plus puisque dans (1.45) les registres sont enchevêtrés, si la mesure du premier registre produit le résultat $\left|b_{p^{\prime}}\right\rangle$, alors le second registre sera projeté dans l'état $\left|\psi_{p^{\prime}}\right\rangle$. Ainsi, cet algorithme peut être utilisé pour préparer les vecteurs propres de $H$. Malheureusement, il semble difficile d'extraire ces états. En effet, suite à la mesure du premier registre, la lecture du second registre dans la base de calcul ne donnera qu'un entier $x \in\{0,1\}^{\otimes n}$ avec une probabilité $\left|\left\langle x \mid \psi_{p^{\prime}}\right\rangle\right|^{2}$. L'ordinateur quantique peut être utilisé pour calculer certaines quantités mais les extraire peut être difficile en raison de 'l'effondrement de la fonction d'onde'. Certaines fonctions de corrélation peuvent toutefois être calculées [65].

Quelques commentaires s'imposent. Premièrement, la probabilité d'obtenir le meilleur estimé à $m$ bits peut être amplifiée en utilisant $m^{\prime}>m$ et en ne demandant uniquement que $m$ bits de précision. Voir l'annexe $\mathrm{C}$ de la référence [59] pour plus de détails. Une remarque importante est que $\left|\alpha_{p}\right|^{2}$ sera inversement proportionnel à $N$. Ainsi, l'algorithme devient moins efficace avec une augmentation de la taille de la matrice. Si des estimés des $\left\langle\psi_{p}\right\rangle$ sont connus, il est alors avantageux d'utiliser cette connaissance dans la préparation du second registre de façon à amplifier le recouvrement $\alpha_{p}$.

Considérons maintenant les ressources en temps et en espace (i.e. nombre de bits) requises par cet algorithme. Premièrement, afin d'obtenir toutes les valeurs propres il est nécessaire de répéter l'algorithme au moins $O(N)$ fois. De plus, comme on le voit à la figure 1.6, $m$ applications de la transformation d'Hadamard et de la porte $\mathrm{U}^{2^{k}}$ contrôlée sont nécessaires. Finalement, la transformée de Fourier inverse requiert $O\left(m^{2}\right)$ opérations. Ainsi, obtenir un estimé des valeurs propres d'une matrice de taille $N \times N$ demande $O\left(N m^{2}\right)$ opérations agissant sur $m+\log _{2} N$ qubits.

Cet estimé rudimentaire suppose que les opérations U-contrôlées sont réalisées en un temps constant. Ces opérations sont en réalité construites à partir d'opérations 
élémentaires, c'est-à-dire à partir d'opérations prises dans $\left\{S U(2), C_{\text {NOT }}\right\}$. Le temps requis pour réaliser $\mathrm{C}_{\mathrm{U}^{2 m-r}}^{r}$ dépendra donc du nombre de bits sur lequel l'opération agit. De même, puisqu'il n'existe pas de procédure générale pour décomposer de façon optimale une opération arbitraire à l'aide de portes élémentaires [66], beaucoup de complexité peut être cachée dans l'application de ces portes.

Dans certains cas, il peut donc être plus avantageux d'appliquer $2^{m-r}$ fois la transformation $\mathrm{C}_{U}^{r}$ en utilisant le bit de contrôle $r$ plutôt que d'appliquer une fois la transformation $\mathrm{C}_{\mathrm{U}^{2 m-r}}^{r}$. Ceci change l'estimation des ressources requises puisque $2^{m}-1$ opérations contrôlées doivent maintenant être appliquées. En utilisant cette stratégie, le coût total en temps est donc $O\left(2^{m} \times N\right)$ mais une seule décomposition de $C_{U}$ à l'aide de portes élémentaires doit être effectuée par matrice $H$ ou $U$. L'algorithme est donc exponentiel dans le nombre $m$ de bits de précision demandée. Puisque $m$ sera généralement plus petit que $N$, ceci ne devrait pas être dramatique.

\subsection{Optimisation d'algorithmes quantiques}

Il a déjà été mentionné à la section $\$ 1.5$ que le fait de limiter l'interaction entre qubits au premier voisin seulement peut être avantageux du point de vue de la fabrication de l'ordinateur quantique. Toutefois, les circuits utilisés à la section précédente ne tiennent clairement pas compte de telles limitations pratiques. Par exemple, le circuit de QFT présenté à la figure 1.5 fait interagir toutes les paires de qubits.

En pratique ces limitations basées sur des aspects technologiques devront être considérées. Puisque pour un ordinateur quantique le court temps de cohérence sera une contrainte importante, on s'intéresse dans cette section à l'optimisation des algorithmes quantiques en tenant compte de ces restrictions imposées par des aspects pratiques. On considérera le cas le plus contraignant, mais aussi le plus simple à réaliser physiquement : les qubits disposés sur un même axe, comme à la figure 1.4, et ayant des interactions au premier voisin seulement. L'optimisation dans une telle configuration est possible en tirant profit de la possibilité de réaliser les opérations logiques sur différents qubits simultanément. Cette particularité est commune à plusieurs architectures d'ordinateurs quantiques. Afin d'illustrer la méthode, on s'intéressera ici au cas important de la transformée de Fourier quantique. Cette section est basée sur 
des résultats publiés à la référence [67].

Notons que l'optimisation de circuits quantiques par parallélisation a été étudiée aux références [68] et [69] mais sans tenir compte de la portée limitée de l'interaction qubit-qubit. Une étude du 'coût réel en temps' de la QFT incluant une telle contrainte a été présentée à la référence [70] mais sans tirer profit de plusieurs améliorations possibles, incluant le parallélisme.

\subsubsection{Optimisation des permutations}

Afin de procéder à l'optimisation de la QFT, une prescription pour la réalisation de $A_{j}$ et $B_{j k}$ à l'aide d'un ensemble universel d'opérations élémentaires est nécessaire. Une telle description limite la porté de l'optimisation aux architectures ayant cet ensemble particulier de portes dans leur répertoire. L'application de ces idées à d'autres architectures est toutefois simple. On utilisera ici l'ensemble complet

$$
\mathrm{R}_{\mathrm{xj}}(\theta)=e^{-i \sigma_{x j} \theta / 2} ; \quad \mathrm{R}_{\mathrm{zj}}(\phi)=e^{-i \sigma_{z j} \phi / 2} ; \quad \mathrm{C}_{\mathrm{pjk}}(\zeta)=e^{-i \sigma_{z j} \otimes \sigma_{z k} \zeta / 2}
$$

agissant sur les qubits $j$ et $k$. Cet ensemble est intéressant puisqu'il peut être réalisé par plusieurs architectures basées sur les qubits supraconducteurs $[5,71,72]$ ainsi qu'en RMN [73, 74].

Ces opérations élémentaires réalisent les opérations logiques $A_{j}$ et $B_{j k}$ (sur deux qubits adjacents) à l'aide des séquences suivantes :

$$
\begin{aligned}
\mathrm{A}_{\mathrm{j}} & =i \mathrm{R}_{\mathrm{zj}}(\pi / 2) \mathrm{R}_{\mathrm{xj}}(\pi / 2) \mathrm{R}_{\mathrm{zj}}(\pi / 2) ; \\
\mathrm{B}_{\mathrm{jk}} & =e^{i \theta_{k-j+1}} \mathrm{R}_{\mathrm{zj}}\left(\theta_{k-j+1}\right) \mathrm{R}_{\mathrm{zk}}\left(\theta_{k-j+1}\right) \mathrm{C}_{\mathrm{pjk}}\left(\theta_{k-j+1}\right) .
\end{aligned}
$$

La phase $e^{i \theta_{k-j+1}}$ dépend des qubits sur lesquels l'opération $B_{\mathrm{jk}}$ agit mais ne dépend pas de leur état logique. Il s'agit donc d'une phase globale sans importance et peut être ignorée.

Afin de réaliser le circuit de la figure 1.5, il est nécessaire d'appliquer des portes $B_{j k}$ sur des qubits n'étant pas adjacents. Dans une architecture unidimensionnelle, cela implique qu'il faut permuter de façon récursive l'état de qubits adjacents afin de positionner l'état des qubits devant interagir dans des qubits voisins. Pour des qubits initialement aux positions $j$ et $k$ dans le registre, $|j-k|-1$ opérations de permutation 


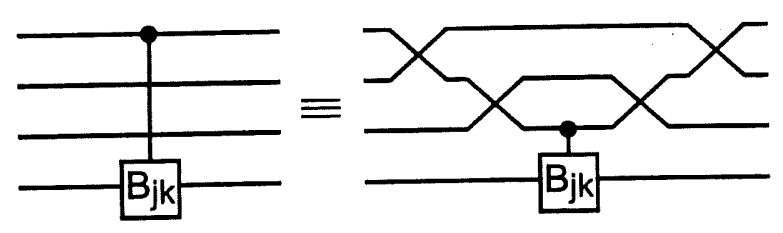

(a)

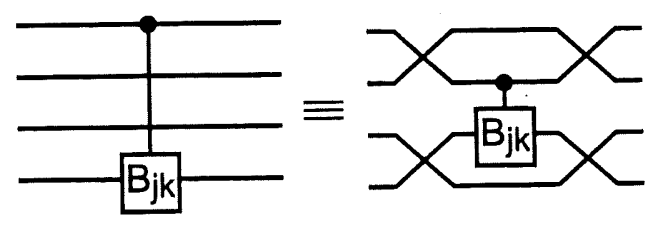

(b)

FIG. 1.7: a) Utilisation répétée de l'opération SWAP afin d'appliquer une opération contrôlée sur des qubits initialement non-adjacents. b) Circuit équivalent utilisant des opérations simultanées. La stratégie a) a été utilisée dans la référence [70] afin d'estimer le coût en temps de la porte $F_{n}$.

sont requises afin de juxtaposer les états et, à nouveau, $|j-k|-1$ afin de ramener les états à leur position d'origine, figure $1.7 \mathrm{a}$ ).

Une permutation entre l'état des qubits aux positions $r$ et $s$ est réalisée par la séquence suivante de portes $C_{\text {NOT }}$

$$
\mathrm{S}_{\mathrm{Wrs}}=\mathrm{C}_{\text {NOTrs }} \mathrm{C}_{\text {NOTsr }} \mathrm{C}_{\text {NOTrs }}
$$

Le non-contrôlé est réalisé à l'aide de l'ensemble complet (1.47) avec la séquence déjà vu en (1.18). Cette opération demande donc sept portes élémentaires pour sa réalisation. De ce fait, une seule permutation requiert 21 opérations élémentaires (i.e., 'pas de temps', time steps) de sorte que $42 \times(|j-k|-1)$ opérations élémentaires sont requises pour réaliser le circuit de la figure 1.7 a). Ainsi, un grand nombre d'opérations sont nécessaires simplement pour distribuer l'information dans le registre. Ceci réduit le temps de calcul utile de l'ordinateur.

Il est heureusement possible de réduire ce nombre de façon significative. Premièrement, en prenant avantage des relations de commutation entre les portes élémentaires (1.47) et de la structure de la séquence (1.18) pour le $C_{\text {NOT }}$, il est possible de 'compiler' la séquence (1.50) en se débarrassant des portes redondantes. On obtient ainsi la séquence suivante pour le SWAP qui n'utilise 'que' 15 opérations élémentaires

$$
\begin{aligned}
\mathrm{S}_{\mathrm{Wrs}}= & e^{-i \pi / 4} \mathrm{R}_{\mathrm{xs}}(\pi / 2) \mathrm{C}_{\mathrm{Prs}}(-\pi / 2) \mathrm{R}_{\mathrm{zs}}(\pi / 2) \mathrm{R}_{\mathrm{xs}}(\pi / 2)\left[\mathrm{R}_{\mathrm{zs}}(\pi / 2) \mathrm{R}_{\mathrm{xr}}(\pi / 2)\right] \\
& \mathrm{C}_{\mathrm{Prs}}(-\pi / 2) \mathrm{R}_{\mathrm{zs}}(\pi / 2)\left[\mathrm{R}_{\mathrm{xr}}(\pi / 2) \mathrm{R}_{\mathrm{xs}}(\pi / 2)\right] \mathrm{C}_{\mathrm{Prs}}(-\pi / 2) \mathrm{R}_{\mathrm{zs}}(\pi / 2) \\
& \mathrm{R}_{\mathrm{xs}}(\pi / 2)\left[\mathrm{R}_{\mathrm{zs}}(\pi / 2) \mathrm{R}_{\mathrm{zr}}(\pi / 2)\right]
\end{aligned}
$$




\subsection{OPTIMISATION D'ALGORITHMES QUANTIQUES}

En prenant maintenant avantage de la possibilité de réaliser les opérations sur des qubits différents simultanément, il est possible de réduire davantage le nombre de 'pas de temps' utilisé à distribuer l'information dans le registre. En effet, puisque les opérations sur qubits distincts commutent, il est possible d'appliquer les portes entre crochets dans la séquence (1.51) simultanément. De plus, les permutations simultanées de la figure $1.7 \mathrm{~b}$ ) ne requièrent que $2\lceil|j-k| / 2\rceil$ pas de temps, où $\lceil x\rceil$ est le plus petit entier plus grand que $x$. Ceci est beaucoup plus efficace que la réalisation simpliste de la figure 1.7 a). Lorsque l'on réalise qu'il n'y a aucune raison de replacer les qubits à leur emplacement initial, il est possible de faire intervenir une dernière simplification. Une fois que les états d'une paire de qubits ont été rassemblés et ont interagi, la prochaine réorganisation devrait être faite de façon à optimiser l'exécution des opérations logiques suivantes.

En utilisant ces résultats, le nombre de pas de temps est réduit de $42(|j-k|-1)$ à $12\lceil|j-k| / 2\rceil$ pour une séquence unique de permutation. Pour les circuits impliquant plusieurs opérations contrôlées, d'autres améliorations sont possibles en réorganisant les qubits correctement pendant le calcul.

\subsubsection{Circuits optimisés}

Les optimisations décrites ci-haut sont indépendantes de l'algorithme à réaliser. Une observation spécifique à la QFT est

$$
\begin{aligned}
{\left[\mathrm{A}_{1}, \mathrm{~B}_{\mathrm{jk}}\right] } & =0 & \text { si } & l \neq j, k ; \\
{\left[\mathrm{B}_{\mathrm{jk}}, \mathrm{B}_{\mathrm{rs}}\right] } & =0 & \forall & j, k, r, s .
\end{aligned}
$$

La première de ces relations exprime simplement le fait que les portes agissant sur différents qubits commutent tandis que la seconde est vérifiée parce que les $B_{\mathrm{jk}}$ sont diagonaux dans la base de calcul. En respectant ces relations de commutation, il est donc possible de modifier l'ordre de la séquence afin d'appliquer les opérations lorsque cela est le plus favorable.

Afin de mettre en pratique ces concepts simples au profit de l'optimisation de la QFT, un programme C++ a été creé. Celui-ci prend en entrée la séquence originale pour la QFT, (1.37) dans le cas de 5 qubits, et lui ajoute des opérations de permutation selon la prescription de la figure $1.7 \mathrm{~b}$ ). Afin de minimiser la 'profondeur' 


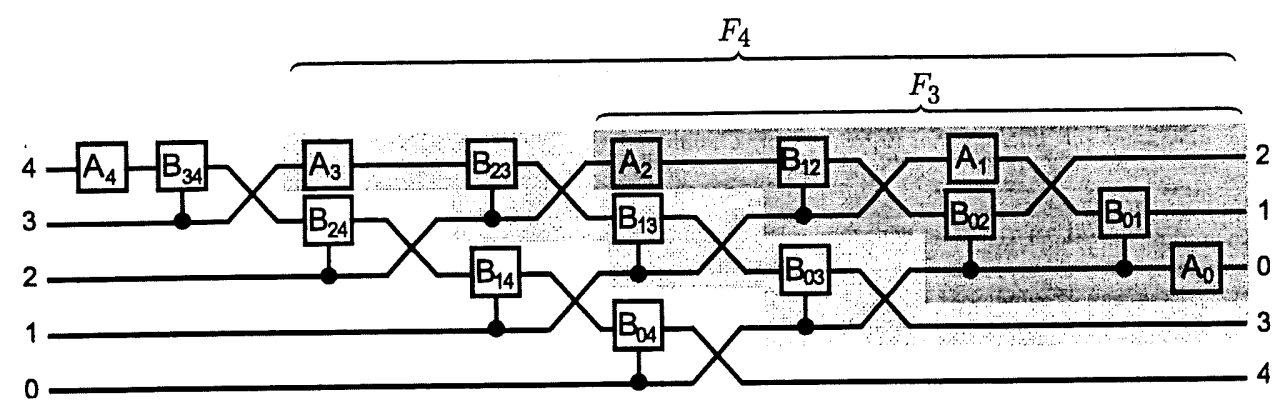

FIG. 1.8: Circuit réalisant la transformée de Fourier quantique d'un registre de 5 bits en tirant profit du parallélisme et en optimisant l'utilisation des permutations. Les nombres à gauche et à droite sont utilisés afin de suivre la progression de la position des différents états logiques. Le sous-circuit en gris foncé correspond à $F_{3}$.

(longueur) du circuit quantique, le programme maximise le parallélisme en regroupant les opérations qui commutent. Le résultat, toujours dans le cas de 5 qubits, est présenté à la figure 1.8 .

Si l'on suppose premièrement que toutes les opérations élémentaires prennent un temps $t_{\text {él }}$ pour être réalisées, le temps total nécessaire pour compléter un circuit de profondeur $p$ serait $t_{\hat{e} \mathbf{l}} \times p$. On exprime ici la profondeur à l'aide du nombre d'opérations élémentaires (1.47) requises pour réaliser les portes logiques (1.49) et (1.51). L'opération $B_{j k}$ est effectuée en deux pas de temps puisque toutes les opérations réalisant cette porte commutent et il est possible d'appliquer les deux premières simultanément. L'opération $A_{j}$ est quant à elle réalisée en trois pas de temps. Ainsi, le circuit de la figure 1.8 est réalisé en 95 pas de temps ${ }^{1}$. Sans optimisation, $F_{5}$ nécessite 20 permutations pour un total de 275 pas de temps.

La figure 1.9 compare, sur une échelle logarithmique, le coût en temps des circuits optimisés (cercles noirs) avec celui des circuits non-optimisés (carrés vides). Les circuits non-optimisés ne tirent pas profit du parallélisme et utilisent la stratégie de la Figure 1.7 a) pour les permutations. Ces circuits ont un coût en temps de $10 n-11 n^{2}+4 n^{3} \approx O\left(n^{3}\right)$, où $n$ est le nombre de qubits sur lesquels la transformée de Fourier est effectuée.

\footnotetext{
${ }^{1}$ Pour l'évaluation du coût en temps d'un circuit, lorsque deux opérations sont réalisées simultanément, seul le nombre de pas de temps de l'opération la plus coûteuse est retenue.
} 


\subsection{OPTIMISATION D'ALGORITHMES QUANTIQUES}

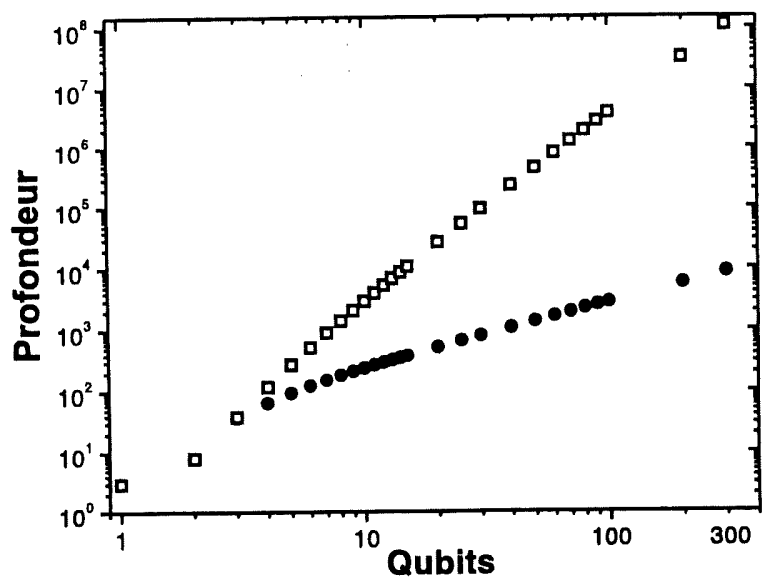

FIG. 1.9: Nombre de pas de temps pour réaliser la $Q F T$ en fonction du nombre de qubits, sur une échelle logarithmique. Les cercles noirs sont pour les circuits optimisés et les carrés vides pour les non-optimisés.

Afin d'aller au-delà des résultats numériques, il est utile de remarquer que le souscircuit en gris foncé du côté droit de la figure 1.8 correspond à une QFT optimisée sur trois bits. De plus, en ajoutant à cette transformation les quatre opérations logiques et les trois permutations qui sont dans la région en gris pâle de cette même figure, on obtient une QFT optimisée sur quatre bits. Ces opérations additionnelles ajoutent 29 pas de temps à $F_{3}{ }^{2}$. La même observation peut être faite en passant de $F_{4}$ à $F_{5}$ en ajoutant cinq opérations logiques et les 4 permutations qui sont à la gauche de la région en gris pâle, ajoutant à nouveau 29 pas de temps. En utilisant cette construction de manière récursive, il est possible d'obtenir le circuit optimisé pour $F_{n}$ lorsque l'on connaît le circuit optimisé pour $F_{n-1}$. Comme on le voit de l'exemple précédent, ceci se fait facilement en ajoutant $n$ opérations logiques et $n-1$ permutations à $F_{n-1}$ (pour $n>2$ ). Ces $O(n)$ opérations additionnelles ajoutent 29 pas de temps à $\mathrm{F}_{n-1}$. On obtient ainsi que le nombre de pas de temps pour réaliser $F_{n}$ est $8+29(n-2)$ pour $n>2$. On a donc obtenu pour la QFT des circuits de profondeur linéaire en $n$.

Ainsi, en tenant compte de la portée limitée de l'interaction qubit-qubit, il a été

\footnotetext{
${ }^{2}$ Les portes logiques $A_{3}, B_{23}$ et les première et seconde permutations de la région en gris pâle ne sont pas effectuées en parallèle avec des portes de $F_{3}$. Ces opérations additionnelles nécessite 29 pas de temps pour être exécutées.
} 


\subsection{OPTIMISATION D'ALGORITHMES QUANTIQUES}

possible d'obtenir un gain quadratique en $n$ par rapport aux circuits non-optimisés. L'utilisation efficace des permutations et du parallélisme (classique) est responsable de cette amélioration. En effet, un facteur de $O(n)$ provient du fait que $O\left(n^{3}\right)$ permutations sont nécessaires dans le cas non-optimisé tandis que le cas optimisé ne demande que $O\left(n^{2}\right)$ permutations. Un second facteur de $O(n)$ provient du fait que jusqu'à $n$ opérations peuvent simultanément être réalisées sur $n$ qubits. Comme dans le cas du calcul classique parallèle, ceci donne une accélération de $O(n)$.

Il est intéressant de remarquer que des circuits ayant une profondeur linéaire ont été obtenus à la référence [68] mais dans le cas plus simple où aucune limitation dans la portée de l'interaction entre qubits n'a été prise en considération ${ }^{3}$. En fait, une profondeur de $O(n)$ est le mieux que l'on puisse obtenir pour la QFT exacte donnée par la séquence (1.37). En effet, $n$ opérations doivent être appliquées sur le $n^{\text {ième }}$ qubit (ligne du haut dans la figure 1.5) et aucune opération agissant sur un même qubit ne peut être parallélisée. On en conclut donc que les limitations imposées par un design unidimensionnel restreint à des interactions au plus proche voisin ne semblent pas importantes en autant que le parallélisme soit possible et ce, tout au moins pour la QFT. Notons qu'un circuit similaire à celui de la figure 1.8 a été publié dans un autre contexte [75].

\subsubsection{Optimisation par recuit simulé}

Il est possible de diminuer davantage, par un facteur constant, la profondeur des circuits de QFT en commençant la procédure d'optimisation avec une permutation [respectant les règles de commutation (1.52)] de la séquence originale (1.37). On cherche alors la permutation minimisant la profondeur. Il s'agit d'un problème d'optimisation contrainte qui a beaucoup de similitudes avec la question du placement, importante en intégration de circuits (VLSI). Le placement consiste à arranger les

\footnotetext{
${ }^{3} \mathrm{Si}$ on calcule la profondeur en fonction du nombre d'opérations (et non selon le nombre de pas de temps requis pour réaliser ces opérations), les circuits obtenus ici ont une profondeur de $4(n-3)+7$ (pour $n>2$ ) et ceux de la référence [68] une profondeur de $2 n-1$. Les circuits obtenus ici ont donc une profondeur seulement deux fois plus grande (asymptotiquement) que les circuits obtenus dans le cas moins réaliste physiquement où les interactions ont une portée arbitraire et où le parallélisme est pris en considération.
} 


\subsection{OPTIMISATION D'ALGORITHMES QUANTIQUES}

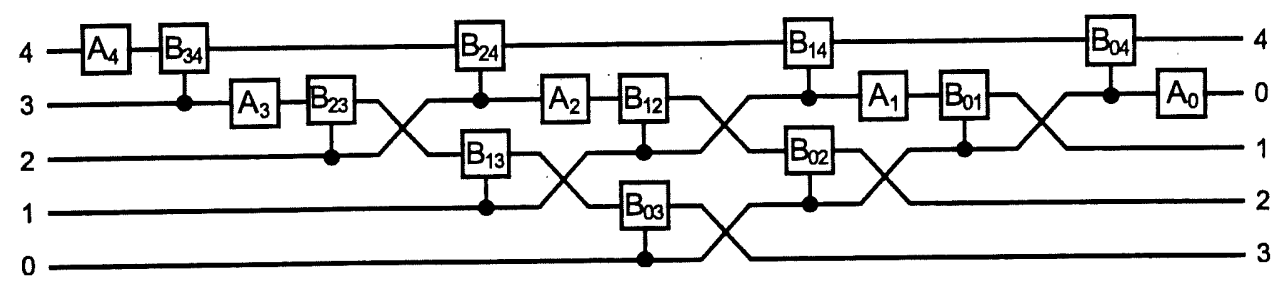

FIG. 1.10: Circuit amélioré par recuit simulé pour la QFT sur cinq qubits.

composantes du circuit (classique) de façon à minimiser la longueur des fils d'interconnexion et l'aire totale occupée par le circuit [76]. Il est connu que les heuristiques, comme le recuit simulé [77] ou la recherche tabou, donnent de bons résultats [76].

Le problème qui nous intéresse ici est semblable mais avec la complication additionnelle que réordonner deux opérations en un point quelconque du circuit change la séquence et, potentiellement, le nombre de permutations requises dans le reste du circuit. En se basant sur cette analogie, un algorithme de recherche par recuit simulé obtenant des circuits améliorés a été développé. Puisque le recuit simulé est une heuristique, nous n'obtiendrons pas nécessairement la solution optimale mais une solution s'en approchant. Ceci ne cause pas de problèmes puisque nous nous satisferons de toute amélioration par rapport aux circuits non optimisés.

Les circuits obtenus avec cette approche ne sont que quelques pour-cent plus courts que les meilleurs circuits (cercles noirs) de la figure 1.9. De plus, la recherche par recuit simulé est exigeante en temps de calcul et devient peu pratique pour seulement quelques dizaines de qubits. Cette amélioration supplémentaire sera néanmoins bienvenue pour les prototypes d'ordinateurs quantiques. Le circuit présenté à la figure 1.8 a été obtenu à l'aide de cet algorithme (une modification 'à la main' a été faite ici au circuit obtenu numériquement). Ce circuit ne requiert que 6 permutations et 83 pas de temps.

\subsubsection{Remarques et sommaire}

Finalement, il est important de mentionner que pour une QFT sur un grand nombre de qubits, l'application des opérations $\mathrm{B}_{\mathrm{jk}}$ pour $|j-k|$ grand est difficile expérimentalement. Cette difficulté vient du fait que les phases correspondantes seront très petites requérant ainsi l'habilité d'appliquer l'opération sur de très courts 
temps. Il est toutefois possible d'omettre les $B_{\mathrm{jk}}$ tels que $\pi / 2^{k-j}<\pi / 2^{m}$ pour un $m$ choisi et obtenir un résultat qui ne diffère que par un facteur multiplicatif $e^{i \varepsilon}$, avec $|\varepsilon| \leq 2 \pi N / 2^{m}$; du résultat de la QFT exacte [60]. En combinant ce résultat avec les techniques d'optimisation présentées ici, nous obtenons des circuits encore plus efficaces mais au prix d'une perte de précision. Ceci a été exploré par Cleve et Watrous [69]. Ceux-ci ont obtenu des circuits de profondeur $O(\log n)$ en supposant toutefois une portée arbitrairement grande pour l'interaction qubit-qubit. Notons que pour une architecture 1D, la limitation de l'interaction au premier voisin ne permet pas d'obtenir des profondeurs logarithmiques pour la QFT approximative. Pour obtenir un tel résultat, il est nécessaire d'avoir un couplage entre les qubits $i$ et $i+n / 2$ pour un registre linéaire de $n$ qubits.

Deux conclusions principales peuvent être tirées de cette procédure d'optimisation. 1) Les algorithmes quantiques peuvent êtres optimisés grâce au calcul classique. Les circuits obtenus demandent pour leur exécution des temps de cohérence beaucoup plus courts. 2) En autant que l'on permette les opérations en parallèle sur les qubits distincts, les ordinateurs quantiques peuvent être conçus avec une interaction qubit-qubit simple et ce, sans dégradation de leur performance. Cette conclusion ne s'applique toutefois pas au circuit de profondeur logarithmique mentionné ci-haut. Le parallélisme classique semble donc être une caractéristique souhaitable dans le design d'un ordinateur quantique. Ces conclusions sont importantes dans le contexte du design et de l'utilisation de prototypes d'ordinateurs quantiques.

Mentionnons finalement que les concepts présentés dans cette section sont applicables à tous les algorithmes quantiques. Plus particulierement le code C++ utilisé ici peut facilement être adapté à d'autres circuits. De même, la généralisation à d'autres architectures d'ordinateurs quantiques est simple. Il serait aussi intéressant de considérer d'autres types de géométrie de registre quantique $(2 \mathrm{D}$, quasi-1D,...) et le cas où l'interaction qubit-qubit n'est pas limitée au plus proche voisin mais a néanmoins une portée limitée [46,78]. En dernier lieu, les résultats obtenus ici pourront certainement être améliorés en travaillant directement avec les opérations élémentaires réalisant les différentes opérations logiques plutôt qu'avec les opérations logiques ellesmêmes. Comme on le constate en comparant les séquences (1.50) et (1.51), ceci peut certainement apporter des améliorations supplémentaires. Les résultats de cette section ont été publiés dans Phys. Rev. A [67]. 


\section{Chapitre 2}

\section{Calcul quantique géométrique}

Les critères décrits à la section $\S 1.2$, imposent aux ordinateurs quantiques d'avoir un long temps de cohérence et un faible taux d'erreurs. Heureusement, comme il a été décrit à la section $\S 1.5$, il est possible d'utiliser des codes afin de faciliter l'atteinte de ces objectifs. Une alternative à l'encodage est connue sous le nom de contrôle bangbang [79]. Plus de détails concernant cette approche et son application aux qubits supraconducteurs sont donnés à l'annexe C et à la référence [67].

Une autre approche ayant pour but de minimiser l'effet des imperfections est l'utilisation de la phase géométrique et, en particulier, de la phase géométrique adiabatique (ou phase de Berry) [80]. Dans le chapitre précédent, nous nous sommes souciés seulement de la phase dynamique. Par exemple, à la relation (1.9) décrivant une rotation $\mathrm{du}$ vecteur d'état sur la sphère de Bloch, la phase $\theta$ ne dépend que de l'énergie et du temps : $\theta=B_{i} t$ (avec $\hbar=1$ ). Ici, $t$ est le temps pendant lequel l'hamiltonien $B_{i} \sigma_{i}$ est non nul.

Contrairement à la phase dynamique, la phase de Berry ne dépend pas du temps et de l'énergie. Celle-ci est plutôt fonction de l'aire soutenue par le vecteur des paramètres de l'hamiltonien $\boldsymbol{B}(t)=\left\{B_{x}(t), B_{y}(t), B_{z}(t)\right\}$ lors d'un parcours fermé dans l'espace de ces paramètres. Cette phase est donc purement géométrique et ne dépend pas des détails de l'évolution sur le parcours. En autant que l'aire soit laissée inchangée, les imperfections sur le parcours laissent la phase géométrique invariante. Cette tolérance aux imperfections préservant l'aire a suggéré à certains auteurs que la phase géométrique pourrait être utile pour la réalisation d'opérations logiques quan- 
tiques intrinsèquement tolérantes aux imperfections. Par exemple, il a été suggéré que la phase de Berry soit insensible au bruit sur le parcours dans l'espace des paramètres [81]. Jusqu'à maintenant, il y a eu plusieurs suggestions concernant l'utilisation de la phase de Berry dans le calcul quantique avec différents systèmes physiques [81-84]. L'application de la phase géométrique adiabatique non-abélienne [85] au calcul quantique a aussi été le sujet de plusieurs publications [86-89].

Nous nous intéresserons ici à l'application d'un autre type de phase géométrique au calcul quantique : la phase géométrique non-adiabatique ou encore de AharonovAnandan (AA) [90]. Comme dans le cas de la phase de Berry, cette dernière est purement géométrique. Elle est reliée à l'aire soutenue par le vecteur d'état dans l'espace projectif (défini plus loin) durant une évolution cyclique. On peut donc s'attendre à ce que les portes quantiques basées sur cette phase aient une certaine tolérance intrinsèque aux imperfections.

Dans ce chapitre, on montre que, par rapport à la phase de Berry, la phase AA semble avoir plusieurs avantages pour le calcul quantique. On discute aussi de l'observation de cette phase sur un qubit à l'aide d'un second qubit. L'observation de cette phase avec un qubit supraconducteur de charge est aussi abordée. L'application à d'autres architectures d'ordinateurs quantiques constitue une généralisation simple. Le but principal de ce chapitre toutefois est de montrer que les arguments exposés ci-haut concernant la tolérance au bruit ne tiennent pas. En fait, on montre que les opérations logiques basées sur la phase AA (appelées ensuite portes de phase AA ou, plus simplement, portes $\mathrm{AA}$ ) sont plus affectées par le bruit dans les paramètres de contrôle de l'hamiltonien que les portes dynamiques équivalentes. On en conclut donc que les opérations logiques basées sur la phase AA ne sont pas utiles en pratique pour le calcul. Ce résultat est confirmé numériquement pour différents types de bruits. De plus, en utilisant les mêmes approches analytique et numérique, on montre que cette conclusion s'applique aussi pour les portes logiques basées sur la phase de Berry.

Alors que la majeure partie de ce travail avait été complétée, des idées similaires ont été publiées par Xiang-Bin et Keiji [91,92]. Ces auteurs n'abordent toutefois pas la sensibilité des portes AA aux imperfections. Depuis, des idées similaires ont été publiées par différents auteurs et pour différents systèmes physiques [93-97]. Dans la plupart des cas, ces publications ne présentent que des généralisations simples des 
idées discutées ici. De plus, la sensibilité aux imperfections n'a pas été abordée dans ces publications plus récentes. Ce chapitre est basé sur les références [98] et [99].

\subsection{Portes géométriques adiabatiques vs non-adiabatiques}

Dans cette section, les concepts principaux concernant la phase de Berry et ses inconvénients dans le contexte du calcul quantique sont exposés. La phase AA est ensuite introduite, ainsi que son application au calcul quantique. Le lecteur intéressé trouvera des détails supplémentaires concernant les phases géométriques en général dans différents articles de revues $[100,101]$ et livre [102].

\subsubsection{Phase de Berry et calcul géométrique}

Considérons un système physique dont l'hamiltonien $H(t)$ est contrôlé par un ensemble de paramètres extérieurs $\boldsymbol{B}(t)$. Sous variations adiabatiques de $\boldsymbol{B}(t)$, si le système est initialement dans un état propre de $H(0)$, il restera un état propre $\left|\psi_{n}(t)\right\rangle=\left|\psi_{n}(\boldsymbol{B}(t))\right\rangle$ de l'hamiltonien instantané $H(t)$. De plus, si le spectre de $H$ n'est pas dégénéré sur un parcours fermé $C$ dans l'espace des paramètres et si ce parcours est choisi de telle sorte que $\boldsymbol{B}(0)=\boldsymbol{B}(\tau)$, alors suite à l'évolution sur $C$, l'état au temps $t=\tau$ ne différera de l'état au temps $t=0$ que par un facteur de phase. Berry a montré que cette phase peut avoir une composante dynamique et une composante géométrique. Cette dernière ne dépend que de $C$ [80] :

$$
\gamma_{B}(C)=i \oint_{C}\left\langle\phi_{n}(\boldsymbol{B})\left|\nabla_{\boldsymbol{B}}\right| \phi_{n}(\boldsymbol{B})\right\rangle \cdot d \boldsymbol{B}
$$

où $\nabla_{B}$ est le gradient par rapport aux paramètres $B$. Pour un qubit, cette relation se réduit à plus ou moins la moitié de l'angle solide soutenu par $C$.

Si l'état initial est une superposition des états propres $\left|\psi_{n}\right\rangle$ de l'hamiltonien, alors chacun de ceux-ci acquerra une phase $\phi_{n}:\left|\psi_{n}(\tau)\right\rangle=\mathrm{U}(\tau)\left|\psi_{n}(0)\right\rangle=e^{i \phi_{n}}\left|\psi_{n}(0)\right\rangle[103]$. Ces phases auront généralement une contribution dynamique et une contribution géométrique. Pour un tel état initial, l'évolution n'est pas cyclique. Cela ne cause toutefois pas d'ambiguité puisque la phase de Berry est définie sur l'espace des paramètres. Dans le langage du calcul quantique, l'évolution décrite ci-haut correspond 


\subsection{PORTES GÉOMÉTRIQUES ADIABATIQUES VS NON-ADIABATIQUES}

à une opération $R_{z}(\theta)$ sur un bit (lorsque $H$ est l'hamiltonien d'un qubit seulement) mais où $\theta$ a une origine géométrique. Un exemple de cette opération sur un qubit supraconducteur de charge est décrit à l'annexe $\mathrm{A}$.

Il ressort de la discussion précédente que l'application de la phase géométrique adiabatique au calcul quantique a plusieurs inconvénients. Premièrement, les ordinateurs quantiques auront fort probablement un court temps de cohérence. Afin de tirer profit au maximum de ce temps, les opérations logiques devraient être exécutées rapidement. La contrainte d'adiabaticité signifie toutefois que les portes basées sur la phase de Berry seront lentes, réduisant ainsi le facteur de qualité effectif de l'ordinateur quantique. Le facteur de qualité est le ratio du temps de décohérence sur le temps requis pour compléter une opération logique typique, $Q \equiv t_{\mathrm{dec}} / t_{\mathrm{op}}$.

Un autre désavantage de la porte géométrique adiabatique est que, au cours de l'évolution adiabatique, une composante géométrique et une composante dynamique sont acquises. Cette dernière n'est pas tolérante au bruit préservant l'aire et doit être annulée. Il est possible de réaliser cette annulation en utilisant la refocalisation, une technique basée sur l'écho de spins en RMN. Cette technique demande de répéter l'évolution adiabatique à deux reprises [81-83]. En effet, imaginons que l'état initial soit $|\psi\rangle=a|0\rangle+b|1\rangle$. Après l'évolution adiabatique sur $C$, le résultat est

$$
\left|\psi_{C}\right\rangle=a e^{i\left(\delta_{0}+\gamma_{B}\right)}|0\rangle+b e^{i\left(\delta_{1}-\gamma_{B}\right)}|1\rangle,
$$

où $\delta_{i}$ est la phase dynamique pour l'état logique $|i\rangle$ et $\gamma_{B}$ est la phase géométrique correspondant à $C$. Afin d'annuler la composante dynamique, on applique d'abord une impulsion $\pi$ rapide échangeant les valeurs binaires

$$
\left|\psi_{C \pi}\right\rangle=a e^{i\left(\delta_{0}+\gamma_{B}\right)}|1\rangle+b e^{i\left(\delta_{1}-\gamma_{B}\right)}|0\rangle .
$$

Maintenant, on fait ensuite parcourir au système la boucle $C$ mais dans la direction inverse (notée $\tilde{C}$ ). La phase dynamique sur $\tilde{C}$ est encore $\delta_{i}$ tandis que la phase géométrique a le signe inverse par rapport à l'évolution sur $C \cdot[80]$

$$
\left|\psi_{C \pi \bar{C}}\right\rangle=a e^{i\left(\delta_{0}+\delta_{1}+2 \gamma_{B}\right)}|1\rangle+b e^{i\left(\delta_{1}+\delta_{0}-2 \gamma_{B}\right)}|0\rangle .
$$

Finalement, une seconde impulsion $\pi$ est appliquée afin de restaurer les valeurs binaires initiales

$$
\left|\psi_{C \pi \tilde{C} \pi}\right\rangle=a e^{i\left(\delta_{0}+\delta_{1}+2 \gamma_{B}\right)}|0\rangle+b e^{i\left(\delta_{1}+\delta_{0}-2 \gamma_{B}\right)}|1\rangle .
$$




\subsection{PORTES GÉOMÉTRIQUES ADIABATIQUES VS NON-ADIABATIQUES}

À un facteur de phase globale près, cette séquence d'évolution donne une phase relative purement géométrique

$$
\left|\psi_{C \pi \tilde{C} \pi}\right\rangle=e^{i\left(\delta_{0}+\delta_{1}+2 \gamma_{B}\right)}\left(a|0\rangle+b e^{-4 i \gamma_{B}}|1\rangle\right) .
$$

Quoique cette séquence de manipulations donne le résultat voulu, l'annulation de la phase dynamique augmente le temps requis pour réaliser l'opération de phase $R_{z}$. De plus, avec des manipulations imparfaites, la phase dynamique ne s'annulera pas complètement introduisant des erreurs.

Une troisième difficulté est que la phase géométrique adiabatique n'est possible que si des parcours non triviaux dans l'espace des paramètres sont possibles. En d'autres mots, l'hamiltonien à un qubit doit être de la forme

$$
H=\frac{1}{2} B_{x}(t) \sigma_{x}+\frac{1}{2} B_{y}(t) \sigma_{y}+\frac{1}{2} B_{z}(t) \sigma_{z},
$$

où le contrôle de tous les champs $B_{i}(t)$ est possible. Un tel contrôle n'est pas possible pour plusieurs des suggestions actuelles d'ordinateurs quantiques. Le contrôle de deux champs, $B_{x}$ et $B_{z}$ par exemple, est plus standard. Dans ce cas, les parcours dans l'espace des paramètres sont limités au plan $x-z$ et la phase de Berry (relative) est limitée à des multiples entiers de $2 \pi$, ce qui n'est d'aucun intérêt pour le calcul quantique. Notons que le contrôle de trois champs est possible en RMN où la phase de Berry a été observée expérimentalement [81]. Plus récemment, Falci et al. [83] ont suggéré une modification au design original de qubits supraconducteurs de charge permettant un $B_{y}$ non nul et donc des parcours non triviaux. Voir l'annexe A pour plus de détails. Notons aussi que certains designs récents de qubits supraconducteurs permettent le contrôle de $B_{y}$. Ces designs récents seront décrits à la section §3.4.3.

Cette nécessité d'avoir un contrôle sur plusieurs termes de l'hamiltonien à un qubit implique souvent des contraintes, difficultés expérimentales et sources de bruit et de décohérence additionnelles. Ceci est clairement contradictoire avec les efforts de simplification des designs d'ordinateurs quantiques basés sur l'universalité encodée. Ces efforts sont décrits brièvement à la section $\S 1.5$.

Comme nous le verrons, les problèmes mentionnés ici, à savoir l'évolution lente, la nécessité d'annulation de la phase dynamique et le contrôle sur plusieurs champs effectifs, semblent être résolus lorsque l'on considère la généralisation non adiabatique de la phase de Berry : la phase de Aharonov-Anandan (AA). 


\subsection{PORTES GÉOMÉTRIQUES ADIABATIQUES VS NON-ADIABATIQUES}

\subsubsection{Phase géométrique non-adiabatique}

La phase géométrique non adiabatique est introduite en se restreignant, pour une évolution donnée, aux états initiaux satisfaisants

$$
|\psi(\tau)\rangle=U(\tau)|\psi(0)\rangle=e^{i \phi}|\psi(0)\rangle .
$$

Pour les évolutions non adiabatiques, ces états initiaux cycliques [104] ne sont généralement pas des états propres de l'hamiltonien du système mais de l'opérateur d'évolution. Aharonov et Anandan [90] ont montré que la phase totale acquise par ces états sur l'intervalle $[0, \tau]$, sur lequel ils sont cycliques, est la somme d'une contribution dynamique $(\hbar=1)$,

$$
\delta=-\int_{0}^{\tau} d t\langle\psi(t)|H(t)| \psi(t)\rangle
$$

et d'une contribution géométrique,

$$
\beta=\phi-\delta
$$

Cette dernière est la phase $\mathrm{AA}$. Ce résultat est exact, il ne repose pas sur une approximation adiabatique mais est restreint aux états initiaux cycliques pour lesquels l'équation (2.8) s'applique.

Malgré que (2.10) soit une définition opérationnelle pratique de la phase AA, il est simple d'obtenir une expression plus explicite [90]. Considérons l'état $|\tilde{\psi}(t)\rangle$ défini de sorte que $|\tilde{\psi}(t)\rangle=e^{i f(t)}|\psi(t)\rangle$ avec $f(\tau)-f(0)=\phi$. Avec cette définition, $|\tilde{\psi}(\tau)\rangle=|\psi(0)\rangle$ et, de l'équation de Schrödinger, on obtient aisément

$$
-\frac{d f}{d t}=\frac{1}{\hbar}\langle\psi(t)|H| \psi(t)\rangle-\left\langle\tilde{\psi}\left|i \frac{d}{d t}\right| \tilde{\psi}\right\rangle .
$$

En intégrant cette relation, le premier terme du membre de droite donne l'équation (2.9) et le second terme est une expression alternative pour la phase AA

$$
\beta=i \int_{0}^{\tau} d t\left\langle\tilde{\psi}(t)\left|\frac{d}{d t}\right| \tilde{\psi}(t)\right\rangle=i \oint_{C^{*}}\langle\tilde{\psi}|d| \tilde{\psi}\rangle
$$

La composante $\beta$ ne dépend que du parcours fermé $C^{*}$. Ce parcours, contrairement au cas de la phase de Berry, n'est pas défini dans l'espace des paramètres de l'hamiltonien mais plutôt dans l'espace projectif $\mathcal{P}$ [90]. Pour un (pseudo-)spin $1 / 2$, qui est le 


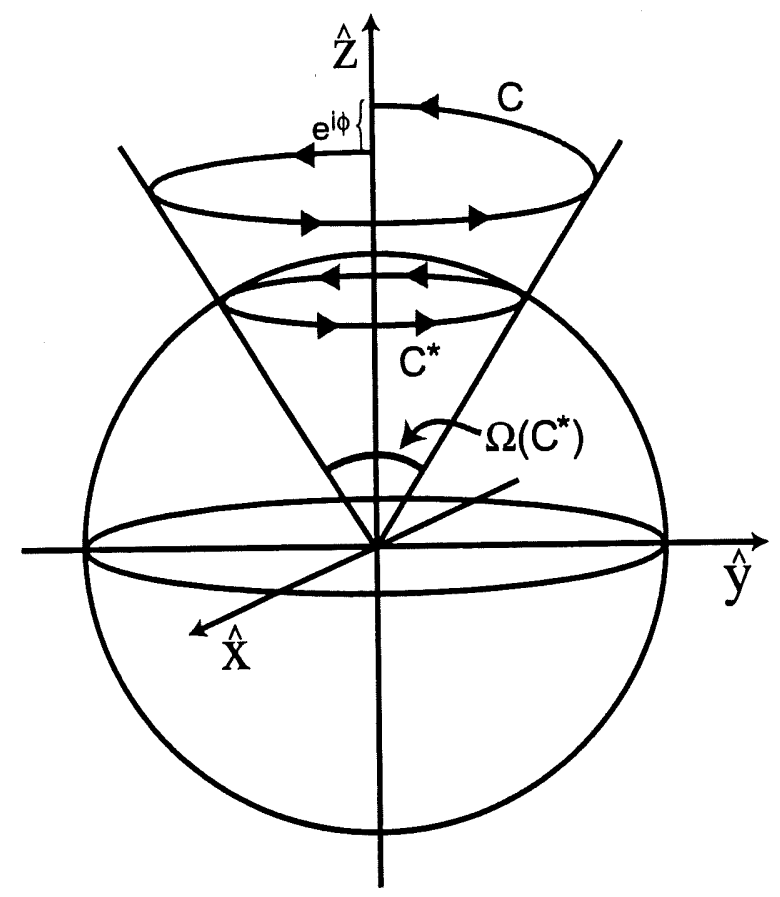

FIG. 2.1: Pour un système à deux niveaux, l'espace projectif $\mathcal{P}$ est isomorphe à la sphère de Bloch. Pour une évolution cyclique, un parcours ouvert $C$ dans l'espace d'Hilbert $\mathcal{H}$ est mappé sur un parcours fermé $C^{*}$ dans $\mathcal{P}$. La phase géométrique $\beta$ est $\pm \Omega\left(C^{*}\right) / 2$, où $\Omega\left(C^{*}\right)$ est l'angle solide soutenu par le vecteur de Bloch sur $C^{*}$. Figure adaptée de la Réf. [82].

système d'intérêt en calcul quantique, l'espace projectif est isomorphe à la sphère de Bloch (i.e. l'espace des matrices densités $2 \times 2$ ). Dans ce cas, $C^{*}$ est 'tracé' sur la surface de la sphère de Bloch par le vecteur de Bloch $\boldsymbol{b}(t)$. La phase géométrique non adiabatique $\beta$ est alors donnée par plus ou moins la moitié de l'angle solide soutenu par le vecteur de Bloch. La situation est décrite de façon schématique à la figure 2.1.

La phase $\beta$ est purement géométrique [101]. En effet, (i) elle ne dépend pas de la vitesse de progression sur $C^{*}$, contrairement à $\delta$. (ii) Elle est indépendante de l'hamiltonien générant l'évolution sur $C^{*}$, à nouveau contrairement à $\delta$. Finalement, (iii) cette phase est indépendante de l'état $|\tilde{\psi}(t)\rangle$ choisi. 


\subsection{PORTES GÉOMÉTRIQUES ADIABATIQUES VS NON-ADIABATIQUES}

\subsubsection{Calcul géométrique non-adiabatique}

Intéressons-nous maintenant à la phase $\mathrm{AA}$ en tant qu'outil pour le calcul quantique. Le premier des inconvénients de la phase de Berry est déjà réglé puisque la contrainte d'adiabaticité a été relâchée en choisissant des états initiaux cycliques appropriés.

Le second désavantage de la phase adiabatique est réglé en se restreignant à des évolutions satisfaisant

$$
\langle\psi(t)|H(t)| \psi(t)\rangle=0
$$

en tout temps. Pour de telles évolutions, la contribution dynamique (2.9) à la phase total est nulle et seulement une contribution géométrique est acquise sur $C^{*}$. Afin que (2.13) soit nulle, l'axe de rotation doit toujours être orthogonal au vecteur d'état. Les parcours correspondants sont alors des polygones sphériques où chaque segment se trouve le long d'un grand cercle sur la sphère de Bloch. Que de telles évolutions soient possibles est un avantage important de la phase AA. En effet, il n'est alors pas nécessaire d'annuler la phase dynamique par la technique d'écho de spins.

Pour traiter le troisième inconvénient, limitons notre attention aux hamiltoniens pour lesquels seulement deux champs de contrôle sont non nuls

$$
H=\frac{1}{2} B_{x}(t) \sigma_{x}+\frac{1}{2} B_{z}(t) \sigma_{z} .
$$

Si les champs selon $x$ et $z$ peuvent être simultanément non nuls, alors l'évolution suivante est possible

$$
\mathrm{R}_{\mathrm{z}}^{\mathrm{AA}}(\theta) \equiv \mathrm{R}_{\mathrm{x}}(\pi / 2) \mathrm{R}_{n}(\pi) \mathrm{R}_{\mathrm{x}}(\pi / 2),
$$

avec pour la seconde porte $n=(-\cos \theta, 0, \sin \theta)$ et $B_{n}=\sqrt{B_{x}^{2}+B_{z}^{2}}$. Cette opération a pour action $R_{z}^{A A}(\theta)|0\rangle=e^{-i \theta}|0\rangle$. La figure 2.2 a) présente le parcours correspondant sur la sphère de Bloch. Puisque cette évolution satisfait (2.13), la phase dynamique est nulle et la phase géométrique est donc simplement $-\theta$. En variant l'angle $\theta$ de l'axe de rotation, il est possible d'obtenir une phase géométrique arbitraire. Toutefois, pour les architectures où les champs $B_{x}$ et $B_{z}$ ne peuvent être non nuls simultanément, l'évolution est restreinte à $\boldsymbol{n}= \pm \boldsymbol{z}$ et donc à des multiples entiers de $\pi / 2$ pour $\theta$. Les détails de la réalisation de la séquence (2.15) à l'aide d'un qubit supraconducteur de charge sont présentés à l'annexe $\mathrm{A}$. 


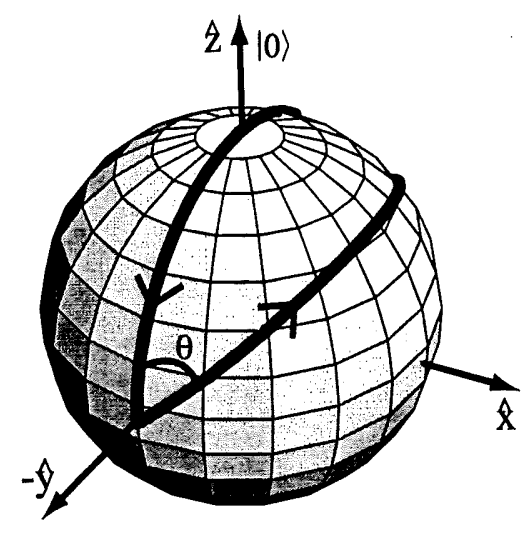

(a)

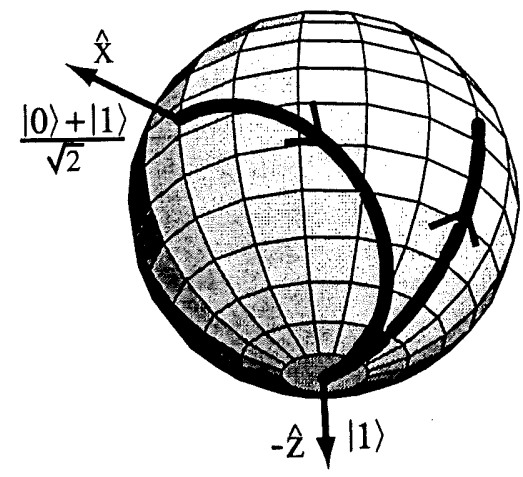

(b)

FIG. 2.2: a) Évolution du vecteur de Bloch sur la sphère de Bloch pour la séquence d'impulsions (2.15). L'état initial (cyclique) est $|0\rangle$. En utilisant plutôt $|1\rangle$, on obtient un parcours similaire mais centré au pôle sud de la sphère de Bloch. b) La séquence de rotation (2.15) appliquée à la superposition $(|0\rangle+|1\rangle) / \sqrt{2}$ ne produit pas un parcours fermé.

La séquence (2.15) sur la superposition $(a|0\rangle+b|1\rangle) / \sqrt{2}$ donne

$$
\frac{1}{\sqrt{2}}\left(a e^{-i \theta}|0\rangle+b e^{+i \theta}|1\rangle\right)
$$

où la différence de phase entre $|0\rangle$ et $|1\rangle$ est observable par interférence ${ }^{1}$. Toutefois, quoique l'état final dépende de la phase AA correspondant à l'évolution de $|0\rangle$ et |1) séparément, il ne s'agit plus d'une évolution cyclique lorsque l'on agit sur la superposition de ces états.

Pour la phase de Berry, une situation similaire ne cause pas d'ambiguïté. Dans ce cas, comme il a été mentionné à la section $\$ 2.1 .1$, si l'état initial est une superposition de vecteurs propres, le résultat final ne correspond pas non plus à une évolution cyclique de la superposition. Néanmoins, la phase acquise par chacun des états propres est de nature géométrique puisque le parcours est fermé dans l'espace des paramètres, peu importe ce qu'il en est de l'espace projectif [103].

\footnotetext{
${ }^{1}$ Suite, par exemple, à l'application d'une porte de Hadamard, la probabilité de mesurer chacun des états logiques dépend de $\theta: H\left(a e^{-i \theta}|0\rangle+b e^{+i \theta}|1\rangle\right) / \sqrt{2}=\left(\left[a e^{-i \theta}+b e^{+i \theta}\right]|0\rangle+\left[a e^{-i \theta}-\right.\right.$ $\left.\left.b e^{+i \theta}\right]|1\rangle\right) / 2$.
} 


\subsection{PORTES GÉOMÉTRIQUES ADIABATIQUES VS NON-ADIABATIQUES}

Dans le cas non-adiabatique toutefois, le parcours doit être fermé dans $\mathcal{P}$ et, comme on le voit à la figure $2.2 \mathrm{~b}$ ), cela n'est clairement pas le cas. Identifier la phase AA en utilisant la définition originale de Aharonov et Anandan est alors plus subtil. Une telle situation a suggéré à certains auteurs $[100,105]$ que la phase AA n'est en fait pas observable pour les évolutions cycliques sur un système quantique isolé. La raison est simplement que cette phase est définie uniquement pour les évolutions cycliques et que, puisque les phases globales ne sont pas physiques, les propriétés observables restent inchangées pour de telles évolutions.

Cette ambiguïté peut être levée en définissant une phase non-adiabatique nonabélienne [106]. Dans ce contexte, les facteurs de phase de la superposition (2.16) ont une nature géométrique. Malgré cette définition plus générale, il serait intéressant d'observer directement la phase AA. Une telle observation a été réalisée en RMN par Suter et al. [107]. Dans le langage du calcul quantique, l'analogue de cette expérience est d'utiliser un qubit auxiliaire afin d'observer la phase du qubit subissant l'évolution cyclique. Explicitement, on débute les manipulations avec le premier qubit (l'auxiliaire) dans une superposition d'états arbitraire

$$
(a|0\rangle+b|1\rangle)|0\rangle .
$$

Ensuite, on applique la séquence (2.15) sur le second qubit uniquement si le premier est dans l'état $|1\rangle$. Cette opération conditionnelle est réalisée à l'aide de

$$
\begin{aligned}
C_{\mathrm{R}_{\mathrm{z}}^{\mathrm{AA}}} & \equiv \mathrm{C}_{\mathrm{NOT}} \mathrm{R}_{\mathrm{z2}}^{\mathrm{AA}}(-\theta / 2) \mathrm{C}_{\mathrm{NOT}} \mathrm{R}_{\mathrm{z2}}^{\mathrm{AA}}(\theta / 2) \\
& =\left(\begin{array}{llll}
1 & & & \\
& 1 & & \\
& & e^{-i \theta} & \\
& & & e^{+i \theta}
\end{array}\right) .
\end{aligned}
$$

Ici, $\mathrm{R}_{\mathrm{z} 2}^{\mathrm{AA}}( \pm \theta / 2)$ est la séquence (2.15) appliquée sur le qubit \#2. Cette séquence d'opérations a pour résultat

$$
\begin{aligned}
\mathrm{C}_{\mathrm{R}_{2}^{\mathrm{AA}}}(a|00\rangle+b|10\rangle) & =a|00\rangle+b e^{-i \theta}|10\rangle \\
& =\left(a|0\rangle+e^{-i \theta} b|1\rangle\right)|0\rangle .
\end{aligned}
$$

Le résultat net est équivalent à une opération logique de phase (géométrique) sur le 
premier qubit. Cette phase peut être observée par interférence ${ }^{2}$. Dans cette situation, il n'y a aucune ambiguïté à définir la phase AA : le second qubit subit une évolution cyclique mais la phase reste néanmoins observable puisque l'évolution du système total n'est pas cyclique.

Notons que le $C_{\text {NOT }}$ peut être réalisé à l'aide de la séquence (1.18) basée sur l'opération $C_{p}$. Évidemment, cette réalisation particulière du non-contrôlé est spécifique aux architectures d'ordinateurs quantiques ayant $C_{P}$ dans leur répertoire. Des séquences alternatives pour le $\mathrm{C}_{\mathrm{NOT}}$ peuvent toutefois être trouvées pour les autres architectures. Pour les qubits de charge, l'interaction $\sigma_{z} \otimes \sigma_{z}$ à la base de l'opération $C_{p}$ peut être obtenue grâce à un couplage capacitif [83]. Cette interaction sera décrite en plus de détails au chapitre 4 .

Finalement, en utilisant (1.18) et (2.15), il est possible de 'compiler' la séquence $(2.18)$ de $2 \times(7+3)=20$ à 18 opérations élémentaires. De plus, on peut vérifier que la phase dynamique s'annule sur (2.18). Il s'agit donc d'une opération à deux qubits purement géométrique. Cette porte logique nécessite toutefois 18 opérations élémentaires pour sa réalisation, un nombre plutôt grand pour une porte ayant pour but de réaliser une opération de phase (géométrique) contrôlée résistante aux imperfections.

\subsection{Tolérance au bruit des paramètres de contrôle}

Afin que les portes logiques géométriques soient utiles au calcul quantique, elle doivent avoir une certaine tolérance naturelle aux fluctuations des paramètres de contrôle. Ces fluctuations introduisent des imperfections aux niveaux de l'angle et de l'axe de rotation des opérations élémentaires réalisant les portes géométriques. En retour, ces imperfections modifient l'évolution unitaire globale appliquée sur le qubit. La phase finale correspondante peut alors avoir une composante dynamique. Il est important de noter que la question ici n'est pas de savoir si les imperfections

\footnotetext{
${ }^{2}$ Ceci est équivalent, par exemple, à une expérience d'interférométrie neutronique où le premier qubit représente la position du neutron et le second son spin. Puisqu'un qubit habite, par construction, un espace d'Hilbert de dimensions deux, il est nécessaire d'utiliser plus d'un qubit pour simuler une expérience d'interférométrie utilisant plus d'un degré de liberté d'une particule.
} 


\subsection{TOLÉRANCE AU BRUIT DES PARAMÈTRES DE CONTRÔLE}

affectent la composante dynamique ou géométrique puisque tout facteur de phase non voulu représente une erreur dans le calcul quantique. Dans cette section, nous nous intéressons donc à l'erreur sur la phase totale due aux fluctuations des paramètres de contrôle autour des valeurs requises pour réaliser une opération purement géométrique dans le cas sans imperfections.

Considérons d'abord un cas simple : une erreur $\epsilon$ sur l'angle de la première opération élémentaire de la séquence (2.15) :

$$
\mathrm{R}_{\mathrm{x}}(\pi / 2) \mathrm{R}_{n}(\pi) \mathrm{R}_{\mathrm{x}}(\pi / 2+\epsilon) .
$$

On ne considère pas les portes additionnelles (2.18) pour le moment. Évidemment, il ne s'agit pas d'une erreur qui préserve l'aire et on n'est pas en droit de s'attendre à ce que la phase AA soit laissée inchangée. Toutefois, il s'agit du type d'erreurs qui aura lieu si le champ $B_{x}(t)$ fluctue.

En appliquant la séquence erronée (2.20) sur l'état $|0\rangle$, il est facile de vérifier que la porte de phase AA n'est pas tolérante à cette erreur :

$$
\cos (\epsilon / 2) e^{-i \theta}|0\rangle-i \sin (\epsilon / 2) e^{+i \theta}|1\rangle .
$$

L'évolution n'est plus cyclique et on ne peut plus définir la phase AA dans cette situation (tout au moins pas dans la base de calcul). De plus, même au premier ordre en $\epsilon$. l'évolution n'est pas cyclique. La porte de phase AA n'est donc pas tolérante aux petites imperfections. Celles-ci peuvent amener le vecteur d'état hors des grands cercles. Une phase dynamique s'accumule alors. Dans certains cas, comme ici, l'évolution n'est plus cyclique et la phase AA ne peut plus être définie dans la base de calcul.

Il est possible d'avoir une meilleure compréhension de l'effet du bruit sur la porte de phase $\mathrm{AA}$ et de comparer son comportement avec la porte de phase dynamique usuelle

$$
\mathrm{R}_{\mathbf{z}}(\theta)=e^{-i \theta \sigma_{z} / 2}
$$

en étudiant l'hamiltonien

$$
H=\frac{1}{2} \sum_{i=x, z}\left(B_{i}(t)+\delta B_{i}(t)\right) \sigma_{i} .
$$

Ici, $\delta B_{i}$ représente les fluctuations du paramètre de contrôle $B_{i}$. Les fluctuations de ces champs effectifs constituent probablement la source la plus importante de décohérence 


\subsection{TOLÉRANCE AU BRUIT DES PARAMÈTRES DE CONTRÔLE}

pour les qubits supraconducteurs [108]. Pour les qubits de charge, cela correspond au bruit Nyquist-Johnson du voltage de grille $V_{g}$ et du courant générant le flux extérieur $\Phi_{x}$. Plus de détails sur ces paramètres de contrôle seront présentés à la section §3.1.1.

Sans bruit, $R_{z}^{A A}(\theta / 2)$ et $R_{z}(\theta)$ ont le même effet global sur un vecteur d'état (les phases n'étant évidemment pas de même nature). Afin de comparer ces portes en présence de bruit, la propriété de composition de l'opérateur d'évolution est utilisée :

$$
\mathrm{U}(t)=\mathcal{T} e^{-i \int_{0}^{t} d t^{\prime} H\left(t^{\prime}\right)}=\lim _{N \rightarrow \infty} \prod_{n=1}^{N} \mathrm{U}(n),
$$

où $\mathrm{U}(n)=\exp (-i H(n) t / N)$ et $H(n)$ est l'hamiltonien pendant le $n^{\text {ième intervalle. }}$ On utilise ici les unités telles que $\hbar=1$. Afin de simuler le bruit, les champs $\delta B_{i}(n)$ sont pris comme variables aléatoires indépendantes tirées d'une distribution uniforme de probabilité dans l'intervalle $\pm \delta B_{\max }$. Sans bruit, la décomposition (2.24) est exacte pour toutes valeurs de $N$ puisque les opérations logiques $\mathrm{R}_{z}^{A A}(\theta / 2)$ et $\mathrm{R}_{\mathbf{z}}(\theta)$ sont réalisées à l'aide d'hamiltonien constant par morceaux. En présence de bruit, on suppose que les $\delta B_{i}$ sont indépendants du temps sur l'intervalle $\Delta t \equiv t / N_{i}$. On définit donc $\Delta t$ comme étant le temps de corrélation du bruit. Ce temps sera le même pendant l'application de toutes les opérations élémentaires $R_{i}$. Notons qu'avec la décomposition (2.24), l'évolution est explicitement unitaire.

Afin de comparer $R_{z}^{A A}(\theta / 2)$ et $R_{z}(\theta)$, on calcule la distance (trace distance) [1]

$$
D(\mathrm{U}, \mathrm{V})=\operatorname{Tr}\left\{\sqrt{(\mathrm{U}-\mathrm{V})^{\dagger}(\mathrm{U}-\mathrm{V})}\right\}
$$

des opérations géométrique et dynamique avec bruit par rapport à la porte $R_{z}(\theta)$ sans imperfection. En utilisant plutôt la 'fidélité moyenne' [109] pour comparer numériquement les opérations ayec et sans bruit les mêmes conclusions ont été obtenues. La distance $D(\mathrm{U}, \mathrm{V})$ prend des valeurs entre 0 et 4 , avec $D(\mathrm{U}, \mathrm{V})=0$ seulement si $\mathrm{U}$ et $\mathrm{V}$ sont identiques. Ainsi, si la porte AA doit être plus tolérante que sa contrepartie dynamique (et donc avantageuse pour le calcul) alors l'inégalité

$$
D\left(\tilde{\mathrm{R}}_{\mathbf{z}}^{\mathrm{AA}}(\theta / 2), \mathbf{R}_{\mathbf{z}}(\theta)\right)<D\left(\tilde{\mathrm{R}}_{\mathbf{z}}(\theta), \mathbf{R}_{\mathbf{z}}(\theta)\right)
$$

doit être satisfaite. Le tilde " ' est utilisé ici pour indiquer les portes logiques avec bruit. 


\subsection{TOLÉRANCE AU BRUIT DES PARAMÈTRES DE CONTRÔLE}

Afin de calculer la distance, on développe $\mathrm{U}(n)$ dans l'expression (2.24) au premier ordre en $\delta B$ et $t / N$. On moyenne ensuite la distance ainsi obtenue en appliquant le théorème de la limite centrale aux variables $X_{i} \equiv \sum_{n=1}^{N} \delta B_{i}(n)$. De plus, on note que le temps nécessaire pour compléter $R_{i}(\phi)$ est $t_{i}=N_{i} \Delta t=\phi / B_{i}$. Pour la porte géométrique, cela conduit à $N_{n} B_{n}=2 N_{x} B_{x}$ puisque les angles de rotation impliqués dans la séquence (2.15) sont $\pi$ et $\pi / 2$ respectivement. De cette façon, en présence de bruit selon $x$ et $z$, on obtient

$$
\begin{aligned}
\left\langle D\left(\tilde{\mathrm{R}}_{\mathbf{z}}^{\mathrm{AA}}(\theta / 2), \mathrm{R}_{\mathbf{z}}(\theta)\right)\right\rangle & \approx \sqrt{\frac{\pi^{3}}{12}\left(\frac{1}{B_{x}^{2}}+\frac{1}{B_{x} B_{n}}\right)} \frac{\delta B_{\max }}{\sqrt{N_{x}}} ; \\
\left\langle D\left(\tilde{\mathrm{R}}_{\mathbf{z}}(\theta), \mathrm{R}_{\mathbf{z}}(\theta)\right)\right\rangle & \approx \sqrt{\frac{\pi}{6}} \frac{\theta \delta B_{\max } / B_{z}}{\sqrt{N_{z}}}
\end{aligned}
$$

où $B_{x}, B_{n}$ et $B_{z}$ sont les amplitudes des champs effectifs utilisées pour réaliser $\mathrm{R}_{\mathrm{x}}(\pi / 2)$, $\mathrm{R}_{\boldsymbol{n}}(\pi)$ et $\mathrm{R}_{\mathbf{z}}(\theta)$ respectivement. Avec une diminution de $N_{i}$, le bruit est constant sur une plus longue portion de l'évolution. Des excursions s'éloignant davantage du parcours original sur la sphère de Bloch sont alors possibles. La distance entre les portes avec et sans bruit augmente donc avec une diminution de $N_{i}$. Plus de détails sur les étapes de calcul nécessaires pour obtenir (2.27b) peuvent être trouvés en annexe B.

La figure 2.3 présente une vérification numérique de ces relations. La faible dépendance de $\left\langle D\left(\tilde{R}_{\mathbf{z}}^{\mathrm{AA}}(\theta / 2), \mathrm{R}_{\mathbf{z}}(\theta)\right)\right\rangle$ sur l'angle $\theta$ en raison de $B_{n}$ est apparente à la figure 2.3 a) [voir annexe $\mathrm{A}$ ]. Pour $\left\langle D\left(\tilde{\mathrm{R}}_{\mathbf{z}}(\theta), \mathrm{R}_{\mathbf{z}}(\theta)\right)\right\rangle$, la dépendance est en $\sqrt{\theta}$ puisque $N_{z} \propto \theta$, figure $2.3 \mathrm{~b}$ ). L'accord entre les résultats analytiques et numériques est très bon, avec des écarts d'environ $3 \%$ dans les deux cas. L'estimation au premier ordre utilisé ici est donc suffisante pour ce niveau de bruit. Les systèmes plus bruyants ne seront fort probablement pas utiles pour le calcul quantique et donc, à toute fin pratique, l'approximation utilisée ici devrait suffire.

En utilisant le résultat (2.27), l'inégalité (2.26) et en supposant que le temps de corrélation du bruit est identique pendant la réalisation des opérations dynamiques et géométriques, on obtient une borne sur l'angle $\theta$ au-delà duquel la porte géométrique devient favorable par rapport à la porte dynamique

$$
\theta_{b}>\pi\left(\frac{B_{z}}{B_{x}}+\frac{B_{z}}{B_{n}}\right)
$$




\subsection{TOLÉRANCE AU BRUIT DES PARAMÈTRES DE CONTRÔLE}
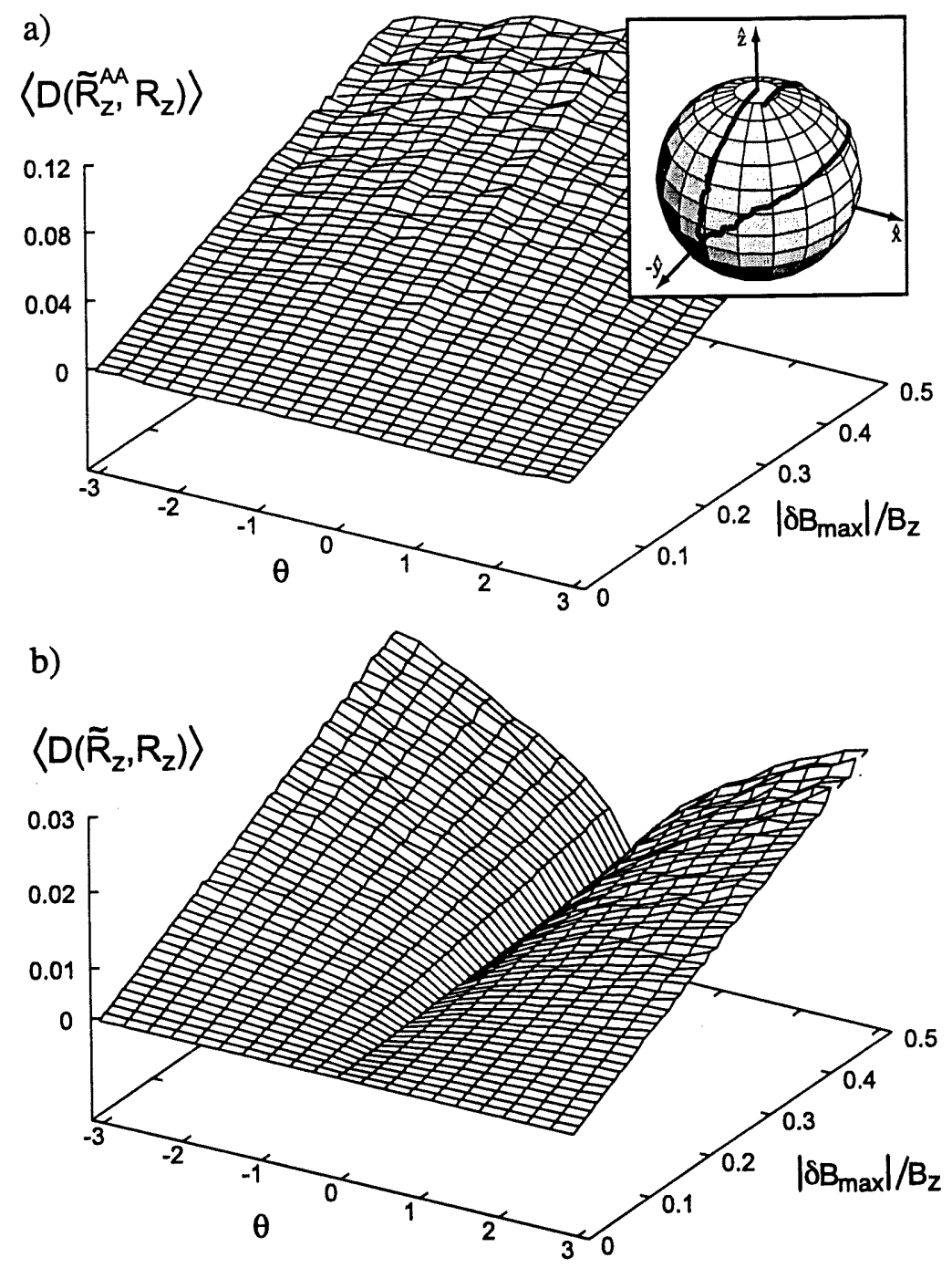

FIG. 2.3: Distance en fonction de $\theta$ et de l'amplitude maximale du bruit. Les résultats sont une moyenne sur 600 réalisations du bruit. Les paramètres de la simulation sont choisis de façon à correspondre à un qubit de charge. Les détails de ces paramètres sont présentés à la figure A.4 de l'annexe A. Le bruit est selon $x$ et $z$ et est exprimé en unité de la valeur maximale du champ effectif dans la direction $z: B_{z}=4 E_{C}$ (voir l'annexe A pour plus de détails). a) Moyenne de la distance entre une porte $\mathrm{AA}$ avec bruit et la porte dynamique idéale correspondante. L'encadré présente un parcours avec bruit. Celui-ci n'est plus fermé et l'évolution n'est pas cyclique. b) Comme en a) mais pour la porte dynamique $\mathrm{R}_{\mathbf{z}}$. Dans les deux cas, le temps de corrélation du bruit est $\Delta t=\hbar /\left(4 E_{C} \gamma\right)$ avec $\gamma=300$. 


\subsection{TOLÉRANCE AU BRUIT DES PARAMÈTRES DE CONTRÔLE}

En prenant $B_{z} / B_{x} \approx B_{z} / B_{n} \approx 1$, on obtient que pour $\theta_{b} \gtrsim 2 \pi$ la porte AA sera moins affectée par le bruit que sa contrepartie dynamique. Pour le qubit de charge, $B_{z}$ et $B_{x}$ sont fixés respectivement par l'énergie de charge $E_{C}$ et l'énergie Josephson $E_{J}$. Afin d'encoder efficacement l'information dans le degré de liberté de charge, l'inégalité $E_{C} \gg E_{J}$ doit être satisfaite [108]. La borne obtenue avec $B_{z} / B_{x} \approx B_{z} / B_{n} \approx 1$ correspond donc à un seuil inférieur sur $\theta_{b}$. Puisque $\theta_{b}>2 \pi$, la porte basée sur la phase géométrique non-adiabatique n'est jamais utile en pratique. En particulier, avec les énergies utilisées à la figure 2.3 , on obtient $\theta_{b} \gtrsim 2.5 \pi$ comme seuil inférieur. De façon plus générale, puisque les états logiques d'un qubit sont les états propres de $\sigma_{z}, B_{z}$ devrait être plus grand que $B_{x}$ afin que la base de calcul soit la 'bonne' base. On peut donc s'attendre à ce que cette limite inférieure s'applique à la plupart des architectures d'ordinateurs quantiques.

Cette conclusion s'applique à la situation où le bruit est selon $x$ et $z$. Des résultats analogues aux relations (2.27) et (2.28) ont toutefois été obtenus dans le cas où le bruit est selon $z$ seulement. Dans ce cas aussi, la porte géométrique est plus sensible au bruit que la porte dynamique.

Notons que l'effet de la décohérence sur la porte AA a aussi été étudié par Nazir et al. [110]. En s'intéressant au cas où l'évolution est non-unitaire, ceux-ci arrivent à la même conclusion concernant la sensibilité de la porte AA au bruit. Puisque leur approche permet de considérer plus de types de bruit que nous ne l'avons fait ici, celleci est plus générale que l'approche décrite dans ce travail. Toutefois, leur analyse est purement numérique. De plus, notre objectif ici était d'inclure que le type de bruit pour lequel la porte AA avait été suggérée tolérante, c'est-à-dire le bruit unitaire autour du parcours.

L'approche employée ici pour étudier l'effet des fluctuations peut aussi être utilisée pour les portes de phase de Berry. Pour ce faire, on considère la séquence utilisée dans l'expérience de RMN de Jones et al. [81] et simplifiée dans [110]. L'hamiltonien du système prend donc la forme

$$
H=\frac{\Delta}{2} \sigma_{z}+\frac{\omega_{1}}{2}\left(\cos \phi \sigma_{x}+\sin \phi \sigma_{y}\right) .
$$

La séquence d'opérations utilisée à la référence [81] commence avec le champ selon l'axe $z\left(\omega_{1}=0\right)$, figure 2.4. Le paramètre $\Delta$ est gardé constant tout au cours de 


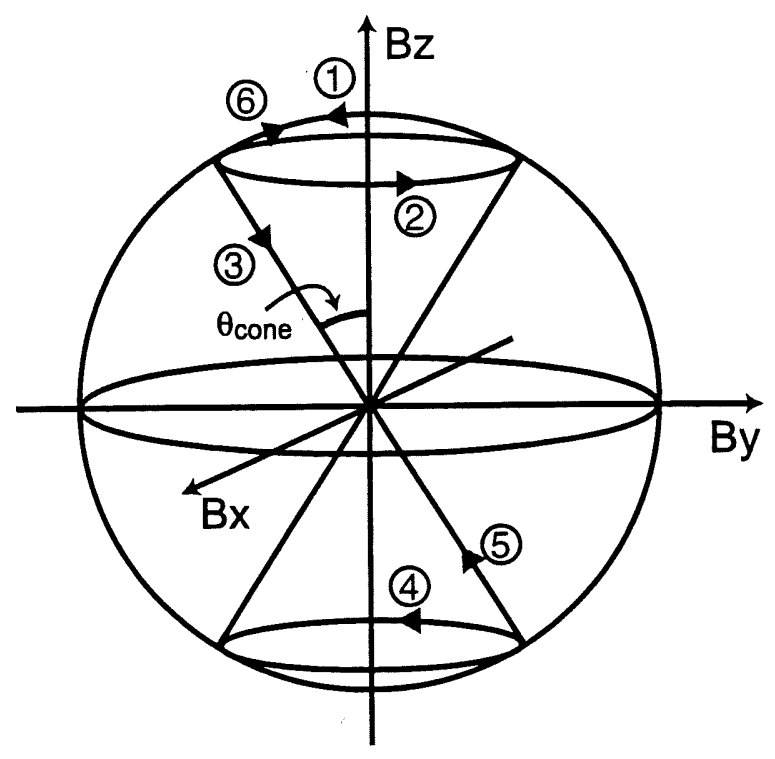

FIG. 2.4: Parcours dans l'espace des paramètres (ne pas confondre ici avec l'espace projectif) utilisé pour réaliser la porte de phase adiabatique avec un hamiltonien tel que l'on trouve en RMN de l'état liquide. Les différentes étapes du parcours sont : (1) Le champ initialement.

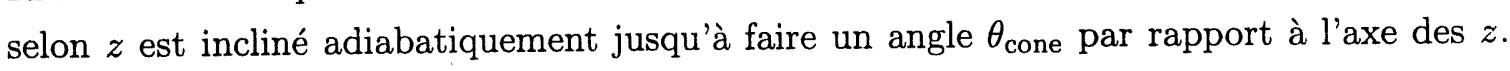
(2) La phase $\phi$ parcours adiabatiquement l'intervalle $0 \rightarrow 2 \pi$. (3) Impulsion $\pi$ rapide. (4) Parcours de $2 \pi$ pour $\phi$. (5) Seconde impulsion $\pi$. (6) Le champ est finalement réaligné avec l'axe $z$.

l'évolution. Le champ est premièrement incliné adiabatiquement dans le plan $x-z$ en augmentant $\omega_{1}$ jusqu'à une valeur maximale $\omega_{1 \text { max }}$. Cette étape se. fait à $\phi=0$ constant. Le champ fait alors un angle $\theta_{\text {cone }}=\arccos \left(\Delta / \sqrt{\Delta^{2}+\omega_{1 \text { max }}^{2}}\right)$ par rapport à l'axe initial. $\grave{A} \omega_{1}$ constant, on fait ensuite parcourir adiabatiquement à $\phi$ l'intervalle $[0,2 \pi]$. Afin d'obtenir une opération purement géométrique, la phase dynamique est annulée en répétant les opérations précédentes dans le sens contraire et entre une paire d'impulsions $\pi$ rapides. Si l'état initial est une superposition des états logiques, la phase relative finale entre ces états sera alors purement géométrique et de grandeur $\gamma=4 \pi\left(1-\cos \theta_{\text {cone }}\right)[81]$.

Afin d'étudier l'effet du bruit sur cette séquence, on utilise la propriété de composition (2.24) et une décomposition de Trotter sur (2.29). On obtient de cette façon, en présence de bruit selon $x, y$ et $z$ et en supposant que les opérations $\mathrm{R}_{\mathrm{y}}(\pi)$ sont 


\subsection{TOLÉRANCE AU BRUIT DES PARAMÈTRES DE CONTRÔLE}

réalisées de façon idéale,

$$
\left\langle D\left(\tilde{\mathrm{R}}_{\mathrm{z}}^{\text {Berry }}(\gamma), \mathrm{R}_{\mathrm{z}}(\gamma)\right)\right\rangle \approx \frac{4}{\sqrt{3 \pi}} \delta B_{\max } \sqrt{\frac{T_{T}^{2}}{N_{T}}+\frac{T_{\phi}^{2}}{N_{\phi}}}
$$

où $T_{T}$ est le temps requis pour incliner le champ dans le plan $x-z$ et $T_{\phi}$ le temps du parcours de $\phi$ dans l'intervalle $[0,2 \pi]$. Comme pour (2.27), plus $N_{T}$ et $N_{\phi}$ sont grands, plus le temps de corrélation du bruit est court. L'accord avec les résultats numériques (non présentés) est excellent. La contrainte d'adiabaticité signifie que $T_{T}$ et $T_{\phi}$ sont grands et donc que, à toute fin pratique, les portes basées sur la phase de Berry sont moins avantageuses que leurs équivalents dynamiques. La conclusion est la même pour tous les types de bruit testés numériquement. Pour les étapes (1) et (4) de la figure 2.4, le bruit selon $x$ seulement et le bruit non corrélé selon $x$ et $z$ ont été testés. Pour les étapes (2) et (4), le bruit identique selon $x$ et $y$ a été utilisé et ce, avec et sans bruit non-corrélé selon $z$. En raison de la contrainte d'adiabaticité, la porte de phase adiabatique est moins performante que la porte AA. C'est la conclusion aussi obtenue à la référence [110] pour des évolutions non unitaires. Notons que la possibilité [82] d'un point d'opération où la phase géométrique conditionnelle est insensible au premier ordre au bruit de $\omega_{1}$ peut être un avantage de la porte de phase adiabatique sur deux qubits. Ce point d'opération semble toutefois limité à des cas très particuliers.

Notons finalement que les résultats principaux de cette section peuvent être compris simplement de façon intuitive. Afin de réaliser les portes logiques basées sur la phase géométrique (adiabatique ou non), il est nécessaire d'appliquer une séquence d'opérations unitaires qui réalise un parcours fermé (dans l'espace des paramètres ou

dans l'espace projectif). En présence de bruit dans les paramètres de contrôle, cette séquence ne correspondra plus à un parcours fermé. Puisque tout ce qui compte pour le calcul quantique est la phase totale, cette phase sera plus affectée par les longues séquences d'opérations que par les séquences plus courtes requises pour réaliser les portes de phases dynamiques. Précisons aussi que si le bruit a une symétrie spéciale qui fait en sorte qu'elle préserve l'aire, alors il est possible que cette symétrie puisse être exploitée plus efficacement par les techniques de correction d'erreurs quantiques, les DFS ou le contrôle bang-bang qu'avec les portes géométriques. 


\subsection{Géométrique vs topologique}

Tel que décrit dans les sections précédentes, on utilise la phase géométrique dans le but de faire des opérations logiques idéales (tolérantes aux imperfections) sur des qubits qui sont eux-même non-idéaux (sensibles aux imperfections). On a toutefois montré à la section précédente que la phase géométrique n'est malheureusement pas un outil bien adapté à ce travail.

Une idée qui semble plus puissante est de concevoir les qubits de façon à ce qu'ils soient eux-même insensibles aux imperfections (de fabrication et de contrôle). Cette idée a été explorée par Kitaev en exploitant le concept de topologie [111]. Celui-ci a montré que la topologie de certains systèmes peut être exploitée de façon à ce que l'espace d'Hilbert se sépare en différents 'secteurs' orthogonaux. De plus, ces secteurs demeurent isolés sous l'effet de perturbations locales $O_{i}$. Ceci implique que les éléments de matrice hors-diagonaux de ces opérateurs s'annulent et que leurs éléments diagonaux ne dépendent pas des états, i.e.

$$
\left\langle m\left|O_{i}\right| n\right\rangle=c_{i} \delta_{m n}
$$

où $|m\rangle$ et $|n\rangle$ appartiennent à des sous-espaces orthogonaux différents et $c_{i}$ est une constante. L'information encodé dans ces différents secteurs n'est alors pas perturbée par les opérations locales. Récemment, Ioffe et al. ont montré comment il est possible, en principe, de réaliser ces concepts à l'aide de réseaux de jonctions Josephson [112, $113]$.

Ce concept de qubits protégés topologiquement semblent particulièrement intéressant puisque la "correction d'erreurs" est alors intégré au qubit même. La protection contre l'environnement se fait passivement plutôt qu'activement comme c'est le cas dans le contexte de la phase géométrique. Il est intéressant de remarquer que la contrainte (2.31) est équivalente à la condition (1.28) imposée sur les codes et les opérateurs du bain dans le contexte des DFS. Ces concepts sont donc intimement reliés. Cette relation a été exploré formellement par Zanardi et Lloyd [114]. Mentionnons aussi que le qubit phase-charge, qui sera décrit en détail à la section $\S 3.4 .3$, utilise, de manière beaucoup plus simple, se concept de tolérance intrinsèque aux imperfections. 


\subsection{Sommaire}

Dans ce chapitre, nous avons considéré la phase d'AA comme outil pour le calcul quantique. Cette phase résout plusieurs des problèmes de la porte de phase adiabatique. Elle peut être réalisée plus rapidement, n'exige pas d'annulation d'une composante dynamique et n'implique que le contrôle de deux champs effectifs de l'hamiltonien à un qubit. Nous avons aussi montré comment la phase AA sur un qubit peut être observée à l'aide d'un second qubit et ce sans phase dynamique supplémentaire. Comme exemple d'application, les détails de la réalisation de ces idées avec un qubit de charge ont été donnés. L'application de ces idées à d'autres architectures d'ordinateurs quantiques est une généralisation simple.

Lorsque l'effet du bruit dans les paramètres de contrôle est pris en considération, il s'avère que les portes logiques basées sur le concept de phase géométrique, adiabatique ou non-adiabatique, sont plus sensibles au bruit que les portes purement dynamiques. Ceci s'oppose à ce qui a été précédemment suggéré. Nous avons vérifié comment le bruit affecte les transformations unitaires globales qui, dans le cas sans imperfection, réalisent les portes logiques purement géométriques. Les résultats analytiques ont été confirmés numériquement pour un éventail de symétries du bruit. Les résultats sont en accord avec les travaux récents de la référence. [110]. Dans ce travail cependant, nous avons concentré notre attention sur le type de bruit pour lequel les portes logiques géométriques avaient été supposées tolérantes.

La phase AA semble donc être de peu d'intérêt pour le calcul quantique. Il est cependant d'intérêt fondamental d'observer cette phase. Une observation directe avec une paire de qubits supraconducteurs de charge semble possible. L'architecture d'ordinateur quantique la plus avancée du point de vue expérimental est, au moment d'écrire ce document, le qubit phase-charge [6] et sera décrit au chapitre suivant. Il serait intéressant d'appliquer les séquences à un et deux qubits pour la phase AA décrite dans ce chapitre à ce type de qubit. De plus, vue le mode de fonctionnement de ce qubit, l'application de la phase géométrique adiabatique peut être réalisée de façon très similaire à ce qui est fait en RMN [81] et qui a été décrit ici. L'observation des phases géométrique adiabatique et non-adiabatique (sur un qubit) semble possible avec les qubits phase-charge déjà disponibles au laboratoire [6]. La réalisation 
de ces expériences constituerait une première observation de phase géométrique dans les systèmes supraconducteurs mésoscopiques. Les résultats de ce chapitre ont été publiés dans Physical Review A [99]. 


\section{Chapitre 3}

\section{Qubits supraconducteurs}

(...) quantum phenomena do not occur in a Hilbert space, they occur in the laboratory.

- Asher Peres

Aux chapitres précédents, nous avons vu qu'un ordinateur quantique peut, en principe, surpasser sa contrepartie classique dans l'exécution de certaines tâches. En parallèle au développement de l'informatique quantique, il a été reconnu au cours des 20 dernières années que les systèmes supraconducteurs, et en particulier les systèmes basés sur les jonctions Josephson, sont de bons candidats pour l'observation de phénomènes quantiques au niveau macroscopique [115]. Il n'est donc pas étonnant que les qubits basés sur les systèmes supraconducteurs suscitent maintenant beaucoup d'attention, tant au niveau théorique qu'expérimental.

Dans ce chapitre, nous présenterons les idées principales reliées aux qubits supraconducteurs. Dans la première section, une approche très utile pour décrire le comportement quantique des circuits électriques est décrite. À titre d'exemple, les circuits correspondant aux qubits de phase et aux qubits de charge sont examinés. Le passage vers un espace d'Hilbert à deux niveaux et l'effet des fluctuations sur ces systèmes sont ensuite étudiés. De cette analyse, il est possible d'obtenir les échelles de temps sur lesquelles on peut espérer que ces systèmes supraconducteurs présentent un comportement quantique. D'autres propositions de qubits supraconducteurs et des expériences récentes sont ensuite discutées. Afin de ne pas alourdir la présentation, 


\subsection{CIRCUITS ÉLECTRIQUES QUANTIQUES}

certaines de nos contributions originales ne seront que brièvement mentionnées dans ce chapitre. Certaines des publications reliées à ces aspects sont toutefois reproduites aux annexes $\mathrm{C}$ et $\mathrm{D}$.

Notons que des articles de revues ont récemment été écrits sur ce sujet $[108,116]$. Plusieurs aspects traités ici sont aussi abordés dans ces articles. Depuis leur publication toutefois, plusieurs propositions et résultats expérimentaux ont été présentés dans la littérature. Plusieurs de ces idées plus récentes se retrouvent dans les compterendus de la conférence SQUID 2001, publiés au volume 363 de Physica C. Des résultats encore plus récents seront décrits à la dernière section de ce chapitre.

\subsection{Circuits électriques quantiques}

Dans cette section, une procédure systématique pour obtenir le lagrangien et l'hamiltonien d'un circuit électrique est décrite. Le passage de l'hamiltonien classique à l'hamiltonien quantique se fait alors simplement par quantification canonique. Évidemment, le comportement quantique d'un circuit électrique est étroitement lié à la question de la dissipation. Celle-ci sera abordée à la section §3.3. L'approche décrite ici est due à $M$. Devoret [117]. On suit ici cette référence de près.

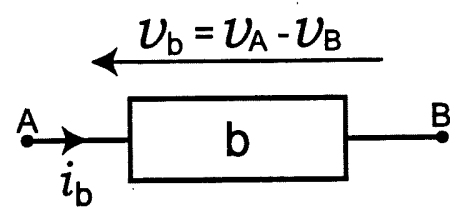

FIG. 3.1: Convention de signes pour les voltages et courants de branche.

Les circuits électriques standards sont des réseaux (graphes) dont les branches sont des éléments discrets à deux terminaux. À chaque élément du réseau, il est possible d'assigner des flux de branche et des charges de branche reliés respectivement au voltage $v_{b}$ à travers la branche $b$ et au courant $i_{b}$ circulant dans $b$ :

$$
\begin{aligned}
& \Phi_{b}(t)=\int_{-\infty}^{t} v_{b}\left(t^{\prime}\right) d t^{\prime}, \\
& Q_{b}(t)=\int_{-\infty}^{t} i_{b}\left(t^{\prime}\right) d t^{\prime} .
\end{aligned}
$$




\subsection{CIRCUITS ÉLECTRIQUES QUANTIQUES}

La convention de signe est illustrée à la figure 3.1. Dans ces relations, on suppose que le circuit est initialement au repos, sans courant ou voltage. Pour les éléments supraconducteurs, le flux de branche $\Phi_{b}$ est relié à la différence de phase à travers l'élément : $\phi=2 \pi \Phi_{b} / \Phi_{0} \bmod 2 \pi$, où $\Phi_{0}=h / 2 e$ est le quantum de flux [118].

Les flux de branche et les charges de branche ne sont pas des variables indépendantes et la description du réseau à l'aide de celles-ci souffre d'une certaine redondance. Les relations entre ces variables dépendent de la topologie du réseau et sont exprimées par la loi de Kirchoff

$$
\begin{aligned}
& \qquad \sum_{\substack{\text { toutes les branches } \\
\text { formant la boucle } l}} \Phi_{b}=\tilde{\Phi}_{l}, \\
& \sum_{\substack{\text { toutes les branches } \\
\text { arrivant au noeud } n}} Q_{b}=\tilde{Q}_{n} .
\end{aligned}
$$

Ces relations expriment le fait que la somme des tensions sur une boucle $l$ et la somme des courants arrivant au noeud $n$ sont nulles lorsque le flux dans la boucle $\tilde{\Phi}_{l}$ et la charge sur le noeud $\tilde{Q}_{n}$ sont constants. Les relations de Kirchoff sont généralement exprimées à l'aide des courants dans la boucle et des voltages au noeud et l'une ou l'autre de ces quantités peut être utilisée pour la description du circuit. Puisqu'elles sont souvent plus appropriées pour décrire les éléments tunnels, nous travaillerons ici avec les variables de noeuds.

Les variables de noeuds dépendent de la description de la topologie du circuit. Suivant toujours la référence [117], la topologie d'un circuit est décrite de la façon suivante. Un noeud de référence est choisi comme masse, les autres noeuds sont alors les noeuds actifs. En partant de la masse, l'arbre générateur $\mathcal{T}$ est construit en connectant chaque noeud actif à la masse de façon telle qu'il n'y ait qu'un chemin de la masse vers chacun des noeuds actifs. Les branches restantes sont alors les branches de fermeture $\mathcal{C}$. Chacune de ces branches définit une boucle irréductible obtenue en joignant les extrémités de la branche de fermeture par le chemin le plus court sur l'arbre générateur. La figure 3.2 présente cette construction dans le cas d'un circuit formé de trois jonctions Josephson. Les branches de l'arbre générateur sont représentées par les lignes pleines et la branche de fermeture par la ligne pointillée. 


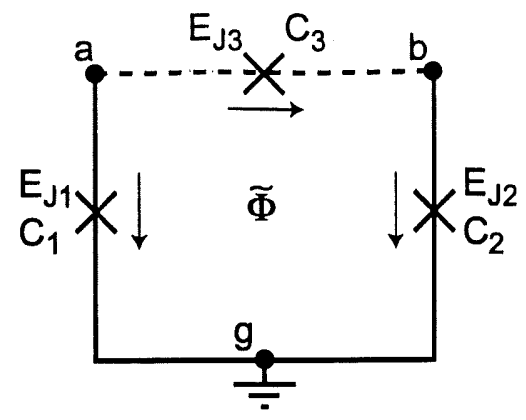

FIG. 3.2: Exemple de circuit électrique : une boucle supraconductrice formée de trois jonctions Josephson. La masse est notée $g$ tandis que $a$ et $b$ sont des noeuds actifs. L'arbre générateur a deux branches : $(a, g)$ et $(b, g)$. Il y a une branche de fermeture $(a, b)$ et par conséquent une seule boucle irréductible (avec un flux $\tilde{\Phi}$ dans la boucle). Nous négligeons ici les éléments dissipatifs (la résistance de shunt des jonctions par exemple) mais considérons explicitement les capacités des jonctions Josephson. De plus, la self-inductance de la boucle est négligée. Puisque celle-ci est distribuée le long de la boucle, elle ne se trouve pas dans la limite des éléments discrets ('lump element') et l'inclure dans la description du circuit peut parfois introduire des complications additionnelles. Cette question sera abordée plus loin.

Évidemment, le choix de la masse et donc de la topologie du circuit n'est pas unique. Les prédictions physiques obtenues d'une description alternative sont toutefois les mêmes (les hamiltoniens obtenus sont reliés par transformation canonique [119]).

On introduit maintenant le flux $\phi_{n}$ du noeud $n$. Cette quantité est définie par intégration temporelle du voltage le long du chemin reliant le noeud à la masse sur l'arbre générateur

$$
\phi_{n}=\sum_{\mathrm{b}} S_{n b} \Phi_{b}
$$

Dans cette expression, $S_{n b}=1,-1$ ou 0 selon que le chemin joignant la masse à $n$. suit la branche $b$ dans la bonne orientation, avec l'orientation opposée ou ne suit pas b. Évidemment, à partir de cette relation, il est possible d'écrire le flux de la branche $b$ à l'aide du flux des noeuds $n$ et $n^{\prime}$ à ses extrémités

$$
\begin{aligned}
& \Phi_{b \in \mathcal{T}}=\phi_{n}-\phi_{n^{\prime}} \\
& \Phi_{b \in \mathcal{C}}=\phi_{n}-\phi_{n^{\prime}} \pm \tilde{\Phi}_{l} .
\end{aligned}
$$

Le signe associé au flux extérieur $\tilde{\Phi}_{l}$ dépend de l'orientation du champ magnétique 


\subsection{CIRCUITS ÉLECTRIQUES QUANTIQUES}

qui le génère. De la loi de Faraday, ce signe est simplement l'inverse de celui donné par la règle de la main droite.

À l'aide de ces définitions, il est simple d'obtenir le lagrangien d'un circuit. Pour une branche $b$ ayant à ses extrémités les noeuds $n$ et $n^{\prime}$, on ajoute au lagrangien un des termes suivants selon le type de $b$ :

Capacité

Inductance

Jonction Josephson

$$
\begin{array}{ccc}
\frac{1}{2} C_{b} V_{b}^{2} & \rightarrow & \frac{1}{2} C_{b}\left(\dot{\phi}_{n}-\dot{\phi}_{n^{\prime}}\right)^{2} \\
-\frac{\Phi_{b}^{2}}{2 L_{b}} & \rightarrow & -\frac{\left(\phi_{n}-\phi_{n^{\prime}}+\bar{\Phi}_{l(b)}\right)^{2}}{2 L_{b}}
\end{array}
$$$$
E_{J} \cos \varphi_{b} \quad \rightarrow \quad E_{J} \cos \frac{2 \pi}{\Phi_{0}}\left(\phi_{n}-\phi_{n^{\prime}}+\tilde{\Phi}_{l(b)}\right) \text {. }
$$

Pour les jonctions Josephson, on doit tenir compte de l'énergie de charge et de l'énergie Josephson. Le lagrangien du circuit de la figure 3.2 est alors

$$
\begin{aligned}
L= & \frac{1}{2} C_{1} \dot{\phi}_{a}^{2}+\frac{1}{2} C_{2} \dot{\phi}_{b}^{2}+\frac{1}{2} C_{3}\left(\dot{\phi}_{a}-\dot{\phi}_{b}\right)^{2} \\
& +E_{J 1} \cos \frac{2 \pi}{\Phi_{0}} \phi_{a}+E_{J 2} \cos \frac{2 \pi}{\Phi_{0}} \phi_{b}+E_{J 3} \cos \frac{2 \pi}{\Phi_{0}}\left(\phi_{a}-\phi_{b}+\tilde{\Phi}\right),
\end{aligned}
$$

où le flux du noeud de référence est choisi comme $\phi_{g}=0$. Les flux de branche sont donc simplement $\Phi_{(a, g)}=\phi_{a}$ et $\Phi_{(b, g)}=\phi_{b}$.

Le circuit qui nous intéresse ici est formé de trois îles supraconductrices. Il est donc décrit par trois phases supraconductrices. En choisissant un noeud de référence, une variable a été éliminée et il ne reste plus que $\phi_{a}$ et $\phi_{b}$ dans le lagrangien. Cette élimination concorde avec la contrainte imposée par la quantification du flux [118]. En effet, la quantification du flux impose aux différences de phase d'obéir à la relation $\varphi_{1}+\varphi_{2}+\varphi_{3}=\left(2 \pi / \Phi_{0}\right) \Phi=\left(2 \pi / \Phi_{0}\right) L I+\left(2 \pi / \Phi_{0}\right) \tilde{\Phi}$, où $\varphi_{i}$ est la différence de phase à travers la jonction $i, L$ la self de la boucle et $I$ le courant circulant dans la boucle. De plus, $\Phi$ est le flux total dans la boucle, $L I$ le flux induit par le courant $I$ et $\tilde{\Phi}$ le flux appliqué de l'extérieur. Puisque l'on néglige ici la self, alors $\Phi \approx \tilde{\Phi}$ et $\varphi_{3} \approx\left(2 \pi / \Phi_{0}\right) \tilde{\Phi}-\varphi_{2}-\varphi_{3}$. Il ne reste donc, comme il se doit, que deux variables (les signes appropriés peuvent être inclus dans les différences de phase $\varphi_{i}$ ).

Du lagrangien, on passe facilement à l'hamiltonien à l'aide de la transformation de Legendre

$$
H=\sum_{n} p_{n} \dot{\phi}_{n}-L
$$




\subsection{CIRCUITS ÉLECTRIQUES QUANTIQUES}

où $p_{n}$ est le moment conjugué à $\phi_{n}$

$$
p_{n} \equiv \frac{\partial L}{\partial \dot{\phi}_{n}} .
$$

Pour les circuits électriques, ces moments conjugués sont les sommes des charges sur les capacités connectées au noeud correspondant. Afin que ces moments soient bien définis, un noeud ne doit donc pas être relié uniquement à des inductances. En pratique, ce ne sera jamais le cas.

À l'aide de cette transformation et du lagrangien (3.7), on obtient l'hamiltonien correspondant au circuit de la figure 3.2

$$
\begin{aligned}
H= & \frac{1}{C_{1} C_{2}+C_{1} C_{3}+C_{2} C_{3}}\left(\frac{\left(C_{2}+C_{3}\right) p_{a}^{2}}{2}+\frac{\left(C_{1}+C_{3}\right) p_{b}^{2}}{2}+C_{3} p_{a} p_{b}\right) \\
& -E_{J 1} \cos \frac{2 \pi}{\Phi_{0}} \phi_{a}-E_{J 2} \cos \frac{2 \pi}{\Phi_{0}} \phi_{b}-E_{J 3} \cos \frac{2 \pi}{\Phi_{0}}\left(\phi_{a}-\phi_{b}+\tilde{\Phi}\right) .
\end{aligned}
$$

Les capacités effectives associées aux termes $p_{a(b)}^{2}$ peuvent aussi s'obtenir facilement en calculant la capacité entre le noeud $a(b)$ et la masse $g$ à l'aide des règles standards de combinaison des capacités en séries et en parallèles. Les capacités croisées, ici le coefficient du terme bilinéaire $p_{a} p_{b}$, sont souvent plus difficiles à obtenir par une analyse de circuit standard. Un des avantages de cette approche est justement d'obtenir ces capacités plus facilement.

Il est intéressant de remarquer que, à toute fin pratique, la partie cinétique du lagrangien sera toujours quadratique en 'vitesse' généralisée $\dot{\phi}_{n}$. Le passage du lagrangien à l'hamiltonien fera donc toujours intervenir, peu importe le circuit, les mêmes étapes algébriques (voir, par exemple, la section $\S 8.1$ de la référence [120]). Cette procédure peut donc être facilement automatisée en utilisant un logiciel de manipulation symbolique comme MATHEMATICA ${ }^{1}$.

L'hamiltonien (3.10) est l'hamiltonien classique du circuit électrique de la figure 3.2. Les variables $\phi_{n}$ et $p_{n}$ sont conjuguées canoniques

$$
\left\{\phi_{n}, p_{m}\right\}=\delta_{n, m}
$$

\footnotetext{
${ }^{1}$ Un tel programme a été réalisé par Samuel Marchand, lors d'un stage d'étude au cours de l'été 2002.
} 


\subsection{CIRCUITS ÉLECTRIQUES QUANTIQUES}

(ici, $\{\cdot, \cdot\}$ est le crochet de Poisson) et elles obéissent aux équations du mouvement de Hamilton

$$
\dot{\phi}_{n}=\frac{\partial H}{\partial p_{n}}, \quad \dot{p}_{n}=\frac{\partial H}{\partial \phi_{n}} .
$$

La quantification se fait facilement. Les variables classiques sont simplement remplacées par des opérateurs satisfaisant les relations de commutation

$$
\left[\hat{\phi}_{n}, \hat{p}_{m}\right]=i \hbar \delta_{n, m}
$$

Par la suite, les ' ' ' seront omis lorsque aucune confusion n'est possible.

Le circuit étudié dans cette section n'a pas été utilisé comme exemple sans raisons. Il s'agit en fait d'un qubit de phase supraconducteur [72,121]. Avant d'expliquer comment celui-ci peut être utilisé comme qubit, examinons un second exemple.

\subsubsection{Exemple additionnel : la boîte de Cooper}

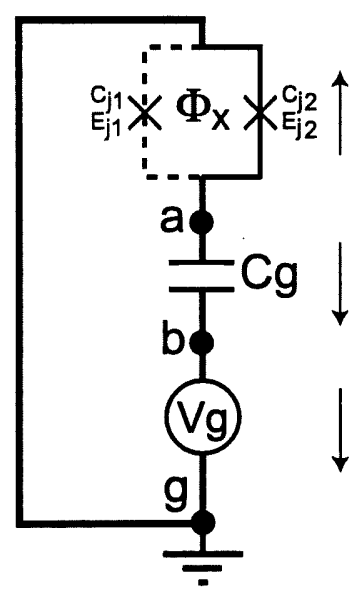

FIG. 3.3: Le noeud $a$ est isolé électriquement du reste du circuit et forme une boîte de Cooper. La ligne pointillée indique la branche de fermeture. Les flux de branche sont définis avec la convention des signes indiquée par les flèches.

Le circuit de la figure 3.3 est connu sous le nom de boîte de Cooper. Si l'énergie du gap supraconducteur est plus grande que toutes les autres énergies du problème (celles-ci sont introduites plus loin), l'effet tunnel de quasiparticules est prohibé. Il s'agit de l'effet de parité $[122,123]$. S'il y a initialement un nombre pair d'électrons sur 


\subsection{CIRCUITS ÉLECTRIQUES QUANTIQUES}

l'île de la boîte (noeud 'a' de la figure 3.3), alors à suffisamment basse température, il n'y a pas de quasiparticules sur celle-ci. L'île contient alors un nombre discret $n$ de paires de Cooper (par rapport à une référence neutre). Avant de donner davantage de détails sur les particularités de ce circuit, déterminons d'abord son hamiltonien.

À partir de la figure 3.3, il est simple d'écrire le lagrangien de ce circuit. Puisqu'on y reviendra à plusieurs reprises au cours des chapitres suivants, on présente néanmoins les étapes principales du calcul. Premièrement, on définit les flux de branche $\Phi_{n m}$ entre les noeuds $n$ et $m$ à l'aide des relations (3.1) et (3.5):

$$
\begin{aligned}
\Phi_{b g} & =\phi_{b}-\phi_{g}=\int V_{g} d t \Rightarrow \phi_{b}=\int V_{g} d t \\
\Phi_{a b} & =\phi_{a}-\phi_{b}=\phi_{a}-\int V_{g} d t \\
\Phi_{a g \in \mathcal{T}} & =\phi_{a}-\phi_{g}=\phi_{a} \\
\Phi_{a g \in \mathcal{C}} & =\phi_{a}-\phi_{g}+\Phi_{x}=\phi_{a}+\Phi_{x},
\end{aligned}
$$

où l'on a pris $\phi_{g}=0$. Il ne reste donc plus que $\phi_{a}$ comme variable dynamique.

À l'aide de ces flux de branche et de la prescription (3.6), on obtient le lagrangien suivant

$$
L=\frac{1}{2} C_{g}\left(\dot{\phi}_{a}-V_{g}\right)^{2}+\frac{1}{2} C_{J 1} \dot{\phi}_{a}^{2}+\frac{1}{2} C_{J 2} \dot{\phi}_{a}^{2}+E_{J 1} \cos \frac{2 \pi}{\Phi_{0}}\left(\phi_{a}+\Phi_{x}\right)+E_{J 2} \cos \frac{2 \pi}{\Phi_{0}} \phi_{a} .
$$

En prenant $E_{J 1}=E_{J 2} \equiv E_{J}$, on peut réécrire l'énergie 'potentielle' sous la forme

$$
\begin{aligned}
\cos \frac{2 \pi}{\Phi_{0}} \phi_{a}+\cos \frac{2 \pi}{\Phi_{0}}\left(\phi_{a}+\Phi_{x}\right) & =\cos \frac{2 \pi}{\Phi_{0}}\left(\phi_{a}-\frac{\Phi_{x}}{2}+\frac{\Phi_{x}}{2}\right)+\cos \frac{2 \pi}{\Phi_{0}}\left(\phi_{a}+\frac{\Phi_{x}}{2}+\frac{\Phi_{x}}{2}\right) \\
& =2 \cos \frac{\pi \Phi_{x}}{\Phi_{0}} \cos \frac{2 \pi}{\Phi_{0}}\left(\phi_{a}+\frac{\Phi_{x}}{2}\right) \\
& \rightarrow 2 \cos \frac{\pi \Phi_{x}}{\Phi_{0}} \cos \frac{2 \pi}{\Phi_{0}} \phi_{a} .
\end{aligned}
$$

La translation de $\phi_{a} \rightarrow \phi_{a}-\Phi_{x} / 2$ effectuée à la dernière étape n'affecte pas la dynamique du circuit. De cette dernière relation, il apparaît que le SQUID-dc agit comme une jonction Josephson ayant une énergie Josephson $2 E_{J} \cos \pi \Phi_{x} / \Phi_{0}$ ajustable à l'aide du flux extérieur. Comme dans l'exemple précédent, cette relation peut être 


\subsection{RÉDUCTION VERS UN SYSTÈME À DEUX NIVEAUX}

obtenue à partir de la condition de quantification du flux dans le cas d'une boucle d'inductance négligeable.

Du lagrangien, on obtient la charge de noeud $p_{a}=C_{\Sigma} \dot{\phi}_{a}-C_{g} V_{g}$ et l'hamiltonien

$$
H=\frac{\left(p_{a}+C_{g} V_{g}\right)^{2}}{2 C_{\Sigma}}-2 E_{J} \cos \frac{\pi \Phi_{x}}{\Phi_{0}} \cos \frac{2 \pi}{\Phi_{0}} \phi_{a},
$$

où $C_{\Sigma} \equiv C_{g}+C_{J 1}+C_{J 2}$ est la capacité totale de l'île. En définissant $n \equiv p_{a} / 2 e$ et $n_{g} \equiv-C_{g} V_{g} / 2|e|$, où $n$ est le nombre de paires de Cooper en excès sur l'île et $n_{g}$ est la charge de grille adimensionnelle, l'hamiltonien se réécrit comme

$$
H=4 E_{C}\left(n-n_{g}\right)^{2}-2 E_{J} \cos \frac{\pi \Phi_{x}}{\Phi_{0}} \cos \frac{2 \pi}{\Phi_{0}} \phi .
$$

Ici, $E_{C} \equiv e^{2} / 2 C_{\Sigma}$ est l'énergie de charge de l'île et $\phi \equiv \phi_{a}$. Après avoir effectué le remplacement standard entre les variables classiques et les opérateurs, on obtient l'hamiltonien quantique de la boîte de Cooper.

\subsection{Réduction vers un système à deux niveaux}

L'approche systématique décrite à la section précédente peut être utilisée pour obtenir facilement l'hamiltonien de circuits électriques. Toutefois, afin que ces circuits soient utiles pour le calcul quantique, il est nécessaire d'isoler un système à deux niveaux agissant comme qubit. Dans cette section, on présente cette réduction premièrement pour la boîte de Cooper puis pour la boucle supraconductrice à trois jonctions.

\subsubsection{Qubit de Charge}

Pour l'hamiltonien (3.18) de la boîte de Cooper, deux échelles d'énergie sont en compétition : l'énergie de charge $E_{C}$ et l'énergie Josephson $E_{J}$. D'autres énergies importantes sont le gap supraconducteur $\Delta$ et la température $k_{B} T$. Dans la représentation de phase, on fait le remplacement $n \rightarrow-i \partial / \partial \phi[118]$ et (3.18) est réécrit sous la forme

$$
H=4 E_{C} \frac{\partial^{2}}{\partial \phi^{2}}-E_{J}\left(\Phi_{x}\right) \cos \frac{2 \pi}{\Phi_{0}} \phi
$$




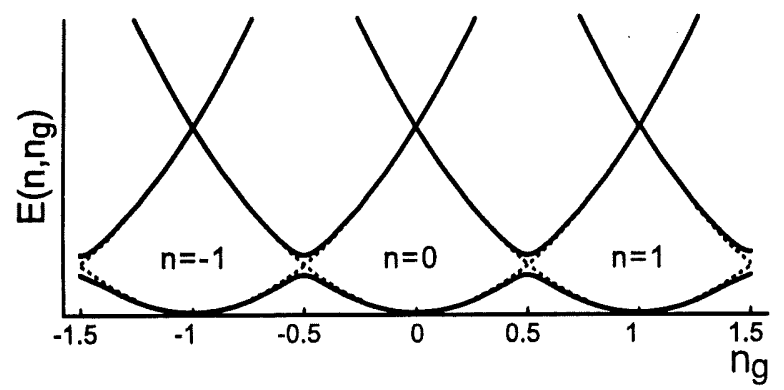

(a)

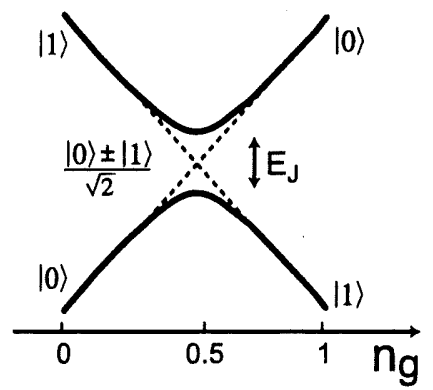

(b)

FIG. 3.4: a) Énergie de la boîte de Cooper (unitées arbitraires) : lignes pleines $E_{C} / E_{J}=10$, lignes en traits brisés $E_{C} / E_{J}=100$. Lorsque la charge de grille adimensionnelle s'approche des valeurs demi-entières, l'interaction Josephson mélange de façon cohérente les états de charges adjacentes de la boîte et lève la dégénérescence de $E_{J}$. b) En se limitant aux voltages de grille tels que $n_{g} \in[0,1]$, la boîte est limitée à deux états de charge. Ceux-ci peuvent jouer le rôle d'états logiques pour un qubit.

avec $E_{J}\left(\Phi_{x}\right) \equiv 2 E_{J} \cos \left(\pi \Phi_{x} / \Phi_{0}\right)$ et où l'on ne tient pas compte de la charge de grille adimensionnelle pour l'instant. Dans cette représentation, il apparaît clairement que l'énergie de charge joue le rôle d'énergie cinétique. Celle-ci tente de délocaliser la 'particule'. À l'opposé, le terme proportionnel à l'énergie Josephson joue le rôle d'une énergie potentielle localisant la particule dans un des puits dù cosinus. Ainsi, les systèmes ayant une grande capacité $C_{\Sigma}$, de sorte que l'énergie Josephson domine, ont une phase bien définie. D'autre part, pour les systèmes ayant une faible capacité, et par conséquent $E_{C}>E_{J}$, la particule n'a pas une phase bien définie. La charge, variable conjuguée à la phase, est alors le bon nombre quantique. Il s'agit d'une manifestation de la relation d'incertitude charge-phase : $\Delta n \Delta \phi \gtrsim 1$; voir la Réf. [118] et la section $\S 1.4 .3$ de la Réf. [123] à ce sujet.

Pour les qubits supraconducteurs de charge, on choisit les paramètres du système de telle sorte que $\Delta \gg E_{C}>E_{J}>k_{B} T$. Dans ce cas, il est utile de développer l'hamiltonien dans la base de charge

$$
|n\rangle=\int_{0}^{2 \pi} \frac{d \phi}{2 \pi} e^{+i n \phi}|\phi\rangle .
$$




\subsection{RÉDUCTION VERS UN SYSTÈME À DEUX NIVEAUX}

Avec

$$
|\phi\rangle=\sum_{n=-\infty}^{\infty} e^{-i n \phi}|n\rangle
$$

et $e^{i \hat{\phi}}|\phi\rangle=e^{i \phi}|\phi\rangle$, on vérifie facilement que $e^{i \hat{\phi}}|n\rangle=|n+1\rangle$. En écrivant le terme Josephson sous la forme $-E_{J} \cos \hat{\phi}=-E_{J}\left(e^{i \hat{\phi}}+e^{-i \hat{\phi}}\right) / 2$, on obtient finalement pour la boîte de Cooper $[108,118]$ :

$$
H=\sum_{n}\left[4 E_{C}\left(n-n_{g}\right)^{2}|n\rangle\langle n|-\frac{1}{2} E_{J}\left(\Phi_{x}\right)(|n\rangle\langle n+1|+| n+1\rangle\langle n|)\right] .
$$

La figure 3.4 a) présente l'énergie de charge (paraboles en traits brisés) en fonction de la charge de grille et ce, pour différentes valeurs du nombre de charge $n$ sur l'île. Aux voltages de grille tels que $n_{g}=k / 2$ où $k=0, \pm 1, \pm 2 \ldots$, l'énergie de charges adjacentes $n$ et $n \pm 1$ est identique. L'effet tunnel cohérent de paires de Cooper connecte ces états adjacents et lève la dégénérescence de $E_{J}$. Loin des points de dégénérescence, l'énergie de charge domine.

Si l'on restreint notre attention à un petit intervalle de voltage autour de $n_{g}=1 / 2$ alors seulement deux états de charge sont importants : $n=0$ et $n=1$. Pour ces voltages de grille, les autres états de charge ont des énergies beaucoup plus élevées et, à toute fin pratique, peuvent être éliminés de la discussion. Par conséquent, en travaillant avec une île de petite capacité totale (grande énergie de charge) et en limitant la tension à l'intervalle $n_{g} \in[0,1]$, il est possible de restreindre la description de la boîte de Cooper à un système à deux niveaux. Cette situation est illustré à la figure $3.4 \mathrm{~b}$ ). On complète le passage vers le langage des pseudo-spins en effectuant les remplacements suivants dans l'hamiltonien :

$$
n \rightarrow \frac{1-\sigma_{z}}{2}, \quad \cos \frac{2 \pi}{\Phi_{0}} \phi \rightarrow \frac{\sigma_{x}}{2}, \quad \sin \frac{2 \pi}{\Phi_{0}} \phi \rightarrow \frac{\sigma_{y}}{2}
$$

pour finalement obtenir de (3.22)

$$
H=-\frac{1}{2} B_{z} \sigma_{z}-\frac{1}{2} B_{x} \sigma_{x}
$$

Les champs effectifs sont ici

$$
\begin{array}{r}
B_{z} \equiv 4 E_{C}\left(1-2 n_{g}\right), \\
B_{x} \equiv 2 E_{J} \cos \left(\pi \Phi_{x} / \Phi_{0}\right) .
\end{array}
$$


La manipulation de l'hamiltonien du pseudo-spin 1/2 est donc possible en ajustant le voltage de grille et le flux externe [108]. Les états de charge $n=0,1$ peuvent ainsi jouer le rôle d'états logiques pour un qubit. On réalisera, par exemple, une rotation du qubit selon $x$ et d'un angle $\theta=2 E_{J} \tau$ en ajustant la charge de grille à $n_{g}=1 / 2$ et le flux à $\Phi_{x}=0$ pendant un temps $\tau$.

\subsubsection{Qubit de phase}

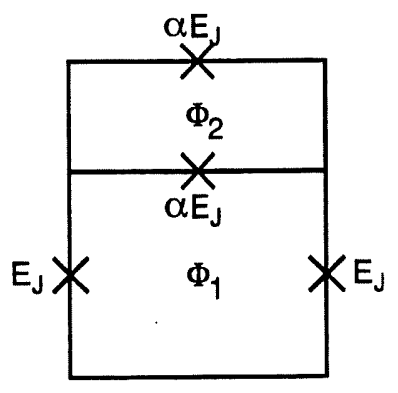

FIG. 3.5: Design modifié pour le qubit de phase à trois jonctions. Par rapport aux jonctions sur les branches verticales, les jonctions sur les branches horizontales ont une énergie Josephson plus petite par un facteur $\alpha$. Cette dernière paire de jonctions agit comme une seule jonction ayant une énergie ajustable par le flux $\Phi_{2}$. Les flux externes $\Phi_{1}$ et $\Phi_{2}$ sont ajustables indépendamment.

Pour le circuit de la figure 3.2, on choisit de travailler avec des jonctions ayant une plus grande surface de sorte que $E_{J} \gg E_{C}$. Dans ce cas, l'hamiltonien (3.10) décrit une particule évoluant dans l'espace bidimensionnel $\left\{\phi_{a}, \phi_{b}\right\}$. Le rôle de la partie cinétique de l'hamiltonien est de fournir une 'masse' à la particule tandis que la partie Josephson décrit le potentiel dans lequel la particule évolue.

Afin que ce circuit soit utile pour le calcul, il est pratique de remplacer une des jonctions du circuit de la figure 3.2 par une paire de jonctions. Ce circuit est présenté à la figure 3.5 [72]. Tel qu'expliqué à la section précédente, cette paire agit comme une jonction unique ayant une énergie Josephson ajustable par le flux externe $\Phi_{2}$. Cette dépendance donne un degré de liberté additionnel utile dans la manipulation du qubit. L'énergie potentielle pour ce design modifié est présentée à la figure $3.6 \mathrm{a}$ ). Le potentiel est une fonction périodique selon les directions $\tilde{\phi}_{a}=2 \pi \phi_{a} / \Phi_{0}$ et $\tilde{\phi}_{b}=2 \pi \phi_{b} / \Phi_{0}$. 


\subsection{RÉDUCTION VERS UN SYSTÈME À DEUX NIVEAUX}

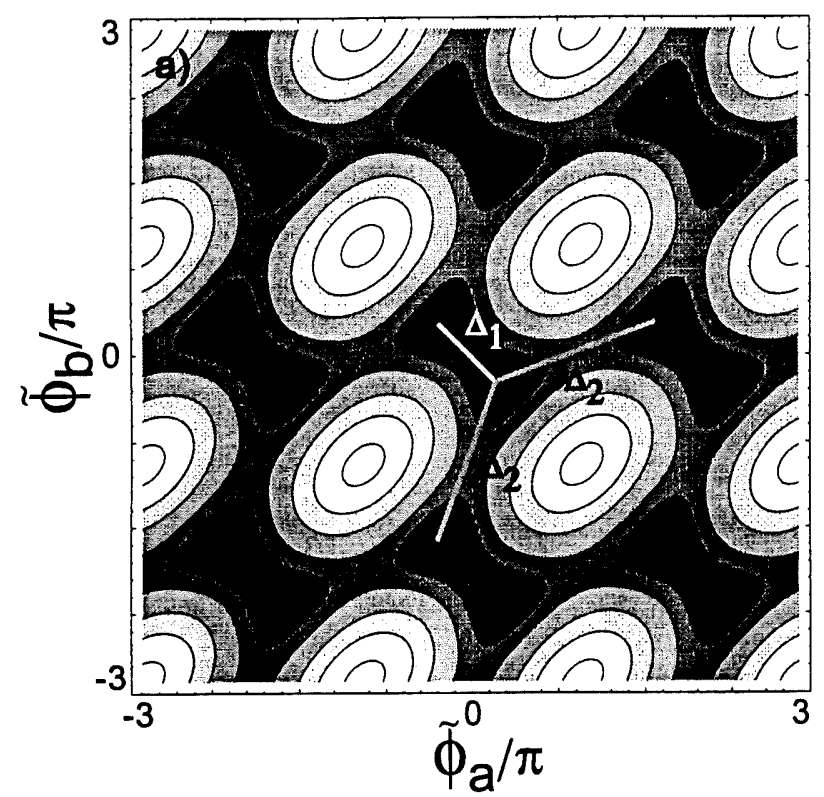

b)

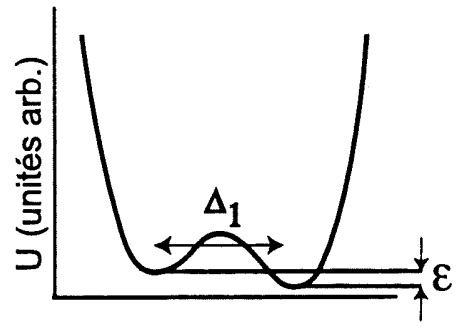

c)

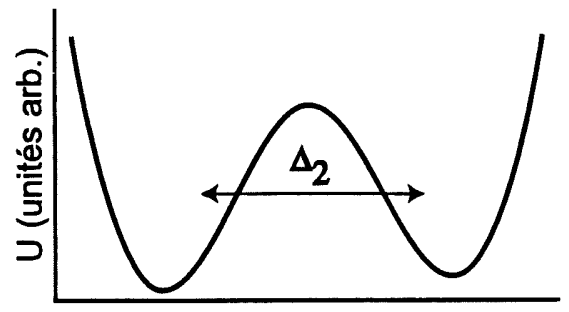

FIG. 3.6: a) Courbes de niveaux de l'énergie potentielle en fonction de $\tilde{\phi}_{a}=2 \pi \phi_{a} / \Phi_{0}$ et $\tilde{\phi}_{b}=2 \pi \phi_{b} / \Phi_{0}$ pour le design modifié de la figure 3.5. Ici, $\alpha=0.75$ et $\pi \Phi_{1} / \Phi_{0}=\pi \Phi_{2} / \Phi_{0}=$ 0.33 . b) Énergie potentielle le long de l'axe principal d'une cellule unité. La barrière de potentielle $U_{b}$ est faible et l'amplitude tunnel $\Delta_{1}$ est par conséquent grande. Les niveaux dans chacun des puits sont alignés $(\varepsilon=0)$ lorsque le flux extérieur est $\Phi_{1}=\Phi_{0} / 2$. c) La barrière de potentiel entre deux cellules unités est grande. L'amplitude tunnel $\Delta_{2}$ dans cette direction est donc exponentiellement supprimée et les cellules unités sont découplées. Figure adaptée de la référence [72].

Chacune des cellules unités est formée de deux puits séparés par une faible barrière de potentiel, figure $3.6 \mathrm{~b}$ ). Si la particule n'est pas trop massive et la barrière est suffisamment petite, la particule peut passer d'un puits à l'autre par effet tunnel cohérent. L'amplitude tunnel peut être évaluée en utilisant les approches WKB ou instanton [124]. Dans ce calcul, il est important de prendre en considération le fait que la masse effective de la particule dépend de la direction dans laquelle elle se déplace dans l'espace $\left\{\tilde{\phi}_{a}, \tilde{\phi}_{b}\right\}$ [121]. Les barrières de potentiel entre les cellules unités sont grandes et l'effet tunnel entre celles-ci est peu probable, figure $3.6 \mathrm{c}$ ). Les cellules unités sont donc à toute fin pratique découplées.

De ce fait, il est possible de restreindre notre attention à un seul double puits de 
potentiel, présenté à la figure 3.6 b). Si les inégalités [124]

$$
U_{b} \gg \hbar \omega_{p} \gg \Delta_{1},|\varepsilon|, k_{B} T,
$$

sont respectées, alors le système ne pourra occuper que le fondamental de chacun des puits. Dans cette expression, $U_{b}$ est la barrière de potentiel, $\hbar \omega_{p}$ la séparation en énergie entre le fondamental et le premier état excité dans chacun des puits (où $\omega_{p} \sim \sqrt{8 E_{C} E_{J}} / \hbar$ est la fréquence des petites oscillations autour du minimum d'un puits), $\Delta_{1}$ l'amplitude tunnel et $\varepsilon$ l'asymétrie. L'espace d'Hilbert a donc, à toute fin pratique, seulement deux dimensions et une bonne base est formée par les états $\{|G\rangle,|D\rangle\}$ localisés dans le puits de gauche et de droite respectivement. Dans cette base, l'hamiltonien prend la forme usuelle

$$
H=-\frac{1}{2} \varepsilon \sigma_{z}-\frac{1}{2} \Delta_{1} \sigma_{x}
$$

L'asymétrie $\varepsilon=2 I_{s}\left(\Phi_{1}-\Phi_{0} / 2\right)$ peut être ajustée à l'aide du flux $\Phi_{1}$ [125]. Dans cette relation, $I_{s}$ est le supercourant dans la boucle du qubit. La hauteur de la barrière de potentiel $U_{b}$, et ainsi l'amplitude tunnel, peut être ajustée à l'aide de $\Phi_{2}$. Ceci complète le passage vers le langage des pseudo-spins. Les états $\{|G\rangle,|D\rangle\}$ qui seront notés $\{|0\rangle,|1\rangle\}$ peuvent servir d'états logiques pour un qubit. Ceux-ci correspondent à un supercourant circulant dans le sens horaire ou anti-horaire dans la boucle à trois jonctions [72].

Le qubit de phase, pour lequel $E_{J} \gg E_{C}$, ainsi que le qubit de charge, avec $E_{C}>E_{J}$, appartiennent à deux différentes classes de qubits supraconducteurs. Dans chacune de ces classes, il existe différents designs ayant chacun leurs avantages et inconvénients. Ces designs alternatifs seront présentés en plus de détails à la section §3.4. Mentionnons qu'un design pour lequel $E_{J} \sim E_{C}$ a récemment été proposé [126] et testé expérimentalement [6]. Celui-ci semble présenter plusieurs avantages qui seront aussi abordés dans une section suivante.

\subsection{Relaxation et déphasage}

Évidemment, pour les systèmes de l'état solide, la réduction vers un espace d'Hilbert à deux niveaux ne peut pas conduire à une description complète. En effet, ces 


\subsection{RELAXATION ET DÉPHASAGE}

systèmes ont plusieurs degrés de liberté. En particulier, les circuits électriques décrits à la section précédente seront contrôlés par des sources extérieures de tensions et de courants. Ces sources sont des appareils classiques à la température ambiante et reliés au circuit quantique par diverses étapes de filtration à basse température. Cette interaction mènera à l'enchevêtrement du qubit avec les degrés de liberté de l'environnement. Puisque qu'il n'est pas possible, en pratique, de contrôler tous ces degrés de liberté, cet enchevêtrement mène à la décohérence. Plus précisément, cela conduit à la relaxation et au déphasage du qubit sur des échelles de temps différentes.

Les taux correspondant à ces processus peuvent être définis à l'aide de la matrice densité réduite du qubit

$$
\rho_{\text {qubit }}=\operatorname{Tr}_{\text {env }}\left\{\rho_{\text {total }}\right\}=\left(\begin{array}{cc}
\rho_{00} & \rho_{01} \\
\rho_{11} & \rho_{11}
\end{array}\right) .
$$

Suite à l'interaction qubit-bain, la matrice densité totale $\rho_{\text {total }}$ ne peut généralement pas s'écrire comme un produit tensoriel $\rho_{\text {qubit }} \otimes \rho_{\text {env }}$. Prendre la trace sur les degrés de liberté du bain conduit alors à une évolution non-unitaire du point de vue du qubit. Le taux de relaxation $\Gamma_{1}$ (aussi noté $\Gamma_{r}$ ) résultant de cette interaction est le taux auquel les éléments diagonaux de $\rho$ atteignent leur valeur d'équilibre. Le taux de déphasage $\Gamma_{2}\left(\Gamma_{\phi}\right)$ est celui auquel les éléments hors diagonaux s'annulent. Les échelles de temps correspondantes ont la même interprétation que les temps $T_{1}$ et $T_{2}$ bien connus en RMN [28].

Pour les circuits électriques décrits aux sections précédentes, l'effet d'une source de voltage (courant) non-idéale et des fils connectant la source au circuit peut être modélisé en ajoutant une impédance (admittance) au circuit, comme à la figure 3.7 [117]. Puisque les équations du mouvement de Hamilton sont invariantes sous renversement du temps, il n'est pas possible d'inclure directement ces éléments dans le traitement présenté aux sections précédentes. L'introduction des processus dissipatifs dans le formalisme hamiltonien a été décrit, en autres, par Feynman et Vernon [127] et Caldeira et Leggett [128]. Plusieurs articles de revues [129,130] et livre [124] décrivent en détail ces approches.

Tel que présenté à la figure 3.8, dans le modèle de Caldeira et Leggett, impédances et admittances sont modélisées par une infinité de circuits $L C$. L'environnement est donc traité comme un bain d'oscillateurs harmoniques couplés au système. Chaque 


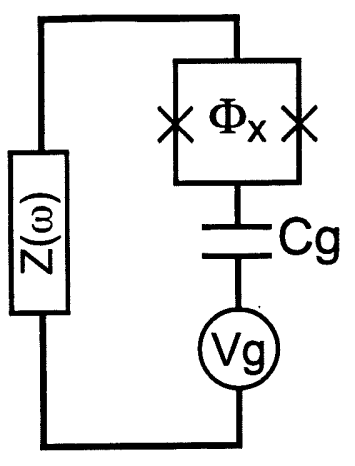

(a)

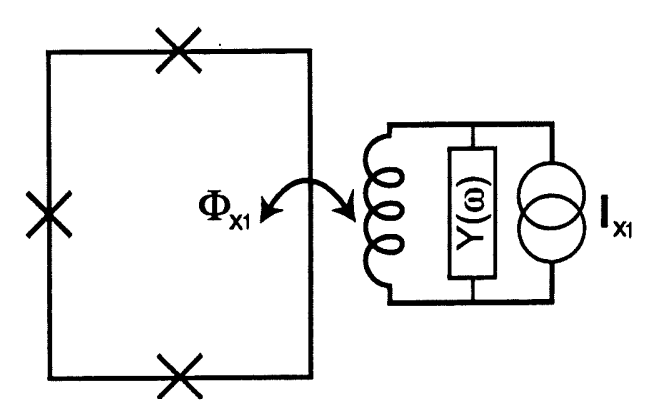

(b)

FIG. 3.7: a) L'effet des fils et des sources de voltages imparfaites peut être modélisé par une impédance $Z(\omega)$ ayant des fluctuations de voltage intrinsèques décrites par un spectre de bruit du type Nyquist-Jonhson. b) Une admittance $Y(\omega)=1 / Z(\omega)$ est utilisée pour modéliser une source de courant imparfaite. Dans les cas a) et b), le bruit se couple à l'opérateur $\sigma_{z}$ du qubit. Le bruit se couplant à $\sigma_{x}$ peut aussi être pris en considération. Par exemple, pour le qubit de charge, cela correspond au bruit dans le courant générant le flux $\delta \Phi_{x}$. Pour le qubit de phase, ceci correspond au bruit du flux $\delta \Phi_{x 2}$ dans la seconde boucle, figure 3.5. Les fluctuations selon $z$ et $x$ sont indépendantes et le qubit est alors couplé à deux environnements indépendants.

oscillateur n'est que faiblement couplé et il est possible de se limiter à un terme d'interaction système-bain linéaire. Quoique chacun ne soit que faiblement couplé, l'effet global de l'environnement peut être très important puisqu'il y a un grand nombre de modes.

Un couplage linéaire entre les modes du bain et le qubit conduit au modèle spinboson. Dans ce modèle, l'hamiltonien total prend la forme $[108,124]$

$$
H=H_{\text {qubit }}+\sum_{i=x, z} \sigma_{i}\left(\sum_{k} \lambda_{k i} x_{k i}\right)+\sum_{k, i}\left(\frac{p_{k i}^{2}}{2 m_{k i}}+\frac{m_{k i} \omega_{k i}^{2} x_{k i}^{2}}{2}\right) .
$$

Le second membre de droite décrit l'interaction système-bain tandis que le dernier est l'hamiltonien du bain. Les modes $k$ sont indépendants et l'opérateur du bain $X_{i}=\sum_{k} \lambda_{k i} x_{k i}$ se couple à l'opérateur $\sigma_{i}$ du qubit.

Dans ce modèle, il est simple d'évaluer les taux de relaxation et de déphasage. 


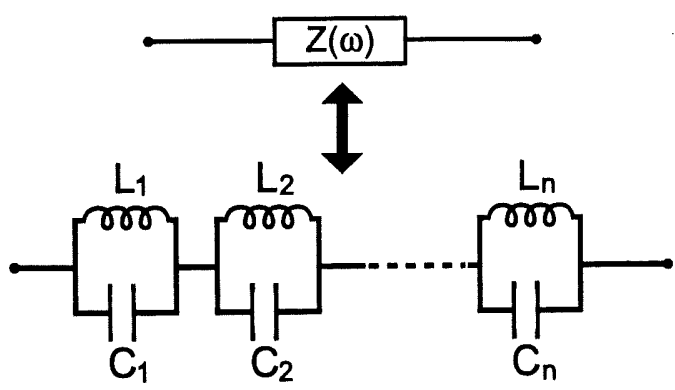

(a)

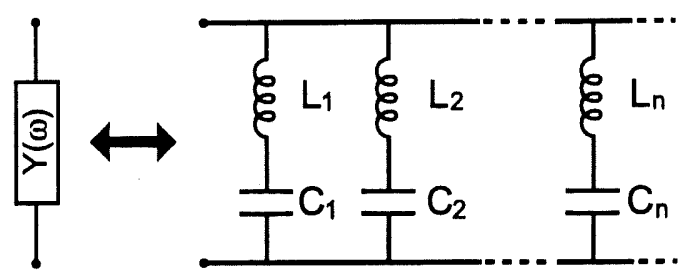

(b)

FIG. 3.8: Modèle de Caldeira-Leggett pour a) une impédance et b) une admittance.

Ceux-ci ont été obtenus, à l'origine, par intégrales de chemin [130,131]. On peut aussi les obtenir à l'aide de techniques diagrammatiques à la Keldysh [132,133]. Toutefois, l'approche la plus simple est probablement d'utiliser la règle d'or de Fermi $[119,134]$. Le calcul ce fait facilement dans la base où l'hamiltonien à un qubit est diagonal $H_{\text {qubit }}=-\Delta E \tau_{z} / 2$. Ici, les $\tau_{i}$ sont les matrices de Pauli dans la base des états propres de $H_{\text {qubit }}$. Dans cette base, l'hamiltonien système-bain peut s'écrire sous la forme $H_{S B}=\eta_{z} f_{z}(t) \tau_{z}+\eta_{x} f_{x}(t) \tau_{x}$, où les $f_{i}(t)$ sont les opérateurs du bain et représentent les fluctuations des champs effectifs sentis par le qubit. Dans ce cas on obtient, au premier ordre en théorie de perturbation, les taux de relaxation et de déphasage suivants :

$$
\begin{aligned}
\Gamma_{r} & =\frac{\eta_{x}^{2}}{\hbar^{2}} S_{f_{x}}(\Delta E / \hbar), \\
\Gamma_{\phi} & =\frac{\Gamma_{r}}{2}+\frac{\eta_{z}^{2}}{\hbar^{2}} S_{f_{z}}(\omega \rightarrow 0) .
\end{aligned}
$$

Ces résultats sont obtenues à l'annexe E. Dans ces expressions, $S_{f_{i}}(\omega)$ est le spectre symétrique de fluctuation de $f_{i}(t)$. À l'équilibre thermique, le théorème fluctuationdissipation donne donc $[108,134]$ :

$$
S_{f_{i}}(\omega)=\left\langle\left\{f_{i}(t), f_{i}\left(t^{\prime}\right)\right\}\right\rangle_{\omega}=2 J_{i}(\omega) \operatorname{coth} \frac{\hbar \omega}{2 k_{B} T},
$$

où $J_{i}(\omega)$ est la densité spectrale du bain [124].

On peut comprendre les résultats (3.31) et (3.32) de la façon suivante. Le bruit à la fréquence $\Delta E / \hbar$ induit des transitions entre les états propres du qubit. Le taux 


\subsection{RELAXATION ET DÉPHASAGE}

de relaxation dépend donc de l'intensité du bruit à cette fréquence. Dans certaines situations, l'impédance du circuit peut être modifiée de façon à éloigner de cette fréquence les pics de la densité spectrale. Dans ce cas, l'impédance agit comme un filtre protégeant le qubit de son environnement $[125,135,136]$. Tandis que le bruit à basse fréquence ne peut induire de transitions, il peut toutefois introduire une phase relative aléatoire entre les états propres. Le bruit à basse fréquence participe donc au déphasage. De même, puisque la relaxation perturbe les éléments hors diagonaux de la matrice densité, ce processus participe aussi au déphasage. Le second terme de (3.32) sera noté par la suite $\Gamma_{\phi}^{*}$. Il s'agit du terme décrivant le déphasage pur.

Dans le contexte du calcul quantique, ces résultats sont extrêmement importants. Puisque les algorithmes quantiques reposent sur les concepts de superpositions d'états et d'enchevêtrement, la cohérence de phase doit être maintenue tout au long du calcul. Ainsi, le calcul le plus long que l'on puisse espérer mener à terme sur un ordinateur quantique sera typiquement beaucoup plus court que $\Gamma_{\phi}^{-1}$ (sans correction d'erreurs).

Afin de déterminer la qualité d'un ordinateur quantique, le taux de déphasage en lui-même n'est pas suffisant. Comme il a déjà été présenté à la section 2.1.1, un paramètre plus approprié est le facteur de qualité $Q$ défini comme le ratio du temps de cohérence sur le temps requis pour réaliser une opération logique : $Q \equiv \Gamma_{\phi}^{-1} / t_{\mathrm{op}}$. Ce ratio indique le nombre d'opérations logiques qu'il est possible de réaliser avant de perdre complètement la cohérence de phase. Le lecteur intéressé trouvera à la référence [137] ce facteur de qualité pour différents systèmes. Il est important de remarquer que le 'temps d'opération' change généralement d'une opération élémentaire à l'autre. Par exemple, pour le qubit de charge, l'énergie Josephson génère les rotations selon $x$ tandis que l'énergie de charge est responsable des rotations selon $z$. Puisque $E_{C}>E_{J}$, le temps caractéristique d'une rotation selon un axe ou l'autre ne sera pas le même. Contrairement à ce que l'on voit généralement dans la littérature, afin d'évaluer le potentiel d'un qubit au calcul il semble donc plus approprié d'utiliser le facteur de qualité suivant $Q=\operatorname{Min}\left\{\Gamma_{\phi}^{-1} / t_{\mathrm{op}}^{z}, \Gamma_{\phi}^{-1} / t_{\mathrm{op}}^{x}, \Gamma_{\phi}^{-1} / t_{\mathrm{op}}^{2 q}\right\}$. Dans cette liste, $t_{\mathrm{op}}^{2 q}$ est le temps typique des opérations à deux qubits.

Un commentaire concernant le calcul tolérant aux imperfections s'impose ici. La théorie du calcul tolérant aux imperfections nous apprend que si la probabilité d'erreurs par porte logique est sous un certain seuil, il est alors possible de faire du 
calcul quantique arbitrairement long [138]. Ce seuil varie selon le modèle d'erreurs et les codes correcteurs utilisés mais, il est généralement accepté qu'il sera de l'ordre de $10^{-4}$ à $10^{-5}$ [139]. En lien avec ces résultats, on lit souvent dans la littérature [108] que si le facteur de qualité d'un qubit est supérieur à $10^{4}-10^{5}$, il sera possible d'utiliser les techniques du calcul tolérant aux imperfections. Cette affirmation n'est évidemment pas fondée. En effet, le facteur de qualité nous informe sur le nombre d'opérations logiques que l'on peut espérer réaliser avant que le qubit ne soit complètement déphasé. Tel que mentionné précédemment, afin d'être utile au calcul quantique, ce nombre d'opérations devra typiquement être beaucoup plus petit. De plus, le déphasage du qubit n'est certainement pas la seule source d'erreurs, on pense en particulier aux erreurs de réalisation des opérations logiques, aux imperfections dans l'initialisation et la lecture et à l'effet de la présence des autres qubits.

Finalement, seule la partie électromagnétique de l'environnement du qubit a été considérée dans cette section. D'autres sources de bruit peuvent toutefois être présentes : interaction non voulue entre qubits, bruit en $1 / f$, quasiparticules, spins nucléaires,... Leur effet a été étudié par différents auteurs [108,140-143]. De ces sources, le bruit en $1 / f$ est peut-être le plus dommageable. Évidemment, la partie de l'environnement qui affecte le système le plus fortement dépend du degré de liberté incarnant le qubit.

\subsection{Qubits supraconducteurs : designs et réalisations}

\subsubsection{Qubits basés sur la charge}

Le design original du qubit de charge supraconducteur, étudié aux sections $\S 3.1 .1$ et §3.2.1, a été suggéré par Shnirman et al. [78] puis amélioré par Makhlin et al. [145]. Quelques succès expérimentaux avec la boîte de Cooper ont été présentés dans la littérature. Premièrement, la présence de superposition cohérente d'états de charge au point de dégénérescence a été vérifiée expérimentalement par Bouchiat et al. [146]. Plus récemment, Nakamura et al. ont observé des oscillations cohérentes [144] et de 

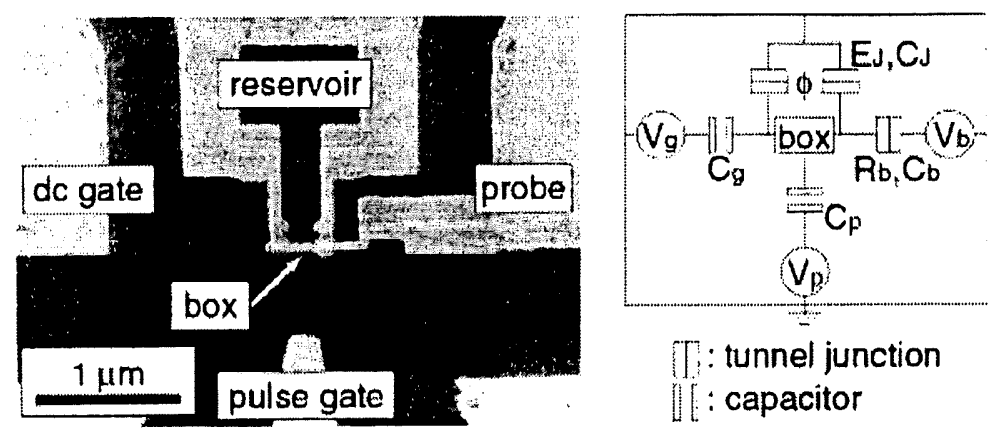

FIG. 3.9: Design utilisé par l'équipe de NEC pour l'observation des oscillations cohérentes entre états de charge dans une boîte de Cooper. Comme à la figure 3.3, l'état de la boîte peut être contrôlé à l'aide du flux extérieur (appliqué dans la boucle notée 'reservoir' sur la figure) et du voltage de grille (appliqué par les électrodes 'dc gate' et 'pulse gate'). L'électrode notée 'probe' réalise une mesure faible de façon continue de l'état de charge. Figure tirée de [144].

Rabi [147] de la charge avec le design présenté à la figure $3.9^{2}$. La différence principale entre ce circuit et celui de la figure 3.3 est la présence de l'électrode notée 'probe'. Le courant de quasiparticules dans cette jonction dépend de l'état de charge de la boîte. La mesure de ce courant constitue donc une mesure du qubit. À l'aide de ce design, les oscillations cohérentes ont pu être observées sur environ $5 \mathrm{~ns}$. Le temps de cohérence est probablement limité par la jonction de lecture qui effectue une mesure continue du qubit. Dans une expérience plus récente, ce même qubit de charge a été soumis à des séquences d'impulsions du type écho de spin [148]. Les résultats de cette étude suggèrent que le bruit en $1 / f$ à basse fréquence soit la source dominante de déphasage dans ce système. Encore plus récemment ce même groupe a réussi à opérer dans le régime quantique une paire de qubits de charge couplés capacitivement et ainsi montrer l'évidence d'enchevêtrement entre ceux-ci [149].

\footnotetext{
${ }^{2}$ Comme il se doit, on fait la distinction entre oscillations de Rabi et oscillations cohérentes. Dans le premier cas, les oscillations sont entretenues par une perturbation extérieure résonante. Dans le second cas, les oscillations sont spontanées. Dans la situation qui nous intéresse ici, elle se manifeste (en particulier) au point de dégénérescence où les états propres du qubit sont des superpositions de charge : $(|0\rangle+|1\rangle) / \sqrt{2}$. Un système initialement préparé dans l'état $|0\rangle$ oscillera alors spontanément entre les états $|0\rangle$ et $|1\rangle$.
} 


\subsection{QUBITS SUPRACONDUCTEURS : DESIGNS ET RÉALISATIONS}

Notons qu'un design de qubit de charge alternatif a été suggéré par Averin [150]. Dans ce cas, chaque qubit requiert un grand nombre de jonctions et de voltages de grille. Cette complexité additionnelle rend la réalisation et l'utilisation de ce qubit difficile:

\subsubsection{Qubits basés sur la phase}

Les qubits de flux ont été introduits par Bocko et al. [151]. Leur suggestion est basée sur le SQUID-rf à une jonction. Ce système a un comportement similaire au design à trois jonctions décrit précédemment. Celui-ci n'a toutefois qu'une différence de phase et donc qu'une seule variable dynamique : le flux total dans la boucle. Malheureusement, la bistabilité dans ce cas requiert une grande inductance $L>\Phi_{0}^{2} / 4 \pi^{2} E_{J}$ [108]. Les flux générés par les courants horaire et anti-horaire correspondant aux états logiques du qubit seront alors importants $\left(\sim \Phi_{0}\right)$ et le qubit sera fortement couplé à son environnement. On ne peut donc s'attendre à maintenir la cohérence de phase suffisamment longtemps pour réaliser un calcul utile avec ce design. Mentionnons aussi que l'article original de Bocko et al. contient beaucoup de problèmes du point de vue de l'informatique quantique. Par exemple, ceux-ci décrivent une méthode pour copier l'état d'un qubit vers un second qubit. Ceci est évidemment impossible à réaliser pour des états arbitraires $[152,153]$.

Quoique ce système ne semble pas utile pour réaliser un qubit, plusieurs aspects du comportement quantique des SQUIDs-rf ont été vérifiés expérimentalement. Par exemple, le groupe de Stony Brook a observé l'effet tunnel résonant entre les états des puits du SQUID [154]. Plus récemment, ce même groupe a observé indirectement la présence de superposition cohérente d'états excités des deux puits [155]. Pour cette dernière observation, l'inductance de la boucle du SQUID était de $240 \mathrm{pH}$ ( $E_{L} \equiv$ $\left.\Phi_{0}^{2} / 2 L \sim 645 \mathrm{~K}\right)$, l'énergie Josephson $E_{J} \sim 76 \mathrm{~K}$ et l'énergie de charge $E_{C} \sim 9 \mathrm{mK}$. Comme il se doit, $E_{L}, E_{J} \gg E_{C}$. Les états participant à la superposition diffèrent par un flux d'environ $\Phi_{0} / 4$. Il s'agit donc d'une superposition d'états 'macroscopiques'.

À l'opposé du SQUID-rf, l'avantage du qubit de phase à trois jonctions est que la bistabilité est possible même pour les petites inductances. Il est donc possible d'avoir des flux beaucoup plus faibles réduisant du même coup le couplage à l'environnement. 


\subsection{QUBITS SUPRACONDUCTEURS : DESIGNS ET RÉALISATIONS}

Presque simultanément à l'expérience du groupe de Stony Brook, l'évidence de superposition des états fondamentaux du double puits dans le design à trois jonctions a été présentée par la collaboration Delft-MIT [156]. Dans ce cas, l'inductance de la boucle était de $\sim 5 \mathrm{pH}$, soit beaucoup plus petite que pour l'expérience basée sur le SQUID-rf. De plus, l'énergie Josephson était $\sim 2 \mathrm{~K}$ avec $\alpha \sim 0.75$ (voir figure 3.5) et le ratio avec l'énergie de charge $E_{J} / E_{C} \sim 80$. Pour ces paramètres, les états participant à la superposition ont une différence de flux d'environ $10^{-3} \Phi_{0}$. Malgré que cette différence soit beaucoup plus petite que dans le cas de l'expérience de Stony Brook, il est intéressant de remarquer que ces flux correspondent à quelques millions de paires de Cooper circulant simultanément dans les directions horaire et anti-horaire [156]. Il s'agit donc bien d'une superposition cohérente d'états 'macroscopiques'. Ce design peut être amélioré en utilisant une configuration de type 'gradiomètre' [157].

Une étude récente montre comment il est possible d'optimiser les performances du qubit basé sur le SQUID-rf à une jonction en utilisant, lors des opérations logiques, un troisième niveau [158]. Évidemment, la précision temporelle des opérations logiques est alors particulièrement cruciale afin de ne pas peupler involontairement ce niveau additionnel. Ce type d'approche semble avoir certaines similitudes avec le mode d'opération des architectures basées sur les pièges linéaires à ions [159]. Puisque les SQUID-rfs ne feront vraisemblablement pas de bons qubits, il serait intéressant d'appliquer ce type d'optimisation aux autres qubits de phase.

Les qubits présentés jusqu'à présent sont basés sur les supraconducteurs dont le paramètre d'ordre est de symétrie $s$. Il existe aussi des architectures basées sur les supraconducteurs dont le paramètre d'ordre est de symétrie $d[71,160]$ et sur la combinaison de types $s$ et $d[161,162]$. Le lecteur intéressé pourra consulter le court article de revue sur le sujet [163].

Quoiqu'ils soient plus difficiles à fabriquer, ces designs basés sur les supraconducteurs de type $d$ ont certains avantages. En particulier, la bistabilité des qubits de type $s$ n'est possible qu'en présence d'un flux extérieur $\left(\sim \Phi_{0} / 2\right)$. Ainsi, le simple fait de garder inactif un qubit $(\varepsilon=0, \Delta=0)$ nécessite l'application de flux externes. Ces qubits sont très sensibles aux fluctuations de flux et accumuleront rapidement une phase relative aléatoire [161]. À l'opposé, la symétrie plus faible du paramètre d'ordre des cuprates permet d'avoir la bistabilité même sans flux externe, réduisant 


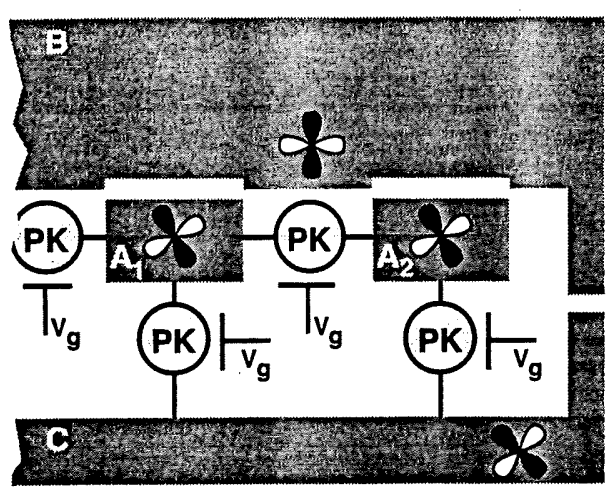

(a)

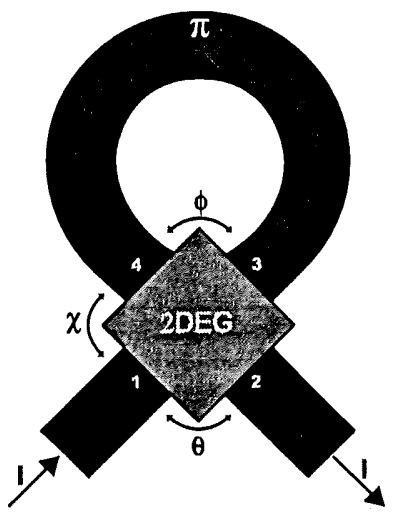

(b)

FIG. 3.10: a) Qubits basés sur les 'barrières de grain' entre supraconducteurs de type $d$. Les terminaux $\mathrm{A}_{1}, \mathrm{~A}_{2}$ et $\mathrm{B}$ sont formés de supraconducteurs de type $d$, les $\mathrm{PK}$ (parity key) représentent des SSETs. Les lobes positifs du paramètre d'ordre sont en gris foncé. Le terminal $\mathrm{C}$ agit comme un qubit ayant un état logique fixe. En plus de ce bus, deux qubits sont présentés. b) Qubit à terminaux multiples. Des supraconducteurs de type $s$ sont connectés à un 2DEG. L'élément de type $d$ dans ce design est la jonction $\pi$. Celle-ci a le même effet qu'un flux extérieur de $\Phi_{0} / 2$.

ainsi les sources d'erreurs. Par exemple, on peut fabriquer une jonction $\pi$ à l'aide d'un supraconducteur de type $d$ en se connectant à angle droit sur celui-ci [164]. Les jonctions $\pi$ sont particulièrement intéressantes car elles peuvent jouer le rôle d'un flux externe $\Phi_{0} / 2$. De plus, comme pour les qubits à trois jonctions, le flux correspondant aux états logiques pour ces qubit de type $d$ est très faible $\left(\ll \Phi_{0}\right)$, réduisant le couplage à l'environnement. Un troisième avantage est que, puisque le flux n'est pas nécessaire pour avoir bistabilité, il est possible de contrôler ces qubits électriquement, sans flux extérieur $[52,161,162]$. Cette approche semble avantageuse puisque le flux appliqué sur un qubit donné, pourra être ressenti par ses voisins si l'écrantage n'est pas approprié.

La figure 3.10 présente deux architectures de qubit de type $d$ ne requérant pas de flux externe pour les opérations. Le design basé sur les barrières de grain entre supraconducteurs de type $d$ [165] a été suggéré par Zagoskin [160]. La réalisation 
d'opérations logiques sur ce design a été étudiée par Blais et Zagoskin [67] et par Lidar et al. [52]. Cette dernière publication est reproduite à l'annexe C. Dans le design étudié par ces derniers auteurs et présenté à la figure 3.10 a), les opérations logiques sans flux extérieur sont rendues possibles par l'utilisation d'un 'bus qubit' (terminal C) et du concept d'universalité encodé déjà abordée à la section 1.5.3. Notons que la bistabilité d'une jonction basée sur les barrières de grain entre supraconducteurs de type $d$ a récemment été montrée expérimentalement [166].

L'architecture de la figure $3.10 \mathrm{~b}$ ) utilise trois terminaux de type $s$ connectés à un gaz électronique bi-dimensionel (2D electron gas, 2DEG). Ce type de jonctions S-2DEG-S a été étudié expérimentalement par Heida et al. [167]. Dans ce design, une jonction $\pi$ est insérée dans la boucle. Cette jonction joue le rôle d'un flux extérieur $\Phi_{0} / 2$ assurant la bistabilité sans toutefois introduire de fluctuations supplémentaires. Les opérations logiques sont réalisées en variant le courant de transport $I$. Plus de détails sur cette architecture se trouve à la référence [162] reproduite en annexe D.

\subsubsection{Designs récents}

Récemment, des résultats expérimentaux sur des designs n'appartenant pas aux catégories de qubits présentées jusqu'à présent sont parus. Premièrement, le groupe de Saclay a proposé [126] et testé avec succès [6] l'architecture présentée à la figure 3.11 a). Pour ce circuit, $E_{J} \approx E_{C}$ de sorte que ni la phase ni la charge ne sont de bons nombres quantiques, comme on le voit clairement aux figures $3.11 \mathrm{~b}$ ) et c). Au voltage de grille $n_{g}=1 / 2$, les deux premiers états propres, notés $|0\rangle$ et $|1\rangle$, sont des superpositions symétrique et antisymétrique de charges. Ces états sont tels que la charge moyenne est la même dans les deux cas : $\langle 0|\hat{n}| 0\rangle=\langle 1|\hat{n}| 1\rangle=1 / 2$. De plus, la séparation en énergie entre ces deux premiers états propres et les états suivants n'est pas la même. Ainsi, si le facteur de qualité du système est grand (la séparation entre les niveaux d'énergie est beaucoup plus importante que la largeur de ces niveaux) $|0\rangle$ et $|1\rangle$ peuvent être utilisés comme états logiques.

Un avantage de ce choix d'état logique est que puisque $\langle\hat{n}\rangle_{0,1}=1 / 2$ ces deux états présentent la même charge moyenne à l'environnement, réduisant ainsi la décohérence. Un second avantage de ce design est qu'au point de dégénérescence $n_{g}=1 / 2, \partial \Delta E / \partial n_{g}$ 


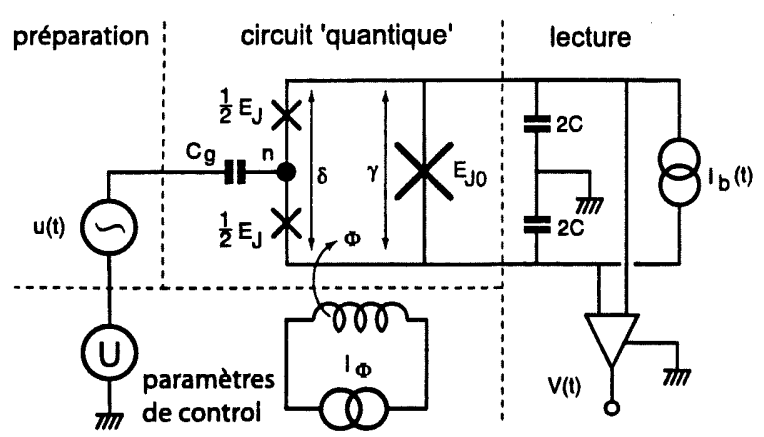

(a)

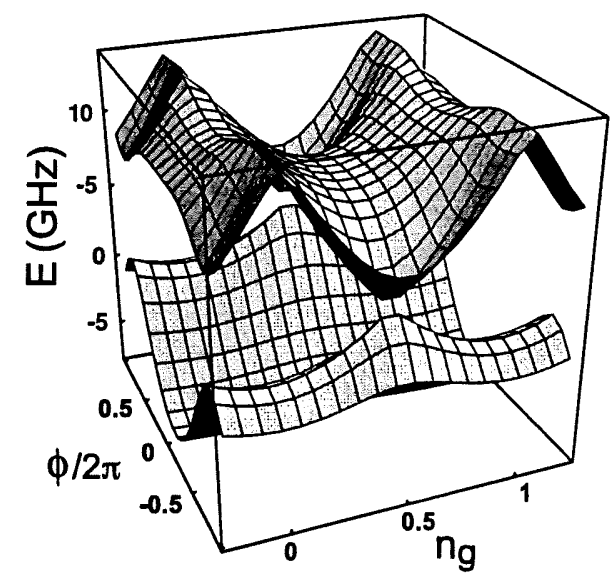

(c)

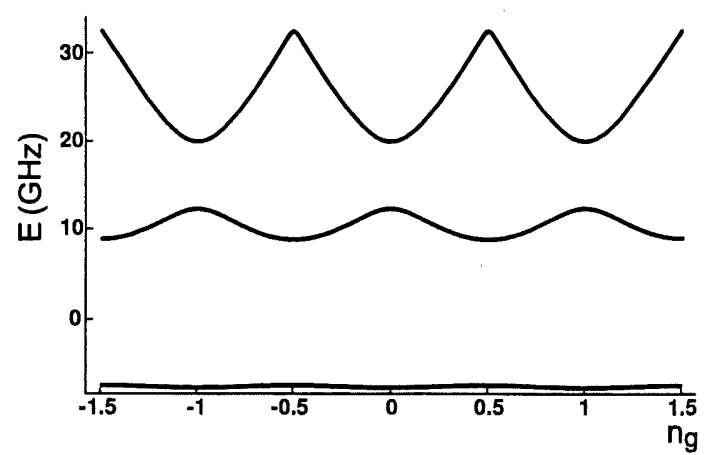

(b)

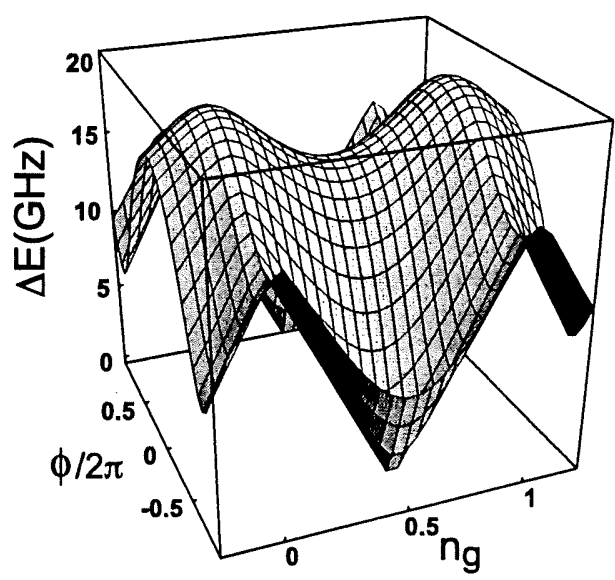

(d)

FIG. 3.11: a) Circuit électrique pour le qubit phase-charge de Saclay. Figure adaptée de la référence [6]. b) Spectre énergétique en fonction de la charge de grille et à flux extérieur nul. Les énergies sont ici $E_{J}=0.86 k_{B} \mathrm{~K}$ et $E_{J} / E_{C}=1.27$ comme dans l'expérience décrite à la référence [6]. Les deux premiers niveaux jouent le rôle d'états logiques. c) Spectre des états logiques en fonction de la charge de grille et du flux extérieur $\phi=\Phi / \Phi_{0}$. d) Différence d'énergie entre les états logiques en fonction du voltage de grille et du flux extérieur. Le point d'opération $\left(n_{g}=1 / 2, \phi=0\right)$ est un point de selle. À ce point le système n'est donc pas sensible, au premier ordre, aux fluctuations des paramètres de contrôle. La lecture du qubit est réalisée en appliquant un courant $I_{b}$. On mesure alors un voltage $V(t)$ selon l'état logique du qubit. Plus de détails concernant la mesure seront donnés au chapitre 5. 


\subsection{QUBITS SUPRACONDUCTEURS : DESIGNS ET RÉALISATIONS}

$=0$ où $\Delta E$ est la différence en énergie entre les états logiques illustrée figure $3.11 \mathrm{~d}$ ). De plus, si le flux extérieur $\Phi$ est nul, $\Delta E$ est telle que $\partial \Delta E / \partial \Phi=0$. Ainsi, en maintenant le qubit au point d'opération $\left(n_{g}=1 / 2, \Phi=0\right)$, celui-ci n'est pas sensible au premier ordre aux fluctuations de voltage et de flux.

Afin de minimiser l'effet du bruit sur le système, ces paramètres sont gardés au point d'opération au cours des manipulations cohérentes et ne peuvent être utilisés pour réaliser les opérations logiques. Les rotations selon $x$ et $y$ sont plutôt réalisées en appliquant un voltage de grille alternatif à la fréquence $\Delta E / \hbar$.

À l'aide de ce design phase-charge ingénieux, le groupe de Saclay a pu observer des oscillations de Rabi ainsi que des franges de Ramsey [168]. Les temps de relaxation et de déphasage extraits de ces observations sont respectivement $T_{1} \sim 1.8 \mu$ s et $T_{2} \sim$ $0.5 \mu \mathrm{s}$. Notons que la prédiction théorique pour le temps de relaxation est de $\sim 10 \mu \mathrm{s}$ [6]. Un écart de seulement un ordre de grandeur pour une première expérience sur ce système est très encourageant pour l'avenir.

Un second type d'expériences impliquant une seule jonction Josephson de grande dimension $\left(\sim 10 \times 10 \mu \mathrm{m}^{2}\right)$ et à travers laquelle est appliqué un courant continu (current-biased Josephson-Junction, CBJJ) a été réalisée indépendamment par Martinis et al. [7] et par Yu et al. [8], figure 3.12 a).

Le potentiel vu par la phase d'une CBJJ a la forme bien connue d'une planche à laver (washboard potential), figure $3.12 \mathrm{~b}$ ). Si le courant appliqué $I_{b}$ s'approche du courant critique de la jonction, chacun des puits du potentiel n'a que quelques niveaux. Dans l'expérience décrite à la référence [7], le courant est ajusté de sorte qu'il n'y ait que trois niveaux par puits. Les deux premiers niveaux de l'un des puits jouent le rôle de sous-espace logique $\{|0\rangle,|1\rangle\}$. Le troisième niveau, $|2\rangle$, est très près du maximum de la barrière de potentiel. La probabilité tunnel vers le continuum est grande et le temps de vie de cet état est par conséquent très court. Les opérations logiques sont réalisées en appliquant un courant alternatif à la fréquence de transition $\omega_{01}$ entre les états logiques. Comme dans le cas du qubit de Saclay, une phase relative est toujours accumulée et devra être refocalisée. Ici toutefois, la fréquence de transition $\omega_{01}$ peut être ajustée en variant l'amplitude du courant continu. La mesure est réalisée en pompant premièrement le système à la fréquence de transition $\omega_{12}$ entre les états $|1\rangle$ et $|2\rangle$. Si, suite à l'application de ce courant alternatif, un voltage apparaît aux 


\subsection{QUBITS SUPRACONDUCTEURS : DESIGNS ET RÉALISATIONS}

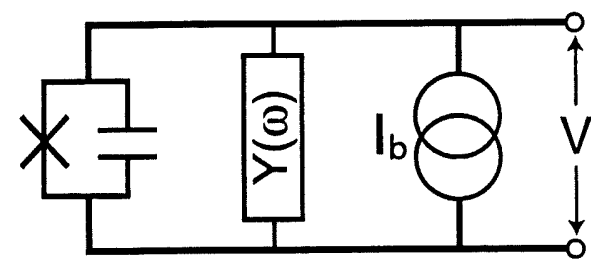

(a)

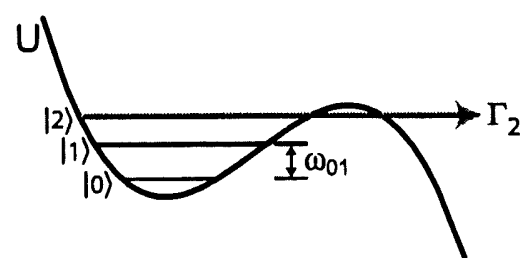

(b)

FIG. 3.12: a) Jonction Josephson sur laquelle on applique un courant (Current biased Josephson-jonction, CBJJ). Dans les expériences décrites aux références [7] et [8], les jonctions ont une grande capacité, $\sim 6 \mathrm{pF}$. La clé du succès de ces expériences est de manipuler l'impédance de l'environnement de façon à ce que l'impédance effective vue par le qubit soit grande et donc les fluctuations de courant faibles. Par exemple, dans l'expérience de Martinis et al., $1 / \operatorname{Re}[Y(\omega)] \sim 560 \mathrm{k} \Omega$, ce qui est beaucoup plus grand que la valeur typique de $100 \Omega$ aux fréquences micro-ondes [118]. Plus de détails concernant l'importance de l'admittance sur la dynamique d'une CBJJ seront présentés à la section 5.3.1. b) Potentiel en forme de planche à laver avec seulement quelques niveaux dans chaque puits. Un seul puits est présenté. Les deux premiers niveaux forment le sous-espace de calcul. Le taux tunnel $\Gamma_{2}$ de l'état $|2\rangle$ vers le continuum est très grand. Figures adaptées de la référence [7].

bornes de la jonction, le résultat de la mesure est $|1\rangle$. Ce type de qubit sera décrit de façon plus détaillée au chapitre 4.

Avec ce système, Martinis et al. ont pu observer des oscillations de Rabi en fonction de la puissance du courant alternatif. Le temps de déphasage extrait de ces observations est $\sim 10 \mathrm{~ns}$. Un meilleur choix de matériaux pourrait améliorer ce temps [7]. L'expérience décrite à la référence [8] utilise un design similaire mais où plus de niveaux sont présents dans chaque puits. Dans ce cas, les oscillations de Rabi en fonction du temps ont été observées. Le temps de déphasage est estimé à $4.9 \mu$ s.

Un avantage de cette architecture est qu'il ne requiert qu'une jonction par qubit. Il est toutefois essentiel d'écranter les fluctuations de courant en manipulant l'impédance de l'environnement de façon à ce que sa partie réelle soit grande (le bruit de courant Johnson-Nyquist sur la jonction va comme $1 / \operatorname{Re}[\mathrm{Z}(\omega)])$. Ceci a été réalisé différemment dans chacune des expériences citées ici. Notons finalement que puisque 
le troisième niveaux est fortement couplé au continuum, il est particulièrement important de considérer la 'fuite hors du sous-espace de calcul' [169] dans ce système. Cette question est abordée en plus grands détails au chapitre 4.

\subsection{Sommaire}

Dans ce chapitre, plusieurs architectures de qubits supraconducteurs ont été présentées. La compétition entre énergie de charge et énergie Josephson donne une grande richesse à la dynamique des Jonctions Josephson et rend possible un grand nombre de qubits supraconducteurs différents. Chaque design a ses avantages et inconvénients. En raison de l'existence d'un point d'opération où les états logiques sont insensibles aux fluctuations, le qubit phase-charge semble particulièrement intéressant. Ce type de point particulier n'est toutefois certainement pas limité à ce design de qubit et la recherche de nouveaux designs devrait être guidée par ce type de considération.

Afin de ne pas alourdir la discussion, certaines des contributions originales de ce travail en lien avec les qubits supraconducteurs n'ont été que brièvement présentées dans les pages précédentes. Ces contributions sont décrites en détails aux annexes $\mathrm{C}$ et $\mathrm{D}$. Rappelons qu'à l'annexe $\mathrm{C}$, on s'intéresse au concept d'universalité encodé appliqué aux qubits de phase. Il s'agit, à notre connaissance, de la première étude sur le sujet. À l'annexe D, on décrit un nouveau design de qubit supraconducteur. Celui-ci utilise une jonction $\pi$ dans le but d'atteindre la bistabilité sans flux extérieur. Une autre contribution originale brièvement abordée ici concerne les qubits basés sur les barrières de grain entre supraconducteurs de type $d$. Ces travaux ont été décrit en détails dans un autre contexte $[4,5]$, et ne sont pas répétés ici. 


\section{Chapitre 4}

\section{Interaction qubit-qubit ajustable}

Best possible knowledge of a whole does not include best possible knowledge of its parts - and this is what keeps coming back to haunt us.

- Erwin Schrödinger

Dans le chapitre précédent, plusieurs designs de qubits supraconducteurs ont été exposés ainsi que certains des succès expérimentaux les plus marquants. Étant donné la qualité des résultats obtenus à l'aide des designs du type phase-charge et du type CBJJ, le prochain défi expérimental semble être de montrer l'enchevêtrement d'une paire de qubits.

Dans ce chapitre, on s'intéresse à une façon originale de faire interagir différents types de qubits supraconducteurs. On commence par rappeler différentes suggestions parues dans la littérature.

\subsection{Méthodes d'interaction pour qubits supraconducteurs}

\subsubsection{Qubits de charge}

Pour les qubits de charge, plusieurs méthodes d'interaction ont été suggérées. La technique la plus simple est probablement de connecter l'île des deux qubits à l'aide d'une capacité [83] ou d'une jonction Josephson [170]. Dans le premier cas, la charge 


\subsection{MÉTHODES D'INTERACTION POUR QUBITS SUPRACONDUCTEURS}

sur l'île d'un qubit influence la charge sur l'île de son voisin de la même façon que le fait le voltage de grille $V_{g}$. Ceci se traduit dans l'hamiltonien par un terme de la forme $n_{1} n_{2} / \tilde{C}_{\text {int }}$, où $n_{i}$ est la charge sur l'île du qubit $i$ et où $\tilde{C}_{\text {int }}$ dépend de la capacité reliant les qubits. Grâce au remplacement (3.23) vers les matrices de Pauli, on trouve une interaction ayant la forme $\sigma_{z} \sigma_{z}$ dans la base de calcul. Comme on l'a vu à la section $\S 1.4$, une telle interaction permet de réaliser l'opération $C_{P}$ qui est équivalente au non-contrôlé. Ce type d'interaction capacitive a récemment été réalisée expérimentalement avec succès [149]. En raison de la capacité intrinsèque de la jonction, on a aussi le terme d'interaction $\sigma_{z} \sigma_{z}$ avec une jonction Josephson. L'énergie Josephson introduit également un terme $E_{J} \cos \left(\phi_{1}-\phi_{2}\right)$, où $\phi_{i}$ est la phase conjuguée à $n_{i}$. Avec le remplacement (3.23), ceci se traduit par une interaction ayant la forme $\sigma_{x} \sigma_{x}+\sigma_{y} \sigma_{y}$ dans la base de calcul. Cette interaction permet aussi de réaliser le $\mathrm{C}_{\text {NOT }}$.

Un problème évident relié à ce type d'interaction entre qubits est que l'on a aucun contrôle dynamique sur l'amplitude du couplage entre les qubits puisque celle-ci est fixée au moment de la fabrication. L'interaction est par conséquent toujours en opération. Il est toutefois possible d'utiliser les techniques de refocalisation pour annuler, en moyenne, l'interaction lorsqu'elle n'est pas nécessaire au calcul. Par exemple, la refocalisation d'une interaction $J \sigma_{z} \sigma_{z}$ est basée sur les identités $\sigma_{x} \sigma_{z} \sigma_{x}=-\sigma_{z}$ et $\sigma_{x}^{2}=\mathbb{I}$. Ainsi, suite à l'évolution libre sous l'hamiltonien $J \sigma_{z} \sigma_{z}$ sur une période $\tau / 2$, si on applique sur un des qubits une paire de pulses $\pi$ séparées d'une seconde période $\tau / 2$ d'évolution libre, l'action de l'interaction sur $\tau$ est complètement annulée :

$$
R_{x 1}(\pi) e^{-i J \tau \sigma_{z 1} \sigma_{z 2} / 2} R_{x 1}(\pi) e^{-i J \tau \sigma_{z 1} \sigma_{z 2} / 2}=e^{+i J \tau \sigma_{z 1} \sigma_{z 2} / 2} e^{-i J \tau \sigma_{z 1} \sigma_{z 2} / 2}=\mathbb{I}
$$

Ceci complique toutefois. les manipulations et une refocalisation imparfaite entraîne des erreurs. De même, ces techniques de refocalisation sont bien adaptées à la résonance magnétique nucléaire où les interactions spin-spin sont plutôt lentes $(10-100 \mathrm{~Hz})$. Dans les systèmes supraconducteurs, les énergies sont typiquement plus grandes et l'interaction plus rapide $(10 \mathrm{MHz}-10 \mathrm{GHz})$. Ceci a comme avantage que la vitesse d'horloge de l'ordinateur quantique est supérieure. En contrepartie, ceci signifie que les impulsions de refocalisation doivent être rapides et précises, ce qui augmente davantage les complications. De même, le seuil (threshold) de probabilité d'erreurs pour 


\subsection{MÉTHODES D'INTERACTION POUR QUBITS SUPRACONDUCTEURS}

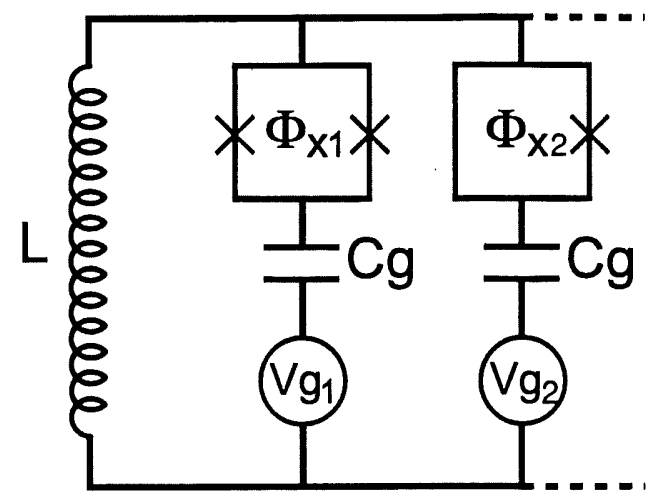

FIG. 4.1: Qubits couplés en parallèle à une inductance $L$.

le calcul tolérant aux imperfections n'a pas encore été évalué dans le cas où l'interaction qubit-qubit est toujours présente. Il est à espérer que ce seuil n'est pas trop bas, ce qui rendrait ce type d'approche impossible à utiliser en pratique.

Notons qu'en remplaçant la jonction reliant les qubits par un SQUID, il est possible de contrôler l'amplitude du couplage à l'aide d'un flux externe [171]. En pratique toutefois, les jonctions formant le SQUID ne seront pas identiques et une interaction résiduelle sera toujours présente.

Une troisième approche, mise de l'avant par le groupe de G. Schön, utilise une inductance connectée en parallèle avec les qubits, figure $4.1[78,108]$. Dans cette approche, l'inductance est choisie de façon à ce que la fréquence $\omega_{L C}=1 / \sqrt{N C_{q b} L}$ de l'oscillateur associé soit plus grande que les énergies typiques des qubits $\left(E_{J}\right.$ et $\left.E_{C}\right)$. Ici, $C_{q b}$ est la capacité effective d'un qubit $C_{q b}^{-1}=1 / C_{g}+1 / C_{J}$ et $N$ est le nombre de qubits connectés à l'inductance. Dans ce cas, les qubits ne peuvent exciter l'oscillateur $L C$ et c'est plutôt grâce aux excitations virtuelles de celui-ci que le couplage qubitqubit est possible.

En écrivant l'hamiltonien du système qubits + oscillateur puis en éliminant les variables de ce dernier on trouve l'interaction qubit-qubit effective suivante $[78,108]$

$$
H_{\mathrm{int}}=-\sum_{i<j} \frac{B_{x}\left(\Phi_{x i}\right) B_{x}\left(\Phi_{x j}\right)}{E_{L}} \sigma_{y i} \sigma_{y j}
$$

avec $E_{L}=\left(C_{J} / C_{q b}\right)^{2} \Phi_{0}^{2} / \pi^{2} L$ et $B_{x}\left(\Phi_{x i}\right)=2 E_{J} \cos \left(\pi \Phi_{x i} / \Phi_{0}\right)$. Cette interaction sera présente dans la mesure où les fluctuations de la phase $\Phi$ du circuit LC sont faibles : 


\subsection{MÉTHODES D'INTERACTION POUR QUBITS SUPRACONDUCTEURS}

$\sqrt{\left\langle\Phi^{2}\right\rangle} \ll C_{J} \Phi_{0} / C_{q b}$

Cette approche a comme avantage que l'interaction entre les qubits $i$ et $j$ (où $i$ et $j$ ne sont pas nécessairement voisins) peut être fermée en ajustant $B_{x}\left(\Phi_{x i}\right)$ ou $B_{x}\left(\Phi_{x j}\right)$ à zéro. Toutefois, cet avantage implique en contrepartie plusieurs problèmes. En effet, on remarque premièrement qu'il est impossible de faire des rotations simultanément selon $x$ sur des qubits différents sans faire interagir ceux-ci avec le terme $H_{\text {int }}$. Il est par conséquent impossible de faire des opérations en parallèle (sauf les rotations à un qubit selon $z$ ) avec ce design. On ne peut donc tirer avantage des optimisations discutées à la section $\S 1.7$. Ce qui est plus grave est que les techniques de calcul quantique tolérant aux imperfections nécessitent un haut degré de parallélisme classique [172]. Un ordinateur quantique construit avec cette approche pour l'interaction qubit-qubit ne pourra donc pas atteindre le seuil de tolérance du calcul tolérant aux imperfections.

Un problème encore plus important concernant cette approche est que, afin d'avoir une interaction suffisamment importante et ainsi des opérations à deux qubits rapides, l'inductance doit être $L \geq 1 \mu \mathrm{H}$ [108]. Un design légèrement différent permet plutôt de prendre $L \gtrsim 1 \mathrm{nH}$ [173]. Sachant qu'une inductance microfabriquée standard a une inductance par unité de longueur d'environ $\sim 1 \mathrm{pH} / \mu \mathrm{m}$ [174], il apparaît clairement que l'inductance nécessaire pour réaliser l'interaction entre qubits médiée par les excitations virtuelles d'un oscillateur LC serait difficile à fabriquer et à intégrer en pratique. Une approche récente et légèrement différente règle, en principe, le problème d'intégration mais souffre toujours de l'impossibilité de faire des opérations logiques en parallèle [175].

Finalement, une technique alternative utilise le couplage inductif mutuel entre les SQUIDs formant les boucles des qubits de charge [176]. Puisque la self de ces boucles est en pratique très faible, il semble que ce couplage inductif soit petit et donc que les opérations résultantes soient lentes.

\subsubsection{Qubits de phase}

Pour les qubits de phase du type à trois jonctions ou SQUID-rf, l'interaction qui semble la plus naturelle est le couplage inductif. En plaçant les boucles de deux qubits à proximité, on obtient un terme $M I_{1} I_{2}$ dans l'hamiltonien, avec $M$ l'inductance 


\subsection{MÉTHODES D'INTERACTION POUR QUBITS SUPRACONDUCTEURS}

mutuelle et $I_{i}$ le courant dans la boucle du qubit $i$ [121]. Selon que l'on couple ainsi les boucles principales ou secondaires des qubits, on obtient un terme d'interaction ayant la symétrie $\sigma_{z} \sigma_{z}, \sigma_{z} \sigma_{x}$ ou $\sigma_{x} \sigma_{x}$. Les temps caractéristiques pour l'interaction qubit-qubit sont du même ordre que ceux des opérations à un bit [108].

Cette approche est similaire en esprit au couplage capacitif pour les qubits de charge et souffre du même problème : l'interaction qubit-qubit est toujours présente. Une alternative utilise les excitations virtuelles d'un circuit LC couplé inductivement au registre de qubits [173]. Comme on peut s'y attendre, cette approche est équivalente à celle de la figure 4.1 et présente les mêmes complications.

Pour les qubits basés sur les supraconducteurs à haute température critique, différentes approches ont été suggérées. Pour les qubits basés sur les barrières de grain présentés à la figure $3.10 \mathrm{a}$ ), un transistor à 'un électron' supraconducteur (superconducting single electron transistor, SSET) entre les qubits permet une interaction de la forme $\sigma_{z} \sigma_{z}$ [5]. L'amplitude de l'interaction dépend de l'énergie Josephson des jonctions du transistor. Celles-ci peuvent être choisies de telle sorte que le temps caractéristique d'interaction soit du même ordre de grandeur que les opérations à un qubit. De même, en ajustant le voltage de grille du SSET, il est possible de fermer ou d'ouvrir le couplage entre qubits. Les SSETs ont l'avantage de commuter rapidement entre ces états ouvert et fermé (qubits découplés ou couplés). Malheureusement, il ne s'agit pas d'un commutateur parfait et peut causer un couplage résiduel dans le mode où les qubits devraient être découplés.

Pour le qubit à terminaux multiples de la Figure $3.10 \mathrm{~b}$ ), le couplage qubit-qubit est possible grâce à un $2 \mathrm{DEG}$ supplémentaire reliant les qubits. Un voltage de grille sur le 2DEG permet de connecter ou déconnecter une paire de qubits $[162,177]$.

Finalement, Blatter et al. ont suggéré un mécanisme basé sur les jonctions $\pi / 2$ pour coupler et découpler des qubits [161]. Dans cette approche toutefois, chaque commutateur requiert un grand nombre d'éléments ( 6 jonctions ' 0 ', une jonction $\pi$ et une jonction $\pi / 2$ par commutateur) et impose des contraintes de fabrication importante (certaines paires de jonctions doivent avoir la même énergie Josephson). Il semble donc, encore une fois de plus, que cette approche ne soit pas utile en pratique. 


\subsubsection{Designs récents}

Pour les qubits phase-charge de Saclay et le design basé sur une seule jonction Josephson (CBJJ) exposés à la section §3.4.3, peu de suggestions de couplage ont jusqu'à présent été présentées.

Pour le qubit phase-charge, un couplage capacitif semble être une solution naturelle. Comme décrit précédemment, un tel couplage donne une interaction ayant la forme $\sigma_{z} \sigma_{z}$ dans la base de charge. Toutefois, puisque ce qubit opère dans une base formée de superpositions symétrique et antisymétrique d'états de charge (i.e., $(|n=0\rangle \pm|n=1\rangle) / \sqrt{2}$ dans l'approximation à deux charges valide pour $E_{J} / E_{C}$ petit $)$, le remplacement (3.23) n'est plus valide. Dans cette nouvelle base logique, l'opérateur de charge prend plutôt la forme $\hat{n} \rightarrow\left(\mathbb{I}-\beta \sigma_{x}\right) / 2$. Le facteur $\beta$ peut facilement être évalué par diagonalisation exacte. Au point d'opération du qubit phase-charge, celuici prend la valeur $\beta=1$ lorsque l'énergie de charge domine par rapport à l'énergie Josephson et croît avec une augmentation du rapport $E_{J} / E_{C}$. Dans cette base, l'interaction a donc la symétrie $\sigma_{x} \sigma_{x}$. Ce type d'interaction capacitive est probablement le plus simple à réaliser en pratique mais a les désavantages énoncés plus haut.

Pour le qubit du type CBJJ, un couplage capacitif a été suggéré par J. Martinis [178] mais n'a pas été analysé en détail. Dans la section suivante, cette idée est explorée de façon plus approfondie. On appliquera ensuite les résultats de cette étude au couplage ajustable d'une paire de qubits phase-charge. Cette approche originale peut toutefois s'appliquer à d'autres types de qubits. Les deux prochaines sections sont basées sur la référence [179].

\subsection{Interaction résonante entre CBJJ}

La figure 4.2 présente deux qubits du type CBJJ couplés par une capacité. En utilisant l'approche décrite au chapitre 3 , on obtient facilement l'hamiltonien de ce système :

$$
\mathcal{H}=\left(\frac{p_{1}^{2}}{2 \tilde{C}_{j 1}}-E_{j 1} \cos \phi_{1}-\frac{\Phi_{0}}{2 \pi} I_{1} \phi_{1}\right)+\left(\frac{p_{2}^{2}}{2 \tilde{C}_{j 2}}-E_{j 2} \cos \phi_{2}-\frac{\Phi_{0}}{2 \pi} I_{2} \phi_{2}\right)+\frac{p_{1} p_{2}}{\tilde{C}_{c}}
$$




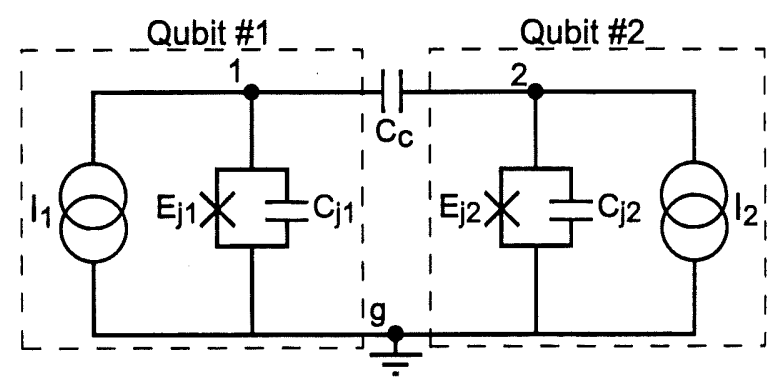

FIG. 4.2: Paire de qubits CBJJ couplés par une capacité $C_{c}$.

Ici, $p_{i}$ est la charge au point $i$ et $\phi_{i}$ la différence de phase de la jonction $i$. Les capacités effectives sont

$$
\begin{aligned}
& \tilde{C}_{j 1}=C_{j 1}+\left(\frac{1}{C_{j 2}}+\frac{1}{C_{c}}\right)^{-1}, \\
& \tilde{C}_{j 2}=C_{j 2}+\left(\frac{1}{C_{j 1}}+\frac{1}{C_{c}}\right)^{-1}, \\
& \tilde{C}_{c}=C_{j 1} C_{j 2}\left(\frac{1}{C_{j 1}}+\frac{1}{C_{j 2}}+\frac{1}{C_{c}}\right) .
\end{aligned}
$$

Les termes entre les première et seconde paires de parenthèses dans (4.3) décrivent respectivement le premier et second qubits. Le dernier terme décrit l'interaction entre ceux-ci. Puisqu'il s'agit d'un couplage capacitif, on retrouve une interaction du type charge-charge. Pour les qubits CBJJ la charge n'est pas un bon nombre quantique et on ne peut faire le remplacement (3.23) vers les matrices de Pauli.

De façon à faciliter l'analyse, on prend ici $C_{j 1}=C_{j 2} \equiv C_{j}$ et $E_{j 1}=E_{j 2} \equiv E_{j}$. Le cas plus réaliste où les jonctions ne sont pas identiques ne pose pas de problème en pratique. Comme il deviendra apparent plus loin, il suffit alors de régler les courants appliqués en conséquence. De plus, on choisit de travailler avec le même type de jonctions et dans le même régime que l'expérience de la référence [7]. Tel que décrit à la section $\S 3.4 .3$ et illustré à la figure 3.12 , on ajuste donc le courant dans chacune - des jonctions de sorte qu'il n'y ait que trois niveaux par puits. Les courants appliqués s'approchent donc du courant critique des jonctions. Dans ce cas, l'énergie potentielle d'un puits s'approxime bien par un potentiel cubique $U(\tilde{\phi})=\frac{1}{2} m \omega_{p}^{2} \tilde{\phi}^{2}(1-\tilde{\phi} / \Delta \tilde{\phi})[129]$. Les paramètres importants de ce potentiel sont présentés à la figure 4.3. À l'aide de cette approximation, on peut donc traiter les jonctions comme si elles étaient des 


\subsection{INTERACTION RÉSONANTE ENTRE CBJJ}

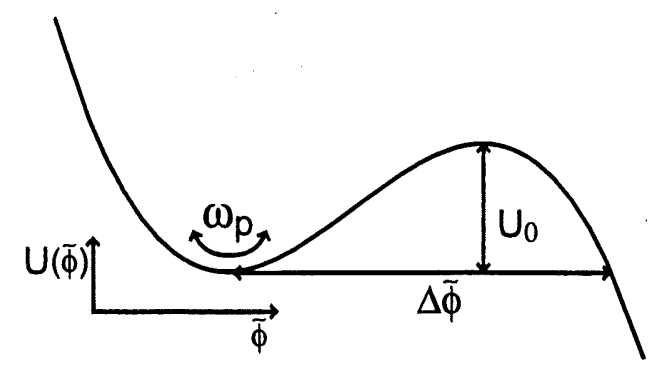

FIG. 4.3: Potentiel cubique associé à une jonction Josephson à travers laquelle est appliqué un courant s'approchant du courant critique. La coordonnée dans le puits est $\tilde{\phi}=\phi-\phi_{0}$, où $\phi$ est la différence de phase de la jonction et $\phi_{0}$ la position du minimum du potentiel. En bonne approximation, on a $\Delta \tilde{\phi}=\sqrt{18\left(1-I_{b} / I_{c}\right)}$ et $U_{0}=2^{3 / 2} \Phi_{0} I_{c}\left(1-I_{b} / I_{c}\right)^{3 / 2} / 3 \pi$. La fréquence plasma $\omega_{p}$ est la fréquence des petites oscillations dans le puits $\omega_{p}=\sqrt{m^{-1}\left(\partial^{2} U / \partial \tilde{\phi}^{2}\right)_{\tilde{\phi}=0}}$ où la 'masse' est donnée par $m=C\left(\Phi_{0} / 2 \pi\right)^{2}$ avec $C$ la capacité totale de la jonction. Figure adaptée de la référence [129].

oscillateurs anharmoniques.

Avec cette analogie, le terme d'interaction dans l'hamiltonien (4.3) devient un couplage impulsion-impulsion entre les oscillateurs. Afin de déterminer la façon dont ce couplage agit sur les états logiques, on commence par trouver les états propres du système couplé. Pour arriver à ce résultat, on exprime d'abord l'hamiltonien (4.3) dans la base des états de chacun des qubits $\{|0\rangle,|1\rangle,|2\rangle\} \otimes\{|0\rangle,|1\rangle,|2\rangle\}=\{|00\rangle,|01\rangle$, $|02\rangle,|10\rangle,|11\rangle,|12\rangle, \ldots\}$, où l'index de gauche est pour le qubit \#1 et celui de droite pour le qubit de \#2. Pour un oscillateur anharmonique cela ne peut évidemment pas être fait exactement. De plus, puisque le courant appliqué s'approche du courant critique, l'anharmonicité n'est pas faible et le premier ordre de la théorie de perturbation ne sera typiquement pas suffisant. Le calcul est donc réalisé numériquement en exprimant d'abord les éléments de matrice de l'hamiltonien de l'oscillateur anharmonique dans la base des états propres de l'oscillateur harmonique. On diagonalise le résultat obtenu en utilisant environ 20 états de base de l'oscillateur harmonique. Pour le terme d'interaction, on exprime les éléments de matrice de l'impulsion dans la base $\{|0\rangle,|1\rangle,|2\rangle\}$. Ces éléments de matrice sont calculés en utilisant les états propres obtenus de la diagonalisation numérique. Dans la base du nombre d'occupation de l'oscillateur harmonique, la mécanique quantique élémentaire nous donne pour les 
impulsions entrant dans le terme d'interaction

$$
p_{i} \mapsto i \frac{2 \pi}{\Phi_{0}} \sqrt{\frac{m \hbar \omega_{p i}}{2}}\left(a_{i}^{\dagger}-a_{i}\right)
$$

où la 'masse' $m$ et la fréquence plasma $\omega_{p i}$ de la jonction $i$ sont données respectivement par $m=\tilde{C}_{j}\left(\Phi_{0} / 2 \pi\right)^{2}$ et $\omega_{p i}=\sqrt{2 \pi I_{c} / \tilde{C}_{j} \Phi_{0}}\left(1-\left(I_{i} / I_{c}\right)^{2}\right)^{1 / 4}[117,129]$.

Dans le but de se former une intuition pour les résultats numériques, étudions d'abord les résultats de la théorie de perturbation au premier ordre. Dans ce cas, on obtient pour l'impulsion (dans la base $\{|0\rangle,|1\rangle,|2\rangle\}$ )

$$
p_{i}=\frac{2 \pi}{\Phi_{0}} \sqrt{m_{i} \hbar \omega_{p i}}\left(\begin{array}{ccc}
0 & -i / \sqrt{2} & -i \sqrt{2} \sigma_{i} \\
i / \sqrt{2} & 0 & -i \\
i \sqrt{2} \sigma_{i} & i & 0
\end{array}\right),
$$

où $\sigma_{i}=-\sqrt{\hbar^{3} \omega_{p i} I_{c} / 2 m\left(I_{i}-I_{c}\right)} / 6$ est le facteur d'anharmonicité : $H_{\text {anharmonique }}=$ $\hbar \omega(N+1 / 2)+\sigma \hbar \omega X^{3}[180]$. Prenant le produit tensoriel de $p_{1}$ et $p_{2}$, en ajoutant ce résultat à l'hamiltonien des deux jonctions découplées, on obtient

$H=$

$$
\left(\begin{array}{ccccccccc}
E_{1} & 0 & 0 & 0 & -\gamma / 2 & -\gamma \sigma & 0 & -\gamma \sigma & -2 \gamma \sigma^{2} \\
0 & E_{2} & 0 & \gamma / 2 & 0 & -\gamma / \sqrt{2} & \gamma \sigma & 0 & -\sqrt{2} \gamma \sigma \\
0 & 0 & E_{3} & \gamma \sigma & \gamma \sqrt{2} & 0 & 2 \gamma \sigma^{2} & \sqrt{2} \gamma \sigma & 0 \\
0 & \gamma / 2 & \gamma \sigma & E_{4} & 0 & 0 & 0 & -\gamma / \sqrt{2} & -\sqrt{2} \gamma \sigma \\
-\gamma / 2 & 0 & \gamma / \sqrt{2} & 0 & E_{5} & 0 & \gamma / \sqrt{2} & 0 & -\gamma \\
-\gamma \sigma & -\gamma / \sqrt{2} & 0 & 0 & 0 & E_{6} & \sqrt{2} \gamma \sigma & \gamma & 0 \\
0 & \gamma \sigma & 2 \gamma \sigma^{2} & 0 & \gamma / \sqrt{2} & \sqrt{2} \gamma \sigma & E_{7} & 0 & 0 \\
-\gamma \sigma & 0 & \sqrt{2} \gamma \sigma & -\gamma / \sqrt{2} & 0 & \gamma & 0 & E_{8} & 0 \\
-2 \gamma \sigma^{2} & -\sqrt{2} \gamma \sigma & 0 & -\sqrt{2} \gamma \sigma & -\gamma & 0 & 0 & 0 & E_{9}
\end{array}\right)
$$

où $E_{1}=E_{10}+E_{20}, E_{2}=E_{10}+E_{21}, E_{3}=E_{10}+E_{22}, \ldots, E_{9}=E_{12}+E_{22}$. Ici, $E_{i j}$ est l'énergie du $j^{\text {ième }}$ niveau du qubit $i$ et $\gamma \equiv \hbar \sqrt{\omega_{p 1} \omega_{p 2}} \tilde{C}_{j} / \tilde{C}_{c}$.

Attardons-nous tout d'abord au sous-espace décrit par les états $\{|01\rangle,|10\rangle\}$. Dans ce sous-espace, l'hamiltonien prend la forme

$$
H_{2}=\left(\begin{array}{cc}
E_{2} & \gamma / 2 \\
\gamma / 2 & E_{4}
\end{array}\right)
$$




\subsection{INTERACTION RÉSONANTE ENTRE CBJJ}

Avec $E_{2}=E_{4}$, c'est-à-dire en ajustant les courants de sorte que $E_{11}-E_{10}=E_{21}-E_{20}$, alors sans le couplage $\gamma$ les états $|01\rangle$ et $|10\rangle$ sont dégénérés. L'interaction lève cette dégénérescence et les nouveaux états propres sont des superpositions de $|10\rangle$ et $|01\rangle$ : $(|10\rangle \pm|01\rangle) / \sqrt{2}$. Il s'agit d'états d'enchevêtrement maximal des deux qubits. Ainsi, si le système est initialement préparé dans l'état $|10\rangle$ alors, sous l'action de ce couplage, il oscillera de façon cohérente entre $|10\rangle$ et $|01\rangle$ par échange d'un 'quanta' entre les jonctions.

Ceci n'est malheureusement pas une description complète du système. Comme on le voit clairement de (4.7), les vrais états propres du système couplé (sans tenir compte de l'environnement pour l'instant) feront intervenir d'autres états. En particulier, ceux-ci feront intervenir l'état $|2\rangle$. Tel que décrit à la section \$3.4.3, cet état se trouve près du haut de la barrière de potentiel et son taux tunnel vers le continuum est grand. Le temps de vie de ce niveau est très court et la présence de $|2\rangle$ dans les états propres aura pour effet de réduire le temps de cohérence du système.

Afin d'évaluer jusqu'à quel point cette fuite hors du sous-espace $\{|01\rangle,|10\rangle\}$ est importante, on a maintenant recours au calcul numérique. Les étapes de calcul sont les mêmes qu'énoncées précédemment, à la différence que l'hamiltonien (4.7) est maintenant obtenue à partir des résultats de la diagonalisation numérique. Dans ce calcul, on choisit les valeurs des paramètres citées à la référence [7] : $C_{j}=6 \mathrm{pF}$ et $I_{c}=21 \mu \mathrm{A}$. De plus, on prend pour le courant appliqué $I=20.8 \mu \mathrm{A}$ de sorte qu'il n'y a que trois niveaux dans chaque puits. La capacité de couplage est $C_{c}=25 \mathrm{fF}$. Cette valeur est choisie de façon à minimiser la participation de l'état $|2\rangle$ tout en gardant un temps caractéristique raisonnable pour l'interaction qubit-qubit (voir plus loin).

De cette façon, on obtient pour les états propres faisant intervenir $|01\rangle$ et $|10\rangle$ fortement :

$$
\begin{aligned}
& \left|\phi_{2}\right\rangle=0.707|01\rangle-0.707|10\rangle+10^{-4}|02\rangle+10^{-3}|12\rangle+10^{-4}|20\rangle+10^{-3}|21\rangle ; \\
& \left|\phi_{3}\right\rangle=0.707|01\rangle+0.707|10\rangle+10^{-4}|02\rangle+10^{-3}|12\rangle+10^{-4}|20\rangle+10^{-3}|21\rangle .
\end{aligned}
$$

Comme on est en droit de s'attendre, les états propres du système s'approchent des états d'enchevêtrement maximal. Toutefois, il existe une faible probabilité, de l'ordre de $10^{-6}$, de trouver le système dans l'état $|2\rangle$. Les autres états propres font aussi intervenir l'état $|2\rangle$ avec une faible probabilité. On observe que la fuite est la plus importante pour l'état propre s'approchant de $|11\rangle$ qui a une probabilité d'occupation 
de l'état $|2\rangle$ de l'ordre de $10^{-4}$. Très grossièrement, le temps de vie d'une superposition d'états ayant pour l'état $|2\rangle$ une probabilité d'occupation $\rho_{22}$ est de l'ordre de $\left(\rho_{22} \Gamma_{2}\right)^{-1}$. Puisque $\Gamma_{2} / \Gamma_{1} \sim 10^{3}$ [7], alors une probabilité d'occupation $\rho_{22}$ plus petite que $\sim 10^{-3}$ ne devrait pas changer de façon dramatique le temps de vie des qubits.

Lorsque les qubits ne sont pas en résonance, $E_{2} \neq E_{4}$, leur dynamique est découplée. Afin de vérifier que cela est bien le cas, on garde le courant dans le premier qubit à $20.8 \mu \mathrm{A}$ et on l'ajuste pour le second à $20.43 \mu \mathrm{A}$. Avec cette valeur de courant, 11 niveaux doivent être pris en considération pour le second qubit. Dans ce cas, les états propres (4.9) deviennent

$$
\begin{aligned}
& \left|\phi_{2}\right\rangle=-0.007|01\rangle+0.999|10\rangle \\
& \left|\phi_{3}\right\rangle=0.999|01\rangle+0.007|10\rangle
\end{aligned}
$$

où la participation d'autres états avec une probabilité de l'ordre de $10^{-6}$ a été omise. Comme il se doit, les états $|01\rangle$ et $|10\rangle$ sont effectivement découplés. Encore une fois, le calcul des autres états propres montre que c'est pour l'état propre $|11\rangle$ que la participation de l'état $|2\rangle$ est la plus grande. Celui-ci se mélange avec $|20\rangle$ et $|02\rangle$ qui ont le même nombre de 'quanta'. Notons que le mapping vers un potentiel cubique n'est valide que pour des courants s'approchant du courant critique. L'approche utilisée ici ne devrait donc pas être employée pour des courants beaucoup plus faibles.

À l'aide de ces résultats, on s'intéresse maintenant aux opérations logiques quantiques qu'il est possible de réaliser avec cette interaction. On prend ici les qubits en résonance $E_{2}=E_{4}$. Puisque l'on suppose que les qubits ont les mêmes caractéristiques, les paires d'états $\{|02\rangle,|20\rangle\}$ et $\{|12\rangle,|21\rangle\}$ seront aussi en résonance. Ces états ne sont toutefois pas directement impliqués dans la dynamique (qui nous intéresse) et ils seront omis pour l'instant. Ainsi, en omettant des termes d'au plus $O\left(10^{-4}\right)$ en probabilité, les états propres pertinents sont :

$$
\begin{aligned}
& \left|\phi_{1}\right\rangle=|00\rangle \\
& \left|\phi_{2}\right\rangle=(|10\rangle-|01\rangle) / \sqrt{2} ; \\
& \left|\phi_{3}\right\rangle=(|10\rangle+|01\rangle) / \sqrt{2} ; \\
& \left|\phi_{4}\right\rangle=|11\rangle .
\end{aligned}
$$

À ces états correspondent les énergies propres $E_{1}^{\prime}$ à $E_{4}^{\prime}$. 


\subsection{INTERACTION RÉSONANTE ENTRE CBJJ}

Imaginons que le premier qubit est préparé au temps $t=0$ dans un état arbitraire $a|0\rangle+b|1\rangle$ et que le second est dans son fondamental $|0\rangle$. En développant l'état du système total au temps $t=0$ dans la base des états propres (4.11), on trouve qu'au temps $t=T$ celui-ci sera dans l'état

$$
|\psi(T)\rangle=a e^{-i E_{1} T / \hbar}|00\rangle+b e^{-i \tilde{E} T / \hbar}\left[\cos \left(\frac{\gamma T}{2 \hbar}\right)|01\rangle-i \sin \left(\frac{\gamma T}{2 \hbar}\right)|10\rangle\right] .
$$

Pour obtenir cette expression, on a utilisé le fait que $E_{2}^{\prime}=\tilde{E}-\gamma / 2$ et $E_{3}^{\prime}=\tilde{E}+\gamma / 2$, où $\tilde{E}=E_{q 0}+E_{b 1}$. Il apparaît de (4.12) que l'hamiltonien du système couplé agit comme $e^{-i \tilde{E} t / \hbar} e^{-i \sigma_{x} \gamma T / 2 \hbar}$ sur le sous-espace $\{|01\rangle,|10\rangle\}$ et comme un facteur de phase (relatif) sur les autres états.

Tel que mentionné précédemment, l'effet de cette interaction peut être observé expérimentalement en préparant d'abord les qubits, alors qu'ils sont hors résonance, dans l'état $|10\rangle$. On les place ensuite en résonance pendant un temps $T$ pour finalement mesurer l'état d'un des deux qubits. En répétant la procédure pour différentes valeurs de $T$, la probabilité de mesurer le qubit dans l'état $|1\rangle$ oscillera à la fréquence $\gamma / h$. Avec les valeurs des différents paramètres données précédemment, la période correspondant à ces oscillations est d'environ 40ns, ce qui est raisonnable pour le qubit CBJJ [7]. Pour des qubits découplés, de telles oscillations surviendront seulement sous application d'un courant alternatif. L'observation de cette oscillation de probabilité pour deux qubits couplés et sans application de courant alternatif constitue donc une démonstration indirecte de l'enchevêtrement entre les qubits.

Dans le contexte du calcul quantique, cette évolution sur une demi-période $h / 2 \gamma$ correspond à l'opération logique SWAP dont on a déjà discuté à la section §1.7. Si on laisse le système évoluer plutôt sur un quart de période, on réalise alors l'opération 'racine de swap' (à des facteurs de phase relative près)

$$
\sqrt{\mathrm{SWAP}}=\left(\begin{array}{cccc}
1 & & & \\
& 1 / \sqrt{2} & -1 / \sqrt{2} & \\
& 1 / \sqrt{2} & 1 / \sqrt{2} & \\
& & & 1
\end{array}\right) .
$$

Cette opération est équivalente au $C_{\text {NOT }}$ et permet le calcul quantique universel sur les qubits CBJJ. 
Notons finalement qu'il est possible de réduire le mélange à l'état $|2\rangle$ en optimisant la valeur des paramètres. Par exemple, en réduisant la valeur de la capacité de couplage $C_{c}$, la participation de $|2\rangle$ diminue. Une telle diminution de $C_{c}$ implique toutefois une diminution de la fréquence $\gamma / h$, ce qui n'est pas pratique du point de vue $\mathrm{du}$ calcul quantique. Mentionnons finalement que le couplage entre les qubits doit être ajusté adiabatiquement par rapport à l'énergie entre les niveaux des qubits ( $1 n s)$ mais plus rapidement que la fréquence d'oscillations $\gamma / h$. Ceci laisse une fenêtre de temps raisonnable.

\subsubsection{Déphasage et relaxation du circuit résonant}

En plus de la fuite hors des états logiques des jonctions, il est important de considérer le déphasage et la relaxation. L'effet des fluctuations de courant sur une CBJJ a été étudié par Martinis et al. [181]. On ne considérera ici que l'effet des fluctuations sur le système couplé près de la résonance. On ne s'intéressera donc qu'au sous-espace $\{|01\rangle,|10\rangle\}$ avec comme hamiltonien (4.8) obtenu au premier ordre en anharmonicité.

En omettant un facteur proportionnel à l'identité, on commence par réécrire cet hamiltonien sous la forme

$$
\begin{aligned}
H_{2} & =\frac{1}{2}\left(\begin{array}{cc}
\delta E & \gamma \\
\gamma & -\delta E
\end{array}\right) \\
& =\frac{\delta E}{2} \sigma_{z}+\frac{\gamma}{2} \sigma_{x},
\end{aligned}
$$

où $\delta E=E_{2}-E_{4}$. Les états propres sont [108] :

$$
\begin{aligned}
& |\uparrow\rangle=\cos \frac{\theta}{2}|0\rangle+\sin \frac{\theta}{2}|1\rangle \\
& |\downarrow\rangle=-\sin \frac{\theta}{2}|0\rangle+\cos \frac{\theta}{2}|1\rangle
\end{aligned}
$$

avec $\theta=\tan ^{-1}(\gamma / \delta E)$. À la résonance, $\delta E=0$ et $\theta=\pi / 2$. Dans cette base, on peut écrire

$$
H_{2}=\frac{\Delta E}{2} \tau_{z}
$$




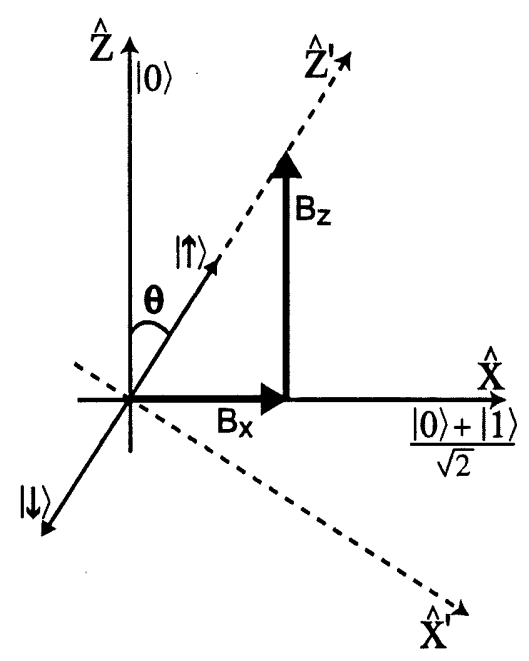

FIG. 4.4: États logiques $\{|0\rangle,|1\rangle\}$ et états propres $\{|\uparrow\rangle,|\downarrow\rangle\}$ de l'hamiltonien à un qubit $B_{z} / 2 \sigma_{z}+B_{x} / 2 \sigma_{x}$ sur la sphère de Bloch. Dans le cas qui nous intéresse ici, $B_{z}=\delta E$ et $B_{x}=\gamma$. Les états logiques sont alignés selon $\pm \hat{z}$ et les états propres selon $\pm \hat{z}^{\prime}$ Figure adaptée de la référence [134].

où $\Delta E=\sqrt{\delta E^{2}+\gamma^{2}}$ et les $\tau_{i}$ sont les matrices de Pauli dans la base des états propres de $H_{2}$ :

$$
\begin{aligned}
\sigma_{z} & =\cos \theta \tau_{z}-\sin \theta \tau_{x} \\
\sigma_{x} & =\sin \theta \tau_{z}+\cos \theta \tau_{x} .
\end{aligned}
$$

Une représentation sur la sphère de Bloch de ces différentes bases se trouve à la figure 4.4.

Dans ce système, des sources importantes de décohérence sont les fluctuations de courant $\delta I_{b 1}(t)$ et $\delta I_{b 2}(t)$ sur chacune des jonctions. Les variations de la fréquence plasma de la jonction $i$ dues aux fluctuations de courant de la source $I_{b i}$ sont données par

$$
\delta \omega_{p i}(t)=-\frac{1}{2} \omega_{p i} \frac{I_{b i} \delta I_{b i}(t)}{I_{c}^{2}-I_{b i}^{2}} .
$$

Ces fluctuations affectent les niveaux d'énergie des jonctions et le couplage $\gamma$. En se limitant au premier ordre en $\delta I$ pour $\delta E$ et $\gamma$ et au premier ordre en anharmonicité pour $\delta E$, on obtient l'hamiltonien système-bain suivant :

$$
H_{S B}=\frac{\hbar}{2}\left(-\delta \omega_{p 1}+\delta \omega_{p 2}\right) \sigma_{z}+\frac{\gamma}{4}\left(\frac{\delta \omega_{p 1}}{\omega_{p 1}}+\frac{\delta \omega_{p 2}}{\omega_{p 2}}\right) \sigma_{x}
$$


En utilisant le changement de base (4.17), on a finalement, dans la base des états propres de $\mathrm{H}_{2}$,

$$
\begin{aligned}
H_{S B}= & \frac{\tau_{z}}{2}\left(\delta \omega_{p 1}\left[\frac{\gamma}{2} \frac{\sin \theta}{\omega_{p 1}}-\hbar \cos \theta\right]+\delta \omega_{p 2}\left[\frac{\gamma}{2} \frac{\sin \theta}{\omega_{p 2}}+\hbar \cos \theta\right]\right) \\
& +\frac{\tau_{x}}{2}\left(\delta \omega_{p 1}\left[\frac{\gamma}{2} \frac{\cos \theta}{\omega_{p 1}}+\hbar \sin \theta\right]+\delta \omega_{p 2}\left[\frac{\gamma}{2} \frac{\cos \theta}{\omega_{p 2}}-\hbar \sin \theta\right]\right) \\
\equiv & \tau_{z}\left(X_{z 1}+X_{z 2}\right)+\tau_{x}\left(X_{x 1}+X_{x 2}\right),
\end{aligned}
$$

où les $X_{\alpha i}$ sont les opérateurs de bain. Puisqu'il y a deux sources de courant, le système est couplé à deux environnements indépendants. Les termes en $\tau_{z}$ sont responsables du déphasage. Les termes en $\tau_{x}$ permettent les transitions entre les états propres de $\mathrm{H}_{2}$ et causent ainsi la relaxation.

Afin de déterminer les taux de déphasage et de relaxation, on a d'abord pour les fréquences plasma

$$
\left\langle\delta \omega_{p i} \delta \omega_{p i}\right\rangle_{\omega}=\frac{\omega_{p i}^{2}}{4} \frac{I_{b i}^{2}}{\left(I_{c}^{2}-I_{b i}^{2}\right)^{2}}\langle\delta I \delta I\rangle_{\omega} .
$$

À l'équilibre thermodynamique, le théorème fluctuation-disspation donne pour la fonction de corrélation courant-courant

$$
\langle\delta I \delta I\rangle_{\omega}=\frac{\hbar \omega}{R_{I}} \operatorname{coth}\left(\frac{\hbar \omega}{2 k_{B} T}\right) .
$$

On suppose ici pour simplifier la discussion que l'impédance de chacune des jonctions est telle que $\operatorname{Re}[Z(\omega)]=R_{I}$.

Comme on le voit de (4.20) les fonctions de corrélation des opérateurs de bain

$$
\left\langle X_{\alpha i} X_{\alpha i}\right\rangle_{\omega}=\hbar J_{\alpha i}(\omega) \operatorname{coth}\left(\frac{\hbar \omega}{2 k_{B} T}\right)
$$

font intervenir $\left\langle\delta \omega_{p i} \delta \omega_{p i}\right\rangle_{\omega}$ et par conséquent $\langle\delta I \delta I\rangle_{\omega}$. En portant (4.22) dans (4.21) puis en tirant les préfacteurs appropriés de (4.20), on obtient les densités spectrales suivantes :

$$
\begin{aligned}
& J_{z 1}(\omega)=\left\{\frac{\omega_{p 1}}{4} \frac{I_{b 1}}{I_{c}^{2}-I_{b 1}^{2}}\left[\frac{\gamma}{2} \frac{\sin \theta}{\omega_{p 1}}-\hbar \cos \theta\right]\right\}^{2} \frac{\omega}{R_{I}} \\
& J_{z 2}(\omega)=\left\{\frac{\omega_{p 2}}{4} \frac{I_{b 2}}{I_{c}^{2}-I_{b 2}^{2}}\left[\frac{\gamma}{2} \frac{\sin \theta}{\omega_{p 2}}+\hbar \cos \theta\right]\right\}^{2} \frac{\omega}{R_{I}}
\end{aligned}
$$




$$
\begin{aligned}
& J_{x 1}(\omega)=\left\{\frac{\omega_{p 1}}{4} \frac{I_{b 1}}{I_{c}^{2}-I_{b 1}^{2}}\left[\frac{\gamma}{2} \frac{\cos \theta}{\omega_{p 1}}+\hbar \sin \theta\right]\right\}^{2} \frac{\omega}{R_{I}} \\
& J_{x 2}(\omega)=\left\{\frac{\omega_{p 2}}{4} \frac{I_{b 2}}{I_{c}^{2}-I_{b 2}^{2}}\left[\frac{\gamma}{2} \frac{\cos \theta}{\omega_{p 2}}-\hbar \sin \theta\right]\right\}^{2} \frac{\omega}{R_{I}} .
\end{aligned}
$$

De la même façon qu'à la section $\S 3.3$, on obtient finalement

$$
\begin{aligned}
& \Gamma_{r}=\frac{1}{2 \hbar}\left(J_{x 1}\left(\omega_{0}\right)+J_{x 2}\left(\omega_{0}\right)\right) \operatorname{coth}\left(\frac{\hbar \omega_{0}}{2 k_{B} T}\right), \\
& \Gamma_{\phi}=\frac{\Gamma_{r}}{2}+2 \pi \alpha \frac{k_{B} T}{\hbar}
\end{aligned}
$$

avec $\alpha=\left(J_{z 1}(\omega)+J_{z 2}(\omega)\right) / 2 \pi \hbar \omega$ à $\omega \rightarrow 0$. Les contributions des différents bains s'additionnent dans leur participation aux taux de déphasage et de relaxation [108].

Pour le qubit CBJJ, un long temps de cohérence ne peut être atteint que si l'environnement de la jonction a une grande impédance. Aux fréquences micro-ondes, celle-ci est toutefois typiquement de l'ordre de $100 \Omega$ [118]. Dans l'expérience décrite à la référence [7], l'environnement de la jonction a été ajusté de sorte que $\operatorname{Re}[Z(\omega)] \approx$ $560 \mathrm{k} \Omega$. Avec cette valeur, exactement à la résonance, on trouve que $\Gamma_{r}^{-1}$ et $\Gamma_{\phi}^{-1}$ sont de l'ordre de la milliseconde. Hors résonance, $\Gamma_{r}^{-1}$ augmente alors que $\Gamma_{\phi}^{-1}$ reste du même ordre de grandeur. C'est le résultat auquel on doit s'attendre puisqu'à la résonance les états propres sont dans la direction $\hat{x}$ sur la sphère de Bloch. C'est donc à ce point que le 'mélange' des états logiques est le plus important. On vérifie aussi de l'hamiltonien système-bain (4.20) qu' à la résonance, le bruit selon $\hat{z}^{\prime}$ est plus faible d'un facteur $\hbar \tilde{C}_{j} / \tilde{C}_{c}$ par rapport au bruit selon $\hat{x}^{\prime}$ et vice-versa loin de la résonance.

Les plus petites valeurs de $\Gamma_{r}^{-1}$ et $\Gamma_{\phi}^{-1}$ obtenues ici sont plus grandes que les temps correspondants pour les jonctions découplées $[7,8]$. Coupler capacitivement une paire de jonctions ne devrait donc pas affecter sérieusement les temps de cohérence du système. Rappelons que l'on ne tient pas compte ici de toutes les sources de bruits et de décohérence présentes dans le système. On ne s'intéresse qu'au bruit dû au couplage des jonctions et non aux sources de décohérence "intrinsèques". En particulier, on n'a pas tenu compte de la présence du continuum. 


\subsection{Interaction résonante pour qubits supraconducteurs}

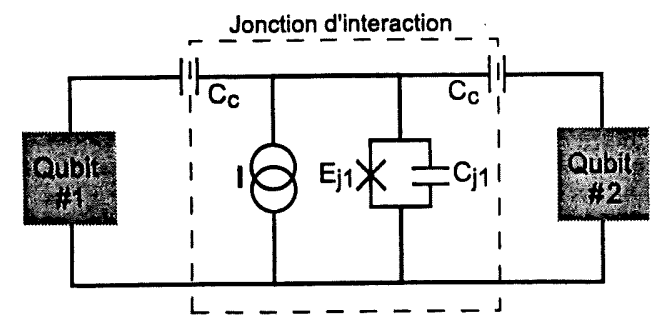

(a)

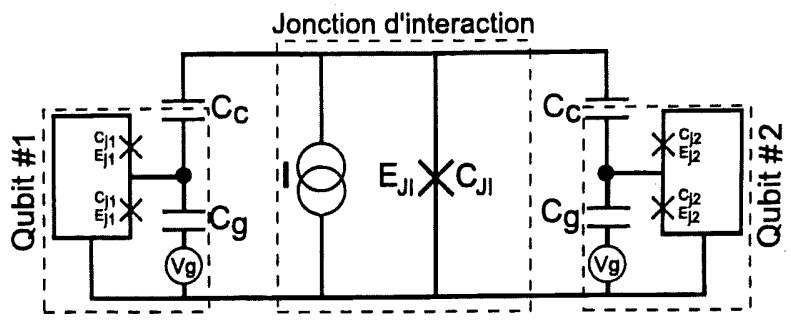

(b)

FIG. 4.5: a) Une paire de qubits supraconducteurs couplés capacitivement à une CBJJ. b) Qubits phase-charge couplés à une jonction Josephson. Celle-ci agit comme système intermédiaire dans l'interaction qubit-qubit.

Le mode d'opération étudié à la section précédente suggère une technique très générale pour faire interagir les qubits supraconducteurs. Le concept est présenté à la figure 4.5 a). Deux bits quantiques sont couplés capacitivement à une CBJJ qui agit comme intermédiaire dans l'interaction. On ajuste le courant dans la jonction intermédiaire de façon à ce qu'elle soit séquentiellement en résonance avec les qubits. Ceux-ci interagissent donc indirectement. Ce mode d'opération est semblable aux cavités électrodynamiques [182] et aux designs d'ordinateurs quantiques basés sur les pièges à ions linéaires [159]. Dans le premier cas, les qubits jouent le rôle des atomes et la jonction celui de la cavité. Ajuster en résonance la jonction avec le qubit correspond à faire passer l'atome dans la cavité. Ḍans la deuxième analogie, la jonction tient le rôle du mode collectif de déplacement des ions dans le piège linéaire.

Un aspect particulièrement intéressant de cette technique est que, hors résonance, la jonction est découplée des qubits. Il est donc possible de contrôler l'interaction qubit-qubit. De plus, l'interaction ne requiert que des manipulations sur la jonction. Il n'y a pas de manipulation directement sur les qubits ce qui peut aider à réduire la décohérence. Mentionnons aussi que les qubits \#1 et \#2 de la figure 4.5 n'ont pas à être du même type, en autant que chacun puisse être connecté capacitivement à la jonction. 
Considérons pour commencer le cas où une paire de qubits phase-charge est connectée à la jonction, figure $4.5 \mathrm{~b}$ ). On considéra à nouveau plus de deux niveaux pour la jonction mais, seulement deux niveaux pour les qubits phase-charge. Afin de coupler un seul qubit à la fois au CBJJ, on choisit les qubits de façon à ce que $\Delta E_{1} \neq \Delta E_{2}$, où $\Delta E_{i}$ est la séparation en énergie entre les états logiques du qubit $i$. Au point d'opération $\left(n_{g}=1 / 2, \Phi=0\right)$, cette séparation est donnée par $2 E_{J i}$. Il est possible d'ajuster cette différence en appliquant, par exemple, un flux dans la boucle du qubit. Cependant, celui-ci est alors plus sensible aux fluctuations de ces paramètres de contrôle. Cette possibilité ne sera donc pas considérée davantage. En pratique, ceci implique que les paires de jonctions formant les qubits doivent avoir des énergies Josephson différentes $\left[E_{J 1} \neq E_{J 2}\right.$ à la Figure $\left.\left.4.5 \mathrm{~b}\right)\right]$. Notons que d'autres façon de moduler $\Delta E$ existent [183].

Comme dans le cas des deux jonctions, le terme d'interaction qubit-jonction couple la charge de la jonction $p_{J}$ à la charge du qubit $p_{q}$. Dans la base de calcul du qubit $i$, on a donc un terme de la forme $\sigma_{x i} p_{J} / \tilde{C}_{c}$. La description du système est essentiellement identique à celle des deux jonctions couplées. Les seules modifications sont pour le coefficient de couplage qui prend ici la forme $\gamma_{s} \equiv 2 e\left(2 \pi / \Phi_{0}\right) \sqrt{m \hbar \omega_{p}} / \tilde{C}_{c}$ et la renormalisation des capacités effectives.

Afin de réaliser une opération à deux qubits, on fait interagir les qubits séquentiellement. À la fin de l'opération, il est important que la jonction ne soit pas enchevêtrée avec les qubits. L'enchevêtrement résiduel affecte leur cohérence et introduit des erreurs dans le calcul. Afin d'éviter ce type d'enchevêtrement résiduel, on suggère la séquence d'opérations suivante. Alors que la jonction d'interaction est hors résonance par rapport aux deux qubits, on prépare les qubits dans un état arbitraire et la jonction dans son fondamental

$$
\begin{gathered}
(a|00\rangle+b|01\rangle+c|10\rangle+d|11\rangle) \otimes|0\rangle \\
=a|000\rangle+b|010\rangle+c|100\rangle+d|110\rangle .
\end{gathered}
$$

Les deux premiers index sont pour les qubits et le troisième pour la jonction. La jonction est d'abord ajustée à la séparation d'énergie $\Delta E_{1}$ du premier qubit pour un temps $t_{1}$ tel que $\gamma_{s 1} t_{1} / 2 \hbar=\pi / 2$. Elle est ensuite réglée à $\Delta E_{2}$ afin d'interagir avec le second qubit pendant un temps $t_{2}$ choisi de façon à ce que $\gamma_{s 2} t_{2} / 2 \hbar=\pi / 4$. 


\subsection{INTERACTION RÉSONANTE POUR QUBITS SUPRACONDUCTEURS}

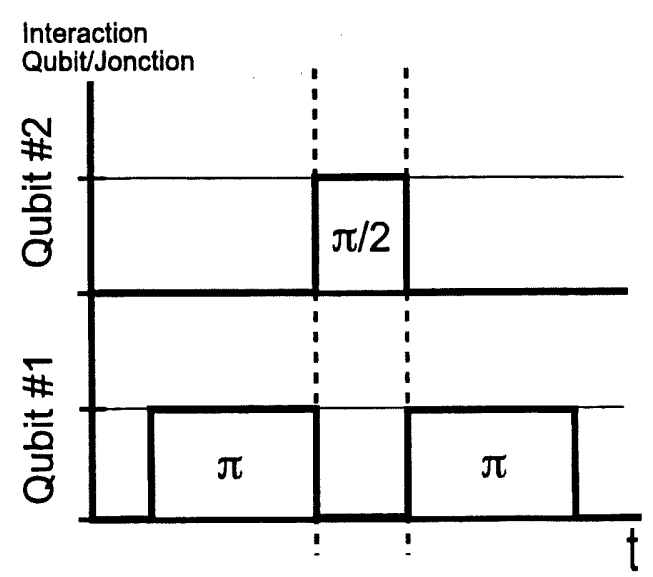

FIG. 4.6: Séquence d'interaction qubits-jonction pour la réalisation de l'opération $\sqrt{\mathrm{SWAP}}$. La première impulsion $\pi$ échange l'état du premier qubit et de la jonction. L'impulsion $\pi / 2$ enchevêtre la jonction et le second qubit. Le rôle de la dernière impulsion est de transférer l'enchevêtrement vers le premier qubit. Ici, 'ouvrir' l'interaction entre le qubit $i$ et la jonction signifie ajuster le courant dans la jonction de façon à ce que la différence entre ses deux premiers niveaux d'énergie soit $\Delta E_{i}$. Lorsque l'interaction n'est pas en action, des impulsions de refocalisation sont appliquées sur les qubits afin de freiner leur évolution (ces impulsions ne sont pas illustrées).

Finalement, elle est à nouveau ajustée à $\Delta E_{1}$ pour une seconde période $t_{1}$. Cette séquence est présentée schématiquement à la figure 4.6. Notons que, comme en RMN, ces qubits ont une différence d'énergie $\Delta E_{i}$ fixe. Une phase relative s'accumule donc toujours entre leurs états logiques (dans le référentiel du laboratoire). On suppose donc ici que le qubit inactif est refocalisé afin d'éliminer cette phase. Suite à ces manipulations, le système sera dans l'état

$$
\begin{aligned}
\left|\psi\left(2 t_{1}+t_{2}\right)\right\rangle=\{ & a e^{-i 2 E_{1}^{\prime 1} t_{1} / \hbar} e^{-i E_{1}^{\prime 2} t_{2} / \hbar}|00\rangle \\
& -\frac{e^{-i \tilde{E}^{2} t_{2} / \hbar}}{\sqrt{2}}\left[b e^{-i\left(E_{1}^{\prime 1}+\tilde{E}^{1}\right) t_{1} / \hbar}+c e^{-2 i \tilde{E}^{1} t_{1} / \hbar}\right]|10\rangle \\
& +\frac{e^{-i \tilde{E}^{2} t_{2} / \hbar}}{\sqrt{2}}\left[b e^{-2 i \tilde{E}^{1} t_{1} / \hbar}-c e^{-i\left(E_{1}^{\prime 1}+\tilde{E}^{1}\right) t_{1} / \hbar}\right]|01\rangle \\
& \left.+d e^{-i 2 \tilde{E}^{1} t_{1} / \hbar} e^{-i E_{4}^{\prime 2} t_{2} / \hbar}|11\rangle\right\} \otimes|0\rangle,
\end{aligned}
$$

où $E_{i}^{j}$ est l'énergie du niveau $i$ du qubit $j$ et les énergies $\tilde{E}^{1,2}$ et $E^{\prime 1,2}$ sont définies 
comme en (4.12).

Comme il se doit, suite à cette séquence, les qubits ne sont pas enchevêtrés avec la jonction. Notons aussi que, sans les facteurs de phase, cette séquence correspond à l'opération $\sqrt{\text { SWAP }}$ (4.13). Cette opération est équivalente au non-contrôlé et, avec les opérations à un qubit, permet le calcul universel. Même en tenant compte des facteurs de phase additionnels, cette séquence d'opérations peut créer des états d'enchevêtrement maximal. Par exemple, si l'on prépare au temps $t=0$ les qubits dans l'état $|01\rangle$ et la jonction dans son fondamental, on obtient au temps $t=2 t_{1}+t_{2}$

$$
\frac{1}{\sqrt{2}}\left(|01\rangle-e^{-i E_{1}^{1} t_{1}}|10\rangle\right) \text {. }
$$

Il s'agit bien d'un état d'enchevêtrement maximal. Puisque la séquence de la figure 4.6 peut créer de tels états, elle est certainement suffisamment versatile pour permettre le calcul quantique universel. En pratique, les facteurs de phase devront être pris en considération lors de la réalisation de portes logiques. Puisque toutes les énergies impliquées dans ces phases sont connues des calculs numériques et qu'elles dépendent de paramètres pouvant être déterminés expérimentalement, ceci devrait être possible.

Comme dans le cas de deux CBJJ couplées, la fuite vers les états d'énergie supérieure de la jonction est possible ici. Afin d'évaluer l'importance de cette fuite, on prend pour la CBJJ les paramètres cités dans la référence [8]: $I_{\mathrm{c}}=147 \mu \mathrm{A}$ et $C_{\mathrm{JI}}=5.8 \mathrm{pF}$. Avec ces valeurs et un courant dans la jonction de $I_{\text {bias }} \gtrsim 0.99 I_{\mathrm{c}}$, la séparation en énergie entre les deux premiers niveaux de la jonction est similaire à la séparation $\Delta E$ pour le qubit phase-charge décrit à la référence [6]. Pour les qubits, on prend donc $C_{\Sigma}=5.5 \mathrm{fF}, \Delta E_{1}$ et $\Delta E_{2}$ correspondant respectivement à la séparation en énergie des premiers niveaux de la jonction pour les courants $I_{\text {bias }}=146.5 \mu \mathrm{A}$ et $146.75 \mu \mathrm{A}$. La capacité de couplage est $C_{\mathrm{c}}=0.1 \mathrm{fF}$. Ces paramètres ont été choisis de façon à minimiser la fuite hors des niveaux logiques de la jonction tout en ayant un temps caractéristique pour l'opération à deux qubits le plus court possible. Avec ces paramètres, ce temps est $h / \gamma_{\mathrm{s}} \sim 85 \mathrm{~ns}$ et est du même ordre de grandeur que la période des oscillations de Rabi observées avec ce qubit [6]. Ces oscillations de Rabi correspondent à l'opération logique à un qubit $\mathrm{R}_{\mathrm{x}}$. Ainsi, le temps caractéristique de l'opération à deux qubits décrite ici est du même ordre de grandeur qu'une rotation à un bit selon $x$. 
Par rapport au cas de la paire de qubits CBJJ couplée, la fuite hors des états logiques est réduite car le qubit phase-charge a (en très bonne approximation) seulement deux niveaux. Puisque le potentiel de la jonction CBJJ est anharmonique, une seule paire de niveaux de la jonction sera en résonance avec le qubit. Toutefois, en présence de deux qubits couplés à la jonction, la densité d'états est plus importante et il est nécessaire de réaliser les opérations logiques plus lentement afin d'éviter les transitions non voulues. Ceci explique le plus grand temps $h / \gamma$ des oscillations cohérentes comparativement au cas de deux CBJJ seulement.

Numériquement, on observe que la participation de l'état $|2\rangle$ de la jonction a une probabilité d'au plus $\sim 10^{-4}$. Diminuer $C_{c}$ aide à réduire cette fuite mais au prix d'un temps $h / \gamma_{\mathrm{s}}$ plus long. Évidemment, $C_{c}$ ne doit pas être trop grande car cela diminuerait l'énergie de charge au-delà des limites utiles. Mentionnons aussi que, comme en (4.9), les états propres du sous-système qubit-jonction ne sont pas exactement les états d'enchevêtrement maximal (4.11). Ce petit écart devra être pris en considération lors de l'application de la séquence de la figure 4.6. Finalement, pour découpler les qubits de la jonction, on ajuste le courant à $I_{\text {bias }}=146.6 \mu \mathrm{A}$. On trouve alors, comme en (4.10), que les états propres du système ne sont pas enchevêtrés (ou le sont faiblement) avec une probabilité d'occupation pour $|2\rangle$ de $\sim 10^{-4}$.

\subsubsection{Interaction qubit-qubit universelle}

La méthode de couplage décrite ici ne fonctionnera qu'avec les qubits pour lesquels le terme d'interaction avec la CBJJ est complètement hors-diagonal dans la base de calcul. Dans la base des deux premiers états de la jonction, la charge $p_{J}$ peut être remplacée par $\sigma_{y}$. Dans cette base réduite, les termes d'interaction doivent donc être de la forme $\sigma_{x q} \sigma_{y J}$ ou $\sigma_{y q} \sigma_{y J}$. Avec ce type d'interaction, les états propres seront comme il se doit des états de Bell. À l'opposé, avec un terme d'interaction de la forme $\sigma_{z q} \sigma_{y J}$, les états propres seront séparables et les oscillations cohérentes par échange d'un quanta n'auront pas lieu. Pour les qubits de charge, le terme d'interaction prendra cette dernière forme.

Afin de rendre cette approche plus versatile, il est intéressant de remarquer que

$$
\mathrm{H} \sigma_{x} \mathrm{H}=\sigma_{z}, \quad \mathrm{H} \sigma_{z} \mathrm{H}=\sigma_{x},
$$




\subsection{INTERACTION RÉSONANTE POUR QUBITS SUPRACONDUCTEURS}

où $\mathrm{H}$ est la transformation d'Hadamard. Si l'on ajuste les paramètres de contrôle de sorte que l'hamiltonien du qubit soit $\Delta E_{q} \sigma_{x} / 2$ et donc l'hamiltonien qubit-jonction

$$
H_{\text {diag }}=\frac{\Delta E_{q}}{2} \sigma_{x q}+\frac{\Delta E_{J}}{2} \sigma_{z J}+\frac{\gamma}{2} \sigma_{z q} p_{J}
$$

alors, lorsque $\mathrm{H}$ n'agit que sur le qubit,

$$
\mathrm{H} H_{\text {diag }} \mathrm{H}=\frac{\Delta E_{q}}{2} \sigma_{z q}+\frac{\Delta E_{J}}{2} \sigma_{z J}+\frac{\gamma}{2} \sigma_{x q} p_{J} \equiv H_{\text {hors-diag. }} .
$$

De même, puisque $H H=\mathbb{I}$, alors $H e^{i H_{\text {diag }} t} H=e^{i H_{\text {hors-diag }} t}$. Ainsi, en appliquant une transformation d'Hadamard sur le qubit avant et après la période d'interaction avec la jonction, le système agira comme s'il avait un hamiltonien d'interaction complètement hors-diagonal.

Grâce à cette astuce simple, il est donc possible d'utiliser la méthode d'interaction proposée à la section précédente avec l'hamiltonien d'interaction $\sigma_{z} \sigma_{y}$. Puisque, avec $\sigma_{x} \sigma_{y}$ et $\sigma_{y} \sigma_{y}$, ce sont les seuls types d'interaction possibles, cette méthode d'interaction est universelle au sens où elle s'applique à tous les qubits pouvant être connectés à une CBJJ.

Notons que ce type d'approche où l'hamiltonien est manipulé à l'aide d'impulsions est standard en RMN. Dans la communauté de l'informatique quantique, ce type d'approche est connu sous le nom de 'recoupling'. Cette approche est présentée en plus de détails à l'annexe $\mathrm{C}$.

\subsubsection{Approximation séculaire}

$\mathrm{Si}$ on ne s'intéresse pas à la fuite hors du sous-espace logique, on peut obtenir les résultats principaux des sections $\S 4.2$ et $\S 4.3$ plus facilement en passant dans le référentiel tournant et en utilisant l'approximation séculaire (rotating-wave approximation) [180]. En effet, tel que décrit à la section précédente, l'hamiltonien décrivant l'interaction entre un qubit et la jonction prend la forme

$$
H=\frac{\Delta E_{q}}{2} \sigma_{z q}+\frac{\Delta E_{J}}{2} \sigma_{z J}+\frac{\gamma}{2} \sigma_{x q} \sigma_{y J}
$$

dans l'approximation à deux niveaux. 


\subsection{INTERACTION RÉSONANTE POUR QUBITS SUPRACONDUCTEURS}

On passe au référentiel tournant pour le qubit et la jonction en utilisant les transformations $(\hbar=1)$

$$
\mathrm{T}_{\mathrm{q}}=e^{i \frac{\Delta E_{q}}{2} \sigma_{z q}} \quad \mathrm{~T}_{\mathrm{J}}=e^{i \frac{\Delta E_{J}}{2} \sigma_{z J}},
$$

de sorte que, dans le référentiel tournant, l'hamiltonien est

$$
\begin{aligned}
\tilde{H} & =\mathrm{T}_{\mathrm{q}} \mathrm{T}_{\jmath} H \mathrm{~T}_{\mathrm{q}}^{\dagger} \mathrm{T}_{\mathrm{J}}^{\dagger}-\frac{\Delta E_{q}}{2} \sigma_{z q}-\frac{\Delta E_{J}}{2} \sigma_{z J} \\
& =i \frac{\gamma}{2}\left(\begin{array}{cc}
-e^{+i\left(\Delta E_{q}+\Delta E_{J}\right) t} \\
e^{-i\left(\Delta E_{q}+\Delta E_{J}\right) t} & -e^{+i\left(\Delta E_{q}-\Delta E_{J}\right) t}
\end{array}\right) .
\end{aligned}
$$

À la résonance, $\Delta E_{q}=\Delta E_{J}$ et

$$
\tilde{H}=i \frac{\gamma}{2}\left(\begin{array}{cccc} 
& & -e^{+2 i \Delta E_{q} t} \\
& & 1 & \\
& & & \\
e^{-2 i \Delta E_{q} t} & & &
\end{array}\right) .
$$

Dans l'approximation séculaire on néglige les termes oscillants rapidement pour finalement obtenir $\tilde{H}=i \gamma\left(\sigma_{+q} \sigma_{-J}-\sigma_{-q} \sigma_{+J}\right) / 2$ que l'on notera $\tilde{H}_{ \pm}$par la suite. L'opérateur d'évolution associé à cet hamiltonien d'interaction a la forme

$$
U_{\gamma}(t)=\left(\begin{array}{cccc}
1 & & & \\
& \cos (\gamma t / 2) & \sin (\gamma t / 2) & \\
& -\sin (\gamma t / 2) & \cos (\gamma t / 2) & \\
& & & 1
\end{array}\right)
$$

Au temps $t=\pi / 2 \gamma$, on obtient bien l'opération $\sqrt{\text { SWAP } a ̀ ~ u n e ~ p h a s e ~ p r e ̀ s ~: ~}$

$$
U_{\gamma}(\pi / 2 \gamma)=\left(\begin{array}{cccc}
1 & & & \\
& 1 / \sqrt{2} & 1 / \sqrt{2} & \\
& -1 / \sqrt{2} & 1 / \sqrt{2} & \\
& & & 1
\end{array}\right) .
$$

Dans le cas de deux CBJJ couplées, on trouve de la même façon que l'hamiltonien d'interaction est plutôt $\tilde{H}_{X Y}=\gamma\left(\sigma_{+q} \sigma_{-J}+\sigma_{-q} \sigma_{+J}\right) / 2=\gamma\left(\sigma_{x q} \sigma_{x J}+\sigma_{y q} \sigma_{y J}\right) / 4$. Il 


\subsection{INTERACTION RÉSONANTE POUR QUBITS SUPRACONDUCTEURS}

s'agit du modèle $X Y$ qui a déjà été étudié à la section $\$ 1.5 .3$ dans le contexte de l'universalité encodée. La réalisation de différentes opérations logiques à l'aide de cet hamiltonien d'interaction a aussi été étudiée en détail à la référence [184].

Lorsque la jonction n'est pas en résonance avec le qubit, on obtient, toujours dans l'approximation séculaire, $\tilde{H}=0$ comme il se doit. La jonction et le qubit n'interagissent alors pas.

\subsubsection{Déphasage et relaxation}

Afin de déterminer les taux de décohérence pour ce système, on peut procéder de la même façon qu'à la section 4.2 .1 et s'intéresser au sous-espace $\{|01\rangle,|10\rangle\}$. On présente ici une approche alternative où l'on s'intéresse à l'effet de l'impédance de la jonction d'interaction sur un qubit.

On ne considère ici qu'un seul qubit couplé à la CBJJ. Le bruit aux bornes de l'impédance $Z(\omega)$ de la jonction est traité comme une source de voltage $V_{f}$ couplé au qubit par la capacité $C_{c}$. Le spectre de fluctuation de cette source est décrit par

$$
\left\langle\left\{V_{f}, V_{f}\right\}\right\rangle_{\omega}=2 \hbar \omega \operatorname{Re}\left[Z_{t}(\omega)\right] \operatorname{coth}\left(\frac{\hbar \omega}{2 k_{B} T}\right),
$$

où $Z_{t}(\omega)$ est l'impédance effective vue entre les bornes de l'impédance $Z(\omega)$ de la jonction :

$$
Z_{t}(\omega)=\frac{Z(\omega)}{1+i \omega C_{e f f} Z(\omega)}
$$

avec

$$
C_{e f f}=C_{J I}+\left(\frac{1}{C_{c}}+\frac{1}{C_{g}+2 C_{J}}\right)^{-1}
$$

Comme à la référence [108], pour obtenir cette impédance effective on a seulement tenu compte de la capacité des jonctions (avec $E_{J}=0$ ).

Dans le cas où un qubit de charge est couplé à la CBJJ, le champ effectif selon $z$ vu par ce qubit devient $B_{z}^{\prime}=4 E_{C}\left(1-2 n_{g}-2 n_{f}\right)=B_{z}-8 E_{C} n_{f}$ avec $n_{f}=C_{c} V_{f} / 2 e$. L'hamiltonien à un qubit s'exprime alors comme

$$
H=-\frac{1}{2} B_{z} \sigma_{z}-\frac{1}{2} B_{x} \sigma_{x}+4 E_{C} n_{f} \sigma_{z}
$$

avec $E_{C}=2 e^{2} / C_{\Sigma}$ et $B_{x}=2 E_{J} \cos \left(\pi \Phi_{x} / \Phi_{0}\right)$, comme en (3.26). 


\subsection{INTERACTION RÉSONANTE POUR QUBITS SUPRACONDUCTEURS}

Comme à la section $\S 4.2 .1$, on passe maintenant à la base des états propres de l'hamiltonien à un qubit

$$
H=-\frac{\Delta E}{2} \tau_{z}+4 E_{C} n_{f}\left(\cos \theta \tau_{z}+\sin \theta \tau_{x}\right),
$$

où $\Delta E=\sqrt{B_{z}^{2}+B_{x}^{2}}$ et $\theta=\tan ^{-1}\left(B_{x} / B_{z}\right)$. De cette dernière expression on tire finalement l'hamiltonien d'interaction système-bain

$$
H_{S B}=e V_{f} \frac{C_{c}}{C_{\Sigma}}\left(\cos \theta \tau_{z}+\sin \theta \tau_{x}\right),
$$

d'où on obtient aisément des relations (3.31) et (3.32) les taux de relaxation et déphasage :

$$
\begin{aligned}
& \Gamma_{r}=\sin ^{2} \theta\left(\frac{e}{\hbar}\right)^{2}\left(\frac{C_{c}}{C_{\Sigma}}\right)^{2}\left[2 \Delta E \operatorname{Re}\left[Z_{t}(\Delta E / \hbar)\right] \operatorname{coth}\left(\frac{\Delta E}{2 k_{B} T}\right)\right], \\
& \Gamma_{\phi}^{*}=\frac{1}{2} \cos ^{2} \theta\left(\frac{e}{\hbar}\right)^{2}\left(\frac{C_{c}}{C_{\Sigma}}\right)^{2}\left[2 \hbar \omega \operatorname{Re}\left[Z_{t}(\omega)\right] \operatorname{coth}\left(\frac{\hbar \omega}{2 k_{B} T}\right)\right]_{\omega \rightarrow 0} .
\end{aligned}
$$

Pour un qubit de charge, on évalue ces taux en utilisant les valeurs de paramètres suivantes : $C_{g}=5 \mathrm{aF}, C_{j}=0.5 \mathrm{fF}, C_{c}=0.1 \mathrm{fF}$ et $C_{J I}=5.8 \mathrm{pF}$. Pour l'impédance de la jonction, on prend $\operatorname{Re}[Z(\omega)] \approx 560 \mathrm{k} \Omega$ tel que vu à la section $\S 3.4$.3. La partie réelle de l'impédance totale (4.40) a la forme d'une lorentzienne centrée à fréquence nulle. Une grande résistance $\operatorname{Re}[Z(\omega)]$ implique donc que presque tout le poids du spectre de fluctuation de $V_{f}$ se trouve à fréquence nulle. Avec une diminution de cette résistance, la lorentzienne s'élargit et la contribution à la fréquence de transition $\Delta E / \hbar$ du qubit augmente tandis que celle à fréquence nulle doit diminuer. Une grande résistance $\operatorname{Re}[Z(\omega)]$ pour la jonction d'interaction favorise donc un grand temps de relaxation $\Gamma_{r}^{-1}$ mais tend à diminuer le temps de déphasage $\Gamma_{\phi}^{*-1}$. La figure 4.7 présente ces temps. On remarque, comme on s'y attend pour une grande résistance, que le temps de relaxation est beaucoup plus grand que le temps de déphasage. De plus, le temps de relaxation diminu à la dégénérescence $n_{g}=1 / 2$ puisqu'à ce point $\theta=\pi / 2$ et le bruit - est selon $x^{\prime}$ seulement. En retour, il n'y a pas de bruit selon $z^{\prime}$ et le déphasage diverge. De la même façon, lorsque $\Phi_{x} / \Phi_{0}=1 / 2$, la composante du bruit selon $x^{\prime}$ s'annule et le temps de relaxation (déphasage) est grand (petit). Ces taux de décohérence semblent suffisamment longs pour permettre l'observation d'oscillations cohérentes entre le qubit et la jonction. 


\subsection{INTERACTION RÉSONANTE POUR QUBITS SUPRACONDUCTEURS}

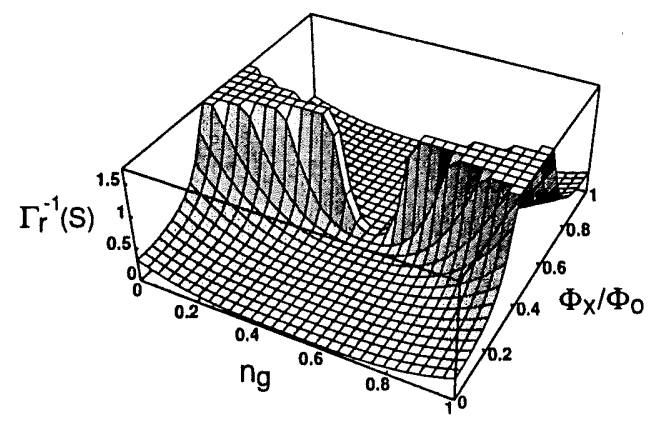

(a)

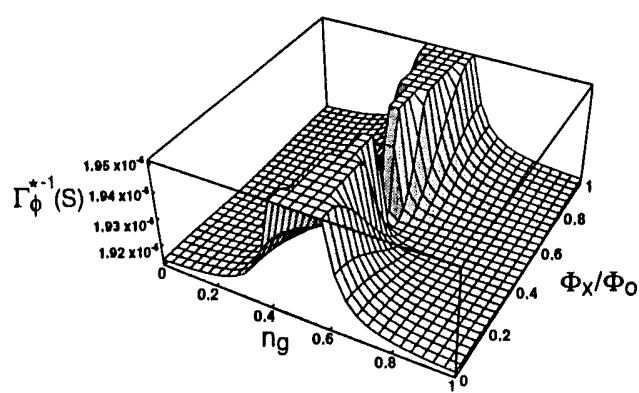

(b)

Fig. 4.7: Temps de relaxation (a) et de déphasage (b) d'un qubit de charge en présence d'une CBJJ en fonction de la charge de grille et du flux extérieur sur le qubit.

Pour les qubits phase-charge, on travaille au point d'opération $\left(n_{g}=1 / 2, \Phi=\right.$ $0)$. Dans l'approximation à deux niveaux, l'hamiltonien système-bain prend alors la forme $H_{S B}=e V_{f}\left(C_{c} / C_{\Sigma}\right) \tau_{x}$. Dans ce modèle, il n'y donc pas de déphasage pour ces qubits. Les fluctuations de voltage à la fréquence de transition $\Delta E / \hbar$ peuvent toutefois induire des transitions non-voulues et donc de la relaxation. En choisissant, comme précédemment $\operatorname{Re}[Z(\omega)]$ grand on minimise toutefois les fluctuations à hautes fréquences et par conséquent la relaxation. Les qubits phase-charge semblent donc particulièrement bien adaptés au type d'interaction qubit-qubit suggéré ici.

Si l'on tient compte de la présence de deux qubits connectés à la jonction, on peut alors répéter le calcul précédent en tenant compte de la renormalisation des capacités. De même, dans ce cas, les fluctuations de voltage sur la paire de qubits sont corrélées. Les qubits sont alors couplés au même environnement (en omettant à nouveau l'environnement propre à chaque qubit). Dans une telle situation, les techniques de correction d'erreurs sont plus difficiles à appliquer. Toutefois, il s'agit d'un cas où les DFS peuvent être très utiles.

En effet, si les fluctuations se couplent en $\sigma_{z}$ au qubit, alors le code

$$
\begin{aligned}
& \left|0_{L}\right\rangle=|01\rangle \\
& \left|1_{L}\right\rangle=|10\rangle,
\end{aligned}
$$

déjà présenté à la section $\S 1.5 .3$, protégera la paire de qubits. Dans le cas où les fluc- 


\subsection{INTERACTION RÉSONANTE POUR QUBITS SUPRACONDUCTEURS}

tuations de voltage se couplent en $\sigma_{x}$, le code (1.31) est toutefois inutile. C'est le cas des qubits phase-charge puisque pour ceux-ci les états logiques sont les états propres. Pour déterminer le code à utiliser dans cette circonstance, il suffit de remarquer que, dans la notation de la section 1.5.2, l'opérateur du système se couplant au bain est ici $S_{\alpha}=\sigma_{x 1}+\sigma_{x 2}$. Puisque l'espace noyau de $S_{\alpha}$ satisfait par définition (1.28), il ne reste plus qu'à trouver les vecteurs appartenant à cet espace. De cette façon, on obtient le code

$$
\begin{aligned}
& \left|0_{L}\right\rangle=\frac{1}{\sqrt{2}}(|00\rangle-|11\rangle) \\
& \left|1_{L}\right\rangle=\frac{1}{\sqrt{2}}(|01\rangle-|10\rangle) .
\end{aligned}
$$

En utilisant deux qubits physiques par qubit logique, on peut donc protéger l'information des fluctuations collectives de voltage.

Si $n$ qubits physiques sont couplés à la même CBJJ, il est alors possible d'utiliser un des codes mentionnés ici dans le but d'obtenir $n / 2$ qubits logiques. Notons que dans la situation où le code (4.47) est utilisé, il est possible d'utiliser les résultats de la section $\S 1.5 .3$ et une modification de ceux de la référence [185] afin de faire du calcul universel sur les qubits logiques. En effet, de l'équation (1.32) on sait que l'hamiltonien $\sigma_{z}$ appliqué sur un des qubits physiques agit comme $\bar{\sigma}_{z}$ sur le code. $\mathrm{Si}$ l'hamiltonien à deux qubits est du type XY, alors, comme on l'a déjà montré en (1.30), celui-ci permet de réaliser $\bar{\sigma}_{x}$ sur le code. Tel que vu à la section $\S 4.3 .2$, dans le cas où un qubit phase-charge interagit avec une CBJJ, l'hamiltonien d'interaction est plutôt du type $H_{ \pm}$. Il est simple de vérifier que ce dernier agit comme $\bar{\sigma}_{y}$ sur le code. On en déduit donc que, accompagné de l'hamiltonien à un bit physique $\sigma_{z}$, une ou l'autre de ces interactions permettent de réaliser $\overline{S U(2)}$ sur le sous-espace logique.

Afin de compléter la preuve d'universalité, il reste à obtenir un terme d'interaction non-trivial sur le sous-espace du code. Dans le cas du modèle XY, ceci a déjà été obtenu à la référence [185]. On simplifie ici ces résultats et les généralise au cas $H_{ \pm}$à l'aide des égalités suivantes :

$$
\begin{aligned}
e^{-i\left(\sigma_{x 1} \sigma_{x 3}+\sigma_{y 1} \sigma_{y 3}\right) \pi / 2} H_{X Y 23} e^{+i\left(\sigma_{x 1} \sigma_{x 3}+\sigma_{y 1} \sigma_{y 3}\right) \pi / 2} & =-i \sigma_{z 2} \sigma_{z 3} \cdot H_{X Y 12} \\
e^{-i\left(\sigma_{+1} \sigma_{-3}+\sigma_{-1} \sigma_{+3}\right) \pi / 2} H_{ \pm 23} e^{+i\left(\sigma_{-1} \sigma_{+3}+\sigma_{-1} \sigma_{+3}\right) \pi / 2} & =-\sigma_{z 2} \sigma_{z 3} \cdot H_{X Y 12}
\end{aligned}
$$


Ces relations signifient que l'interaction entre les qubits physiques 2 et 3 précédée et suivie de pulses d'interaction $\pi / 2$ entre les qubits physiques 1 et 3 agit comme $\sigma_{z 2} \sigma_{z 3} \cdot H_{X Y 12}$ sur les qubits physiques. De la même façon qu'à la section $\S 4.3 .1$, on réussit donc à simuler un hamiltonien par un autre. Cette simulation est intéressante puisque, comme il est simple de le vérifier, $\sigma_{z 2} \sigma_{z 3} \cdot H_{X Y 12}$ agit comme $-i \overline{\sigma_{y} \sigma_{z}}$ sur le code. Puisque cet hamiltonien permet d'enchevêtrer une paire de qubits logiques, ceci complète la preuve d'universalité.

Quelques remarques sont de mise ici. Premièrement, les pulses $\pi / 2$ font interagir des qubits qui ne sont pas premiers voisins. Ceci ne devrait pas causer de problèmes lorsque les qubits sont connectés à une même CBJJ. En second lieu, il est important de mentionner qu'il n'est pas possible en pratique de coupler un nombre arbitrairement grand de qubits à une même jonction. En présence d'un grand nombre de qubits, il peut en effet être difficile que chaque qubit ait une séparation en énergie $\Delta E$ suffisamment différente de celle des autres. De plus, on remarque que lors des opérations $\bar{\sigma}_{x}$ et $\overline{\sigma_{y} \sigma_{z}}$ décrites ci-haut il est nécessaire de transférer l'information d'au moins un qubit vers la jonction afin de le faire interagir indirectement avec un second qubit. Le système sort donc du sous-espace logique au cours des manipulations pour y revenir seulement à la fin de l'opération. Lors de ces manipulations, les qubits ne sont donc pas protégés des fluctuations. Ce désavantage n'est toutefois pas seulement dû au fait qu'une jonction intermédiaire est utilisée pour l'interaction. En effet, on vérifie facilement que le premier pulse $\pi / 2$ de la séquence (4.49) fait sortir le système du sous-espace logique et ce n'est que suite au second pulse $\pi / 2$ que celui-ci y retourne. Ainsi, même si les qubits interagissaient directement (grâce à une interaction capacitive par exemple) ce problème persisterait. Cette sortie hors du sous-espace logique intervient aussi pour la séquence plus complexe décrite à la référence [185]. Ceci n'a toutefois pas été relevé dans cette dernière publication. Finalement, il serait intéressant de vérifier s'il est possible de faire du calcul universel sous le code (4.48).

\subsection{Sommaire}

La méthode d'interaction qubit-qubit présentée dans ce chapitre a l'avantage d'être ajustable : l'interaction peut être 'ouverte' ou 'fermée' au besoin. Grâce au "re- 
couplage", cette approche est universelle. De même, elle peut être utilisée pour faire interagir des qubits de différents types. Un avantage important de cette approche est qu'elle conserve la possibilité de faire des opérations logiques en parallèle. Elle est donc compatible avec les techniques d'optimisation et du calcul tolérant aux imperfections. Finalement, l'interaction est ajustée en manipulant la jonction intermédiaire et non les qubits. Minimiser les manipulations sur les qubits est une bonne façon de minimiser les erreurs et de maximiser le temps de cohérence.

Un problème relié à cette approche est la fuite hors des états logiques de la jonction. Ceci peut être minimisé en opérant adiabatiquement sur le système. De même, des techniques algorithmiques ont été développées pour prévenir un phénomène similaire dans d'autres architectures d'ordinateurs quantiques $[169,186,187]$. Il serait intéressant d'adapter ces idées au système étudié ici.

Une seconde difficulté associée au modèle d'interaction présenté ici se trouve dans le fait que les opérations de la figure 4.6 doivent être réalisées très précisément. Dans le cas d'opérations imparfaites, il est possible qu'il subsiste de l'enchevêtrement résiduel entre les qubits et la jonction. Évidemment, la question de la précision est omniprésente en calcul quantique et ceci ne devrait pas être considéré comme un défaut majeur de cette approche. De plus, les techniques de correction d'erreurs, les DFS et le contrôle bang-bang peuvent en principe aider à réduire ce type d'erreurs.

Afin d'aller plus loin dans cette étude, une recherche des valeurs optimales est nécessaire pour les différents paramètres comme les capacités et énergies Josephson. De même, le problème de la fuite hors des niveaux logiques de la jonction doit être étudié plus à fond.

Après avoir complété l'analyse présentée ici, des idées similaires ont été publiées par d'autres auteurs [188]. Ceux-ci utilisent un mode d'opération légèrement différent. En exploitant l'analogie entre ce système et les cavités électrodynamiques, ceux-ci suggèrent d'utiliser les modes résonant et dispersif de l'hamiltonien Jaynes-Cummings. Le mode résonant de cet hamiltonien n'est rien d'autre que ce que nous avons considéré ici. On retrouve le mode dispersif de cet hamiltonien lorsque la différence $\delta$ entre les niveaux d'énergie des systèmes couplés est beaucoup plus grande que l'intensité $\gamma$ de l'interaction : $\delta \gg \gamma$ [189]. L'avantage de ce mode d'opération est que, à moins d'imperfections dans les manipulations, il n'y a jamais simultanément deux quanta 


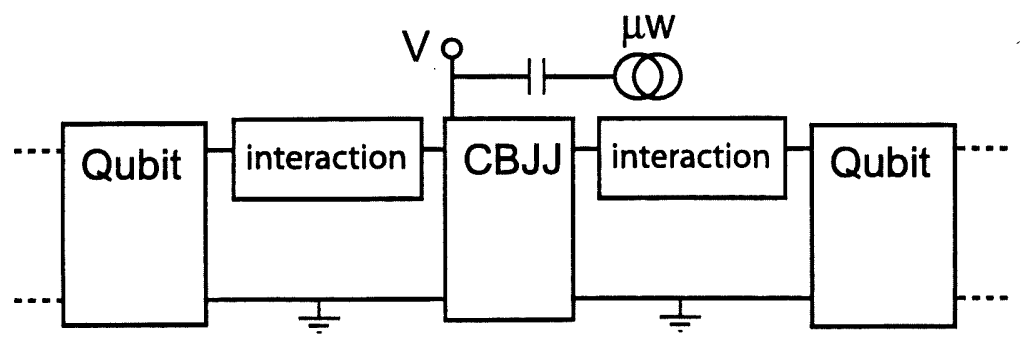

FIG. 4.8: Chaque qubit est couplé à une paire de CBJJ. L'interaction entre un qubit et une jonction peut être de nature capacitive, tel que décrit plus haut. La CBJJ est contrôlée par un courant continu et par des impulsions de courant alternatif $(\mu w)$. Pour la lecture d'un qubit, on permute l'état du qubit et de la jonction puis on mesure la jonction. Le voltage $V$ à ces bornes est le résultat.

dans la cavité (jonction). Dans l'approche décrite ici, qui n'est basée que sur le mode résonant, si les deux atomes (qubits) sont initialement dans l'état $|1\rangle$, alors à un moment des opérations la cavité se retrouve dans l'état $|2\rangle$ (suite à l'impulsion $\pi / 2$ à la figure 4.6). Ceci ouvre la porte à la fuite hors des états logiques. Quoique le mode d'opération suggéré à la référence [188] semble intéressant, certains aspects restent toutefois à étudier. En particulier, même s'il semble minimisé, le problème de la fuite n'a pas été abordé dans cette publication. De même, le passage entre les modes dispersif et résonant devrait être analysé.

D'autres auteurs ont aussi étudié l'interaction de différents circuits supraconducteurs et d'un circuit $L C$ [190-193]. Ceux-ci ne s'intéressaient toutefois pas à l'interaction entre qubits. Plus récemment et en se basant sur les travaux présentés ici, le groupe du Maryland a étudié en plus de détails la structure des niveaux d'énergie d'une paire de CBJJ couplée capacitivement [194].

Finalement, il est intéressant de remarquer que les jonctions de couplage peuvent en principe servir d'appareil de mesure [195]. En effet, après avoir effectué la première impulsion $\pi$ de la figure 4.6, l'état du qubit et celui de la jonction ont été échangés. - La lecture de la jonction correspond alors à une mesure de l'état du qubit. Cette remarque suggère l'utilisation d'un design intégré où les jonctions jouent le rôle d'intermédiaire pour l'interaction qubit-qubit et d'appareil de mesure. Cette architecture est présentée à la figure 4.8. Un problème de ce design est que, lors de la mesure d'une 
jonction sous-amortie, un voltage DC apparait à ses bornes. Ainsi, les qubits voisins du qubit mesuré verront ce voltage et subiront un déphasage en conséquence. À moins de mesurer simultanément tous les qubits ou de pouvoir filtrer ce pulse de voltage, cette approche intégrée ne semble pas avantageuse. Puisqu'une paire de qubits voisins verra (dans le cas idéal) une même impulsion de voltage, il semble toutefois possible d'utiliser une approche algorithmique à ce problème. En effet, en encodant un qubit logique à l'aide d'une paire de qubits physiques, comme en (4.47) ou (4.48), le qubit logique est insensible au fluctuations de voltage. L'utilisation des codes dans ce contexte mérite d'être étudiée en plus de détails.

La méthode d'interaction qubit-qubit proposée dans ce chapitre repose sur des composantes ayant, séparément, fait leur preuve expérimentalement. Néanmoins, faire fonctionner ensemble et de façon cohérente ces composantes pose un défi expérimental important. Il semble toutefois qu'un tel défi soit incontournable pour le domaine. Les résultats principaux de ce chapitre font l'objet d'une publication [179]. 


\section{Chapitre 5}

\section{Lecture d'un qubit de phase supraconducteur}

No elementary quantum phenomenon is a phenomenon until it is a registered phenomenon.

- John A. Wheeler

Le paradigme standard de l'informatique quantique, tel que présenté à la section $\S 1.2$, peut être assoupli de diverses façons. Un des critères qui ne semble toutefois pas pouvoir être éliminé est celui de la mesure. Afin d'être utile, il sera nécessaire de lire l'information de l'ordinateur. Comme nous l'avons vu dans le contexte de la correction d'erreurs quantiques, un appareil de mesure devrait pouvoir rapidement et efficacement lire les qubits. Idéalement, cette mesure devrait être de type projective. Une mesure faible sur un ensemble, comme en RMN de l'état liquide, peut aussi convenir $[73,74]$.

Dans ce chapitre, on s'intéresse à une nouvelle approche pour la mesure d'un qubit supraconducteur de phase. Avant de s'intéresser à ces résultats, on commence par présenter certains concepts utiles concernant la théorie de la mesure. Le but n'est pas de se pencher sur les problèmes d'interprétation ou de philosophie reliés au problème de la mesure en mécanique quantique. Une vaste littérature existe sur ces questions et le lecteur intéressé y est référé [196-200]. L'approche utilisée ici est plus pragmatique. On s'intéresse à des appareils de mesure réels et donc non idéaux. On 


\subsection{ASPECTS DE LA MESURE EN MÉCANIQUE QUANTIQUE}

cherchera aussi à préciser les termes 'rapidement' et 'efficacement' utilisés dans le paragraphe précédent.

\subsection{Aspects de la mesure en mécanique quantique}

Dans l'approche de von Neumann [201], afin de mesurer une observable $Q$ du système $\mathcal{S}$ initialement dans l'état $c_{0}\left|\psi_{0}\right\rangle+c_{1}\left|\psi_{1}\right\rangle$, on couple cette observable à un 'pointeur' $M$. Ce dernier sert d'appareil de mesure. Initialement, le pointeur et le système à mesurer ne sont pas enchevêtrés :

$$
\left(c_{0}\left|\psi_{0}\right\rangle+c_{1}\left|\psi_{1}\right\rangle\right)|m\rangle
$$

où $|m\rangle$ est l'état initial du pointeur. L'interaction système-pointeur enchevêtre les deux systèmes :

$$
\left(c_{0}\left|\psi_{0}\right\rangle+c_{1}\left|\psi_{1}\right\rangle\right)|m\rangle \longrightarrow c_{0}\left|\psi_{0}\right\rangle\left|m_{0}\right\rangle+c_{1}\left|\psi_{1}\right\rangle\left|m_{1}\right\rangle
$$

Afin qu'il nous soit possible de distinguer les états du pointeur, celui-ci doit être 'macroscopique'. De ce fait, le pointeur interagit fortement avec l'environnement. En tenant compte de cette interaction, l'état du système total est donc plutôt de la forme

$$
|\Psi\rangle=c_{0}\left|\psi_{0}\right\rangle\left|m_{0}\right\rangle\left|e_{0}\right\rangle+c_{1}\left|\psi_{1}\right\rangle\left|m_{1}\right\rangle\left|e_{1}\right\rangle
$$

où $\left|e_{i}\right\rangle$ est l'état de l'environnement.

Puisque qu'il est impossible, en pratique, de contrôler et mesurer chacun des degrés de liberté de l'environnement, la description du système du point de vue de l'observateur est plutôt

$$
\rho_{S M}=\operatorname{Tr}_{E} \rho_{S M E}
$$

Si $\left\langle e_{0} \mid e_{1}\right\rangle=0$, l'enchevêtrement entre $\mathcal{S}$ et le détecteur est réduit à des corrélations classiques; $\rho_{S M}$ n'a pas d'éléments hors-diagonaux. Si $\left|m_{0}\right\rangle$ et $\left|m_{1}\right\rangle$ sont distinguables - expérimentalement, une mesure du pointeur nous informe alors sur l'état du système. Il est intéressant de remarquer que ce résultat est obtenu sans faire appel au 'postulat de réduction' de la mécanique quantique.

Dans le contexte du calcul quantique, on cherche à projeter les qubits dans la base de calcul et a connaître le résultat de cette projection. Puisque la base de calcul 
est formée des états propres de $\sigma_{z}$, c'est cette cette dernière observable que l'on doit mesurer. Toujours dans l'approche de von Neumann, on peut alors écrire l'hamiltonien qubit-appareil de mesure sous la forme

$$
H=-\frac{1}{2} B_{x} \sigma_{x}-\frac{1}{2} B_{z} \sigma_{z}+\eta \sigma_{z} M+H_{M}
$$

où $\eta$ représente l'amplitude de l'interaction et $H_{M}$ l'hamiltonien interne de l'appareil de mesure, incluant l'interaction à l'environnement. Afin de simplifier la description, on a supposé ici que le qubit n'est couplé à l'environnement que par son interaction avec $M$.

Lorsqu'il n'y a pas de mesure en cours, la constante de couplage $\eta$ doit s'annuler. À l'opposé, au moment de la mesure, la constante de couplage doit être très grande de sorte que l'hamiltonien d'interaction $\eta \sigma_{z} M$ domine complètement $H$. Dans ce cas, on a $\left[H, \sigma_{z}\right] \approx 0$ et $\sigma_{z}$ est une constante du mouvement. Il s'agit d'une situation idéale puisque l'appareil de mesure ne perturbe alors pas l'observable à mesurer. Cette supposition était implicite dans (5.2). Évidemment, la mesure en mécanique quantique perturbe toujours le système et la situation décrite ici n'échappe pas à cette règle. Ici, $\sigma_{x}$ n'est pas une constante du mouvement et ses états propres sont perturbés par l'interaction avec le détecteur.

Pour les systèmes de l'état solide, ce modèle n'est certainement pas réaliste. L'appareil de mesure sera vraisemblablement microfabriqué à proximité du (des) qubit(s) et ne pourra être physiquement déconnecté de celui-ci (ceux-ci). La constante de couplage ne s'annulera donc probablement pas exactement lorsqu'il n'y a pas de mesure en cours. De même, au moment de la mesure, le couplage $\eta$ ne peut être arbitrairement grand de sorte que $\left[H, \sigma_{z}\right] \approx 0$ ne sera vraisemblablement pas une bonne approximation. Dans ce cas, $\sigma_{z}$ n'est pas une constante du mouvement et l'interaction entre le qubit et l'appareil de mesure aura pour effet de 'mélanger' les états du qubit (modifier les coefficients $c_{0}$ et $c_{1}$ ). Finalement, le pointeur peut aussi déphaser le qubit sans pour autant effectuer de mesure. Une telle situation se présente si $\left\langle e_{0} \mid e_{1}\right\rangle=0$ mais $\left|m_{0}\right\rangle=\left|m_{1}\right\rangle$ dans (5.3).

En pratique, on cherchera donc un détecteur ne diminuant pas le temps de cohérence du qubit au-delà de son temps de cohérence 'intrinsèque' (en l'absence du pointeur). De même, le temps que prend la mesure à être complétée $t_{m e s}$, devrait être 


\subsection{ASPECTS DE LA MESURE EN MÉCANIQUE QUANTIQUE}

plus court que le temps typique de mélange $t_{m i x}$ après lequel les populations de la. matrice densité du qubit atteignent leur valeur à l'équilibre. En dernier lieu, comme on le voit en (5.4), une mesure idéale déphase complètement le qubit. On doit donc avoir $t_{m e s} \geq t_{\phi}$, où $t_{\phi}$ est le temps de déphasage en présence d'interaction avec le pointeur. On a donc les inégalités suivantes entre ces trois temps caractéristiques

$$
t_{m i x} \gg t_{m e s} \geq t_{\phi}
$$

On reviendra en plus de détails sur ces temps à la section suivante.

Lorsque le terme d'interaction $\eta \sigma_{z} M$ ne domine pas l'hamiltonien total, la symétrie de l'hamiltonien du qubit est très importante. En particulier, si $B_{x}$ est non-nul comme en (5.5), $\sigma_{z}$ n'est pas une constante du mouvement. L'interaction perturbe alors l'observable que l'on cherche à mesurer, diminuant le temps de mélange. Une telle situation n'est pas utile pour le calcul quantique. Dans le cas où le couplage est faible, ce type de 'mesure faible' peut toutefois fournir de l'information intéressante sur le qubit et son environnement. En effet, l'appareil de mesure perturbe le qubit qui répond à sa fréquence naturelle $\omega_{0}=\sqrt{B_{x}^{2}+B_{z}^{2}} / \hbar$. La densité spectrale du bruit à la sortie de l'appareil de mesure présentera alors un pic à la fréquence d'oscillation cohérente du qubit . La largeur de ce pic contient de l'information sur l'amplitude des oscillations et sur le taux de décohérence $[202,203]$. Ce type de mesure constitue donc un outil intéressant pour déterminer la 'qualité' d'un qubit. Notons aussi que, dans le même contexte, le point de vue inverse peut être adopté. Il est possible d'utiliser un qubit comme analyseur de bruit [134].

Une situation particulièrement intéressante pour le calcul quantique est lorsque $B_{x}=0$. Dans ce cas, $\sigma_{z}$ est une constante du mouvement même si le terme d'interaction ne domine pas l'hamiltonien total. Il s'agit d'une mesure idéale puisque l'action du détecteur est alors seulement de déphaser le qubit, sans introduire de mélange. On dispose alors du temps voulu pour réaliser la mesure avec la précision voulue. Ce type de mesure est connu sous le nom de 'Quantum Non-Demolition Measurement' (QND) $[204,205]$. 


\subsection{AMPLIFICATEURS LINÉAIRES}

\subsection{Amplificateurs linéaires}

Lorsque l'appareil de mesure opère dans le régime linéaire, on peut facilement obtenir des résultats pour les temps caractéristiques introduits à la section précédente [203, $206,207]$. Dans cette section, on suit de près la section $\S 5 . H$ de la référence [108].

Comme à la section précédente, on suppose le pointeur $M$ couplé à l'observable $Q$ du système par un terme d'interaction $Q M$. Dans cette approche, $Q$ joue le rôle de signal d'entrée auquel correspond un signal $V$ à la sortie du détecteur. La fonction de réponse $\lambda=d\langle V\rangle / d Q$ décrit la variation du signal de sortie due à une variation du signal d'entrée. Dans le cas de la mesure d'un qubit, le signal d'entrée est proportionnel à $\sigma_{z}$ et l'hamiltonien d'interaction est $\eta \sigma_{z} M$ comme précédemment.

Dans une situation QND, le détecteur ne mélange pas les états du qubit mais introduit un déphasage. De la même façon qu'à la section $§ 3.3$, ce taux est donné par

$$
t_{\phi}^{-1}=\frac{\eta^{2}}{\hbar^{2}} \frac{B_{z}^{2}}{B_{z}^{2}+B_{x}^{2}} S_{M}(\omega \rightarrow 0)
$$

où $S_{M}$ est la densité spectrale des fluctuations de $M$ sans la présence du qubit. Dans le cas QND, on a simplement $B_{x}=0$ dans cette dernière relation.

En raison de l'interaction $\eta \sigma_{z} M$, les états logiques du qubit produisent des signaux de sortie différents $V_{ \pm}=V_{0} \pm \Delta V / 2$. L'écart entre ces signaux est donné par $\Delta V=2 \eta \lambda$. En réponse linéaire, l'écart entre les distributions de probabilité correspondant aux deux états logiques sera, typiquement, $2 \eta \lambda t$ après un temps $t$. De même, en supposant que les fluctuations $S_{V}$ du signal de sortie $V$ sont gaussiennes, ces distributions auront une largeur qui augmente comme $\sqrt{t S_{V}(\omega \rightarrow 0)}$. En exigeant que la séparation entre ces distributions soient plus grandes que leur largeur, on obtient le temps de mesure à partir duquel il est en principe possible de distinguer les deux valeurs logiques [207]

$$
t_{m e s}^{-1}=\frac{\Delta V^{2}}{4 S_{V}(\omega \rightarrow 0)}
$$

De ces résultats, on obtient le ratio suivant entre $t_{m e s}$ et $t_{\phi}$

$$
\frac{t_{m e s}}{t_{\phi}}=\frac{S_{M} S_{Q}}{\hbar^{2}}
$$

où $S_{Q} \equiv S_{V} / \lambda^{2}$ est le bruit qu'il faut introduire au signal d'entrée afin d'obtenir le bruit $S_{V}$ à la sortie. Les densités spectrales $S_{M}$ et $S_{Q}$ respectent une inégalité de 


\subsection{DÉTECTEURS POUR QUBITS SUPRACONDUCTEURS}

Heisenberg $S_{M} S_{Q} \geq \hbar^{2}[204,206]$. En portant cette inégalité dans (5.9), on retrouve la contrainte $t_{m e s} \geq t_{\phi}$ introduite à la section précédente.

Dans la situation non QND où $B_{x} \neq 0$, le détecteur induit aussi le mélange des états du qubit. Ce processus se fait à un taux

$$
t_{m i x}^{-1}=\frac{\eta^{2}}{\hbar^{2}} \frac{B_{x}^{2}}{B_{z}^{2}+B_{x}^{2}} S_{M}\left(\omega_{0}\right) .
$$

Le ratio $t_{\phi} / t_{m e s}$ est un indicateur de l'efficacité du détecteur. Un détecteur idéal aura $t_{\phi} / t_{m e s}=1$. Dans le cas où $t_{\phi} / t_{m e s}<1$, le détecteur permet de distinguer les états du qubit après un temps plus long qu'il ne le serait possible en principe $[108,206]$. D'autres quantités, comme la sensibilité en énergie, sont utiles pour caractériser les performances d'un détecteur. Ceux-ci ne seront pas présentés ici et le lecteur est référé à la littérature sur le sujet $[108,203,204,206]$.

\subsection{Détecteurs pour qubits supraconducteurs}

Dans cette section, on commence par présenter les concepts importants concernant la mesure d'un qubit de phase à l'aide d'un SQUID-dc. Cet exemple fait intervenir plusieurs concepts qui seront utiles par la suite. On présente ensuite différentes stratégies parues dans la littérature pour la lecture des qubits supraconducteurs.

\subsubsection{Lecture du qubit de phase par un SQUID-dc}

Les SQUIDs sont bien connus pour leur grande sensibilité au champ magnétique. L'utilisation de SQUIDs pour la mesure de champs magnétiques est décrite en détail dans plusieurs références $[118,208-210]$. Compte tenu cette grande sensibilité, l'application à la lecture d'un qubit de phase semble naturelle. Ceci a été étudié, entre autres, aux références $[125,135,136,203,211,212]$.

Un SQUID-dc a été utilisé comme détecteur pour l'expérience déjà cité à la section $\$ 3.4$ sur le qubit à trois jonctions [156]. Dans cette approche, le qubit est couplé inductivement au SQUID, tel que présenté à la figure 5.1. La mesure est basée sur le fait que le flux généré par le qubit change l'énergie Josephson effective du SQUID. En retour, ceci modifie sa caractéristique courant-voltage. Pour faire la mesure, on 


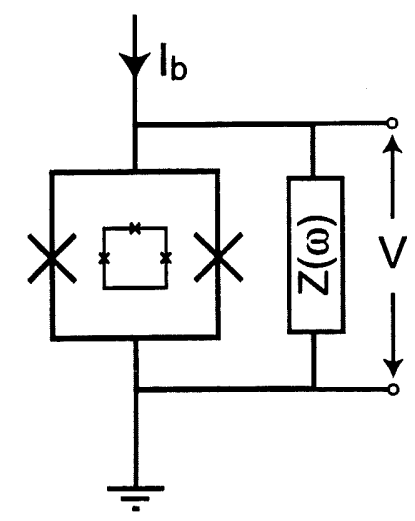

(a)

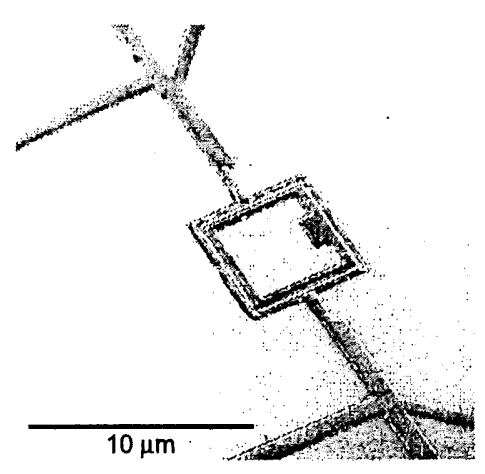

(b)

FIG. 5.1: Qubit de phase couplé inductivement à un SQUID-dc. a) Un courant $I_{b}$ est appliqué dans le SQUID. La valeur du courant auquel apparaît un voltage aux bornes du SQUID contient de l'information sur l'état du qubit. L'impédance $Z(\omega)$ est cruciale dans la détermination des caractéristiques du détecteur et sa rétroaction sur le qubit. b) Image par microscopie électronique du système utilisé pour l'expérience décrite à la référence [156]. Le qubit et le détecteur sont faits d'aluminium. Cette image est tirée du site web du groupe de Delft (http ://qt1.tn.tudelft.nl/ caspar/).

applique donc un courant $I_{b}$ dans le SQUID et on enregistre la valeur de ce courant pour lequel le système passe à l'état résistif.

Pour déterminer la qualité et la rétroaction de ce détecteur, la résistance de shunt $R_{s}$ aux bornes du SQUID est cruciale. Dans le cas où $R_{s} \gg R_{K} \sqrt{E_{C} / E_{J}}$, avec $R_{K}=h / 4 e^{2}$ le quantum de résistance, la courbe IV du SQUID est hystérétique [118]. Cette situation est présentée à la figure 5.2. Il s'agit du régime sous-amorti ou 'unshunted'. Dans ce régime, le passage à l'état résistif se fait à un courant de commutation $I_{s w}$ inférieur au courant critique $I_{c}$. L'avantage du cas sous-armorti est que le SQUID n'injecte que peu de bruit dans le qubit lorsqu'il n'y a pas de mesure en cours. Malheureusement, dans ce régime, le passage à l'état résistif est un processus aléatoire [118]. Si le qubit est dans l'état $|i\rangle$, on peut donc s'attendre à observer la commutation dans une plage de courants $\Delta I_{s w}$ centrée autour d'une valeur $I_{s w}^{i}$. Lorsque cette distribution est trop grande par rapport à la séparation $I_{s w}^{1}-I_{s w}^{0}$, seulement une mesure 


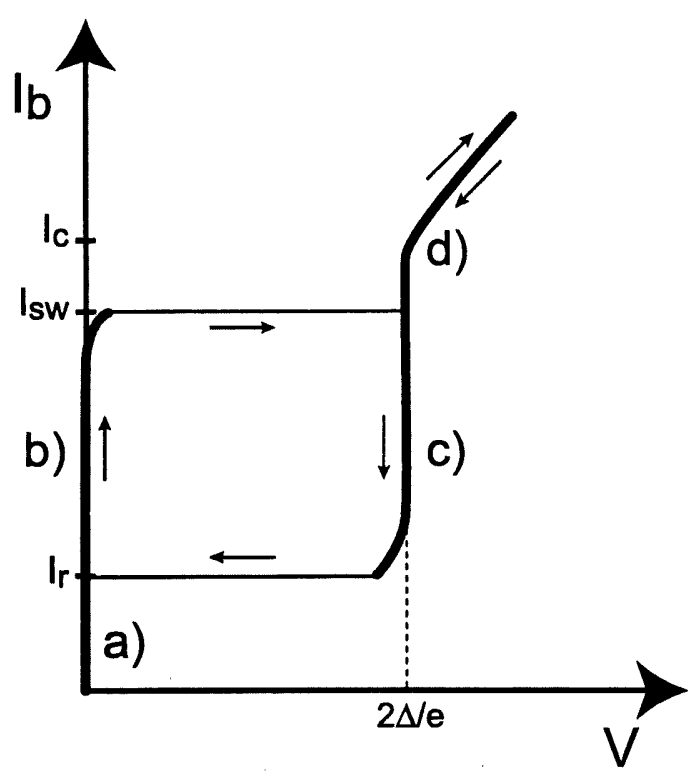

a)

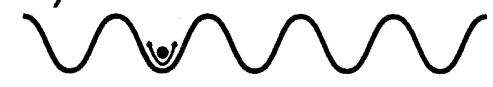

b)

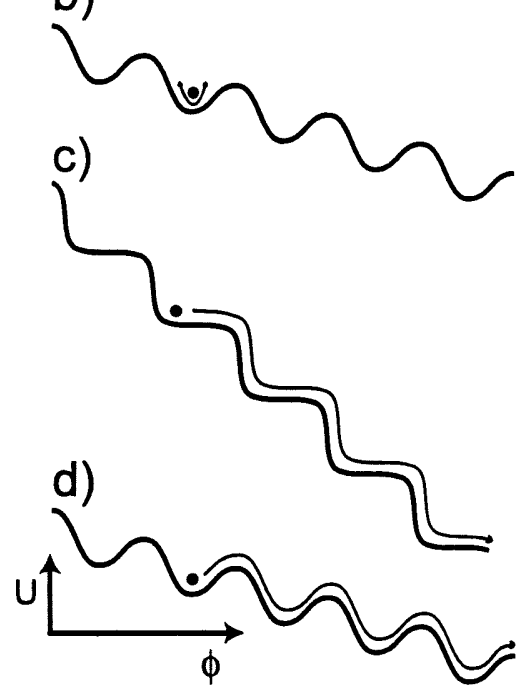

FIG. 5.2: Courbe IV hystérétique pour une jonction sous-armortie (gauche). Forme du potentiel $U$ de la jonction en fonction de la phase en différentes régions de la courbe IV (droite). a) Sans courant appliqué, la particule est localisée dans un des puits. Elle oscille à la fréquence plasma autour de sa position d'équilibre. b) Un courant extérieur ajoute un terme $-\Phi_{0} I_{b} \phi / 2 \pi$ au potentiel de la jonction. Si ce courant est beaucoup plus faible que le courant critique $I_{c}$, la particule reste localisée dans un puits. c) Lorsque $I_{b}=I_{c}$, il n'y a plus de barrière de potentiel et la moindre fluctuation amène la particule à dévaler la pente. Un voltage apparaît alors aux bornes de la jonction. Pour une jonction sous-amortie, le temps de relaxation $\tau=R C$ dans le puits est grand et la particule a une probabilité élevée de passer au-dessus de la barrière de potentiel (ou sous la barrière par effet tunnel) même lorsque $I_{b}<I_{c}$. La commutation vers l'état résistif est donc un processus aléatoire. d) Toujours pour le cas sous-amorti, la dissipation dans les puits est très faible et l'inertie de la particule est telle qu'elle ne se relocalisera pas avant un courant de repiègeage ('retrapping current') $I_{r}$ inférieur au courant critique. Dans le cas idéalisé où il n'y a pas de dissipation, le repiègeage s'effectue à courant nul. 


\subsection{DÉTECTEURS POUR QUBITS SUPRACONDUCTEURS}

faible du qubit est possible. Il est alors nécessaire d'accumuler des statistiques sur la mesure pour distinguer les états logiques. C'est ce qui a été fait dans l'expérience décrite à la référence [156].

Dans le régime sur-armorti, $R_{s} \ll R_{K} \sqrt{E_{C} / E_{J}}$, le SQUID n'est pas hystérétique et $I_{s w} \approx I_{c}$ [118]. Une mesure projective est alors en principe possible. Toutefois, dans ce régime, la rétroaction du détecteur sur le qubit est importante et déphase rapidement ce dernier, même lorsqu'il n'y a pas de mesure en cours.

Ainsi, malgré que le SQUID-dc soit très sensible au champ magnétique, il ne semble pas approprié, sans modifications, à la mesure d'un qubit de phase. Intuitivement, le problème est que pour effectuer une bonne mesure, le détecteur doit être dissipatif. Toutefois, afin d'être utile au calcul quantique, les qubits doivent être bien écrantés des fluctuations dues à ces éléments dissipatifs. Avec le SQUID-dc directement couplé au qubit, il semble difficile de faire cet écrantage lors des manipulations cohérentes. Notons que le régime $R_{s} \approx R_{K}$ peut toutefois être un compromis intéressant [211].

\subsubsection{Autres stratégies}

Pour la mesure des qubits de phase, peu approches alternatives au SQUID-dc ont été présentées jusqu'à présent. Notons toutefois la méthode de 'mesure d'impédance' de Il'ichev et al. [213]. Cette approche semble par contre plus appropriée pour déterminer les caractéristiques d'un qubit que pour faire une mesure projective de son état logique. Mentionnons aussi le 'INSQUID', étudié par la collaboration Berkeley-Karlsruhe [214]. Celui-ci agit comme intermédiaire entre le qubit et un SQUID-dc. En principe, cette approche permet de découpler le qubit du détecteur lorsqu'il n'y a pas de mesure en cours et ainsi de choisir les paramètres du SQUID-dc de façon à permettre la mesure projective. Ce découplage nécessite toutefois que les jonctions formant le INSQUID soient rigoureusement identiques. Si ce n'est pas le cas, la rétroaction du SQUID-dc sur le qubit sera non-nulle lors des manipulations cohérentes.

Pour les qubits de charges, différentes approches ont été suggérées. En particulier, la mesure de ce qubit par un transistor à un électron a été étudiée en détail par Shnirman et Schön [215]. L'avantage de cette approche est, qu'en raison du blocage de Coulomb, le qubit est écranté des fluctuations de la partie résistive du système 


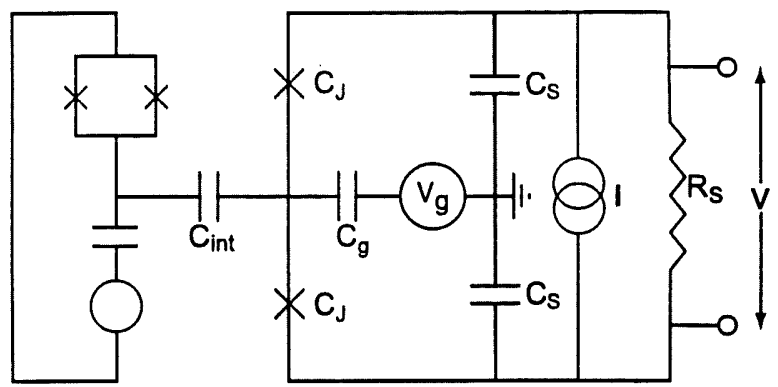

FIG. 5.3: Qubit de charge couplé capacitivement à un SSET. Le SSET agit comme une jonction Josephson ayant un courant critique modulé par le voltage sur l'île $I_{c}\left(n_{g}, n_{i}\right)$. Ici, $n_{g}$ correspond au voltage de grille et $n_{i}$ est la charge sur le qubit. En choisissant $R_{s}<R_{K}$, la jonction effective n'est pas hystérétique et le courant de commutation s'approche du courant critique. Une mesure du courant de commutation nous renseigne alors sur $n_{i}$.

lorsqu'il n'y a pas de mesure effectuée. Pour faire la mesure, le voltage de grille du transistor est réglé près de la dégénérescence et un voltage de transport est appliqué. La valeur logique du qubit change le voltage appliqué sur l'île du transistor et affecte ainsi le courant. Ce courant est la sortie du détecteur. Malheureusement, l'efficacité $t_{\phi} / t_{m e s}$ de ce détecteur est inférieure à l'unité. La situation peut être améliorée en ajustant la fabrication des jonctions tunnel du SET de sorte qu'elles aient des taux tunnel différents [108]. De plus, dans le régime où le 'cotunneling' est important. l'efficacité est plus grande mais l'intensité du signal de sortie est plus faible [216]. Un problème relié à l'application de ces approches est qu'aux températures auxquelles seront réalisées les expériences sur les qubits de charge, le transistor (typiquement fait d'aluminium) sera supraconducteur. Les caractéristiques du détecteur sont alors complètement différentes.

Le transistor à un électron supraconducteur (SSET) a été proposé comme électromètre par Zorin [217,218]. À très basse température, la sensibilité du SSET est supérieure à celle du SET. En fait, dans certains régimes, ce détecteur peut opérer en deçà de la limite standard quantique (SQL) [204]. Mentionnons que Zorin a aussi proposé un électromètre basé sur le transistor de Bloch couplé à un circuit $L C$ ayant un grand facteur de qualité [219]. Cette approche a des similitudes avec l'approche de Il'ichev et al. mentionnée au début de cette section [213]. Plus récemment, Zorin 
a suggéré un design de qubit incorporant un détecteur [220]. Ce design présente des similitudes avec le design phase-charge de Saclay $[6,126]$.

Le SSET a été étudié théoriquement et expérimentalement dans le contexte de la mesure d'un qubit de charge par Cottet et al. [141]. La figure 5.3 présente le système. Quoique cet électromètre soit en principe très sensible, le voltage à la sortie est très faible et doit être amplifié avec un amplificateur à basse température. Cottet et al. [141] (voir aussi [221]) ont utilisé une série de SQUID-dc comme amplificateur. L'amplificateur ajoute toutefois du bruit au signal de sortie de sorte que la sensibilité n'est pas aussi bonne que ce qu'elle pourrait être en principe. Le petit voltage de sortie semble donc être un problème pour ce type de détecteur.

Pour le qubit phase-charge de Saclay présenté à la figure 3.11, la mesure est réalisée en appliquant un flux $\Phi$ dans la boucle du qubit et un courant $I_{b}$ dans la jonction de lecture $E_{J 0}=\hbar I_{c 0} / 2 e$. Puisque le flux est non-nul, le système n'est plus au point d'opération privilégié et, selon l'état logique du qubit, un courant horaire $I_{q}$ ou anti-horaire $-I_{q}$ circule dans la boucle. Le courant total dans la jonction de lecture est alors $I_{b} \pm I_{q}$. Si $I_{b}$ est ajusté de façon à ce que $I_{b}+I_{q}>I_{c 0}$ mais $I_{b}-I_{q}<I_{c 0}$, on s'attend alors à mesurer un voltage aux bornes de la jonction seulement pour un des états logiques. En principe, l'efficacité de cette approche peut être très grande [126]. Dans l'expérience décrite à la référence [6], l'efficacité n'était toutefois pas suffisante pour réaliser des mesures du qubit 'en un coup'.

Rappelons finalement que l'approche décrite à la section $\S 4.4$ peut en principe être utilisée pour mesurer différents types de qubits.

\subsection{Lecture du qubit de flux.}

Dans cette section, on présente une approche originale appliquée à la mesure des qubits de flux supraconducteurs. Cette approche semble permettre la séparation nécessaire entre le système quantique cohérent et l'appareil de mesure dissipatif. La détection est basée sur un SSET couplé à un SQUID et agissant comme transducteur entre flux et charge. Le voltage aux bornes du SSET est la sortie du détecteur. Évidemment, cette approche souffre du problème discuté à la section précédente : ce voltage est très faible et peut être difficile à mesurer. Toutefois, le concept de 


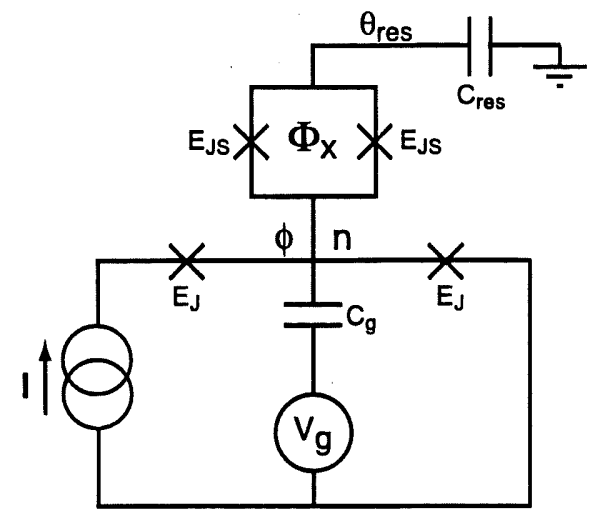

Fig. 5.4: Circuit utilisé par Matters et al. pour étudier l'effet des fluctuations de phase dans le régime de blocage de Coulomb. Le réservoir de phase est un supraconducteur massif. Figure adaptée de [222].

transducteur flux-charge pourra peut-être s'appliquer à un détecteur plus efficace en pratique. En guise d'exemple exploratoire, on considère tout de même le SSET ici.

Cette approche pour la mesure est basée sur des résultats expérimentaux du groupe de Delft [222]. On commence donc par présenter brièvement ces résultats pour ensuite s'intéresser au design du détecteur. On décrit ensuite des résultats illustrant son fonctionnement et ses performances.

\subsubsection{Contrôle des fluctuations phase-charge dans un SSET}

La figure 5.4 présente le design étudié par Matters et al. [222]. Un SSET est couplé à un supraconducteur massif par un SQUID. Le supraconducteur massif a une phase $\theta_{\text {res }}$ bien établie et celui-ci agit comme 'réservoir de phase' pour l'île du SSET. De plus, ce réservoir est connecté par une grande capacité $C_{r e s}$ à la masse de sorte qu'il n'y a pas de courant continu circulant dans le SQUID.

Avec ce circuit, il est possible de contrôler les fluctuations de charge $\Delta n$ et les fluctuations de phase $\Delta \phi$ de l'île du SSET. En effet, le voltage de grille offre un contrôle sur les fluctuations de charge de l'île. Comme on l'a déjà vu à la section $\S 3.2 .1$, on peut ajuster $n_{g}$ de sorte que la charge $n$ soit fixée (régime de blocage de Coulomb, $\Delta n=0)$ ou de façon à ce qu'un courant circule dans le SSET $(\Delta n>0)$. Ce contrôle sur les fluctuations de charge dans un SSET est bien connu [122,223]. L'aspect 
original du travail de Matters et al. vient du fait que le flux dans le SQUID permet un contrôle des fluctuations de phase. En effet, changer le flux dans le SQUID varie l'énergie Josephson effective du SQUID et, par le fait même, l'intensité du couplage entre l'île et le réservoir de phase. Lorsque le couplage entre ces éléments du circuit est important, la phase du réservoir tend à fixer la phase de l'île et donc à réduire $\Delta \phi$. Puisque la phase et la charge sont des variables conjuguées, leurs fluctuations ne sont pas indépendantes mais sont reliées par la relation d'incertitude $\Delta n \Delta \phi \geq 1[118,123]$. Par conséquent, fixer $\Delta \phi$ induit des fluctuations de charge sur l'île. De cette façon, il est possible d'avoir un courant à travers le SSET même lorsque celui-ci devrait être dans le régime de blocage de Coulomb.

Afin de confirmer ces attentes, Matters et al. ont mesuré le courant de commutation en fonction du voltage de grille et du flux dans le SQUID. Les résultats obtenus sont présentés à la figure 5.5. L'accord entre les résultats expérimentaux (points) et la théorie (ligne pleine) est très bon.

\subsubsection{Transducteur flux-charge pour qubits de flux}

Les résultats expérimentaux présentés à la section précédente montrent clairement que le courant critique d'un SSET peut être affecté par un flux. Toutefois, le SSET utilisé pour cette expérience était sous-amorti et la commutation un processus aléatoire. Depuis la publication de ces résultats, Zorin a montré que le SSET sur-amorti est, en principe, un électromètre efficace $[217,218]$. Dans cette section on combine ces concepts de transducteur flux-charge et d'électromètre efficace pour réaliser la lecture d'un qubit de phase.

La figure 5.6 présente cette idée appliquée au qubit à trois jonctions et au qubit basé sur les barrières de grain entre supraconducteurs de type $d$. Dans le premier cas, on couple inductivement le qubit au SQUID. Ceci déplace le point d'opération du détecteur et, par conséquent, affecte le courant critique. Dans le deuxième cas, le qubit prend la place du réservoir de phase. L'interaction entre la phase de l'île et celle du qubit change aussi le courant critique du détecteur.

Cette approche semble intéressante a priori puisque, dans le régime sur-amorti, le courant de commutation a une distribution très étroite. Une mesure 'en un coup' est 


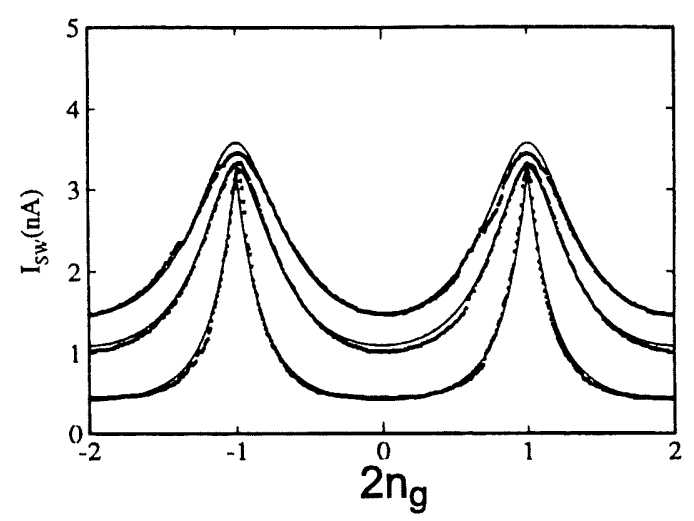

(a)

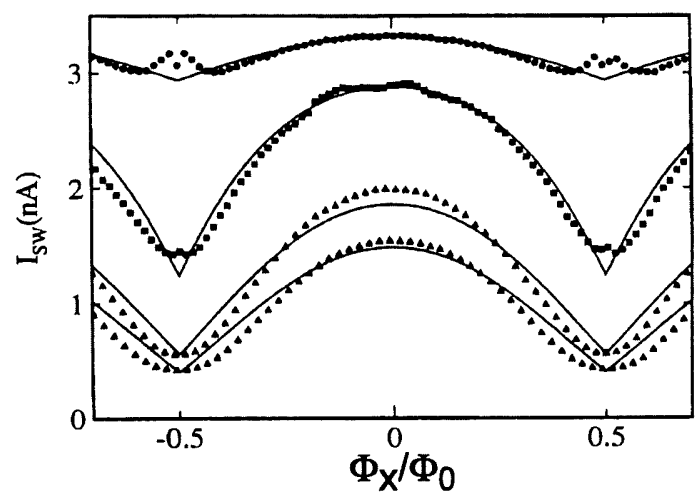

(b)

FIG. 5.5: a) Courant de commutation en fonction du voltage de grille pour différentes valeurs du flux $\Phi_{x}$ dans le SQUID $\left(\Phi_{x} / \Phi_{0}=0,0.25\right.$ et 0.5 de haut en bas). Les résultats expérimentaux (points) reproduisent bien les prédictions théoriques (lignes pleines). $\AA \Phi_{x}=$ 0 , le réservoir de phase est couplé fortement à l'île du SSET. Les fluctuations de phase sont supprimées et les résonances sont élargies. b) Courant de commutation en fonction du flux pour différentes valeurs de la charge de grille $\left(2 n_{g} / e=1,0.75,0.5\right.$ et 0 du haut vers le bas). Le courant de commutation est périodique en $\Phi_{x}$. Lorsque le voltage de grille est à la résonance $\left(2 n_{g} / e=1\right)$, les fluctuations de charge sont grandes et le flux a peu d'effets. Les énergies du circuit sont : $E_{J}=0.29 \mathrm{~K}, E_{C}=0.72 \mathrm{~K}$ et $E_{J S}=0.24 \mathrm{~K}$. Figure tirée de [222] 


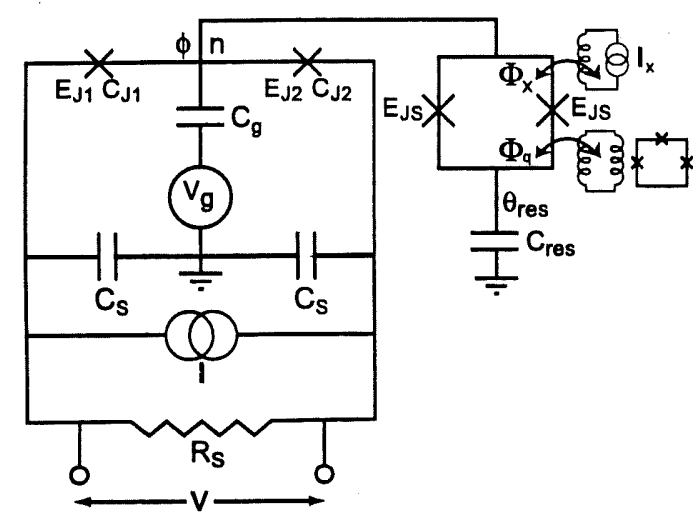

(a)

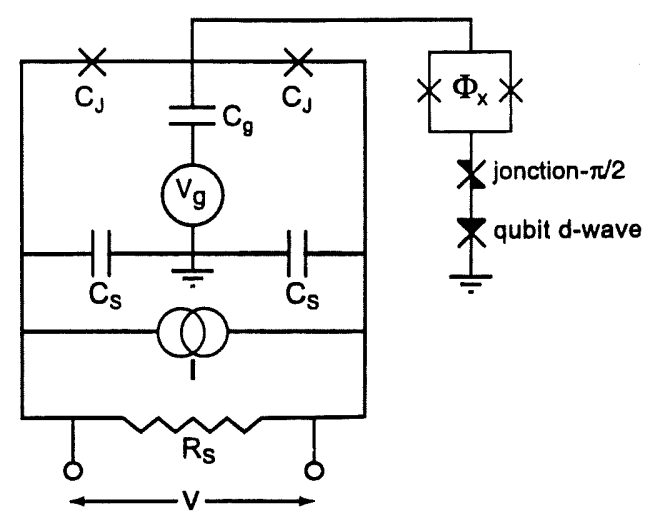

(b)

FIG. 5.6: a) SSET couplé à un réservoir de phase par un SQUID. Un qubit de flux est couplé inductivement au SQUID. Le flux généré par le qubit modifie le courant critique du SSET. Dans le régime sur-amorti, on peut en principe déterminer expérimentalement la valeur du courant critique. b) Lecture d'un qubit basé sur les supraconducteurs de type $d$. Le qubit remplace le réservoir de phase. De la même façon qu'en a), la phase du qubit modifie le courant critique du SSET. Dans ce cas, on doit ajouter une jonction $\pi / 2$ et les jonctions du SSET doivent être inégales afin d'avoir une influence mesurable du qubit sur le SSET. Cette situation ne sera pas étudiée dans le texte. Une analyse préliminaire a été présentée dans un rapport interne [224].

alors possible. De plus, comme il apparaîtra plus clairement par la suite, il est possible d'écranter le qubit des fluctuations dues à $R_{s}$ en choisissant le point d'opération $\left(n_{g}, \Phi_{x}\right)$ approprié. De même, puisque le SQUID n'est pas utilisé directement pour la mesure, celui-ci peut alors être dans le régime sous-amorti. Ainsi, le bruit dû au détecteur sur le qubit est minimisé en séparant la région dissipative de la région où la cohérence de phase doit être maintenue.

On s'intéressera ici au cas du qubit à trois jonctions. En suivant la procédure décrite à la section $\S 3.1$, on obtient pour l'hamiltonien de ce circuit :

$$
\begin{aligned}
H= & E_{C}\left(n-n_{g}\right)^{2}-E_{J 1} \cos (\phi-\theta / 2)-E_{J 2} \cos (\phi+\theta / 2) \\
& -2 E_{J S} \cos \frac{\pi}{\Phi_{0}}\left(\Phi_{x}+\Phi_{q}\right) \cos \left(\phi-\theta_{r e s}\right) .
\end{aligned}
$$

Dans cette expression, $n$ est la charge sur l'île du SSET et $\phi$ la phase conjuguée. 
$\theta$ est la différence de phase à travers le SSET et $\theta_{\text {res }}$ celle du réservoir de phase. L'énergie de charge $E_{C}=(2 e)^{2} / 2 C_{\Sigma}$ dépend de la capacité totale de l'île $C_{\Sigma} \equiv$ $C_{J 1}+C_{J 2}+C_{g}+2 C_{J S}$. Dans le but de simplifier la situation, on suppose que les jonctions formant le SQUID sont identiques $C_{J S 1}=C_{J S 2} \equiv C_{J S}, E_{J S 1}=E_{J S 2} \equiv E_{J S}$.

Afin de connaitre l'efficacité de ce détecteur, on cherche premièrement à déterminer le supercourant dans le SSET. Ce dernier peut être obtenu à l'aide de la relation usuelle

$$
I_{s}=\frac{2 e}{\hbar} \frac{\partial E_{f}}{\partial \theta}
$$

où $E_{f}$ est l'énergie du fondamental de (5.11). Dans la situation où $E_{C}>E_{J}$, il est possible de ne considérer que deux états de charge pour l'île du SSET. Dans ce cas, le remplacement (3.23) vers les matrices de Pauli peut être fait et $E_{f}$ s'obtient simplement.

Dans le cas où $E_{J} \gtrsim E_{C}$ cependant, cette approximation ne suffit plus. On doit alors diagonaliser numériquement l'hamiltonien (5.11) en utilisant plusieurs états de charge. De façon alternative, on peut faire le passage vers la représentation de phase $n \rightarrow-i \partial / \partial \phi$. L'hamiltonien (5.11) se reécrit alors sous la forme de l'équation différentielle de Mathieu dont les solutions sont des superpositions des fonctions de Bloch $[208,217]$. Des résultats obtenus à l'aide de la première approche seront brièvement présentés à la fin de cette section mais seront présentés en plus de détail ailleurs. Ici, on présente des résultats basés sur l'approximation à deux états de charges. Cette approche permet d'obtenir des résultats qualitatifs sur le fonctionnement de ce détecteur.

On commence par réécrire l'énergie potentielle de (5.11) sous la forme

$$
\begin{aligned}
U= & -\left\{\left(E_{J 1}+E_{J 2}\right) \cos \theta / 2+2 E_{J S} \cos \frac{\pi}{\Phi_{0}}\left(\Phi_{x}+\Phi_{q}\right) \cos \theta_{\text {res }}\right\} \cos \phi \\
& -\left\{\left(E_{J 1}-E_{J 2}\right) \sin \theta / 2+2 E_{J S} \cos \frac{\pi}{\Phi_{0}}\left(\Phi_{x}+\Phi_{q}\right) \sin \theta_{\text {res }}\right\} \sin \phi \\
\equiv & -A \cos \phi-B \sin \phi .
\end{aligned}
$$

Le remplacement (3.23) donne alors pour (5.11)

$$
H_{2}=\frac{E_{C}}{2}\left(1-2 n_{g}\right) \tau_{z}-\frac{A}{2} \tau_{x}-\frac{B}{2} \tau_{y}
$$


où les $\tau_{i}$ sont les matrices de Pauli pour le SSET. Dans cette approximation, le fondamental est alors simplement

$$
E_{f}=-\frac{1}{2} \sqrt{E_{C}^{2}\left(1-2 n_{g}\right)^{2}+A^{2}+B^{2}} .
$$

Afin de déterminer le supercourant, on commence par minimiser $E_{f}$ par rapport à la phase du réservoir. Cette minimisation tient compte du fait qu'il n'y a pas de courant continu dans le SQUID (en raison de la capacité $C_{r e s}$ ):

$$
\left.\frac{\partial E_{f}}{\partial \theta_{\text {res }}}\right|_{\theta_{\text {res }}^{0}}=0 \Rightarrow \theta_{r e s}^{0}=\tan ^{-1}\left(\frac{E_{J 1}-E_{J 2}}{E_{J 1}+E_{J 2}} \tan \frac{\theta}{2}\right) \text {. }
$$

Lorsque les jonctions du SSET ont la même énergie Josephson, $E_{J 1}=E_{J 2} \equiv E_{J}$, on obtient $\theta_{r e s}^{0}=0$. Afin de simplifier les calculs, on s'intéressera à cette situation par la suite.

En remplaçant ces résultats dans $E_{f}$, on obtient facilement de (5.12) l'expression pour le supercourant :

$$
I_{s}=\frac{I_{0}}{4} \frac{\sin \theta+2 \tilde{E}_{J S} \cos \frac{\pi}{\Phi_{0}}\left(\Phi_{x}+\Phi_{q}\right) \sin \theta / 2}{\sqrt{\left[\frac{\tilde{E}_{C}}{2}\left(1-2 n_{g}\right)\right]^{2}+\left[\cos \theta / 2+\tilde{E}_{J S} \cos \frac{\pi}{\Phi_{0}}\left(\Phi_{x}+\Phi_{q}\right)\right]^{2}}}
$$

où $I_{0} \equiv 2 e E_{J} / \hbar, \tilde{E}_{J S} \equiv E_{J S} / E_{J}$ et $\tilde{E}_{C} \equiv E_{C} / E_{J}$. Si le réservoir de phase est déconnecté de l'île, c'est-à-dire pour $\Phi_{x}+\Phi_{q}=\Phi_{0} / 2$, on retrouve la relation usuelle pour le supercourant d'un SSET [122,123].

Le courant critique est obtenu numériquement en maximisant $I_{s}$ par rapport à $\theta$ : $I_{c}\left(n_{g}, \Phi_{x}, \Phi_{q}\right)=\operatorname{Max}_{\theta}\left\{I_{s}\left(\theta, n_{g}, \Phi_{x}, \Phi_{q}\right)\right\}$. La figure 5.7 a) présente le courant critique en fonction des paramètres de contrôle $n_{g}$ et $\Phi_{x}$. Les énergies du système sont prises comme dans l'expérience décrite à la section précédente : $\tilde{E}_{J S}=0.84$ et $\tilde{E}_{C}=2.48$. Le flux dans le SQUID dû au qubit est $\Phi_{q}=10^{-3} \Phi_{0}$ [135]. Comme il se doit, le courant critique est périodique en $\Phi_{0}$ et est symétrique par rapport à $n_{g}=1 / 2$. On reproduit donc qualitativement les résultats expérimentaux de Matters et al. [222].

La figure $5.7 \mathrm{~b}$ ) présente la différence de courant critique $\Delta I_{c}=I_{c+}-I_{c-}$ correspondant au flux des états logiques du qubit $\Phi_{q}= \pm 10^{-3} \Phi_{0}$. Dans certaines gammes de paramètres $\left\{n_{g}, \Phi_{x}\right\}$, la différence $\Delta I_{c}$ est non-nulle tandis qu'elle s'annule pour certains $\left\{n_{g}, \Phi_{x}\right\}$. Ces dernières régions sont mises en relief par de larges lignes noires 


\subsection{LECTURE DU QUBIT DE FLUX}

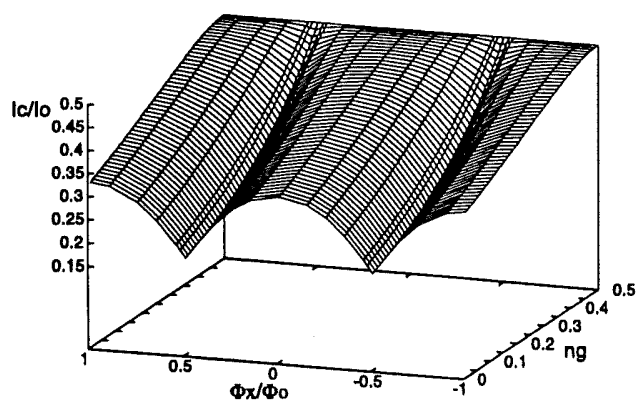

(a)

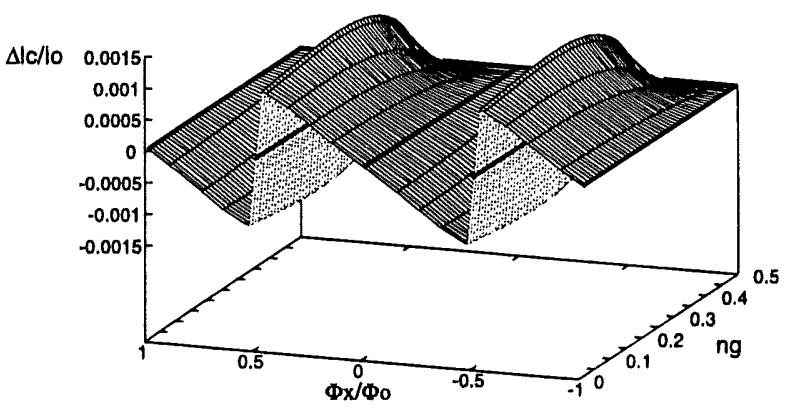

(b)

FIG. 5.7: a) Courant critique en fonction du voltage de grille et du flux dans le SQUID. b) Différence de courant critique lorsque le qubit contribue $\Phi_{q}= \pm 10^{-3} \Phi_{0}$ au flux dans le SQUID. Les régions où cette différence s'annule sont mises en évidence par de larges lignes noires. Les énergies sont choisies comme à la figure $5.5: \tilde{E}_{J S}=0.84$ et $\tilde{E}_{C}=2.48$.

à la figure $5.7 \mathrm{~b}$ ). Lorsque $\Delta I_{c} \neq 0$, le détecteur est dans l'état 'ouvert'. À l'opposé, le détecteur est dans l'état 'fermé' lorsque $\Delta I_{c}=0$. Lors des manipulations cohérentes, on garde le détecteur dans l'état 'fermé'. Pour la lecture, on place ce dernier dans l'état 'ouvert' et on applique un courant dans le SSET. Le voltage aux bornes du SSET est le résultat de la mesure et contient l'information sur l'état du qubit. Ceci est discuté plus amplement à la section $§ 5.4 .4$.

Même sans appliquer de courant (ou pour un courant inférieur à $I_{c}$ ), lorsque le détecteur est dans l'état 'ouvert' celui-ci présente des valeurs différentes de $I_{c}$ à l'environnement. L'environnement peut alors 'mesurer' le qubit, ce qui accélère la perte de cohérence. On ne considère ici que la partie de l'environnement due à l'impédance du détecteur. Un aspect intéressant de cette approche est que, lorsque le détecteur est dans l'état fermé, le système présente le même courant critique à l'environnement. Celui-ci ne peut alors distinguer les états du qubit, ce qui conduit à des taux de décohérence plus faibles.

On remarque de la figure $5.7 \mathrm{~b})$ que le choix des paramètres $\left(n_{g}, \Phi_{x}\right)$ pour l'état fermé du détecteur est important. En effet, les choix $\left(n_{g}= \pm 1 / 2, \Phi_{x}=0, \pm 1 / 2\right)$ semblent particulièrement intéressants puisqu'alors $\Delta I_{c}$ est insensible aux fluctuations de $n_{g}$ et $\Phi_{x}$. Toutefois, ce type de points spéciaux est généralement sensible à 
l'asymétrie du système. Par exemple, dans le cas du INSQUID [214], le SQUID-dc de lecture ne sera déconnecté du qubit que si les jonctions du INSQUID sont identiques. De même, pour le qubit phase-charge de Saclay la symétrie des jonctions formant l'île du qubit est importante [126]. Si ces jonctions ne sont pas identiques, un courant circulera dans la boucle du qubit même au point d'opération. Ceci diminue le temps de cohérence du système. Pour le détecteur qui nous intéresse ici, il apparaît donc important de vérifier la dépendance de ces performances à l'asymétrie.

De plus, l'approche à deux niveaux présentée jusqu'à présent ne s'applique qu'au cas où l'énergie de charge domine. Cette approche a toutefois l'avantage de fournir facilement des résultats analytiques donnant un aperçu de la physique importante du système. Pour obtenir des résultats quantitatifs et s'appliquant à une plus grande gamme de valeurs du ratio $E_{C} / E_{J}$, l'approximation à deux niveaux ne suffit plus. Afin d'aller au-delà de cette approximation, on diagonalise numériquement l'hamiltonien du circuit de détection (5.11). Afin de tenir compte de certaines contraintes de fabrication, on ne supposera pas que les jonctions du SSET ou du SQUID sont identiques. Dans ce but, on remplace le dernier terme de l'hamiltonien (5.11) qui n'est valide que pour un SQUID symétrique par $-E_{J}\left(\Phi_{x}\right) \cos \left(\phi-\theta_{\text {res }}-\alpha\right)$, où

$$
E_{J}\left(\Phi_{x}\right)=\sqrt{\left(E_{J S 1}-E_{J S 2}\right)^{2}+4 E_{J S 1} E_{J S 2} \cos ^{2}\left(\frac{\pi}{\Phi_{0}}\left(\Phi_{x}+\Phi_{q}\right)\right)}
$$

et

$$
\tan \alpha=\frac{E_{J S 1}-E_{J S 2}}{E_{J S 1}+E_{J S 2}} \tan \left(\frac{\pi}{\Phi_{0}}\left(\Phi_{x}+\Phi_{q}\right)\right),
$$

tel que décrit à l'annexe $\mathrm{A}$.

La figure 5.8 présente le courant critique et la différence de courant critique obtenus à partir de la diagonalisation exacte et pour les mêmes valeurs de paramètres qu'à la figure 5.7. Dans ce cas, il n'y a donc pas d'asymétrie dans le système. On remarque premièrement de la figure 5.8 a) que la suppression du courant critique n'est pas complètement levée à la résonance, contrairement au résultat obtenu de l'approximation à deux niveaux. De plus, il en découle qu'au point d'opération $\left(n_{g}= \pm 1 / 2, \Phi_{x}=0, \pm 1 / 2\right)$, la pente de $\Delta I_{c}$ ne s'annule plus dans la direction $n_{g}$. Par conséquent, le système devient plus sensible aux fluctuations du voltage. En travaillant avec une plus grande énergie Josephson $\tilde{E}_{J S}$ pour le SQUID, comme aux 


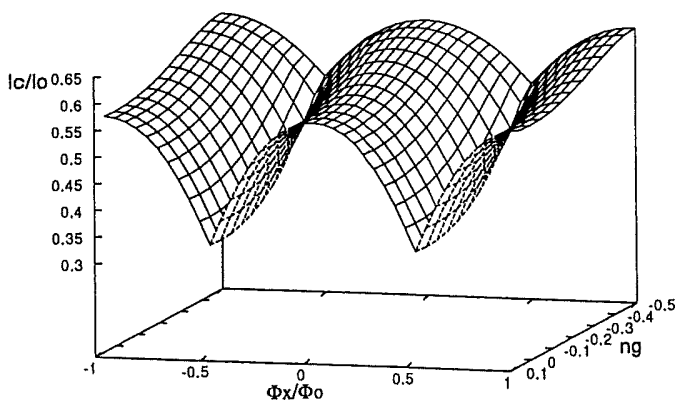

(a)

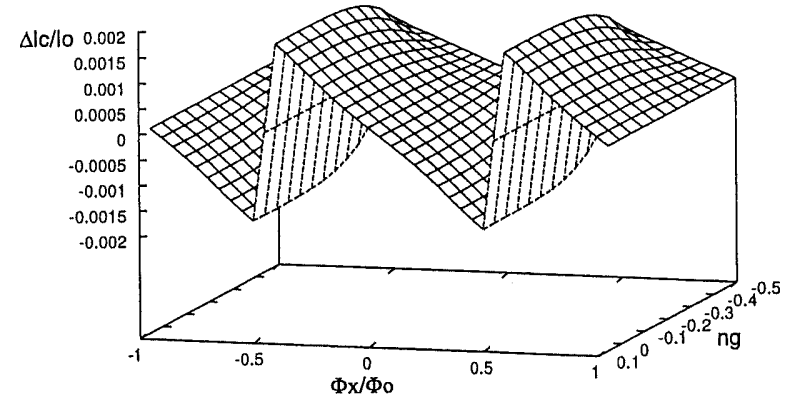

(b)

FIG. 5.8: a) Courant critique obtenu par diagonalisation exacte en fonction du voltage de grille et du flux dans le SQUID. b) Différence de courant critique lorsque le qubit contribue $\Phi_{q}= \pm 10^{-3} \Phi_{0}$ au flux dans le SQUID. Les énergies sont les mêmes qu'aux figures 5.5 et $5.7: \tilde{E}_{J S}=0.84$ et $\tilde{E}_{C}=2.48$.

figures $5.9 \mathrm{a}$ ) et b), on réussit toutefois à retrouver une certaine insensibilité aux fluctuations de voltage. Dans le cas illustré aux figures 5.9 a) et b), l'énergie de charge aussi est importante $\left(\tilde{E}_{C}=10, \tilde{E}_{J S}=10\right)$ de sorte que le courant critique est fortement supprimé hors de la résonance. De plus, puisque $\tilde{E}_{J S}$ est grand, le couplage au réservoir de phase est important. De ce fait, la suppression du courant critique est rapidement levée lorsque le flux externe s'éloigne de $\Phi_{x} / \Phi_{0}= \pm 1 / 2$. Hors de ces valeurs particulières du flux externe, le courant critique sature rapidement et $I_{c}$ présente un plateau autour de $\Phi_{x} / \Phi_{0}=0$. Comme on le voit à la figure $5.9 \mathrm{~b}$ ), cette combinaison de la suppression importante du courant critique par l'énergie de charge et d'un couplage de grande intensité entre le réservoir de phase et l'île du SSET produit un $\Delta I_{c}$ ayant des pics de grande amplitude. Dans une telle situation, il est plus facile de distinguer les états logiques du qubit. Cette plus grande amplitude a toutefois l'inconvénient important que les pics de $\Delta I_{c}$ sont très étroits et donc que les fluctuations de voltage et de flux deviennent importantes lors de la lecture. Il est important d'optimiser les paramètres du système de façon à atteindre le juste milieu entre grande amplitude et faible largeur des pics de $\Delta I_{c}$. Notons qu'en pratique on ne peut choisir l'énergie de charge arbitrairement grande puisque, tel que mentionné à la section 3.2.1, le gap supraconducteur doit dominer toutes les autres énergies du 


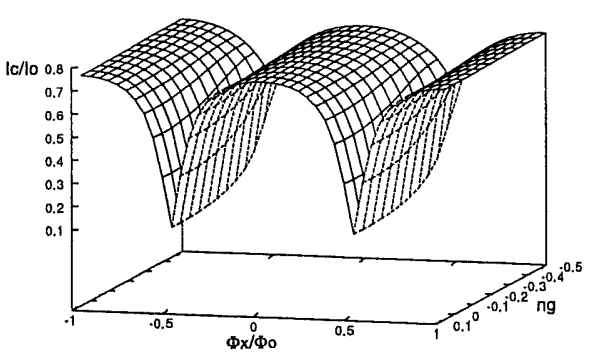

(a)

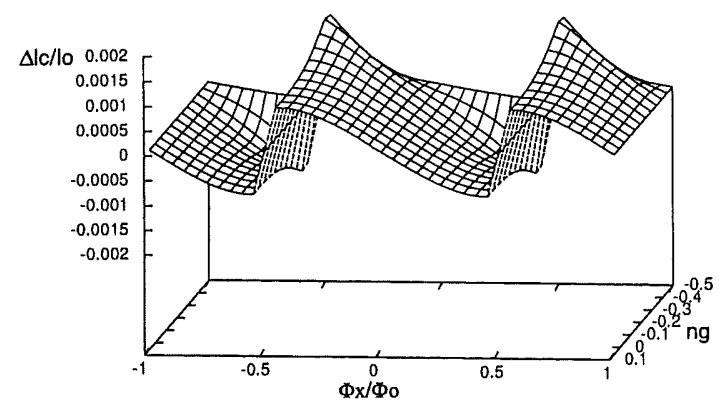

(c)

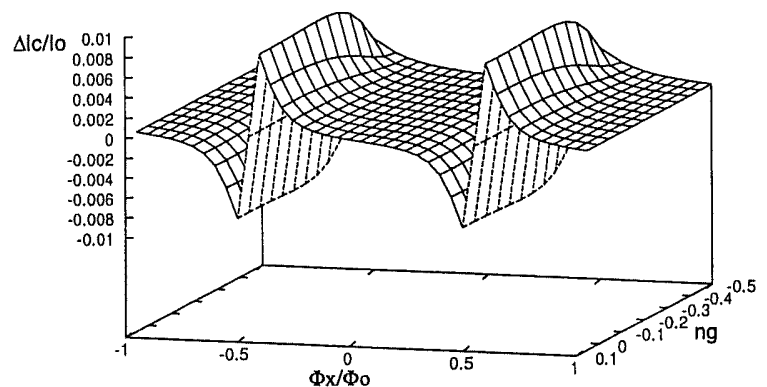

(b)

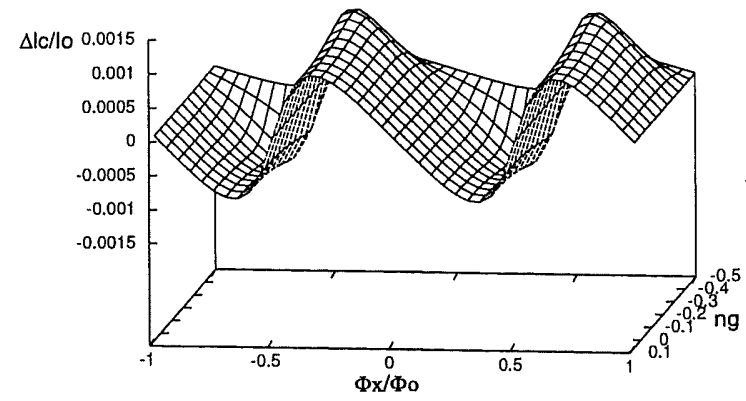

(d)

FIG. 5.9: Courant critique (a) et différence de courant critique (b,c,d) en fonction du voltage de grille et du flux externe. En a) et b), les énergies sont $\tilde{E}_{J S}=10$ et $\tilde{E}_{C}=10$. De même, les jonctions du SSET et du SQUID sont symétriques. En c) et d), l'énergie de charge est $\tilde{E}_{C}=10$ et les jonctions du SSET sont symétriques. En c) on prend $\tilde{E}_{J S 1}=\tilde{E}_{J S 2}=1$ tandis qu'en d) on prend plutôt $\tilde{E}_{J S 1}=1$ et $\tilde{E}_{J S 2}=2$. 


\subsection{LECTURE DU QUBIT DE FLUX}

système. Dans le cas contraire, l'effet tunnel de quasiparticules sur l'île du SSET n'est pas supprimé.

Tel que mentionné précédemment, il est important d'étudier l'effet de l'asymétrie sur $\Delta I_{C}$. Premièrement, les calculs numériques par diagonalisation exacte montrent que lorsque les jonctions du SSET ne sont pas équivalentes $\left(E_{J 1} \neq E_{J 2}\right)$, l'amplitude de $\Delta I_{C}$ diminue. La raison en est simple : avec deux jonctions en série, l'énergie Josephson de la plus faible dicte l'énergie Josephson effective de la paire. Une légère asymétrie n'est donc pas un problème en pratique pour ce détecteur (elle ne change pas la structure de $\Delta I_{c}$ en fonction du voltage et du flux, seulement son amplitude). En second lieu, on s'intéresse à l'asymétrie des jonctions du SQUID $\left(E_{J S 1} \neq E_{J S 2}\right)$. Dans le cas où une des jonctions domine (i.e. $E_{J S 1} \gg E_{J S 2}$ ), il vient de (5.18) que le SQUID agit comme une simple jonction et dont l'énergie Josephson s'approche de l'énergie de la jonction qui domine. Dans ce cas, on vérifie que le flux externe n'a que peu d'influence sur le courant critique et que le réservoir de phase est effectivement toujours couplé à l'île du SSET. Dans un cas moins extrême où une jonction ne domine pas complètement, l'asymétrie du SQUID peut être avantageuse. Les figures $5.9 \mathrm{c}$ ) et d) présentent la différence de courant critique dans le cas où $\tilde{E}_{C}=10, E_{J 2} / E_{J 1}=1$ et, respectivement, $\tilde{E}_{J S 2}=\tilde{E}_{J S 1}$ et $\tilde{E}_{J S 2}=\tilde{E}_{J S 1} / 2$. On remarque que dans le second cas, les maximums de $\Delta I_{c}$ sont arrondis. On peut alors trouver un point d'opération pour la lecture où les fluctuations de voltages s'annulent au premier ordre. Ceci est évidemment avantageux.

\subsubsection{Sensibilité et rétroaction}

Afin de déterminer l'effet de l'environnement sur le qubit avant la mesure $\left(I<I_{c}\right)$ on peut suivre l'approche de Tian et al. [135]. Dans cette approche, on commence par linéariser l'hamiltonien du circuit de détection. On identifie ensuite chaque terme de l'hamiltonien linéarisé à une composante d'un circuit linéaire (capacité et inductance). On obtient alors un circuit équivalent pour lequel il est facile de déterminer l'impédance effective telle que vue par le qubit. De ce résultat, et comme aux chapitres 3 et 4 , on obtient finalement les taux de relaxation et de déphasage.

Afin d'appliquer cette approche au circuit qui nous intéresse ici, on doit d'abord 
remarquer qu'il n'est pas possible de linéariser par rapport à la variable interne $\phi$ du SSET dans le régime $E_{C}>E_{J}$. Cette linéarisation ne tient pas compte du blocage de Coulomb qui est important pour la dynamique de $\phi$. En supposant que le SSET reste dans son fondamental, il semble alors plus approprié de faire cette linéarisation en ayant d'abord remplacé, comme précédemment, dans l'hamiltonien total les termes décrivant le SSET par l'énergie de son fondamental. Cette approche ne nous renseigne toutefois que sur le régime $I<I_{c}$. Afin de déterminer la sensibilité de ce détecteur au flux du qubit, on s'intéresse ici plutôt au régime $I>I_{c}$.

En considérant à nouveau le cas $E_{J 1}=E_{J 2}$, on obtient de (5.13) l'hamiltonien d'interaction qubit-détecteur suivant :

$$
H_{\text {int }}=2 E_{J S} \sin \frac{\pi \Phi_{x}}{\Phi_{0}} \sin \frac{\pi \Phi_{q}}{\Phi_{0}} \cos \phi
$$

Avec $\eta \approx 2 \pi\left(\left|\Phi_{q}\right| / \Phi_{0}\right) E_{J S} \sin \left(\pi \Phi_{x} / \Phi_{0}\right)$ pour $\left|\Phi_{q}\right| / \Phi_{0} \ll 1$, on retrouve la forme standard $H_{\text {int }}=\eta \sigma_{z} \cos \phi$ où la phase $\phi$ joue le rôle du pointeur. Afin d'obtenir les caractéristiques du détecteur, on peut alors utiliser l'approche décrite à la section $\S 5.2$.

On commence ici par déterminer le temps de lecture. Un calcul semblable a été réalisé pour la sensibilité du SSET à la charge par Zorin [217]. On suit ici les mêmes approche et notation.

On fixe $\Phi_{q}=0$ et on s'intéresse à la réponse du détecteur à une variation du flux $\Phi_{x}$. La sensibilité du détecteur au flux est donnée par

$$
\delta \Phi=\frac{V_{f}}{\lambda} \quad[\text { Flux } / \sqrt{\mathrm{Hz}}] .
$$

Dans cette expression, $\lambda$ est la fonction de réponse

$$
\lambda=\frac{\partial V}{\partial \Phi_{x}}
$$

et $V_{f}$ l'amplitude des fluctuations de voltage aux bornes du SSET

$$
V_{f}=\sqrt{2 \pi S_{V}(0)} \cdot[\mathrm{V} / \sqrt{\mathrm{Hz}}] .
$$

La signification de la relation (5.21) est que, étant donnée une sensibilité $\delta \Phi$, une variation de flux de $\delta \Phi$ dans le SQUID pourra être détectée dans un temps de mesure d'une seconde [206]. 
Le SSET est équivalent à une jonction Josephson dans le régime sur-amorti et de courant critique $I_{c}\left(n_{g}, \Phi_{x}\right)$. Pour les fluctuations de voltage à ces bornes, on peut alors utiliser les résultats du modèle RSJ [208,217, 225-227] :

$$
S_{V}(0)=\frac{2 R_{d}^{2}}{\pi R_{s}}\left(k_{B} T+\frac{I_{c}^{2}}{2 I} e V \operatorname{coth} \frac{e V}{k_{B} T}\right) .
$$

Dans cette expression, $R_{d}=\partial V / \partial I$ est la résistance différentielle de la jonction effective. Toujours dans le modèle RSJ sur-amorti, le voltage moyen $V$ entrant dans l'expression pour $S_{V}(0)$ s'obtient de l'équation du mouvement pour la phase

$$
\frac{\Phi_{0}}{2 \pi R_{s}} \dot{\theta}+I_{s}\left(\theta, n_{g}, \Phi_{x}\right)=0
$$

en intégrant $\dot{\theta}$ sur une période du supercourant. Pour une jonction ayant une relation courant-phase purement sinusoïdale, on obtient la relation hyperbolique usuelle $V / I_{c} R_{s}=\left(I / I_{c}-1\right)^{1 / 2}$, pour $I / I_{c}>1$ [208]. En utilisant plutôt la relation courantphase (5.17), on observe après intégration numérique de (5.25) que le voltage s'approche de cette forme.

De la sensibilité $\delta \Phi$, on obtient facilement le temps de mesure. Pour la mesure d'un qubit, on cherche à distinguer entre deux valeurs de flux $\Phi_{q}$ séparées de $\Delta \Phi=$ $2\left|\Phi_{q}\right|=2 \cdot 10^{-3} \Phi_{0}$. Le temps de mesure $t_{m e s}$ est donc

$$
\Delta \Phi=\frac{\delta \Phi}{\sqrt{t_{m e s}}} \Rightarrow t_{m e s}=\left(\frac{\delta \Phi}{\Delta \Phi}\right)^{2}=\frac{2 \pi S_{V}(0)}{\lambda^{2} \Delta \Phi^{2}}
$$

Numériquement, on obtient facilement ce temps après avoir calculé le courant critique et le voltage moyen. Des commentaires sur ce calcul seront donnés plus loin.

On s'intéresse maintenant à la rétroaction du détecteur sur le qubit et, plus particulièrement, au mélange des états du qubit qui en résulte. On aborde ce problème de façon légèrement différente de la section §5.2. On s'intéresse ici à l'effet de l'environnement $R_{s}$ du détecteur sur les niveaux d'énergie du qubit. En effet, $R_{s}$ induit des fluctuations de voltage $V_{f}$ aux bornes du SSET. Celles-ci sont responsables de fluctuations de voltage de l'île du SSET qui se traduisent en fluctuations de voltage aux bornes du SQUID. Ces dernières induisent finalement des fluctuations de courant qui se couplent au qubit. 
Le voltage $U_{\phi}$ de l'île du SSET est donné par

$$
U_{\phi}=\frac{1}{2 e} \frac{\partial E_{f}}{\partial n_{g}} .
$$

Dans l'approximation à deux états de charge pour le SSET, on utilise à nouveau la relation (5.15) pour le fondamental $E_{f}$.

Pour de petites fluctuations, les fluctuations de $U_{\phi}$ sont reliées à celles de la phase externe $\theta$ du SSET $\delta U=(\partial U / \partial \theta) \delta \theta$. De même, $\theta$ est relié à $V_{f}$ par la relation de Josephson $V_{f}=i \omega \Phi_{0} \theta / 2 \pi$. On obtient alors

$$
\left\langle U_{\phi} U_{\phi}\right\rangle_{\omega}=\frac{1}{\omega^{2}}\left(\frac{2 \pi}{\Phi_{0}}\right)^{2}\left(\frac{\partial U}{\partial \theta}\right)^{2}\left\langle V_{f} V_{f}\right\rangle_{\omega}
$$

Pour $\left\langle V_{f} V_{f}\right\rangle_{\omega}$, on ne peut utiliser la relation (5.24) qui n'est valide qu'à basse fréquence. Puisque l'on cherche à déterminer la relaxation induite par le détecteur, on doit prendre une relation valide à la fréquence de transition $\omega_{0}$ du qubit. On utilise donc ici la relation obtenue dans le modèle RSJ et dont (5.24) est la limite basse fréquence $[208,225-227]$ :

$$
S_{V}(\omega)=\frac{2 e I_{c} R_{s}}{\Phi_{0}} \sum_{k=-\infty}^{\infty}\left|z_{k}\right|^{2}(\theta-k \nu) \operatorname{coth}\left[\frac{e I_{c} R_{s}}{k_{B} T}(\theta-k \nu)\right]
$$

où

$$
\left|z_{k}\right|=\left|\delta_{k, 0}+\frac{k i(i-\nu)^{|k|}}{\theta-k \nu}-\frac{1}{2}\left[\frac{(k-1)(i-\nu)^{|k-1|}}{\theta-(k-1) \nu}+\frac{(k+1)(i-\nu)^{|k+1|}}{\theta-(k+1) \nu}\right]\right| .
$$

Dans ces expressions, $\nu=V / I_{c} R_{s}, i \equiv I / I_{c}$ et $\theta \equiv \omega / \omega_{J}$ où $\omega_{J}=2 \pi I_{c} R_{s} / \Phi_{0}$. La densité spectrale $S_{V}(\omega)$ a plusieurs pics [225]. Le taux de relaxation sera minimisé si aucun de ceux-ci ne se trouve à $\omega_{0}$. On remplace finalement $\left\langle V_{f} V_{f}\right\rangle_{\omega}$ dans (5.28) par $S_{V}(\omega)$ afin d'obtenir une expression pour $\left\langle U_{\phi} U_{\phi}\right\rangle_{\omega}$.

Avec ce résultat, il est maintenant simple de déterminer l'effet de ces fluctuations sur le qubit. La capacité du réservoir à la masse est grande et on considèrera le réservoir comme étant effectivement à la masse. Le degré de liberté interne $\phi \mathrm{du}$ SSET correspond au degré de liberté externe du SQUID et les fluctuations de $U_{\phi}$ sont des fluctuations de voltages aux bornes du SQUID. Pour déterminer leur effet sur l'asymétrie $\epsilon=2 I_{s}\left(\Phi_{x}-\Phi_{0} / 2\right)$ du double puits de potentiel (voir section §3.2.2), 
on peut alors utiliser directement les résultats des références $[125,136]$. Dans ces références, les auteurs s'intéressent à l'effet sur un qubit de flux à trois jonctions de la présence d'un SQUID-dc. On obtient alors

$$
\langle\epsilon \epsilon\rangle_{\omega}=8 I_{c s}^{2} \frac{(2 \pi)^{2}}{\omega^{4}}\left(\frac{2 \pi}{\Phi_{0}}\right)^{2}\left(\frac{\Phi_{q}}{\Phi_{0}}\right)^{2}\left(\frac{\partial U}{\partial \theta}\right)^{2} \sin ^{2} \phi \sin ^{2}\left(\frac{\pi \Phi_{x}}{\Phi_{0}}\right) S_{V}(\omega),
$$

où $I_{c s}=2 e E_{J S} / \hbar$ est le courant critique du SQUID. On remarque qu'à $\Phi_{x}^{\prime}=0$, les fluctuations sont découplées du qubit. C'est le résultat auquel on était en droit de s'attendre à partir de la forme, présentée à la figure 5.7, que prend la différence de courant critique en fonction du flux externe. Dans cette dernière expression, on fait le remplacement

$$
\frac{\partial U}{\partial \theta} \sin \phi \quad \rightarrow \quad \operatorname{Max}\left\{\frac{\partial U}{\partial \theta}\right\}
$$

dans le but de considérer le pire des cas. On obtient finalement la densité spectrale $J(\omega)=\langle\epsilon \epsilon\rangle_{\omega} / \hbar^{2}$ et le temps de mélange de (3.31). Numériquement, on obtient facilement ce taux après avoir déterminé le courant critique et le voltage moyen.

Ayant obtenu ces relations, on cherche numériquement une région de l'espace des paramètres pour laquelle le temps de mesure est inférieur au temps de mélange. Une étude préliminaire tend à montrer que le ratio $t_{m e s} / t_{m i x}$ sera minimisé pour $E_{c} / E_{J}<$ 1. Notons que le grand nombre de paramètres impliqués ne facilite pas l'optimisation numérique des performances du détecteur. Pour faciliter cette optimisation, il pourrait être utile de recourir aux techniques du type recuit simulé, déjà abordées au chapitre 1 .

\subsubsection{Remarques et sommaire}

Quelques remarques sont de mise.

(1) Il y a (au moins) deux façons d'utiliser ce détecteur. Premièrement, en appliquant un courant dans le SSET qui est supérieur à $I_{c+}$ et $I_{c-}$. On mesure alors le voltage qui sera différent selon l'état du qubit. La deuxième approche est d'appliquer un courant entre les valeurs $I_{c+}$ et $I_{c-}$. On mesurera alors un voltage ou aucun voltage selon la valeur logique du qubit.

(2) Le voltage de sortie aux bornes du SSET sera fort probablement très petit. En effet, afin que le SSET soit dans le régime sur-amoti, on doit avoir $R_{s} \sim 100 \Omega$. De 


\subsection{LECTURE DU QUBIT DE FLUX}

plus, les performances du SSET semblent être optimisées lorsque le courant critique $I_{0}$ n'est pas trop élevé, de l'ordre de $10-100 n A$ [141]. Le voltage de sortie sera donc au mieux de l'ordre $\sim 10^{-5}, 10^{-6} \mathrm{~V}$. Dans le mode d'opération où l'on applique un courant au SSET supérieur au courant critique correspondant à chacun des états du qubit, la différence de voltage entre ces deux états sera d'environ $10^{-2}$ à $10^{-3}$ fois plus faible que le voltage de sortie. Tel que mentionné à la section $§ 5.3 .2$, il sera alors nécessaire d'utiliser un amplificateur à basse température. Celui-ci injecte du bruit dans le système augmentant ainsi le temps de mesure requis pour distinguer les états du qubit.

(3) Il faut tenir compte des contributions des fluctuations de $n_{g}$ et de $\Phi_{x}$ à $U_{\phi}$.

(4) Il serait intéressant d'appliquer l'approche décrite aux références [177, 228, 229] et mentionnée en annexe $\mathrm{D}$ pour modifier la valeur $R_{s}$ selon que la mesure est en cours ou non. Dans cette approche, $R_{s}$ est remplacée par un réseau unidimensionnel de SQUIDs. On change alors la résistance effective en ajustant un flux extérieur sur le réseau. Avec cette technique, Watanabe et Haviland [228] ont pu moduler, sur plusieurs ordres de grandeur, la résistance effective vue par une jonction Josephson et ainsi étudier la dynamique d'une même jonction en présence de différents environnements. Notons que cette approche pourrait aussi être utilisée pour améliorer le comportement du SQUID-dc lors de la lecture d'un qubit.

Le détecteur pour qubits de phase suggéré ici semble avoir, tout au moins pour certaines valeurs de ces paramètres, l'avantage important de permettre la séparation entre les parties dissipative et cohérente du circuit. En choisissant le point d'opération du détecteur, on peut optimiser la mesure ou le temps de cohérence du qubit. Un problème potentiel toutefois réside dans le fait que le signal de sortie est très petit. On doit alors utiliser un étage d'amplification qui réintroduit du bruit dans le système. Il s'agit d'un problème du SSET et il est cependant peut-être possible d'appliquer le concept de transducteur phase-charge étudié ici à un détecteur ne présentant pas ce problème. Finalement, avant de conclure sur l'efficacité du détecteur présenté ici, il est nécessaire de compléter l'optimisation des performances sur toute la gamme de valeurs des paramètres du système. 


\section{Conclusion}

Where a calculator on the ENIAC is equipped with 18000 vacuum tubes and weighs 30 tons, computers in the future may have only 1000 vacuum tubes and perhaps weigh one and a half ton.

-Popular Mechanics, 1949

Dans cette thèse, on s'est intéressé à différents aspects du calcul quantique et, plus particulièrement, aux qubits supraconducteurs. On a d'abord considéré la réalisation des opérations logiques et vu comment il peut parfois être avantageux d'utiliser des codes pour arriver à l'universalité. Pour les qubits supraconducteurs de phase, l'utilisation de ce type de code a permis la simplification des designs (annexe C) [52].

De plus, on a vu comment un ordinateur quantique pourrait être utile à des problèmes d'intérêt physique, comme l'estimation de valeurs propres. Ces algorithmes quantiques requièrent un grand nombre d'opérations logiques et il est avantageux d'optimiser leur application. En particulier, il est important que cette optimisation prenne en considération la topologie du registre de qubits. Pour la transformée de Fourier exacte et un registre unidimensionnel de qubits limités à des interactions aux plus proches voisins, nous avons obtenus des circuits de profondeur linéaire [67]. Dans le contexte du design de registres quantiques, ce résultat met en évidence l'importance de la possibilité de réaliser certaines opérations logiques en parallèle.

L'application de la phase géométrique non-adiabatique au calcul quantique a ensuite été étudiée [99]. Cette phase a plusieurs avantages par rapport à la phase de Berry. (1) Elle peut être réalisée plus rapidement. (2) Avec un choix approprié du parcours dans l'espace projectif, elle ne nécessite pas d'annulation de la phase dynamique. Finalement, (3) seulement deux champs de contrôle sont requis. Cette dernière simpli- 
fication peut faciliter les manipulations et, dans certains cas, la fabrication des qubits. Pour le calcul quantique, l'intérêt des phases géométriques est leur supposée tolérance aux imperfections. Malheureusement, on a montré que les phases géométriques sont en fait moins tolérantes aux imperfections que leurs équivalents dynamiques. Ce résultat est en accord avec l'approche complètement numérique de Nazir et al. [110]. Malgré cela, il serait intéressant d'observer cette phase à l'aide de systèmes supraconducteurs mésoscopiques. On a montré comment cela est possible pour les qubits de charge. L'application à d'autres systèmes n'est qu'une simple généralisation.

Après s'être intéressé à ces questions plus algorithmiques, différents aspects concernant les qubits supraconducteurs ont été abordés. On a, entre autres, présenté un nouveau type de qubit basé sur une combinaison de supraconducteurs de type $s$ et de type $d$ (annexe D) [162]. De plus, on a étudié l'interaction capacitive entre une paire de qubits basés sur les jonctions Josephson dans lesquels un courant est appliqué (current-biased Josephson junction, CBJJ) [179]. L'effet de la fuite hors des états logiques des jonctions et des fluctuations de courant a été analysé. On a ensuite montré comment une CBJJ peut être utile pour faire interagir indirectement des qubits. Ce type d'interaction a l'avantage d'être ajustable. De plus, à l'aide du 'recoupling' cette méthode peut être appliqué à différents types de qubits. Une confirmation expérimentale de ces résultats serait intéressante.

Finalement, un transducteur phase-charge pour la lecture du qubit de flux a été suggéré. Un avantage de ce détecteur est l'existence d'un point d'opération où l'environnement est découplé du qubit. Il est nécessaire de compléter cette étude en allant au-delà de l'approximation à deux charges pour le SSET et en optimisant les performances du détecteur par une recherche dans l'espace de ces paramètres.

En résumé, on a étudié dans ce document plusieurs aspects concernant les ordinateurs quantiques et leur réalisation à l'aide de systèmes supraconducteurs. À travers ces différents aspects, il apparaît clairement que l'informatique quantique est un champ de recherche se situant à la frontière entre de nombreuses spécialités. Une connaissance de ces différentes spécialités est nécessaire afin de pouvoir faire le parallèle entre les exigences algorithmiques et les limitations physiques et pratiques des dispositifs. Par exemple, on a vu que l'utilisation des codes pouvait réduire certaines contraintes de fabrication des dispositifs. À l'opposé, on a vu que les codes correcteurs 
d'erreurs imposent des conditions sévères sur les appareils de mesure et qu'on doit en tenir compte dans leur design.

Dans ce document, quatre suggestions expérimentales ont été faites. (1) Observation de la phase géométrique non-adiabatique dans les systèmes supraconducteurs mésoscopiques. (2) Suggestion d'un nouveau design de qubit supraconducteur. (3) Méthode pour l'interaction qubit-qubit. (4) Nouvelle approche pour la lecture d'un qubit de flux. À l'exception du nouveau design de qubit qui semble plus difficile à fabriquer, les trois autres suggestions apparaissent réalisables avec la technologie actuelle. Évidemment, on ne peut prétendre que ces différentes suggestions soient une solution finale aux problèmes auxquels ils s'adressent. On peut toutefois espérer qu'ils représentent un pas de plus dans la bonne direction.

Avant de pouvoir réaliser un ordinateur quantique utile basé sur les concepts présentés ici, beaucoup de travail expérimental et théorique reste à faire. En particulier, il semble nécessaire de regarder en plus grand détail le problème de la fuite hors des états logiques de la CBJJ lors de l'interaction qubit-qubit. Des solutions algorithmiques à ce genre de problème ont déjà été étudiées mais il reste à les appliquer à la situation qui nous intéresse ici. De plus, un des concepts récents le plus important pour le domaine est probablement l'utilisation d'un point d'opération où le qubit n'est pas sensible aux fluctuations de ces paramètres de contrôle. Ce type de point particulier a été utilisé avec succès dans l'expérience du qubit phase-charge [6] et il est aussi présent dans le détecteur discuté au chapitre 5. Dans la recherche de nouveaux types de qubits, de détecteurs et même de méthodes d'interaction qubit-qubit, il semble primordial d'avoir ce concept à l'esprit.

Finalement, au cours des trois dernières années, le domaine est passé de presque purement théorique à un domaine mené par l'expérience. Ce changement de cap montre une confiance dans les chances de succès de cette technologie. Il apparaît toutefois difficile de prévoir quand sera réalisé un ordinateur quantique pouvant supplanter les ordinateurs classiques dans l'exécution de calculs utiles, si un tel ordinateur est jamais réalisé. Malgré ces incertitudes, le chemin vers la réalisation d'un ordinateur quantique est parsemé de physique passionnante et son exploration vaut les efforts investis. 


\section{Annexe A}

\section{Phase géométrique sur qubit de charge}

Dans cette annexe, on commence par reproduire les résultats de Falci et al. concernant la phase géométrique adiabatique sur les qubits de charge [83]. Il s'agit d'un exemple intéressant de phase géométrique. Ensuite, la procédure pour réaliser la phase géométrique non-adiabatique à l'aide de ce même type de qubit est présentée.

\section{A.1 Phase géométrique adiabatique}

Afin d'appliquer la phase de Berry au calcul quantique, il est nécessaire d'avoir un contrôle sur les trois champs effectifs de l'Hamiltonien à un qubit. Pour arriver à un tel contrôle, Falci et al. ont étudié le qubit de charge asymétrique. Ici, l'asymétrie se trouve entre les jonctions Josephson formant la boucle du qubit. Tel que décrit au chapitre 3 , l'Hamiltonien de ce système est

$$
H=4 E_{C}\left(n-n_{g}\right)^{2}-E_{J 1} \cos \varphi_{1}-E_{J 2} \cos \varphi_{2},
$$

où $n$ est le nombre de paires de Cooper sur l'île de la boîte, $n_{g}$ la charge de grille adimensionnelle, $E_{C}$ l'énergie de charge et $E_{J i}$ l'énergie Josephson de la jonction $i$ ayant une différence de phase $\varphi_{i}$.

Avec $\phi=\varphi_{1}-\varphi_{2}$ et pour une boucle de faible inductance, on peut réecrire l'Hamiltonien du qubit asymétrique sous la forme

$$
H=4 E_{C}\left(n-n_{g}\right)^{2}-E_{J}\left(\Phi_{x}\right) \cos (\phi-\alpha)
$$




\section{A.1. PHASE GÉOMÉTRIQUE ADIABATIQUE}

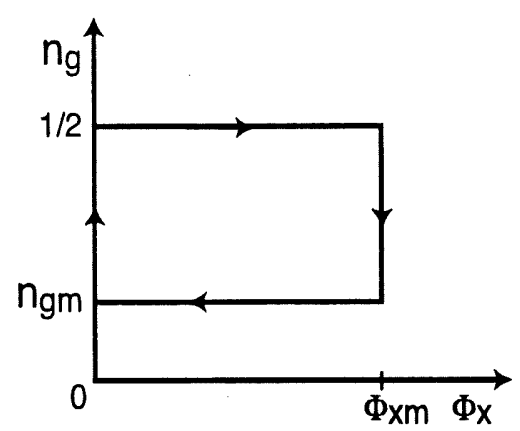

Frg. A.1: Circuit dans l'espace des paramètres $\left\{\Phi_{x}, n_{g}\right\}$.

où

$$
E_{J}\left(\Phi_{x}\right)=\sqrt{\left(E_{J 1}-E_{J 2}\right)^{2}+4 E_{J 1} E_{J 2} \cos ^{2}\left(\pi \frac{\Phi_{x}}{\Phi_{0}}\right)}
$$

et

$$
\tan \alpha=\frac{E_{J 1}-E_{J 2}}{E_{J 1}+E_{J 2}} \tan \left(\pi \frac{\Phi_{x}}{\Phi_{0}}\right) .
$$

Avec le remplacement usuel vers les matrice de Pauli, on peut réécrire ce résultat sous la forme

$$
H=\frac{1}{2} \boldsymbol{B} \cdot \boldsymbol{\sigma}
$$

avec comme champ effectif $B=\left\{E_{J} \cos \alpha,-E_{J} \sin \alpha, 4 E_{C}\left(1-2 n_{g}\right)\right\}$. Sans l'asymétrie, $\alpha=0$ et $B_{y}$ est fixé à zéro.

Considérons maintenant l'évolution du système sous la variation adiabatique des paramètres de l'Hamiltonien sur le parcours fermé $C$ de la figure A.1. Tel que décrit au chapitre 2, suite à ce parcours les états propres de l'Hamiltonien auront une phase dynamique et une phase géométrique. Cette dernière est proportionelle à l'angle solide soutenu par $\tilde{C}$ au point de dégénérescence $B=0$. Ici, $\tilde{C}$ est la projection de $C$ sur la sphère. L'angle solide est donné par

$$
\Omega(\tilde{C})=\iint_{\tilde{C}} d \varphi d \theta \sin \theta
$$

où $\theta$ et $\varphi$ sont

$$
\cos \theta=\frac{B_{z}}{B}, \quad \tan \varphi=\frac{B_{y}}{B_{x}}
$$




\section{A.1. PHASE GÉOMÉTRIQUE ADIABATIQUE}

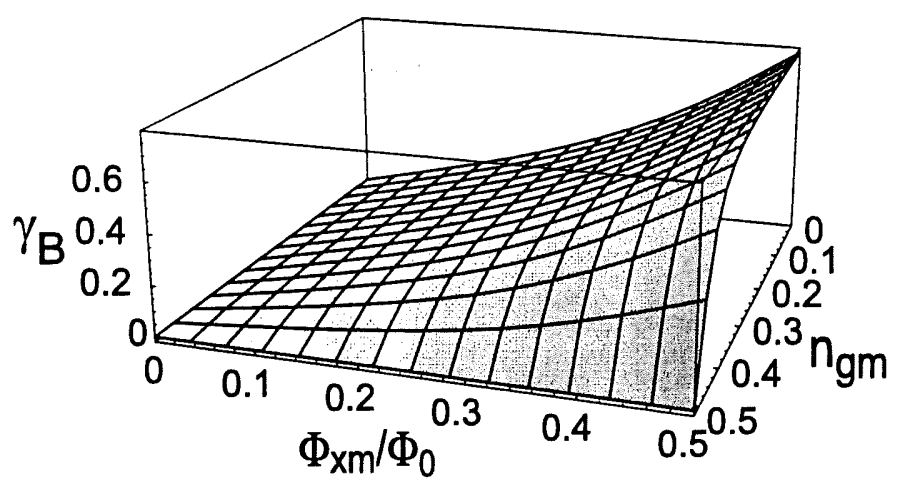

FIG. A.2: Phase de Berry en fonction $n_{g m}$ et $\Phi_{x m}$. Les valeurs pour les différents paramètres sont les mêmes qu'à la référence [83], soit $E_{J 1}=E_{J 2} / 4$ et $E_{C}=5\left(E_{J 1}+E_{J 2}\right)$.

et $B=\sqrt{B_{x}^{2}+B_{y}^{2}+B_{z}^{2}}$.

La relation (A.6) s'intègre plus facilement dans l'espace $\left\{n_{x}, \Phi\right\}$. On a alors

$$
\Omega(\tilde{C})=\int_{n_{g m}}^{1 / 2} \int_{0}^{\Phi_{x m}} d n_{g} d \Phi_{x} J\left(n_{g}, \Phi_{x}\right) \sqrt{1-\left(\frac{B_{z}\left(n_{g}\right)}{B\left(n_{g}, \Phi_{x}\right)}\right)^{2}},
$$

où $J\left(n_{g}, \Phi_{x}\right)$ est le Jacobien de la transformation. Après intégration, on obtient

$$
\Omega\left(n_{g m}, \Phi_{x m}\right)=\frac{2 E_{C}\left(1-2 n_{g m}\right)}{4\left(E_{J 1} E_{J 2}\right)^{(1 / 2)}} \lambda \mu \Pi\left(\pi \frac{\Phi_{x m}}{\Phi_{0}}, 1-\mu^{2}, \lambda\right)
$$

avec

$$
\begin{aligned}
\mu & \equiv \frac{E_{J 1}-E_{J 2}}{E_{J 1}+E_{J 2}} \\
\lambda^{2} & \equiv \frac{4\left(E_{J 1} E_{J 2}\right)^{(1 / 2)}}{E_{C}^{2}\left(1-2 n_{g m}\right)^{2}+\left(E_{J 1}+E_{J 2}\right)^{2}},
\end{aligned}
$$

et où $\Pi(x, n, k)$ est l'intégrale elliptique du troisième type. La figure A.2 présente ce résultat en fonction de $n_{g m}$ et $\Phi_{x m}$.

Puisque l'on s'intéresse à un pseudo-spin $1 / 2$, la phase de Berry pour les états propres est $\gamma_{B}= \pm \frac{1}{2} \Omega$. En débutant l'évolution avec une superposition des états propres, on obtient une phase géométrique relative $2 \gamma_{B}$ à la fin de l'évolution [83]. Afin d'avoir une phase purement géométrique, il est nécessaire d'annuler la contribution dynamique par écho de spin. 


\section{A.2 Phase géométrique non-adiabatique}

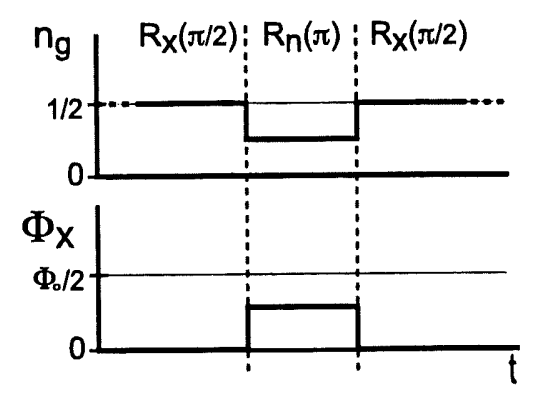

FIG. A.3: Séquence de flux externe $\Phi_{x}$ et de charge de grille $n_{g}$ réalisant l'opération $R_{z}^{A A}(\theta)$ sur un qubit de charge. L'amplitude relative du flux et du voltage de grille pour la porte $R_{n}(\pi)$ détermine $\theta$, voir figure A.4.

La phase géométrique ne nécessite que deux champs de contrôle. Elle peut donc être réalisée, par exemple, à l'aide d'un qubit de charge symétrique. Dans ce système, on réalise l'opération $R_{z}^{A A}(\theta)$ de la section $\S 2.1 .3$ à l'aide de la séquence de voltage de grille et du flux extérieur présentée à la figure A.3. Les figures A.4 a) et A.4 b) présentent respectivement l'angle $\theta$ et l'amplitude du champ effectif $B_{n}$ générant $R_{n}(\pi)$ en fonction du voltage de grille et du flux extérieur sur le qubit de charge. En raison de la dépendance de $B_{n}$ sur ces paramètres de contrôle, le temps $t_{n}=\pi / B_{n}$ nécessaire pour réaliser la porte $R_{n}(\pi)$ dépend de la phase géométrique $\theta$ voulue, figure A.4 c). 

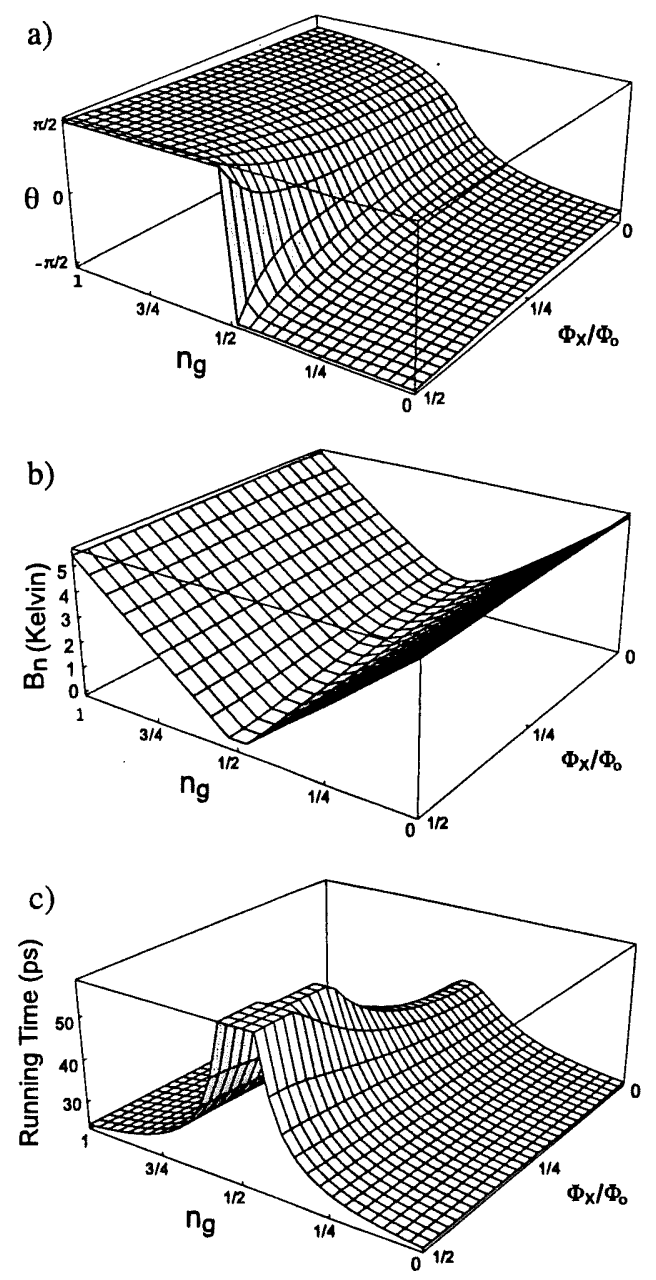

Fig. A.4: a) Valeurs possibles de la phase géométrique $\theta=$ $\arctan \left[2 E_{C}\left(2 n_{g}-1\right) / E_{J} \cos \left(\pi \Phi_{x} / \Phi_{0}\right)\right]$ pour le qubit de charge symétrique en fonction des paramètres extérieurs générant la porte $R_{n}(\pi)$. Les énergies du qubit sont choisies ici comme à la référence [144] $: E_{J}=0.6 \mathrm{~K}$ et $E_{C}=1.35 \mathrm{~K}$. La phase relative $2 \theta$ peut être choisie dans la gamme complète de valeurs $[0,2 \pi]$ par un choix approprié des paramètres de contrôle. b) Grandeur du champ effectif $B_{n}$ en fonction des paramètres extérieurs. c) Temps nécessaire à la réalisation de $R_{z}^{A A}(\theta)$ (en picosecondes) en fonction des paramètres générant $R_{n}(\pi)$. On suppose ici que les portes $R_{x}(\pi / 2)$ dans (2.15) sont réalisées le plus rapidement possible en fonction des énergies données plus haut. En raison des limitations des sources de voltage et de courant (i.e. flux), ce temps d'opération peut être plus long [144]. Le temps de montée des impulsions de voltage/flux n'a pas été pris en considération ici. 


\section{Annexe B}

\section{Distance pour opérations dynamiques}

\section{bruyantes}

Dans cette annexe, on présente les principales étapes de calcul nécessaires afin d'obtenir la distance $D(\mathrm{U}, \mathrm{V})$ pour les portes dynamiques avec bruit $\mathrm{V}=\tilde{\mathrm{R}}_{\mathbf{z}}(\theta)$ par rapport aux portes dynamiques idéales $U=\mathrm{R}_{\mathbf{z}}(\theta)=e^{-i B_{z} \sigma_{z} t / 2}$. Tel qu'exprimé par la relation (2.23), le bruit est modélisé en ajoutant simplement une composante aléatoire $\delta B_{i}(t) \sigma_{i}$ à l'Hamiltonien sans fluctuations $B_{z} \sigma_{z} / 2$. Ensuite, utilisant la propriété de composition de l'opérateur d'évolution (2.24), on écrit pour les opérations avec bruit

$$
\mathrm{V}=\prod_{n=1}^{N} e^{-i\left(B_{z}+\delta B_{z}^{n}\right) \frac{\Delta t}{2} \sigma_{z}-i \delta B_{x}^{n} \frac{\Delta t}{2} \sigma_{x}}
$$

où $\Delta t=t / N$ est défini comme étant le temps de corrélation du bruit. Au premier ordre en $\Delta t$ et $\delta B$, on peut réécrire cette expression de la façon suivante :

$$
\mathrm{V} \approx \prod_{n=1}^{N} e^{-i\left(B_{z}+\delta B_{z}^{n}\right) \frac{\Delta t}{2} \sigma_{z}} e^{-i \delta B_{x}^{n} \frac{\Delta t}{2} \sigma_{x}}
$$

Ensuite, en développant les exponentielles au premier ordre en $\Delta t$ et $\delta B$ on obtient

$$
\mathrm{V} \approx e^{-i B_{z} \frac{t}{2} \sigma_{z}}\left(\mathbb{I}-i \frac{\Delta t}{2} \sigma_{z} \sum_{n=1}^{N} \delta B_{z}^{n}-i \frac{\Delta t}{2} \sigma_{x} \sum_{n=1}^{N} \delta B_{x}^{n}\right)
$$

où nous avons encore utilisé le fait que les commutateurs impliqués s'annulent au premier ordre en temps $\Delta t$ et en bruit $\delta B$. 
À l'aide de cette approximation pour $\mathrm{V}$, il est facile de calculer la distance

$$
\begin{aligned}
D(\mathrm{U}, \mathrm{V}) & \approx \operatorname{Tr} \sqrt{\left[\left(\frac{\Delta t}{2} \sum_{n=1}^{N} \delta B_{z}^{n}\right)^{2}+\left(\frac{\Delta t}{2} \sum_{n=1}^{N} \delta B_{x}^{n}\right)^{2}\right] \mathbb{I}} \\
& =\Delta t \sqrt{X_{z}^{2}+X_{x}^{2}}
\end{aligned}
$$

avec $X_{i} \equiv \sum_{n=1}^{N} \delta B_{i}^{n}$.

À l'aide du théorème de la limite centrale, on prend maintenant la moyenne de ce résultat sur $M$ réalisations du bruit. Puisque les $\delta B_{i}$ sont des variables indépendantes tirées d'une distribution de probabilité uniforme dans l'intervalle $\pm \delta B_{\max }$, on a $\left\langle X_{i}\right\rangle=$ 0 . Pour l'écart type de ces variables, on obtient

$$
\begin{aligned}
\left\langle\Delta X_{i}^{2}\right\rangle & =N \Delta t^{2}\left\langle\delta B^{2}\right\rangle \\
& =N \Delta t^{2} \int_{-\delta B_{\max }}^{\delta B_{\max }} \frac{d(\delta B)}{2 \delta B_{\max }} \delta B^{2} \\
& =\Delta t^{2} \frac{N \delta B_{\max }^{2}}{3} \\
& \equiv \sigma .
\end{aligned}
$$

La moyenne de la distance est alors simplement

$$
\begin{aligned}
\langle D(\mathrm{U}, \mathrm{V})\rangle & \approx \Delta t\left\langle\sqrt{X_{z}^{2}+X_{x}^{2}}\right\rangle \\
& =\frac{\Delta t}{2 \pi \sigma^{2}} \iint_{-\infty}^{\infty} d X_{x} d X_{z} \sqrt{X_{z}^{2}+X_{x}^{2}} e^{\frac{-x_{z}^{2}}{2 \sigma^{2}}} e^{\frac{-x_{x}^{2}}{2 \sigma^{2}}} \\
& =\frac{\Delta t}{2 \pi \sigma^{2}} \int_{0}^{2 \pi} d \theta \int_{0}^{\infty} d r r^{2} e^{\frac{-r^{2}}{2 \sigma^{2}}} \\
& =\sqrt{\frac{\pi}{2}} \Delta t \sqrt{\frac{N \delta B_{\max }^{2}}{3}}
\end{aligned}
$$

où le remplacement $X_{z}=r \cos \theta, X_{x}=r \sin \theta$ a été fait avant de réaliser l'intégrale Gaussienne. En utilisant $t=N \Delta t$ et $\theta=B_{z} t$ on obtient finalement l'expression $(2.27) \mathrm{b})$ pour la distance moyenne.

Obtenir l'expression équivalente (2.27) a) pour la phase AA est plus fastidieux mais se fait avec les mêmes étapes. Pour obtenir cette expression, on doit tenir compte du fait que les opérations composant $\mathrm{R}_{\mathrm{AA}}(\theta / 2)$ prennent un temps différent pour être 
réalisées. Afin de garder le temps de corrélation constant sous toute la séquence, on choisit donc un nombre d'intervalles différents pour la décomposition (2.24) de chacune des opérations. Il s'agit de la seule subtilité supplémentaire. 


\section{Annexe C}

\section{Encodage et suppression d'erreurs pour qubits de phase supraconducteurs}

Dans cette annexe, on reproduit un article réalisé en collaboration avec Daniel A. Lidar et Lian-Ao Wu de l'Université de Toronto. Dans ce travail, on utilise le concept d'universalité encodée afin de simplifier les designs de qubits de phase supraconducteurs. On s'intéresse plus particulièrement aux qubits basés sur les barrières de grain entre supraconducteurs de type $d[5,160]$. On réussit ainsi à se débarrasser d'une opération difficile à réaliser en pratique dans ces systèmes : la rotation $R_{z}(\phi)$.

Deux versions du code sont proposées. Une première requérant $2 N$ qubits physiques pour encoder $N$ qubits logiques et une seconde version ne requérant que $N+1$ qubits physiques. Cette dernière approche est basée sur l'utilisation d'un 'bus qubit' fixé dans un état logique. Malgré le choix d'un nom similaire, ce bus n'est pas utilisé de la même façon que dans les architectures basées sur les pièges linéaires à ions.

La fuite hors du sous-espace du code est étudiée en utilisant, en autres, les techniques de recouplage ('recoupling'). De plus, la technique bang-bang de réduction d'erreurs, déjà étudiée à la référence [5], est explorée en plus de détails.

Mes contributions principales dans ce travail ont été de suggérer le code ne requérant que $N+1$ qubits physiques et de faire le lien entre les codes et les architectures de qubits supraconducteurs. 
Pour l'article complèt voir la copie papier à la Bibliothèque du Frère-Théode Section Monographie QA 76.889 B528 2002

Quantum Information Processing, Vol. 1, No. 3, June 2002 ((C) 2002)

\title{
Quantum Codes for Simplifying Design and Suppressing Decoherence in Superconducting Phase-Qubits
}

\author{
Daniel A. Lidar, ${ }^{1,3}$ Lian-Ao Wu, ${ }^{1}$ and Alexandre Blais ${ }^{2}$
}

Received April 4, 2002: accepted June 16, 2002

We introduce simple qubit-encodings and logic gates which eliminate the need for certain difficult single-qubit operations in superconducting phase-qubits, while preserving universality. The simplest encoding uses two physical qubits per logical qubit. Two architectures for its implementation are proposed: one employing $N$ physical qubits out of which $N / 2$ are ancillas fixed in the |1) state, the other employing $N / 2+1$ physical qubits, one of which is a bus qubit connected to all others. Details of a minimal set of universal encoded logic operations are given, together with recoupling schemes, that require nanosecond pulses. A generalization to codes with higher ratio of number of logical qubits per physical qubits is presented. Compatible decoherence and noise suppression strategies are also discussed.

KEY WORDS: quantum computation; superconducting phase-qubits; superconducting junction devices; quantum codes; dynamical decoupling; decoherence.

PACS: 03.67.Lx; 85.25.Hv; 03.67.-a; 89.70. +c. 


\section{Annexe D}

\section{Qubits à terminaux multiples}

On reproduit ici un article concernant un nouveau type de qubit supraconducteur. Ce travail a été réalisé en collaboration avec les chercheurs de D-Wave Systems inc., compagnie basée à Vancouver, et A.N. Omelyanchouk de l'Institue B.I. Verkin en Ukraine.

Mes contributions sont principalement au niveau des opérations logiques et du calcul des temps de décohérence due aux fluctuations des paramètres de contrôle. L'idée originale pour ce design de qubit a été proposée par M.H.S. Amin.

Une version plus complète de cet article est en préparation et sera, éventuellement. soumise pour publication. Ce manuscrit comprend, en autre, une discussion de l'utilisation des DFS avec ce type de qubit et une façon originale de manipuler le temps de décohérence du système selon que l'on cherche à initialiser un qubit (et donc avoir un cours temps de cohérence) ou à le manipuler de façon cohérente. Cette idée est basée sur les travaux expérimentaux de M. Watanabe et al. [228]. Toutefois, des idées similaires mais en relation avec les qubits de charge ont depuis été publiées par d'autres auteurs [229]. 
Pour l'article complet voir la copie papier à la Bibliothèque du Frère-Théode Section Monographie QA 76.889 B528 2002

$\mathrm{N} \cdot \mathrm{H}$

\section{Multi-terminal superconducting phase qubit}

M.H.S. Amin ${ }^{\mathrm{a}, *}$, A.N. Omelyanchouk ${ }^{\mathrm{b}}$, A. Blais ${ }^{\mathrm{c}}$, Alec Maassen van den Brink ${ }^{\mathrm{a}}$, G. Rose ${ }^{\mathrm{a}}$, T. Duty ${ }^{\mathrm{a}}$, A.M. Zagoskin ${ }^{\mathrm{a}, \mathrm{d}}$

${ }^{a} D$-Wave Systems Inc., 320-1985 West Broadway, Vancouver, BC, Canada V6J 4 Y3

b B.I.Verkin Institute for Low Temperature Physics and Engineering, Ukrainian National Academy of Sciences, Lenin Ave. 47, Kharkov 310164, Ukraine

c Centre de Recherche sur les Propriétés Électroniques de Matériaux Avancés and Département de Physique, Université de Sherbrooke, Sherbrooke, Québec, Canada JIK 2RI

d Department of Physics and Astronomy. The University of British Columbia, 6224 Agricultural Road, Vancouver, BC, Canada V6T IZ1 


\section{Annexe E}

\section{Taux de déphasage et de relaxation}

On obtient dans cette annexe les taux de déphasage et de relaxation au premier ordre en théorie de perturbation. Pour le taux de relaxation, ce calcul est présenté de façon très pédagogique à la référence [134]. On emprunte ici la notation et l'approche de cet article afin d'obtenir le taux de déphasage.

Comme à la section $\S 3.3$, on se place dans la base des états propres de l'hamiltonien à un qubit. Par définition, dans cette base ce dernier s'exprime comme

$$
H_{0}=-\frac{\hbar \omega_{0}}{2} \sigma_{z}
$$

où $\hbar \omega_{0}$ est la séparation en énergie entre les états propres du qubit et les $\sigma_{i}$ sont ici les matrices de Pauli dans la base des états propres $\{|0\rangle,|1\rangle\}$ de $H_{0}{ }^{1}$. On suppose le qubit couplé à des sources de bruit $f_{i}(t)$ dans les directions $x$ et $z$. La perturbation prend donc la forme :

$$
V=\eta_{x} f_{x}(t) \sigma_{x}+\eta_{z} f_{z}(t) \sigma_{z}
$$

où les $\eta_{i}$ sont des constantes de couplage.

Afin de déterminer la relaxation, on s'intéresse au taux de transition entre les états propres de $H_{0}$. On s'intéresse toutefois ici au déphasage et on cherchera plutôt à obtenir le taux de transition entre les superpositions $| \pm\rangle=(|0\rangle \pm|1\rangle) / \sqrt{2}$. Une telle transition correspond à un renversement complet de la phase. Si le système est préparé

\footnotetext{
${ }^{1}$ On note ici les états propres $\{|0\rangle,|1\rangle\}$. Il est important de garder à l'esprit que ces états propres seront généralement des superpositions des états logiques.
} 
initialement dans l'état $|\psi(0)\rangle=|-\rangle$, celui-ci sera, au premier ordre de perturbation, dans l'état

$$
|\hat{\psi}(t)\rangle=|\psi(0)\rangle-\frac{i}{\hbar} \int_{0}^{t} d \tau \hat{V}(\tau)|\psi(0)\rangle
$$

au temps $t$. On travaille ici en représentation d'interaction :

$$
\hat{V}(\tau)=e^{i H_{0} \tau / \hbar} V e^{-i H_{0} \tau / \hbar}
$$

De (E.2) et (E.3), on obtient pour l'amplitude de transition $\alpha_{\mp}$ entre les états $|-\rangle$ et $|+\rangle$

$$
\begin{aligned}
\alpha_{\mp} & =-i \frac{\eta_{x}}{\hbar} \int_{0}^{t} d \tau\left\langle+\left|\hat{\sigma}_{x}(\tau)\right|-\right\rangle f_{x}(\tau)-i \frac{\eta_{z}}{\hbar} \int_{0}^{t} d \tau\left\langle+\left|\hat{\sigma}_{z}(\tau)\right|-\right\rangle f_{z}(\tau)+O\left(\eta_{x, z}^{2}\right) \\
& =\frac{\eta_{x}}{\hbar} \int_{0}^{t} d \tau \sin \left(\omega_{0} \tau\right) f_{x}(\tau)-i \frac{\eta_{z}}{\hbar} \int_{0}^{t} d \tau f_{z}(\tau)+O\left(\eta_{x, z}^{2}\right),
\end{aligned}
$$

où on a utilisé le fait que $e^{i H_{0} t / \hbar} \sigma_{x}=\sigma_{x} e^{-i H_{0} t / \hbar}$ afin de simplifier le premier membre de droite et $\left[H_{0}, \sigma_{z}\right]=0$ pour le second. De ce résultat, on obtient pour la probabilité de transition

$$
\begin{aligned}
p_{\mp}(t) \equiv\left|\alpha_{\mp}\right|^{2}= & \frac{\eta_{x}^{2}}{\hbar^{2}} \int_{0}^{t} \int_{0}^{t} d \tau_{1} d \tau_{2} \sin \left(\omega_{0} \tau_{1}\right) \sin \left(\omega_{0} \tau_{2}\right) f_{x}\left(\tau_{1}\right) f_{x}\left(\tau_{2}\right) \\
& +\frac{\eta_{z}^{2}}{\hbar^{2}} \int_{0}^{t} \int_{0}^{t} d \tau_{1} d \tau_{2} f_{z}\left(\tau_{1}\right) f_{z}\left(\tau_{2}\right)+O\left(\eta_{x, z}^{3}\right) .
\end{aligned}
$$

De la même façon qu'à l'annexe $\mathrm{B}$, on s'intéresse à la moyenne sur différentes réalisations du bruit :

$$
\begin{aligned}
\bar{p}_{\mp}(t)= & \frac{\eta_{x}^{2}}{\hbar^{2}} \int_{0}^{t} \int_{0}^{t} d \tau_{1} d \tau_{2} \sin \left(\omega_{0} \tau_{1}\right) \sin \left(\omega_{0} \tau_{2}\right)\left\langle f_{x}\left(\tau_{1}\right) f_{x}\left(\tau_{2}\right)\right\rangle \\
& +\frac{\eta_{z}^{2}}{\hbar^{2}} \int_{0}^{t} \int_{0}^{t} d \tau_{1} d \tau_{2}\left\langle f_{x}\left(\tau_{1}\right) f_{x}\left(\tau_{2}\right)\right\rangle+O\left(\eta_{x, z}^{3}\right) .
\end{aligned}
$$

Suivant toujours la référence [134], on procède maintenant au changement de variables suivant : $\tau=\tau_{1}-\tau_{2}$ et $T=\left(\tau_{1}+\tau_{2}\right) / 2$. La probabilité de transition s'exprime alors comme

$$
\begin{aligned}
\bar{p}_{\mp}(t)= & -\frac{1}{2} \frac{\eta_{x}^{2}}{\hbar^{2}} \int_{0}^{t} d T \int_{-B(T)}^{B(T)} d \tau\left\{\cos \left(2 \omega_{0} T\right)-\cos \left(\omega_{0} \tau\right)\right\}\left\langle f_{x}(T+\tau / 2) f_{x}(T-\tau / 2)\right\rangle \\
& +\frac{\eta_{z}^{2}}{\hbar^{2}} \int_{0}^{t} d T \int_{-B(T)}^{B(T)} d \tau\left\langle f_{x}(T+\tau / 2) f_{x}(T-\tau / 2)\right\rangle+O\left(\eta_{x, z}^{3}\right),
\end{aligned}
$$


où

$$
\begin{aligned}
& B(T)=T \quad \text { si } \quad T<t / 2 \\
& =t-T \quad \text { si } \quad T>t / 2 \text {. }
\end{aligned}
$$

On suppose maintenant que les fonctions de corrélation du bruit sont invariantes sous translation du temps. On suppose aussi qu'elles ont un petit temps d'autocorrélation $\tau_{f}$. Si on s'intéresse aux temps beaucoup plus grands que $\tau_{f}$, on peut alors faire le remplacement $B(T) \rightarrow \infty$ dans les intégrales sur $\tau$. On obtient alors

$$
\begin{aligned}
\bar{p}_{\mp}(t)= & \frac{1}{2} \frac{\eta_{x}^{2}}{\hbar^{2}} \int_{0}^{t} d T \int_{-\infty}^{\infty} d \tau \cos \left(\omega_{0} \tau\right)\left\langle f_{x}(\tau) f_{x}(0)\right\rangle \\
& +\frac{\eta_{z}^{2}}{\hbar^{2}} \int_{0}^{t} d T \int_{-\infty}^{\infty} d \tau\left\langle f_{x}(\tau) f_{x}(0)\right\rangle+O\left(\eta_{x, z}^{3}\right)
\end{aligned}
$$

On a omit ici le terme oscillant en $\cos \left(2 \omega_{0} T\right)$. La fonction de corrélation $\left\langle f_{x}(\tau) f_{x}(0)\right\rangle$ ne dépend pas de $T$ de sorte que celui-ci s'annule en moyenne puisque l'on s'intéresse à des temps plus grand que l'inverse de la fréquence naturelle $1 / \omega_{0}$ du système. En utilisant la définition du spectre de fluctuation de $f_{i}(t)$

$$
S_{f_{i}}(\omega)=\int_{-\infty}^{\infty} d \tau e^{+i \omega \tau}\left\langle f_{i}(\tau) f_{i}(0)\right\rangle
$$

on peut finalement réécrire la probabilité de transition sous la forme

$$
\bar{p}_{\mp}(t)=t \frac{1}{4} \frac{\eta_{x}^{2}}{\hbar^{2}}\left(S_{f_{x}}\left(\omega_{0}\right)+S_{f_{x}}\left(-\omega_{0}\right)\right)+t \frac{\eta_{z}^{2}}{\hbar^{2}} S_{f_{z}}(\omega \rightarrow 0)+O\left(\eta_{x, z}^{3}\right) .
$$

En dérivant $\bar{p}_{\mp}(t)$ par rapport au temps, on obtient le taux de transition de $|-\rangle$ vers $|+\rangle$ :

$$
\Gamma_{\mp}=\frac{1}{4} \frac{\eta_{x}^{2}}{\hbar^{2}}\left(S_{f_{x}}\left(\omega_{0}\right)+S_{f_{x}}\left(-\omega_{0}\right)\right)+\frac{\eta_{z}^{2}}{\hbar^{2}} S_{f_{z}}(\omega \rightarrow 0) .
$$

Afin d'obtenir le temps de déphasage, on doit aussi tenir compte de la transition $|+\rangle \rightarrow|-\rangle$. En répétant les mêmes étapes on vérifie que le résultat est identique à (E.13). Le taux de déphasage est par conséquent :

$$
\begin{aligned}
\Gamma_{\phi} & =\Gamma_{\mp}+\Gamma_{ \pm} \\
& =\frac{1}{2} \frac{\eta_{x}^{2}}{\hbar^{2}}\left(S_{f_{x}}\left(\omega_{0}\right)+S_{f_{x}}\left(-\omega_{0}\right)\right)+\frac{\eta_{z}^{2}}{\hbar^{2}}\left(S_{f_{z}}(\omega \rightarrow 0)+S_{f_{z}}(\omega \rightarrow 0)\right) \\
& =\frac{1}{2} \frac{\eta_{x}^{2}}{\hbar^{2}} S_{f_{x}}^{s y m}\left(\omega_{0}\right)+\frac{\eta_{z}^{2}}{\hbar^{2}} S_{f_{z}}^{s y m}(\omega \rightarrow 0),
\end{aligned}
$$


où $S_{f_{i}}^{\text {sym }}(\omega) \equiv\left(S_{f_{i}}(\omega)+S_{f_{i}}(-\omega)\right)$ est le spectre symétrique de fluctuation. Cette distinction est importante puisque, malgré qu'on l'ait traitée jusqu'à présent comme une variable classique, la quantité $f$ est un opérateur agissant dans l'espace d'Hilbert de l'environnement. De ce fait, en général $[f(t), f(0)] \neq 0$ et donc $S_{f_{i}}(\omega) \neq S_{f_{i}}(-\omega)$. La référence [134] contient une discussion particulièrement intéressante de cette question. Notons que dans les chapitres de la thèse, on écrira simplement $S_{f_{i}}(\omega)$ pour $S_{f_{i}}^{s y m}(\omega)$.

Tel que mentionné en début de section, afin d'obtenir le taux de relaxation $\Gamma_{r}$, il suffit de répéter les mêmes étapes mais en s'intéressant plutôt aux transitions $|0\rangle \rightarrow|1\rangle$ et $|1\rangle \rightarrow|0\rangle$. Ceci est fait en détail à la référence [134] et le calcul ne sera pas répété ici. Mentionnons toutefois que dans ce cas, puisque $\left\langle 0\left|\hat{\sigma}_{z}(t)\right| 1\right\rangle=\left\langle 1\left|\hat{\sigma}_{z}(t)\right| 0\right\rangle=0$, seul le bruit selon $x$ participe à la relaxation. De ce fait, le résultat est simplement :

$$
\begin{aligned}
\Gamma_{r} & =\Gamma_{0 \rightarrow 1}+\Gamma_{1 \rightarrow 0} \\
& =\frac{\eta_{x}^{2}}{\hbar^{2}} S_{f_{x}}^{s y m}\left(\omega_{0}\right) .
\end{aligned}
$$

En portant ce dernier résultat dans l'expression pour $\Gamma_{\phi}$, on peut finalement résumer les résultats importants de cette annexe :

$$
\begin{aligned}
& \Gamma_{r}=\frac{\eta_{x}^{2}}{\hbar^{2}} S_{f_{x}}^{s y m}\left(\omega_{0}\right) \\
& \Gamma_{\phi}=\frac{\Gamma_{r}}{2}+\frac{\eta_{z}^{2}}{\hbar^{2}} S_{f_{z}}^{s y m}(\omega \rightarrow 0) .
\end{aligned}
$$




\section{Bibliographie}

[1] M.A. Nielsen et I.L. Chuang. Quantum Computation and Quantum Information. Cambridge University Press, 2000.

[2] E. Knill, R. Laflamme, R. Martinez, et C.-H. Tseng. An algorithmic benchmark for quantum information processing. Nature, $404: 368,2000$.

[3] W.S. Warren. The usefulness of NMR quantum computing. Science, 277 :1688, 1997.

[4] A. Blais. Calcul quantique universel sur qubits supraconducteurs. Mémore de Maîtrise., 1999.

[5] A. Blais et A.M. Zagoskin. Operation of universal gates in a solid-state quantum computer based on clean Josephson junctions between d-wave superconductors. Phys. Rev. A, 61 :042308, 2000.

[6] D. Vion, A. Aassime, A. Cottet, P. Joyez, H. Pothier, C. Urbina, D. Esteve, et M.H. Devoret. Manipulating the quantum state of an electrical circuit. Science, 296 :886, 2002.

[7] J.M. Martinis, S. Nam, J. Aumentado, et C. Urbina. Rabi oscillations in a large Josephson junction qubit. Phys. Rev. Lett., 89 :117901, 2002.

[8] Y. Yu, S. Han, X. Chu, S.-I. Chu, et Z. Wang. Coherent temporal oscillations of macroscopic quantum states in a Josephson junction. Science, 296 :889, 2002.

[9] B. Schumacher. Quantum coding. Phys. Rev. A, 51 :2738, 1995.

[10] A.S. Holevo. Capacity of quantum communication channel. Problems of Inf. Transm., 5 :247, 1979.

[11] S. Popescu et D. Rohrlich. Thermodynamics and the measure of entanglement. Phys. Rev. A, 56 :R3319, 1997. 
[12] A. Peres. Separability criterion for density matrices. Phys. Rev. Lett., $77: 1413$, 1996.

[13] W.K. Wootters. Entanglement of formation and concurrence. Quant. Inf. and Comp., $1: 27,2001$.

[14] C. Bennett, D.P. DiVincenzo, J.A. Smolin, et W.K. Wootters. Mixed-state entanglement and quantum error correction. Phys. Rev. A, 54 :3824, 1996.

[15] A. Ekert et R. Jozsa. Quantum algorithms : entanlement enchanced information processing. Phil. Trans. Roy. Soc. Lond. A, 356 :1779, 1998.

[16] S.L. Braunstein, C.M. Caves, R. Jozsa, N. Linden, S. Popescu, et R. Schack. Separability of very noisy mixed states and implications for nmr quantum computing. Phys. Rev. Lett., 83 :1054, 1999.

[17] N. Linden et S. Popescu. Good dynamics versus bad kinematics : Is entanglement needed for quantum computation? Phys. Rev. Lett., 87 :047901, 2001.

[18] R. Fitzgerald. What really gives a quantum computer its power? Physics Today, page 20, January 2001.

[19] D. DiVincenzo. The physical implementation of quantum computation. Fortschritte der Physik, 48 :771, 2000.

[20] D.P. DiVincenzo. Dogma and heresy in quantum computing. In R.G. Clark, editor, Proceedings of the first international conference on experimental implementation of quantum computation, page 48. Rinton Press, 2001.

[21] W.H. Zurek. Decoherence and the transition from quantum to classical. Physics Today, page 36, Octobre 1991.

[22] R.P. Feynman. Feynman Lectures on Computation. Addison-Wesley, New York, 1996.

[23] A. Barenco, CH. Bennett, R. Cleve, D.P. DiVincenzo, N. Margolus, Shor P, T. Sleator, J.A. Smolin, et Harald Weinfurter. Elementary gates for quantum computation. Phys. Rev. A, 52 :3457, 1995.

[24] Michael J. Bremner, Christopher M. Dawson, Jennifer L. Dodd, Alexei Gilchrist, Aram W. Harrow, Duncan Mortimer, Michael A. Nielsen, et Tobias J. Osborne. 
A practical scheme for quantum computation with any two-qubit entangling gate. quant-ph/0207072, 2002.

[25] P. Oscar Boykin, Tal Mor, Matthew Pulver, Vwani Roychowdhury, et Farrokh Vatan. On universal and fault-tolerant quantum computing. In 40th Annual Symposium on Fondations of Computer Science, page 486, Los Alamitos, CA, 1999. IEEE Comput. Soc.

[26] A. Yu Kitaev. Quantum computations : algorithms and error correction. Russian Math. Surveys, 52 :1191, 1997.

[27] D. Gottesman. Theory of fault-tolerant quantum computation. Phys. Rev. A, $57: 127,1998$.

[28] R.R. Ernst, G. Bodenhausen, et A. Wokaun. Principles of Nuclear Magnetic Resonance in One and Two Dimensions. Clarendon Press, Oxford, 1987.

[29] P.W. Shor. Scheme for reducing decoherence in quantum computer memory. Phys. Rev. A, 52 :R2493, 1995.

[30] A.M. Steane. Error correcting codes in quantum theory. Phys. Rev. Lett., $77: 793,1996$.

[31] F.J. MacWilliams et N.J.A. Sloane. The theory of error-correcting codes. NorthHolland Publishing, 1978.

[32] R. Laflamme, C. Miquel, J.P. Paz, et W.H. Zurek. Perfect quantum error correcting code. Phys. Rev. Lett., 77 :198, 1996.

[33] E. Knill et R. Laflame. Theory of quantum error-correcting codes. Phys. Rev. $A, \mathbf{5 5}: 900,1997$.

[34] A.M. Steane. Simple quantum error-correctiong codes. Phys. Rev. A, 54 :4741, 1996.

[35] A. R. Calderbank, E. M Rains, P. W. Shor, et N. J. A. Sloane. Quantum error correction via codes over GF(4). IEEE Trans. on Inf. Theory, $44: 1369,1998$.

[36] P. Zanardi et M. Rasetti. Noiseless quantum codes. Phys. Rev. Lett., 79 :3306, 1997.

[37] D.A. Lidar, I.L. Chuang, et K.B. Whaley. Decoherence-free subspaces for quantum computation. Phys. Rev. Lett., 81 :2594, 1998. 
[38] J. Kempe, D. Bacon, D.A. Lidar, et K. B. Whaley. Theory of decoherence-free fault-tolerant universal quantum computation. Phys. Rev. A, 63 :042307, 2001.

[39] Daniel A. Lidar, Dave Bacon, Julia Kempe, et K. B. Whaley. Decoherencefree subspaces for multiple-qubit errors. I. characterization. Phys. Rev. A, $63: 022306,2001$.

[40] D.A. Lidar, D. Bacon, et K. B. Whaley. Concatenating decoherence-free subspaces with quantum error correcting codes. Phys. Rev. Lett., 82 :4556, 1999.

[41] P.G. Kwiat, A.J. Berglund, J.B. Altepeter, et A.G. White. Experimental verification of decoherence-free subspaces. Science, $290: 498,2000$.

[42] D. Kielpinski, V. Meyer, M.A. Rowe, C.A. Sackett, W.M. Itano, C. Monroe, et D.J. Wineland. A decoherence-free quantum memory using trapped ions. Science, 291 :1013, 2001.

[43] Lorenza Viola, Evan M. Fortunato, Marco A. Pravia, Emanuel Knill, Raymond Laflamme, et David G. Cory. Experimental realization of noiseless subsystems for quantum information processing. Science, $293: 2059,2001$.

[44] V. Privmana, I. D. Vagnerb, et G. Kventsel. Quantum computation in quantumHall systems. Phys. Lett. A, 239 :141, 1998.

[45] P.M. Platzman et M. I. Dykman. Quantum computing with electrons floating on liquid helium. Science, 284 :1967, 1999.

[46] D. Loss et D. P. DiVincenzo. Quantum computation with quantum dots. Phys. Rev. A, $57: 120,1998$.

[47] B.E. Kane. A silicon-based nuclear spin quantum computer. Nature, 393 :133. 1998.

[48] L.-A. Wu et D. A. Lidar. Power of anisotropic exchange interactions : Universality and efficient codes for quantum computing. Phys. Rev. A, 65 :042318, 2002.

[49] D. P. DiVincenzo, D. Bacon, J. Kempe, G. Burkard, et K. B. Whaley. Universal quantum computation with the exchange interaction. Nature, $2000: 339,2000$.

[50] D. Bacon, J. Kempe, D.P. DiVincenzo, D.A. Lidar, et K.B. Whaley. Encoded universality in physical implementations of a quantum computer. In R. Clark, 
editor, Proceedings of the 1st International Conference on Experimental Emplementations of Quantum Computation, page 257, Princeton, NJ, 2001. Rinton.

[51] L.-A. Wu et D.A. Lidar. Qubits as parafermions. J. Math. Phys., 43 :4506, 2001.

[52] D.A. Lidar, L.-A. Wu, et A. Blais. Quantum codes for simplifying design and suppressing decoherence in superconducting phase-qubits. Quant. Inf. Processing, $1: 155,2002$.

[53] Edward Farhi, Jeffrey Goldstone, Sam Gutmann, et Michael Sipser. Limit on the speed of quantum computation in determining parity. Phys. Rev. Lett., $81: 5442,1998$.

[54] D. Simon. On the power of quantum computation. In Proceedings of the 35th annual symposium on the foundations of computer science, page 116, Los Alamitos, CA, 1994. IEEE Press.

[55] Lov K. Grover. Quantum mechanics helps in searching for a needle in a haystack. Phys. Rev. Lett., 79 :325, 1997.

[56] R. Feynman. Quantum-mechanical computers. Found. Phys., 16 :507, 1986.

[57] S. Lloyd. Universal quantum simulators. Science, 273 :1073, 1996.

[58] A.Yu. Kitaev. Quantum measurements and the abelian stabilizer problem. quant-ph/9511026, 1995.

[59] Richard Cleve, Artur Ekert, Chiara Macchiavello, et Michele Mosca. Quantum algorithms revisited. Proc. Roy. Soc. London A, 454 :339, 1998.

[60] D. Coppersmith. An approximate fourier transform useful in quantum factoring, IBM Research Report No. RC19642. Technical report, IBM, 1994.

[61] P.W. Shor. Polynomial-time algorithms for prime factorisation and discrete logarithms on a quantum computer. SIAM J. Comp., 26 :1484, 1997.

[62] D.S. Abrams and S. Lloyd. Quantum algorithm providing exponentional speed increase for finding eigenvalues and eigenvectors. Phys. Rev. Lett., 83 :5162, 1999.

[63] A. Blais. Matrix diagonalization on a quantum computer. Technical report, Université de Sherbrooke, November 1999. 
[64] B.C. Travaglione et G.J. Milburn. Generation of eigenstate using the phaseestimation algorithm. Phys. Rev. A, $63: 032301,2001$.

[65] H. De Raedt, A. Hams, K. Michielsen, S. Miyashita, et K. Saito. Quantum statistical mechanics on a quantum computer. Prog. Theor. Phys., 138 :489, 2000.

[66] J. Kim, J.-S. Lee, et S. Lee. Implementing unitary operators in quantum computation. Phys. Rev. A, $61: 032312,2000$.

[67] A. Blais. Quantum network optimization. Phys. Rev. A, 64 :022312, 2001.

[68] C. Moore et M. Nilsson. Parallel quantum computation and quantum codes. SIAM Journal on Computing, $31: 799,1998$.

[69] R. Cleve et J. Watrous. Fast parallel circuits for the quantum fourier transform. In Proceedings of the 41st Annual IEEE Symposium on Foundations of Computer Science (FOCS '00), page 526, 2000.

[70] A. Saito, K. Kioi, Y. Akagi, N. Hashizume, et K. Ohta. Actual computational time-cost of the quantum fourier transform in a quantum computer using nuclear spins. quant-ph/0001113, 2000.

[71] L. B. Ioffe, V. B. Geshkenbein, M. V. Feigel'man, A. L. Fauchère, et G. Blatter. Quiet SDS Josephson junctions for quantum computing. Nature, 398 :679, 1999.

[72] J.E. Mooij, T. P. Orlando, L. Levitov, Lin Tian, Caspar H. van der Wal, et Seth Lloyd. Josphson persistent-current qubit. Science, 285 :1036, 1999.

[73] D.G. Cory, A.F. Fahmy, et T.F. Havel. Ensemble quantum computing by NMR spectroscopy. Proc. Natl. Acad. Sci., 94 :1634, 1997.

[74] N.A. Gershenfeld et I.L. Chuang. Bulk spin-resonance quantum computation. Science, 275 :351, 1997.

[75] R.B. Griffiths et C. Niu. Semiclassical fourier transform for quantum computation. Phys. Rev. Lett., 275 :351, 1996.

[76] S.M. Sait et H. Youssef. VLSI Physical Design Automation. IEEE Press, New York, 1995. 
[77] E. Aarts et J. Korst. Simulated Annealing and Boltzmann Machines. Wiley, New York, 1989.

[78] A. Shnirman, G. Schön, et Z. Hermon. Quantum manipulation of small Josephson junctions. Phys. Rev. Lett., 79 :2371, 1997.

[79] L. Viola et S. Lloyd. Dynamical suppression of decoherence in two-state quantum system. Phys. Rev. A, $58: 2733,1998$.

[80] M.V. Berry. Quantal phase factors accompanying adiabatic changes. Proc. R. Soc. Lond., A392 :45, 1994.

[81] J. Jones, V. Vedral, A. Ekert, et G. Castagnoli. Geometric quantum computation using nuclear magnetic resonance. Nature, $403: 869,2000$.

[82] A. Ekert, M. Ericsson, P. Hayden, H. Inamori, J.A. Jones, D.K.L. Oi, et V. Vedral. Geometric quantum computation. Journal of Modern Optics, 47 :2501, 2000 .

[83] G. Falci, R. Fazio, G. Massimo Palma, J. Siewert, et V. Vedral. Detection of geometric phases in superconducting nanocircuits. Nature, $407: 355,2000$.

[84] S.-L. Zhu et Z.D. Wand. Geometric quantum computation using superconducting nanocircuits. Physica C, 364-365:213, 2001.

[85] F. Wilczek et A. Zee. Appearance of gauge structure in simple dynamical systems. Phys. Rev. Lett., 52 :2111, 1984.

[86] P. Zanardi et M. Rasetti. Holonomic quantum computation. Phys. Lett. A, $264: 94,1999$.

[87] L.-M. Duan, J.I. Cirac, et P. Zoller. Geometric manipulation of trapped ions for quantum computation. Science, 292 :1965, 2001.

[88] M.-S. Choi. Geometric quantum computation on solid-state qubits. quantph/0111019, 2001.

[89] L. Faoro, J. Siewert, et R. Fazio. Non-äbelian phases, pumping, and quantum computation with Josephson junctions. cond-mat/0202217, 2002.

[90] Y. Aharonov et J. Anandan. Phase change during cyclic quantum evolution. Phys. Rev. Lett., 58 :1593, 1987. 
[91] W. Xiang-Bin et M. Keiji. Non-adiabatically detecting the geometric phase of the macroscopic quantum state with symmetric squid. quant-ph/0104127, 2001.

[92] W. Xiang-Bin et M. Keiji. Nonadiabatic detection of the geometric phase of the macroscopic quantum state with a symmetric squid. Phys. Rev. B, $65: 172508$, 2002.

[93] S.L. Zhu et Z.D. Wang. Implementation of universal quantum gates based on nonadiabatic geometric phases. Phys. Rev. Lett., 89 :097902, 2002.

[94] Xin-Qi Li, Li-Xiang Cen, Guo-Xiang Huang, Lei Ma, et YiJing Yan. Non-adiabatic geometric quantum computation with trapped ions. quant$\mathrm{ph} / 0204028$.

[95] Jiangfeng Du, Mingjun Shi, Jihui Wu, Xianyi Zhou, et Rongdian Han. Implementations of nonadiabatic geometric quantum computation using NMR. quant-ph/0207022.

[96] S.-L. Zhu et Z. D. Wang. Geometric phase shift in quantum computation using superconducting nanocircuits : Nonadiabatic effects. Phys. Rev. A, 66 :042322, 2002.

[97] S.-L. Zhu et Z. D. Wang. Universal quantum gates based on a pair of orthogonal cyclic states in nmr systems. quant-ph/0210027.

[98] A. Blais. Non-adiabatic geometric phases for quantum computation. Novembre 2000.

[99] A. Blais et A.-M. S. Tremblay. Effect of noise on geometric logic gates for quantum computation. Phys. Rev. A, accepté pour publication; quant-ph/0105006.

[100] J.W. Zwanziger, M. Koening, et A. Pines. Berry's phase. Annu. Rev. Phys. Chem., 41 :601, 1990.

[101] J. Anandan. The geometric phase. Nature, $360: 307,1992$.

[102] A. Shapere et F. Wilczek. Geometric phases in physics. World Scinetific, Singapore, 1989.

[103] J. Anandan et L. Stodolsky. Some geometrical considerations of Berry's phase. Phys. Rev. D, 35 :2597, 1987. 
[104] D.J. Moore. The calculation of nonadiabatic berry phases. Phys. Rep., 210 :1, 1991.

[105] C. Bouchiat et G.W. Gibbons. Non-integrable quantum phase in the evolution of a spin-1 system : a physical consequence of the non-trivial topology of the quantum state-space. J. Phys., 49 :187, 1988.

[106] J. Anandan. Non-adiabatic non-abelian geometric phase. Phys. Lett. A, $133: 171,1988$.

[107] D. Suter, K.T. Mueller, et A. Pines. Study of the Aharonov-Anandan quantum phase by NMR interferometry. Phys. Rev. Lett., $60: 1218,1988$.

[108] Y. Makhlin, G. Schön, et A. Shnirman. Quantum-state engineering with Josephson-junction devices. Rev. Mod. Phys. Phys., 73 :357, 2001.

[109] M.A. Nielsen. A simple formula for the average gate fidelity of a quantum dynamical operation. quant-ph/0205035, 2002.

[110] A. Nazir, T.P. Spiller, et W.J. Munroe. Decoherence of geometric phase gates. Phys. Rev. A, 65 :042303, 2002.

[111] A.Yu Kitaev. Fault-tolerant quantum computation by anyons. quantph/9707021, 1997.

[112] L. B. Ioffe, M.V. Feigel'man, A. Ioselevich, D. Ivanov, M. Troyer, et G. Blatter. Topologically protected quantum bits from josephson junction arrays. Nature, $415: 503,2002$.

[113] L.B. Ioffe et M.V. Feigel'man. Possible realization of an ideal quantum computer in josephson junction array. cond-mat/0205186.

[114] P. Zanardi et S. Lloyd. Topological protection and noiseless subsystems. quantph/0208132, 2002.

[115] A.J. Leggett. Macroscopic quantum systems and the quantum theory of measurement. Supp. Prog. Theo. Phys., 69(80), 1980.

[116] T.P. Spiller. Superconducting circuits for quantum computing. Fortschr. Phys., $48: 1075,2000$. 
[117] M. Devoret. Quantum fluctuations in electrical circuits. In S. Reynaud, E. Giacobino, et J. Zinn-Justin, editors, Quantum fluctuations, page 351. Elsevier, 1997.

[118] M. Tinkham. Introduction to superconductivity. McGraw Hill, New York, 2 edition, 1996.

[119] P. Joyez. Le transistor à une paire de Cooper : un système quantique macroscopique. Thèse de Doctorat, Université Paris 6, 1995.

[120] H. Goldstein. Classical Mechanics. Addison-Wesley, 2 edition, 1980.

[121] T.P. Orlando, J.E. Mooij, L. Tian, C.H. van der Wal, L.S. Levitov, S. Lloyd, et J.J. Mazo. Superconducting persistent-current qubit. Phys. Rev. B, 60 :15398, 1999.

[122] K.A. Matveev, M. Gisselfält, L. I. Glazman, M. Jonson, et R. I. Shekhter. Parity-induced suppression of the Coulomb blockade of Josephson tunneling. Phys. Rev. Lett., 70 :2940, 1993.

[123] A.M. Zagoskin. Quantum theory of many-body systems. Spinger, New York, 1998.

[124] U. Weiss. Quantum Dissipative Systems. World Scientific, Singapore, 1999.

[125] Caspar H. van der Wal, F. K. Wilhelm, C. J. P. M. Harmans, et J. E. Mooi. Engineering decoherence in josephson persistent-current qubits : Measurement apparatus and other electromagnetic environments. cond-mat/0211664.

[126] A. Cottet, D. Vion, A. Assime, P. Joyez, D. Esteve, et M.H. Devoret. Implementation of a combined charge-phase quantum bit in a superconducting circuit. Physica C, 367 :197, 2002.

[127] R.P. Feynman et F.L. Vernon. The theory of a general quantum system interacting with a linear dissipative system. Ann. Phys., 24 :118, 1963.

[128] A.O. Caldeira et A.J. Leggett. Quantum tunneling in a dissipative system. Annals of Physics, 149 :374, 1983.

[129] A.J. Leggett. Quantum mechanics at the macroscopic level. In J. Souletie, J. Vannimenus, et R. Stora, editors, Chance and Matter, page 395, Amsterdam, 1987. North-Holland. 
[130] A.J. Leggett, S. Chakravarty, A.T. Dorsey, M.P.A. Fisher, A. Garg, et W. Zwerger. Dynamics of the dissipative two-state system. Rev. Mod. Phys., 59 :1, 1987.

[131] M. Grifoni, E. Paladino, et U. Weiss. Dissipation, decoherence and preparation effects in the spin-boson system. Eur. Phys. J. B, $10: 719,1999$.

[132] H. Schoeller et G. Schön. Mesoscopic quantun transport : resonant tunneling in the presence of strong Coulomb interaction. Phys. Rev. B, $50: 18436,1994$.

[133] A. Shnirman, Y. Makhlin, et G. Schön. Noise and decoherence in quantum two-level systems. cond-mat/0202518, 2002.

[134] R.J. Schoelkopf, A.A. Clerk, S.M. Girvin, K.W. Lehnert, et M.H. Devoret. Qubits as spectrometers of quantum noise. cond-mat/0210247, 2002.

[135] L. Tian, S. Lloyd, et T.P. Orlando. Decoherence and relaxation of a superconducting quantum bit during measurement. Phys. Rev. B, 65 :144516, 2002.

[136] T.P. Orlando, L. Tian, D.S. Crankshaw, S. Lloyd, C. H. van der Wal, J. E. Mooij, et F. Wilhelm. Engineering the quantum measurement process for the persistent current qubit. Physical C, 368 :294, 2002.

[137] D.P. DiVincenzo. Two-bit gates are universal for quantum computation. Phys. Rev. A, 51, 1995.

[138] J. Preskill. Reliable quantum computers. Proc. R. Soc. Lond. A, 454 :385. 1998.

[139] A.M. Steane. Efficient fault-tolerant quantum computing. Nature, 399 :124. 1999.

[140] L. Tian, L.S. Levitov, C.H. van der Wal, J.E. Mooij, T.P. Orlando, S. Lloyd. C.J. P.M. Harmans, et J.J. Mazo. Decoherence of the superconducting persistent current qubit. In I.Kulik et R.Elliatioglu, editors, Quantum Mesoscopic Phenomena and Mesoscopic Devices in Microelectronics, NATO-ASI Series E, page 429, Dordrecht, 2000. Kluwer Ac. Publ.

[141] A. Cottet, A. Steinbach, P. Joyez, D. Vion, H. Pothier, D. Esteve, et M. E. Huber. Superconducting electrometer for measuring the single Cooper pair box. In D.V. Averin, B. Ruggerio, et P. Silvestrini, editors, Proceedings of the International Workshop on Macroscopic Quantum Coherence and Quantum Computing, New York, 2001. Kluwer Academic/Plenum. 
[142] N. Prokof'ev et P. Stamp. Spin bath-mediated decoherence in superconductors. cond-mat/0001080.

[143] G. Rose et A.Yu. Smirnov. Effects of nuclear spins on the coherent evolution of a phase qubit. J. Phys. : Conds. Matter, 13 :11027, 2001.

[144] Y. Nakamura, Y.A. Pashkin, et J.S. Tsai. Coherent control of macroscopic quantum states in a single-Cooper-pair box. Nature (London), 398 :786, 1999.

[145] Y. Makhlin, G. Schön, et A. Shnirman. Josephson-junction qubits with controlled couplings. Nature (London), 398 :305, 1999.

[146] V. Bouchiat, D. Vion, P. Joyez, D. Esteve, et M.H. Devoret. Quantum coherence with a single Cooper pair. Physica Scripta, T76 :165, 1998.

[147] Y. Nakamura, Yu. A. Pashkin, T. Yamamoto, et J. S. Tsai. Rabi oscillations in a Josephson-junction charge two-level system. Phys. Rev. Lett., 87 :246601, 2001.

[148] Y. Nakamura, Yu. A. Pashkin, T. Yamamoto, et J. S. Tsai. Charge echo in a Cooper-pair box. Phys. Rev. Lett., 88 :047901, 2002.

[149] Yu. A. Pashkin, T. Yamamoto, O. Astafiev, Y. Nakamura, D.V. Averin, et J.S. Tsai. Quantum oscillations in two coupled charge qubits. cond-mat/0212314, 2002.

[150] D.V. Averin. Adiabatic quantum computation with cooper pairs. Solid State Commun., 105 :659, 1998.

[151] M.F. Bocko, A.M. Herr, et M.J. Feldman. Prospects for quantum coherent computation using superconducting electronics. IEEE Trans. Appl. Supercond., 7 :3638, 1997.

[152] W.K. Wootters et W.H. Zurek. A single quantum cannot be cloned. Nature, 299 :802, 1982.

[153] D. Dieks. Communication by EPR devices. Phys. Lett. A, 92 :271, 1982.

[154] R. Rouse, S. Han, et J. E. Lukens. Observation of resonant tunneling between macroscopically distinct quantum levels. Phys. Rev. Lett., 75 :1614, 1995.

[155] J.R. Friedman, V. Patel, W. Chen, S.K. Tolpygo, et J.E. Lukens. Detection of a Schroedinger's cat state in an rf-SQUID. Nature, $406: 43,2000$. 
[156] Caspar H. van der Wal, A. C. J. ter Haar, F. K. Wilhelm, R. N. Schouten, C. J. P. M. Harmans, T. P. Orlando, Seth Lloyd, et J. E. Mooij. Quantum superposition of macroscopic persistent-current states. Science, $290: 773,2000$.

[157] J.B. Majer, J.R. Butcher, et J.E. Mooij. Simple phase bias for superconducting circuits. Applied. Phys. Lett., 80 :3638, 2002.

[158] Z. Zhou, S.-I Chu, et S. Han. Quantum computing with superconducting devices : A three-level SQUID qubit. Phys. Rev. B, 66 :054527, 2002.

[159] J.I. Cirac et P. Zoller. Quantum computations with cold trapped ions. Phys. Rev. Lett., 74 :4091, 1995.

[160] A.M. Zagoskin. A scalable, tunable qubit, based on a clean DND or grain boundary D-D junction. cond-mat/9903170.

[161] G. Blatter, V.B. Geshkenbein, et L.V. Ioffe. Design aspects of superconducting phase quantum bits. Phys. Rev. B, 63 :174511, 2001.

[162] M.H.S. Amin, A.N. Omelyanchouk, A. Blais, Alec Maassen van den Brink, G. Rose, T. Duty, et A.M. Zagoskin. Multi-terminal superconducting phase qubit. Physica $C, 368: 310,2002$.

[163] A.M. Zagoskin. d-wave superconductors and quantum computers. Physica C, $368: 305,2002$.

[164] C. C. Tsuei et J. R. Kirtle. Pairing symmetry in cuprate superconductors. Rev. Mod. Phys., 72 :969, 2000.

[165] H. Hilgenkamp et J. Mannhart. Grain boundaries in high-Tc superconductors. Rev. Mod. Phys., 74 :485, 2002.

[166] E. Il'ichev, M. Grajcar, R. Hlubina, R. P. J. IJsselsteijn, H. E. Hoenig, H.-G. Meyer, A. Golubov, M. H. S. Amin, A. M. Zagoskin, A. N. Omelyanchouk, et M. Yu. Kupriyanov. Degenerate ground state in a mesoscopic $\mathrm{YBa} 2 \mathrm{Cu} 3 \mathrm{O} 7 \mathrm{x}$ grain boundary Josephson junction. Phys. Rev. Lett., 86 :5369, 2001.

[167] J.P. Heida, B.J. van Wees, T.M. Klapwijk, et G. Borghs. Nonlocal supercurrent in mesoscopic Josephson junctions. Phys. Rev. B, 57 :R5618, 1998.

[168] Gordon Baym. Lectures on Quantum Mechanics. Perseus Books, 1974. 
[169] R. Fazio, G. Massimo Palma, et J. Siewert. Fidelity and leakage of Josephson qubits. Phys. Rev. Lett., 83 :5385, 1999.

[170] F. Plastina, R. Fazio, et G.M. Palma. Macroscopic entanglement in Josephson nanocircuits. Phys. Rev. B, 64 :113306, 2001.

[171] P. Echternach, C.P. Williams, S.C. Dultz, S. L. Braunstein, et J. P. Dowling. Universal quantum gates for single cooper pair box based quantum computing. In R. Clark, editor, Proceedings of the 1st International Conference on Experimental Emplementations of Quantum Computation, page 111, Princeton, NJ, 2001. Rinton.

[172] D.Aharonov et M. Ben-Or. Polynomial simulations of decohered quantum computers. quant-ph/9611029, 1996.

[173] Y. Makhlin, G. Schön, et A. Shnirman. Nano-electronic realizations of quantum bits. J. Low Temp. Phys., $118: 751,2000$.

[174] A. Zagoskin et S. Charlebois. Communication personnelle.

[175] J.Q. You, J.S. Tsai, et F. Nori. Scalable quantum computing with josephson charge qubits. Phys. Rev. Lett., 89 :197902, 2002.

[176] J.Q. You, Chi-Hang Lam, et H.Z. Zheng. Superconducting charge qubits : The roles of self and mutual inductances. Phys. Rev. B, 73 :180501, 2001.

[177] M.H.S. Amin, A.N. Omelyanchouk, A. Blais, A. Maassen van den Brink, et A.M. Zagoskin. En préparation.

[178] J. Martinis. Communication personnelle.

[179] A. Blais, A. Maassen van den Brink, et A.M. Zagoskin. Tunable coupling of superconducting qubits. cond-mat/0207112.

[180] C. Cohen-Tannoudji, B. Diu, et F. Laloë. Mécanique Quantique, volume I et II. Hermann, Paris, 1973.

[181] J.M. Martinis, S. Nam, J. Aumentado, et C. Urbina. Decoherence of a superconducting qubit from bias noise. Non-publié.

[182] J.M. Raimond, M. Brune, et S. Haroche. Manipulating quantum entanglement with atoms and photons in a cavity. Rev. Mod. Phys., 73 :565, 2001. 
[183] D. Esteve. Communication personelle.

[184] N. Schuch et J. Siewert. A natural two-qubit gate for quantum computation using the xy interaction. quant-ph/0209035.

[185] D.A. Lidar et L.-A. Wu. Reducing constraints on quantum computer design by encoded selective recoupling. Phys. Rev. Lett., 88 :017905, 2002.

[186] L. Tian et S. Lloyd. Resonant cancellation of off-resonant effects in a multilevel qubit. Phys. Rev. A, $62: 050301(\mathrm{R}), 2000$.

[187] M.S. Bird L.-A. Wu et D.A. Lidar. Efficient universal leakage elimination for physical and encoded qubits. Phys. Rev. Lett., 89 :127901, 2002.

[188] F. Plastina et G. Falci. Communicating Josephson qubits. cond-mat/0206586.

[189] S. Haroche et J.M. Raimond. Manipulation of nonclassical field states in a cavity by atom interferometry. In P.R. Berman, editor, Cavity quantum electrodynamics, page 123. Academic Press, 1994.

[190] O. Buisson et F.W.J. Hekking. Entangled states in a Josephson charge qubit coupled to a superconducting resonator. cond-mat/0008275, 2001.

[191] F. Marquandt et C. Bruder. Superposition of two mesoscopically distinct quantum states : Coupling a Cooper-pair box to a large superconducting island. Phys. Rev. B, 63 :054514, 2001.

[192] W.A. Al-Saidi et D. Stroud. Eigenstates of a small Josephson junction coupled to a resonant cavity. Phys. Rev. B, $65: 014512,2001$.

[193] F.W.J. Hekking, O. Buisson, F. Balestro, et M.G. Vergniory. Cooper pair box coupled to a current-biased Josephson junction. cond-mat/0201284, 2002.

[194] P.R. Johnson, F.W. Strauch, A.J. Dragt, R.C. Ramos, C.J. Lobb, J.R. Anderson, et F.C. Wellstood. Spectroscopy of capacitively coupled josephson-junction qubits. cond-mat/0210278, 2002.

[195] S. Marchand, A. Blais, et A.-M. S. Tremblay. Coupling of charge quantum bit and current-biased josephson junction. Non publié, 2002.

[196] J.A. Wheeler et W.H. Zurek. Quantum theory and measurement. Princeton University Press, Princeton, 1983. 
[197] J. Bell. Against 'measurement'. Physics World, page 33, Août 1990.

[198] R. Peierls. In defence of 'measurement'. Physics World, page 19, Janvier 1991.

[199] J. Preskill. Quantum computing. Notes de cours, disponible sur le web http ://www.theory.caltech.edu/people/preskill/ph229/, 1997.

[200] G. Greenstein et A.G. Zajonc. The Quantum Challange. Jones and Barlett, London, 1997.

[201] J. von Neumann. Mathematical Foundations of Quantum Mechanics. Princeton University Press, 1955.

[202] A. N. Korotkov et D. V. Averin. Continuous weak measurement of quantum coherent oscillations. Phys. Rev. B, 64 :165310, 2001.

[203] D.V. Averin. Continuous weak measurement of the macroscopic quantum coherent oscillations. In Exploring the Quantum Classical Frontier : Recent Advances in Macroscopic Quantum Phenomena. Nova Science Publishers, 2002. Aussi disponible sur le web cond-mat/0004364.

[204] V.B. Braginsky et F.Y. Khalili. Quantum Measurement. Cambridge University Press, 1992.

[205] D.V. Averin. Quantum nondemolition measurement of a qubit. Phys. Rev. Lett., 88 :207901, 2002.

[206] M.H. Devoret et R.J. Schoelkopf. Amplifying quantum signals with the singleelectron transistor. Nature, $406: 1039,2000$.

[207] A.A. Clerk, S.M. Girvin, et A.D. Stone. Quantum-limited measurement and information in mesoscopic detectors. cond-mat/0211001, 2002.

[208] K.K. Likharev. Dynamics of Josephson jonctions and circuits. Gordon and Breach, New York, 1986.

[209] V.V. Danilov, K.K. Likharev, et O.V. Snirirev. Signal and noise parameters of SQUIDs. In SQUID '80, page 473, 1980.

[210] V.V. Danilov, K.K. Likharev, et A.B. Zorin. Quantum noise in SQUIDs. IEEE Trans. Mag., 19 :572, 1983.

[211] Y. Makhlin, G. Schön, et A. Shnirman. Josephson quantum bits in the flux regime. Physica C, $368: 276,2002$. 
[212] H. Tanaka, Y. Sekine, S. Saito, et H. Takayanagi. DC-SQUID readout for qubit. Physica C, 368 :300, 2002.

[213] E. Il'ichev, Th. Wagner, L. Fritzsch, J. Kunert, V. Schultze, T. May, H. E. Hoenig, H. G. Meyer, M. Grajcar, D. Born, W. Krech, M. V. Fistul, et A.M. Zagoksin. Characterization of superconducting structures designed for qubit realizations. Appl. Phys. Lett., 80 :4184, 2002.

[214] T.I. Robertson, B.L.T. Plourde, A. García-Martìnez, P.A. Reichardt, B. Chesca, R. Kleiner, Yu. Makhlin, G. Schön, A. Shnirman, F.K. Wilhelm, D.J. Van Harlingen, et J. Clarke. Superconducting device to isolate, entangle, and read-out quantum flux states. Non-publié, 2001.

[215] A. Shnirman et G. Schön. Quantum measurements performed with a singleelectron transistor. Phys. Rev. B, $57: 15400,1998$.

[216] A. Maassen van den Brink. Quantum-efficient charge detection using a singleelectron transistor. Euro. Phys. Lett., 58 :562, 2002.

[217] A.B. Zorin. Quantum-limited electrometer based on single Cooper pair tunneling. Phys. Rev. Lett., 76 :4408, 1996.

[218] A.B. Zorin. Ultimate sensitivity of the single Cooper pair tunneling electrometer. IEEE Trans. Ins. and Meas., 46 :299, 1997.

[219] A.B. Zorin. Radio-frequency Bloch-transistor electrometer. Phys. Rev. Lett., $86: 3388,2001$.

[220] A.B. Zorin. Cooper-pair qubit and Cooper-pair electrometer in one device. Physica C, 368 :284, 2002.

[221] A. Steinbach, P. Joyez, A. Cottet, D. Esteve, M. H. Devoret, M. E. Huber, et John M. Martinis. , Direct measurement of the josephson supercurrent in an ultrasmall Josephson junction. Phys. Rev. Lett., 87 :137003, 2001.

[222] M. Matters, W. J. Elion, et J. E. Mooij. Influence of controlled quantummechanical charge and phase fluctuations on Josephson tunneling. Phys. Rev. Lett., 75 :721, 1995.

[223] P. Joyez, P. Lafarge, A. Filipe, D. Esteve, et M. H. Devoret. Observation of parity-induced suppression of josephson tunneling in the superconducting single electron transistor. Phys. Rev. Lett., 1997 :2458, 1994. 
[224] A. Blais. Read-out device for phase qubits. Non-publié, Octobre 2001.

[225] A.N. Vystavkin, V.N. Gubankov, L.S. Kuzmin, K.K. Likharev, V.V. Mihulin, et V.K. Semenov. S-c-S junctions as nonlinear elements of microwave receiving devices. Rev. Phys. Appl., 9 :79, 1974.

[226] R.H. Koch, D.J. van Harligen, et J. Clarke. Quantum-noise theory for the resistively shunted Josephson junction. Phys. Rev. Lett., 45 :1232, 1980.

[227] R.H. Koch, D.J. van Harligen, et J. Clarke. Measurement of quantum noise in resistively shunted Josephson junctions. Phys. Rev. B, 26 :74, 1982.

[228] M. Watanabe et David B. Haviland. Coulomb blockade and coherent singlecooper-pair tunneling in single Josephson junctions. Phys. Rev. Lett., 86 :5120, 2001.

[229] V. Schöllmann, P. Agren, D. B. Haviland, T. H. Hansson, et A. Karlhede. Sample and hold strategy for quantum measurements of Josephson charge qubits. Phys. Rev. B, 65 :020505(R), 2002. 